ADVANCED STEEL CONSTRUCTION

An International Journal

Volume 16 Number 3

September 2020

CONTENTS

Technical Papers

An Improved Explicit-Implicit Precise Integration Method for Nonlinear Dynamic Analysis of Structures Zhi-Xia Ding, Zuo-Lei Du, Wei Su and Yao-Peng Liu

An Experimental Study on Mechanical Properties of Concrete-Filled Steel Tube (CFST) Key Connected Prefabricatec Wall and Column

Qian Wu, Guo-Chang Li, Ming Li and Yuan-Wei Luo

Experimental and Theoretical Study on the Behavior of the Laminated Action of Steel-Concrete Composite Beam in Negative Bending Moment Region

Xian Liang, Shu-Jin Duan, Yuan-Yuan Wang and Yan-Oing Zhang

Seismic Behavior of Biaxial Hollow Reinforced Concrete Slab to Concrete Filled Steel Tubular Column Connections

Chao Gong, Zhao-Xin Hou, Guo-Zhong Cheng, Y. Frank Chen and Wei-Qiao Liang

Experimental Study and Theoretical Analysis on Seismic Performance of Castellated Beam with Hexagonal Holes

Lian-Guang Jia, Ran Bi, Yu-Xiao Lang and Xue-Feng Li

An Efficient Method for Yield and Failure Surfaces of the Steel I-section

Anh Q Vu, Nghia H Hoang and Hien M Nghiem

Experimental Study of Resilient Prefabricated Steel Frame with All-Bolted Beam-To-Column Connections

Ai-Lin Zhang, Guang-Hao Shangguan, Yan-Xia Zhang, Qing-Bo Wang and Wen-Chao Cai

Influence of Different Shapes of Geometric Imperfections on the Structural Behavior of Beams with Large Web Openings Teixeira F. B., Caldas R. B. and Grilo L. F.

Analysis of Beam-To-Upright End Connections Steel Storage Systems

Lucas Alves Escanio, Guilherme Cássio Elias, Luiz Henrique de Almeida Neiva, Vinícius Nicchio Alves

and Arlene Maria Cunha Sarmanho

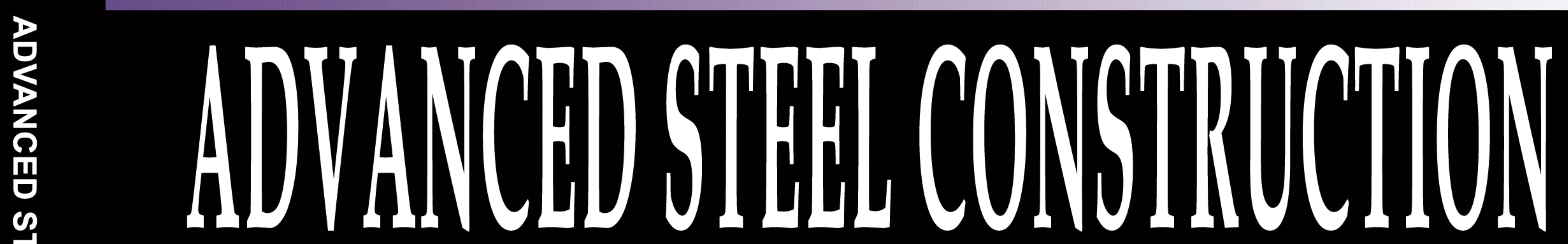

an International Joumal ISSN 1816-112X

Volume 16 Number 3

September 2020

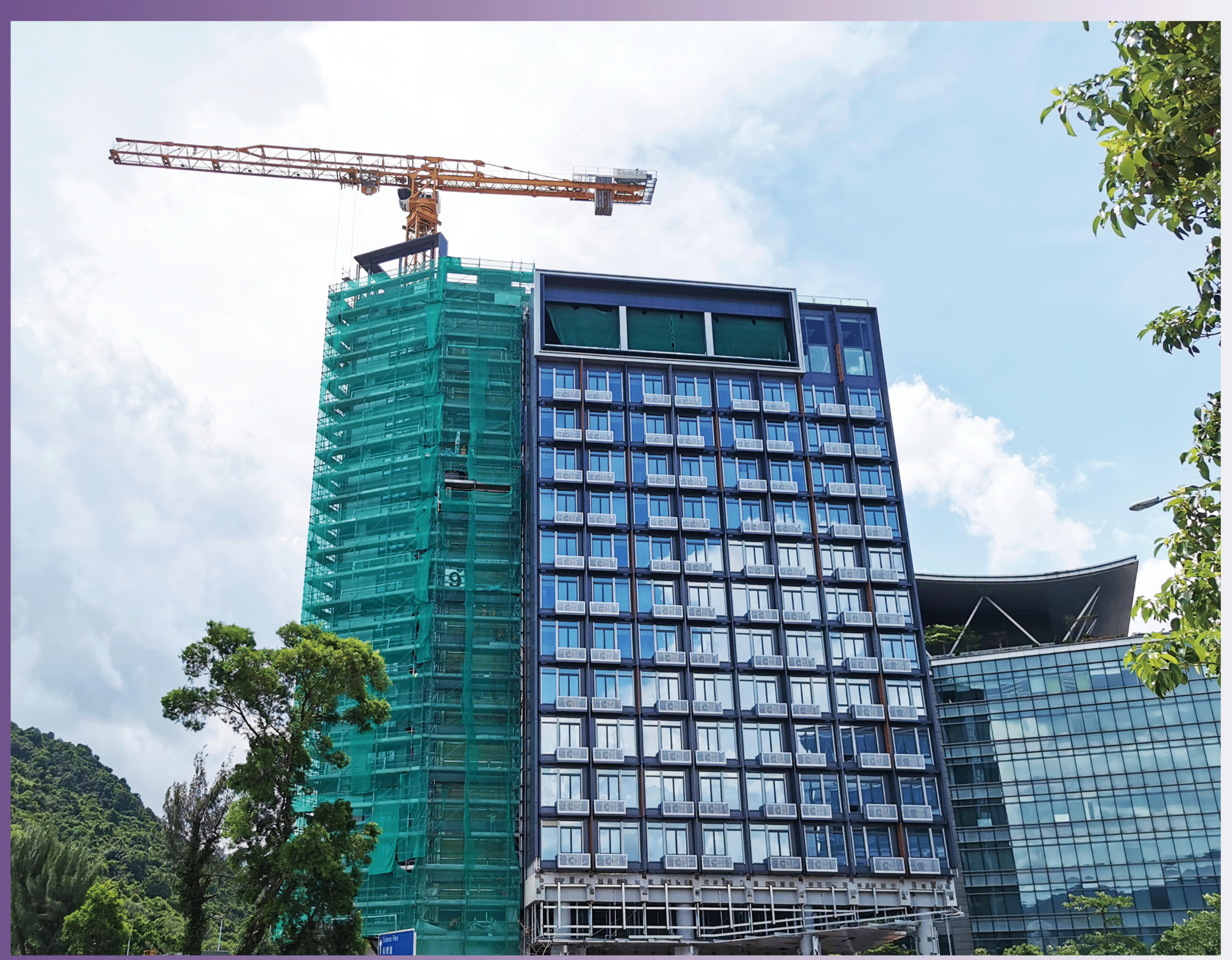

Editors-in-Chief

S.L. Chan, The Hong Kong Polytechnic University, Hong Kong, China

W.F. Chen, University of Hawaii at Manoa, USA

R. Zandonini, Trento University, Italy 


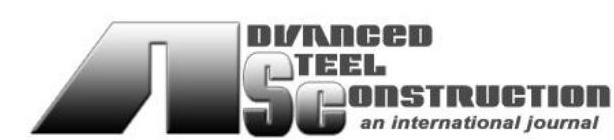

ISSN 1816-112X

Science Citation Index Expanded, Materials Science Citation Index and ISI Alerting

\begin{tabular}{|c|c|}
\hline EDITORS-IN-CHIEF & L.H. Han \\
\hline $\begin{array}{l}\text { Asian Pacific, African } \\
\text { and organizing Editor } \\
\text { S.L. Chan } \\
\text { The Hong Kong Polyt. Univ., } \\
\text { Hong Kong, China }\end{array}$ & $\begin{array}{l}\text { Tsinghua Univ. China } \\
\text { S. Herion } \\
\text { University of Karlsruhe, Germany }\end{array}$ \\
\hline $\begin{array}{l}\text { American Editor } \\
\text { W.F. Chen } \\
\text { Univ. of Hawaii at Manoa, USA }\end{array}$ & $\begin{array}{l}\text { Ove Arup \& Partners Hong Kong Ltd., Hong Kong, } \\
\text { China }\end{array}$ \\
\hline $\begin{array}{l}\text { European Editor } \\
\text { R. Zandonini } \\
\text { Trento Univ., Italy }\end{array}$ & $\begin{array}{l}\text { B.A. Izzuddin } \\
\text { Imperial College of Science, Technology and } \\
\text { Medicine, UK }\end{array}$ \\
\hline ASSOCIATE EDITORS & J.P. Jaspart \\
\hline $\begin{array}{l}\text { Y.P. Liu } \\
\text { The Hong Kong Polyt. Univ., Hong Kong, China }\end{array}$ & $\begin{array}{l}\text { Univ. of Liege, Belgium } \\
\text { S. A. Jayachandran }\end{array}$ \\
\hline $\begin{array}{l}\text { S.W. Liu } \\
\text { Sun Yat-Sen Univ., China }\end{array}$ & $\begin{array}{l}\text { IIT Madras, Chennai, India } \\
\text { S.E. Kim }\end{array}$ \\
\hline $\begin{array}{l}\text { INTERNATIONAL } \\
\text { EDITORIAL BOARD }\end{array}$ & Sejong Univ., South Korea \\
\hline $\begin{array}{l}\text { F.G. Albermani } \\
\text { Central Queensland Univ., Australia }\end{array}$ & $\begin{array}{l}\text { S. Kitipornchai } \\
\text { The Univ., of Queensland, Australia }\end{array}$ \\
\hline $\begin{array}{l}\text { I. Burgess } \\
\text { Univ. of Sheffield, UK }\end{array}$ & $\begin{array}{l}\text { D. Lam } \\
\text { Univ. of Bradford, UK }\end{array}$ \\
\hline $\begin{array}{l}\text { F.S.K. Bijlaard } \\
\text { Delft Univ. of Technology, The Netherlands }\end{array}$ & $\begin{array}{l}\text { H.F. Lam } \\
\text { City Univ. of Hong Kong, Hong Kong, China }\end{array}$ \\
\hline $\begin{array}{l}\text { R. Bjorhovde } \\
\text { The Bjorhovde Group, USA }\end{array}$ & $\begin{array}{l}\text { G.Q. Li } \\
\text { Tongji Univ., China }\end{array}$ \\
\hline $\begin{array}{l}\text { M.A. Bradford } \\
\text { The Univ. of New South Wales, Australia }\end{array}$ & $\begin{array}{l}\text { J.Y.R. Liew } \\
\text { National Univ. of Singapore, Singapore }\end{array}$ \\
\hline $\begin{array}{l}\text { D. Camotim } \\
\text { Technical Univ. of Lisbon, Portugal }\end{array}$ & $\begin{array}{l}\text { E.M. Lui } \\
\text { Syracuse Univ., USA }\end{array}$ \\
\hline $\begin{array}{l}\text { C.M. Chan } \\
\text { Hong Kong Univ. of Science \& Technology, Hong Kong, China }\end{array}$ & $\begin{array}{l}\text { Y.L. Mo } \\
\text { Univ. of Houston, USA }\end{array}$ \\
\hline $\begin{array}{l}\text { T.H.T. Chan } \\
\text { Queensland Univ. of Technology, Australia }\end{array}$ & $\begin{array}{l}\text { J.P. Muzeau } \\
\text { CUST, Clermont Ferrand, France }\end{array}$ \\
\hline $\begin{array}{l}\text { T.M. Chan } \\
\text { The Hong Kong Polyt. Univ., Hong Kong, China }\end{array}$ & $\begin{array}{l}\text { D.A. Nethercot } \\
\text { Imperial College of Science, Technology and } \\
\text { Medicine, UK }\end{array}$ \\
\hline $\begin{array}{l}\text { Z.H. Chen } \\
\text { Tianjin Univ., China }\end{array}$ & $\begin{array}{l}\text { Y.Q. Ni } \\
\text { The Hong Kong Polyt. Univ., Hong Kong, China }\end{array}$ \\
\hline $\begin{array}{l}\text { S.P. Chiew } \\
\text { Nanyang Technological Univ., Singapore }\end{array}$ & $\begin{array}{l}\text { D.J. Oehlers } \\
\text { The Univ. of Adelaide, Australia }\end{array}$ \\
\hline $\begin{array}{l}\text { W.K. Chow } \\
\text { The Hong Kong Polyt. Univ., Hong Kong, China }\end{array}$ & $\begin{array}{l}\text { J.L. Peng } \\
\text { Yunlin Uni. of Science \& Technology, Taiwan, China }\end{array}$ \\
\hline $\begin{array}{l}\text { G.G. Deierlein } \\
\text { Stanford Univ., California, USA }\end{array}$ & $\begin{array}{l}\text { K. Rasmussen } \\
\text { The Univ. of Sydney, Australia }\end{array}$ \\
\hline $\begin{array}{l}\text { L. Dezi } \\
\text { Univ. of Ancona, Italy }\end{array}$ & $\begin{array}{l}\text { J.M. Rotter } \\
\text { The Univ. of Edinburgh, UK }\end{array}$ \\
\hline $\begin{array}{l}\text { D. Dubina } \\
\text { The Politehnica Univ. of Timosoara, Romania }\end{array}$ & $\begin{array}{l}\text { C. Scawthorn } \\
\text { Scawthorn Porter Associates, USA }\end{array}$ \\
\hline $\begin{array}{l}\text { R. Greiner } \\
\text { Technical Univ. of Graz, Austria }\end{array}$ & $\begin{array}{l}\text { P. Schaumann } \\
\text { Univ. of Hannover, Germany }\end{array}$ \\
\hline $\begin{array}{l}\text { L. Gardner } \\
\text { Imperial College of Science, Technology and Medicine, UK }\end{array}$ & $\begin{array}{l}\text { Y.J. Shi } \\
\text { Tsinghua Univ., China }\end{array}$ \\
\hline $\begin{array}{l}\text { Y. Goto } \\
\text { Nagoya Institute of Technology, Japan }\end{array}$ & $\begin{array}{l}\text { G.P. Shu } \\
\text { Southeast Univ. China }\end{array}$ \\
\hline
\end{tabular}

\section{Advanced Steel Construction an international journal}

\author{
L. Simões da Silva \\ Department of Civil Engineering, University of \\ Coimbra, Portugal \\ J.G. Teng \\ The Hong Kong Polyt. Univ., Hong Kong, China \\ G.S. Tong \\ Zhejiang Univ., China \\ K.C. Tsai \\ National Taiwan Univ., Taiwan, China \\ C.M. Uang \\ Univ. of California, USA \\ B. Uy \\ University of Western Sydney, Australia \\ M. Veljkovic \\ Univ. of Lulea, Sweden \\ F. Wald \\ Czech Technical Univ. in Prague, Czech \\ Y.C. Wang \\ The Univ. of Manchester, UK \\ Y.L. Xu \\ The Hong Kong Polyt. Univ., Hong Kong, China \\ D. White \\ Georgia Institute of Technology, USA \\ E. Yamaguchi \\ Kyushu Institute of Technology, Japan \\ Y.B. Yang \\ National Taiwan Univ., Taiwan, China \\ Y.Y. Yang \\ China Academy of Building Research, Beijing, China \\ B. Young \\ The Univ. of Hong Kong, Hong Kong, China \\ X.L. Zhao \\ Monash Univ., Australia \\ X.H. Zhou \\ Chongqing University, China \\ Z.H. Zhou \\ The Hong Kong Polyt. Univ., Hong Kong, China \\ S.Y. Zhu \\ The Hong Kong Polyt. Univ., Hong Kong, China \\ R.D. Ziemian \\ Bucknell Univ., USA
}




\section{General Information Advanced Steel Construction, an international journal}

\section{Aims and scope}

The International Journal of Advanced Steel Construction provides a platform for the publication and rapid dissemination of original and up-to-date research and technological developments in steel construction, design and analysis. Scope of research papers published in this journal includes but is not limited to theoretical and experimental research on elements, assemblages, systems, material, design philosophy and codification, standards, fabrication, projects of innovative nature and computer techniques. The journal is specifically tailored to channel the exchange of technological know-how between researchers and practitioners. Contributions from all aspects related to the recent developments of advanced steel construction are welcome. 


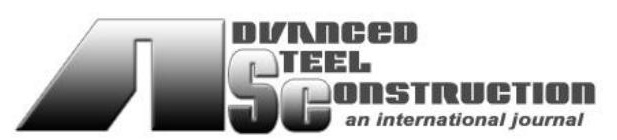

ISSN 1816-112X

Science Citation Index Expanded, Materials Science Citation Index and ISI Alerting

\section{EDITORS-IN-CHIEF}

\section{Asian Pacific, African and organizing Editor}

S.L. Chan

The Hong Kong Polyt. Univ., Hong Kong, China

Email: ceslchan@polyu.edu.hk

\section{American Editor}

W.F. Chen

Univ. of Hawaii at Manoa, USA

Email:waifah@hawaii.edu

\section{European Editor}

R. Zandonini

Trento Univ., Italy

Email: riccardo.zandonini@ing.unitn.it

\section{Advanced Steel Construction an international journal}

\section{VOLUME 16 NUMBER 3}

September 2020

Technical Papers

An Improved Explicit-Implicit Precise Integration Method for Nonlinear Dynamic 19 Analysis of Structures

Zhi-Xia Ding, Zuo-Lei Du, Wei Su* and Yao-Peng Liu

An Experimental Study on Mechanical Properties of Concrete-Filled Steel Tube 206 (CFST) Key Connected Prefabricated Wall and Column

Qian Wu, Guo-Chang Li*, Ming Li and Yuan-Wei Luo

Experimental and Theoretical Study on the Behavior of the Laminated Action of 216 Steel-Concrete Composite Beam in Negative Bending Moment Region

Xian Liang, Shu-Jin Duan*, Yuan-Yuan Wang and Yan-Qing Zhang

Seismic Behavior of Biaxial Hollow Reinforced Concrete Slab to Concrete Filled 223 Steel Tubular Column Connections

Chao Gong, Zhao-Xin Hou, Guo-Zhong Cheng*, Y. Frank Chen and Wei-Qiao Liang

Experimental Study and Theoretical Analysis on Seismic Performance of 233 Castellated Beam with Hexagonal Holes

Lian-Guang Jia*, Ran Bi, Yu-Xiao Lang and Xue-Feng Li

An Efficient Method for Yield and Failure Surfaces of the Steel I-section Anh $Q V u^{*}$, Nghia H Hoang, and Hien M Nghiem

Experimental Study of Resilient Prefabricated Steel Frame with All-Bolted 255 Beam-To-Column Connections

Ai-Lin Zhang, Guang-Hao Shangguan, Yan-Xia Zhang*, Qing-Bo Wang and Wen-Chao Cai

Influence of Different Shapes of Geometric Imperfections on the Structural 272 Behavior of Beams with Large Web Openings

Teixeira F. B. *, Caldas R. B. and Grilo L. F.

Analysis of Beam-To-Upright End Connections Steel Storage Systems 279 Lucas Alves Escanio, Guilherme Cássio Elias, Luiz Henrique de Almeida Neiva, Vinícius Nicchio Alves and Arlene Maria Cunha Sarmanho* 



\title{
AN IMPROVED EXPLICIT-IMPLICIT PRECISE INTEGRATION METHOD FOR NONLINEAR DYNAMIC ANALYSIS OF STRUCTURES
}

\author{
Zhi-Xia Ding ${ }^{1}$, Zuo-Lei Du ${ }^{1}$, Wei Su ${ }^{2, *}$ and Yao-Peng Liu ${ }^{1,3}$ \\ ${ }^{1}$ Department of Civil and Environmental Engineering, The Hong Kong Polytechnic University, Hung Hom, Kowloon, Hong Kong, China \\ ${ }^{2}$ School of Aeronautics and Astronautics, Sun Yat-sen University, Guangzhou, 510275, China \\ ${ }^{3}$ Nida Technology Co. Ltd., Hong Kong Science Park, Shatin, N.T., Hong Kong, China \\ *(Corresponding author: E-mail: suwei@mail.sysu.edu.cn)
}

\section{A B S T RA C T}

In this paper, an improved explicit-implicit precise integration method (EIPIM) is proposed for nonlinear dynamic analysis of structures based on the refined precise integration method (RPIM). The RPIM may face a numerical instability problem when using large time steps while the proposed EIPIM uses an explicit predictor-implicit corrector scheme to provide more stable and accurate results and allow for large time steps. When using EIPIM, the unknown displacements at the starting point are predicted by an explicit function, and the trial displacements at the second step are corrected by the implicit Lagrange interpolation method. In terms of numerical stability and precision, eight explicit formulas have been evaluated to develop a better predictor for EIPIM, i.e., two third-order, four fourth-order and two fifth-order functions. Four examples involving both linear and nonlinear problems illustrate the high stability and numerical efficiency of the proposed method. It is found that the explicit predictors EIPIM_O31 and EIPIM_O41 show good performance and are recommended for nonlinear dynamic analysis.
A R T I C LE H IST TORY

$\begin{array}{ll}\text { Received: } & \text { 11 May } 2019 \\ \text { Revised: } & \text { 19 November } 2019 \\ \text { Accepted: } & \text { 15 December } 2019\end{array}$

\section{K E Y W O R D S}

Precise integration method; Nonlinear dynamic analysis; Explicit predictor-implicit corrector scheme;

Stability and accuracy

\section{Introduction}

Wind-induced response [1] and wave-induced response [2] are the two important applications of dynamic analysis in the design process of offshore steel structures. The precise integration method (PIM) with high accuracy and explicit recursion was firstly proposed by Zhong and Williams [3-4] for structural dynamic analysis. In the past decsades, many researchers made significant contributions on the development and improvement of PIM. Nowadays, it has been applied to many fields, including optimal control, heat conduction, wave propagation, partial differential equation, etc. [5]. The concept of PIM is to convert the second-order ordinary differential equation (ODE) to a first-order ODE by introducing a vector, through which more accurate results can be obtained with high numerical stability. Nevertheless, the size of matrices is doubled when using PIM, leading to increased computational efforts. Many efforts have been made to reduce computational efforts [6-8] and handle nonlinear inhomogeneous parts [9-11]. However, very few studies focused on incorporating PIM into the nonlinear structural dynamic analysis. Zhang et al. [12] incorporated PIM into dynamic elastic-plastic hardening and softening problems in which only a motion equation with constant stiffness matrices is involved. The authors of this paper proposed the refined precise integration method (RPIM) [13], introducing a new vector representing velocity that is an improvement over Zhong [3-4, 14] and Gao [15]. In this method, the operation on doubled matrices is simplified to the operation on $2 \times 2$ matrices. The stiffness matrix is treated as an unknown variable to adapt RPIM into nonlinear structural problems. However, similar to other explicit and implicit methods [16], the stability problem may also occur when a large time increment is adopted even though RPIM is unconditionally stable with a spectral radius of less than 1 .

Both implicit and explicit methods are employed in commercial or uncommercial packages for structural analysis, for example, ABAQUS [17] and OpenSees [18]. The explicit procedures, such as the central difference method (CDM), are conditionally stable and demand smaller time increments to achieve accurate results. Implicit procedures, such as the Newmark method [19], trapezoidal rule [20], and Wilson $\theta$-method [21], are unconditionally stable in the linear analysis but can become unstable in the nonlinear analysis [16]. Though explicit methods avoid the procedure of iteration, having been explored by many researchers[22-23], truncation error during the procedure will be accumulated and lead to inaccurate results or difficulty in convergence. For implicit methods, the error will be eliminated through several steps of iteration, and the accuracy of results is guaranteed within pre-defined tolerance. Therefore, the implicit methods can employ larger time increments. The composite implicit time integration method is a procedure with two sub-steps per time step and the two times computational effort for a certain time step size is compensated unconditionally by the achieved stability. Bathe $[16,24]$ extended the trapezoidal rule by combing with the three-point Euler backward method in the second sub-step, and it is verified to be unconditional stability. This method was also proved to be applicable for large time step sizes in both linear and nonlinear structural analysis of very flexible and stiff problems [25-26]. Zhang et al. [27] studied the accuracy of a generalized composite implicit method combining the Newmark method and three-point backward Euler method, and then an accuracy analysis framework was recommended for composite schemes. Kim and Choi [28] improved an implicit composite time integration algorithm with the weighted residual method for linear analysis, and the weighting parameters are optimized with a single degree of freedom (DOF) problem. In these studies, the results with second order accuracy as well as unconditional stability was achieved.

To incorporate the excellent stability of implicit methods into the explicit method, many researchers developed the adaptive explicit method, in which an adaptive time stepping procedure is adopted to control time discretization error in each step [29]. One of the most popular adaptive strategies is the local error estimator incorporated in each step. The adaptive Rung-Kutta method [30-31] provides an estimation of the local truncation error of a single Rung-Kutta step; thus, the error can be controlled within a limit by changing to a smaller step, which can be automatically selected. The automatic time step control strategy by Hulbert and Jang [32], based on the derived local error estimator was demonstrated with reliable step size behavior. Deokar et al. [33] proposed an independent posteriori error estimator based on the generalized single step single solve (GSSSS) framework and adaptive time steps saved much computational effort. Wang and Zhong [34] employed a priori error analysis in the proposed time finite element method for structural dynamics. Mayr et al. [35] developed an adaptive time stepping scheme for fluid-structure interaction (FSI) problems with a posteriori error estimation by comparing two different schemes. Meanwhile, other adaptive strategies on numerical time integration for dynamic structural analysis, such as the algorithm of Bergan and Mollestad based on current characteristic frequency, and the method of Lages et al. based on the geometric indicator of displacements history curvature, have been studied by researchers [36]. Although the adaptive strategies have advantages in terms of accuracy and efficiency, they are difficult to incorporate into RPIM because their coefficients have to be recalculated for a different time increment. Nevertheless, the idea of estimation in each step could be combined with RPIM to improve stability [37].

Inspired by composite implicit methods and adaptive time stepping procedures, an explicit-implicit precise integration method (EIPIM) is proposed in this paper. We propose RPIM to deal with nonlinear dynamic problems based on PIM by Zhong [3] and an additional theorem of Duhamel integration by Tan [38]. The unconditional spectral stability and high accuracy of RPIM have been verified. To possess the advantages of both explicit and implicit methods, explicit functions are employed in EIPIM to estimate 
displacements for the next step while the implicit Lagrange interpolation method is adopted in the recursion formula. Eight estimation functions with three different orders of accuracy are proposed and evaluated in this paper, both theoretically and practically. We employed four cases involving beam element, truss element, and shell element to test and verify the stability and efficiency of EIPIMs with different estimation functions for both linear and nonlinear dynamic analysis of structures.

\section{Conventional central difference method}

In dynamic structural problems, the equation of motion is defined as

$$
\mathbf{M} \ddot{x}+\mathbf{C} \dot{x}+\mathbf{K} \boldsymbol{x}=\mathbf{f}(\boldsymbol{x}, \mathrm{t})
$$

in which, $\mathrm{M}, \mathrm{C}$, and $\mathrm{K}$ are the mass, damping, and stiffness matrices of the structural system respectively; $\mathbf{f}(\boldsymbol{x}, \mathrm{t})$ is the external force vector related to time and displacements. $\boldsymbol{x}, \dot{\boldsymbol{x}}$, and $\ddot{\boldsymbol{x}}$ are the displacement, velocity, and acceleration vectors of each DOF.

The velocity and acceleration are written as

$$
\begin{aligned}
& \dot{\boldsymbol{x}}_{t+\Delta t / 2}=\left(\boldsymbol{x}_{t+\Delta t}-\boldsymbol{x}_{t}\right) / \Delta t \\
& \ddot{\boldsymbol{x}}_{t}=\left(\dot{\boldsymbol{x}}_{t+\Delta t / 2}-\dot{\boldsymbol{x}}_{t-\Delta t / 2}\right) / \Delta t
\end{aligned}
$$

where $\boldsymbol{x}$ is a DOF (e.g., displacement or rotation), the subscript t refers to the current time step and $\Delta t$ refers to the time increment in the dynamic analysis.

The CDM is conditionally stable with the stability limit given in terms of the system or the elements as

$$
\begin{gathered}
\Delta t \leq \frac{2}{\omega_{\max }} \\
\Delta t \leq \frac{2}{\omega_{\max }^{\text {element }}}
\end{gathered}
$$

in which $\omega_{\max }$ is the highest eigenvalue of the system and $\omega_{\max }^{\text {element }}$ is the maximum eigenvalue of elements.

The estimation for $\Delta t$ is only approximate and in most cases is not a conservative estimation [17]. In the nonlinear analysis, the stable time limit is going to change with the highest frequency of model; and thus, the time increment has to be estimated at every step. Otherwise, a small enough time increment should be used to ensure stable solutions. An approximation to the stable time limit can also be written as the smallest transit time of a dilatational wave across any of the elements in the mesh, which clearly demonstrates the relationship between stable time limit and element size.

\section{RPIM for dynamic structural problems}

To deal with nonlinear dynamic analysis, the authors proposed the RPIM based on the traditional PIM [3] and the additional theorem of Duhamel integration derived by Tan [39]. A new vector $p$, representing velocity is employed, and the matrix for coefficients calculation is converted to a $2 \times 2$ matrix, significantly simplifying the computation and saving storage space.

\subsection{Refined precise integration method (RPIM)}

The authors proposed RPIM to deal with nonlinear dynamic problems in which the stiffness matrix $\mathrm{K}$ varies with time in elastic-plastic range. The third item, $\mathbf{K} \boldsymbol{x}$ in equation of motion Eq.(1) is considered as an unknown variable, and a new vector $\mathbf{F}(\boldsymbol{x}, t)$ combining the external force vector $\mathbf{f}(\boldsymbol{x}, t)$ and internal force $\mathrm{Kx}$ is formed. Then, the equation of motion can be rewritten as

$$
\mathbf{M} \ddot{\boldsymbol{x}}+\mathbf{C} \dot{\boldsymbol{x}}=\mathbf{f}(\boldsymbol{x}, t)-\mathbf{K} \boldsymbol{x}=\mathbf{F}(\boldsymbol{x}, t)
$$

A new vector $\mathrm{p}$, representing the velocity, is introduced as

$$
p=\dot{x}
$$

Writing Eq.(6) and Eq.(7) in matrix form,

$$
\boldsymbol{u}=\left[\begin{array}{l}
x \\
\dot{x}
\end{array}\right], \quad \boldsymbol{u}=\mathbf{H} \dot{u}+\mathbf{F}
$$

in which

$$
\begin{aligned}
& \mathbf{H}=\left[\begin{array}{cc}
\mathbf{0} & \mathbf{I} \\
\mathbf{0} & -\mathbf{M}^{-1} \mathbf{C}
\end{array}\right] \\
& \mathbf{F}=\left[\begin{array}{c}
\mathbf{0} \\
\mathbf{F}(\boldsymbol{x}, t)
\end{array}\right]=\left[\begin{array}{c}
\mathbf{0} \\
\mathbf{M}^{-1} \mathbf{f}(\boldsymbol{x}, t)-\mathbf{M}^{-1} \mathbf{K} \boldsymbol{x}
\end{array}\right]
\end{aligned}
$$

The absolute solution of Eq.(8) with Duhamel integral is given mathematically as Eq.(11).

$$
\boldsymbol{u}(\mathrm{t})=\exp (\mathbf{H} t) \boldsymbol{x}(0)+\int_{0}^{t} \exp (\mathbf{H}(t-\tau)) \mathbf{F}(x, \tau) d \tau
$$

The total time is divided into a series of step time $\eta$ for numerical integration, and such that the time points are given as,

$$
t_{0}=0, t_{1}=\eta, t_{2}=2 \eta, \ldots, t_{k}=k \eta, t_{k+1}=(k+1) \eta, \ldots
$$

The integration equation of Eq.(11) can be given as Eq.(13) with the mark $\mathrm{uk}$ representing $\mathrm{u}(\mathrm{k})$ and $t_{k}$ representing $t(k)$.

$$
\boldsymbol{u}_{k+1}=\boldsymbol{\Phi} \boldsymbol{u}_{k}+\int_{0}^{\eta} \exp \left(\mathbf{H}\left(t_{k}-\tau\right)\right) \mathbf{F}\left(\boldsymbol{x}_{k}, \tau\right) d \tau
$$

In which the matrix exponent is defined as Eq.(14), and the additional theorem is proposed by Zhong [3] to give an accurate solution.

$$
\boldsymbol{\Phi}=\exp (\mathbf{H} \eta)
$$

For more easy integration, the formed 'external force' $\mathbf{F}(\boldsymbol{x}, t)$ is written in polynomial form as,

$$
\mathbf{F}\left(\boldsymbol{x}_{k}, t_{k}+\tau\right)=\boldsymbol{f}_{0, k}+\tau \boldsymbol{f}_{1, k}+\cdots+\tau^{m} \boldsymbol{f}_{m, k} \quad \tau \in[0, \eta]
$$

The recursion formula can then be derived as Eq.(16) by substituting Eq.(15) into the general solution in Eq.(13).

$$
\boldsymbol{v}_{k+1}=\boldsymbol{\Phi} \boldsymbol{v}_{k}+\eta \boldsymbol{\Phi}_{0} \boldsymbol{f}_{0, k}+\cdots+\eta^{m+1} \boldsymbol{\Phi}_{m} \boldsymbol{f}_{m, k}
$$

in which,

$$
\boldsymbol{\Phi}_{m}=\frac{1}{\eta^{m+1}} \int_{0}^{\eta} \exp (\mathbf{H}(\eta-\tau)) \tau^{m} d \tau
$$

The additional theorem of Duhamel integration by Tan [38] can then be employed to achieve precise coefficients $\boldsymbol{\Phi}_{m}$ with the combination of the exponential matrix by Zhong [3].

With the coefficient $\beta$ in Rayleigh damping [40] ignored, the damping matrix $\mathrm{C}$ is only proportional to the mass matrix $\mathbf{M}$ and then the $\mathbf{H}$ matrix is simplified as a $2 \times 2$ matrix. Thus, the coefficient matrix $\boldsymbol{\Phi}$ becomes a matrix with two different constant coefficients described as $\boldsymbol{\Phi}^{\prime}$, and only constant elements exist in coefficients $\boldsymbol{\Phi}_{0}, \boldsymbol{\Phi}_{1}, \boldsymbol{\Phi}_{2}$, and $\boldsymbol{\Phi}_{3}$, as well as the linear operation results $\overline{\boldsymbol{\Phi}}_{0}, \overline{\boldsymbol{\Phi}}_{1}, \overline{\boldsymbol{\Phi}}_{2}$, and $\overline{\boldsymbol{\Phi}}_{3}$.

$$
\boldsymbol{\Phi}^{\prime}=\left[\begin{array}{ll}
1 & c_{1} \\
0 & c_{2}
\end{array}\right]
$$

$$
\boldsymbol{\Phi}_{0}=\left[\begin{array}{ll}
\Phi_{01} \\
\Phi_{02}
\end{array}\right], \boldsymbol{\Phi}_{1}=\left[\begin{array}{ll}
\Phi_{11} \\
& \Phi_{12}
\end{array}\right], \boldsymbol{\Phi}_{2}=\left[\begin{array}{ll}
\Phi_{21} \\
\Phi_{22}
\end{array}\right], \boldsymbol{\Phi}_{3}
$$




$$
\begin{aligned}
\overline{\boldsymbol{\Phi}}_{0}=\left[\begin{array}{ll}
\bar{\Phi}_{01} \\
\bar{\Phi}_{02}
\end{array}\right], \overline{\boldsymbol{\Phi}}_{1} & =\left[\begin{array}{ll}
\bar{\Phi}_{11} \\
\bar{\Phi}_{12}
\end{array}\right], \overline{\boldsymbol{\Phi}}_{2}=\left[\begin{array}{ll}
\bar{\Phi}_{21} \\
\bar{\Phi}_{22}
\end{array}\right], \overline{\boldsymbol{\Phi}}_{3} \\
& =\left[\begin{array}{ll}
\bar{\Phi}_{31} \\
\bar{\Phi}_{32}
\end{array}\right]
\end{aligned}
$$

Thus, the multiplication between matrices and vectors becomes simple multiplication between constant values and vectors, and a large amount of computation time and memory size are saved; this is applicable to both consistent and lumped mass matrix for structures

\subsection{Implicit method for inhomogeneous term}

Explicit method is adopted with Lagrange interpolation in the RPIM; however, inaccuracy and instability may occur with large time increment due to the integration in Eq.(13) over the time domain [tk, tk+1]. To alleviate this problem, an implicit method with three Lagrange integration points is adopted in the EIPIM.

The Lagrange polynomials 'external force' $\mathbf{F}(\boldsymbol{x}, t)$ within the time period $\left[t_{k}, t_{k+1}\right]$ with integration points $t_{k+1}, t_{k}, t_{k-1}, t_{k-2}$ derived given as,

$$
\begin{aligned}
& \tilde{\mathbf{F}}\left(\boldsymbol{x}_{k}, t_{k}+\tau\right) \\
& =\boldsymbol{f}_{k+1}+\frac{\boldsymbol{f}_{k+1} / 3+\boldsymbol{f}_{k} / 2-\boldsymbol{f}_{k-1}+\boldsymbol{f}_{k-2} / 6}{\eta} \tau \\
& +\frac{\boldsymbol{f}_{k+1} / 2-\boldsymbol{f}_{k}+\boldsymbol{f}_{k-1} / 2}{\eta^{2}} \tau^{2} \\
& +\frac{\boldsymbol{f}_{k+1} / 6-\boldsymbol{f}_{k} / 2+\boldsymbol{f}_{k-1} / 2-\boldsymbol{f}_{k-2} / 6}{\eta^{3}} \tau^{3}
\end{aligned}
$$

Comparing Eq.(20) with Eq.(15), the recursion formula is then established as,

$$
\boldsymbol{u}_{k+1}=\boldsymbol{\Phi} \boldsymbol{u}_{k}+\eta\left(\overline{\boldsymbol{\Phi}}_{0} \boldsymbol{f}_{k+1}+\overline{\boldsymbol{\Phi}}_{1} \boldsymbol{f}_{k}+\overline{\boldsymbol{\Phi}}_{2} \boldsymbol{f}_{k-1}+\overline{\boldsymbol{\Phi}}_{3} \boldsymbol{f}_{k-2}\right)
$$

in which

$$
\begin{aligned}
& \overline{\boldsymbol{\Phi}}_{0}=\boldsymbol{\Phi}_{1} / 3+\boldsymbol{\Phi}_{2} / 2+\boldsymbol{\Phi}_{3} / 6 \\
& \overline{\boldsymbol{\Phi}}_{1}=\boldsymbol{\Phi}_{0}+\boldsymbol{\Phi}_{1} / 2-\boldsymbol{\Phi}_{2}-\boldsymbol{\Phi}_{3} / 2 \\
& \overline{\boldsymbol{\Phi}}_{2}=-\boldsymbol{\Phi}_{1}+\boldsymbol{\Phi}_{2} / 2+\boldsymbol{\Phi}_{3} / 2 \\
& \overline{\boldsymbol{\Phi}}_{3}=\boldsymbol{\Phi}_{1} / 6-\boldsymbol{\Phi}_{3} / 6
\end{aligned}
$$

The external forces are known and linearly distributed during each time increment in structural engineering, such that the external force can be written as a linear function in the time domain $\left[t_{k}, t_{k+1}\right]$ as Eq.(23) with an exact result provided by Duhamel integration.

$$
\mathbf{f}\left(t_{k}+\tau\right)=\mathbf{f}_{k}+\tau\left(\mathbf{f}_{k+1}-\mathbf{f}_{k}\right) / \eta
$$

\subsection{Estimation with the explicit method}

The explicit method with the three-point forward Lagrange interpolation function may result in inaccurate values (Fig. 1(a)) or even opposite trends (Fig. 1(b)) when large time increments are adopted for the estimation of $\boldsymbol{x}_{k+1}$ in the next time increment. As illustrated in Fig. 1, exact values of functions, $\sin (\pi \mathrm{t})$ and $\sin 3(\pi \mathrm{t})$ respectively, are given in red stars. The functions of the four-point forward Lagrange interpolation method over the time domain within four-time increments are shown with colored lines. Time is divided into 30 equal time increments within $[-0.5,1]$ for each function and $t_{k}$ is adopted as $\mathrm{t}=0.5$. 'Lagrange1' represents interpolating with points at $t_{k-3}, t_{k-2}, t_{k-1}$ and $t_{k}$, which are the dotted point with red stars. Similarly, 'Largrange2' standards for interpolating with every two points, the points at $t_{k-6}, t_{k-4}, t_{k-2}$ and $t_{k}$, etc.

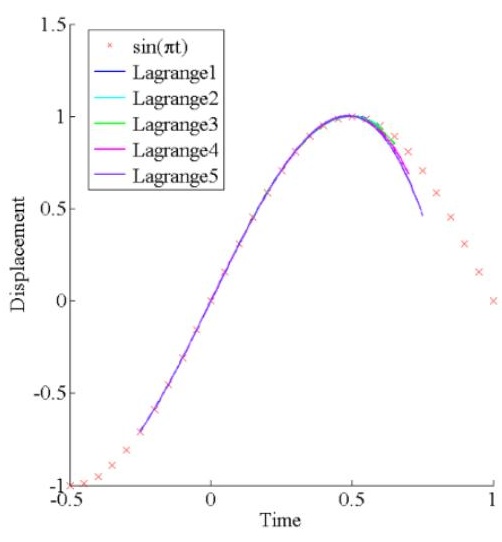

(a) Fitting of $\sin (\pi \mathrm{t})$

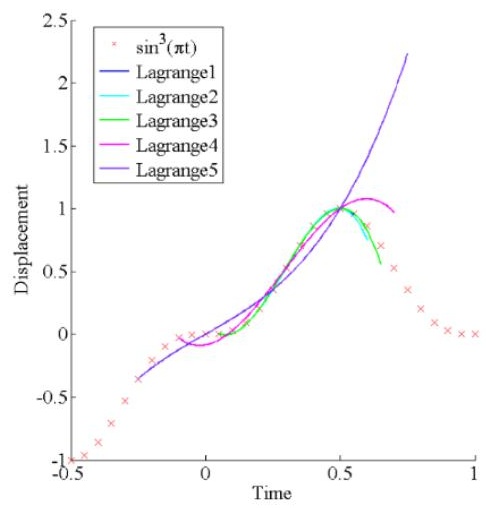

(b) Fitting of $\sin 3(\pi \mathrm{t})$

Fig. 1 Accuracy of the forward Lagrange interpolation with different time increments

As clearly seen in Fig. 1(a), the estimated value for $\sin (\pi \mathrm{t} k+1)$ (value for the next time step at time $t k+1$ for Lagrane1, $t k+2$ for Lagrane2, etc.) is lower

\begin{tabular}{|c|c|c|}
\hline \multirow{2}{*}{$\begin{array}{l}\text { Third- } \\
\text { order } \\
\qquad \boldsymbol{O}\left(\eta^{3}\right)\end{array}$} & $\boldsymbol{x}_{k+1, \text { estimate }}=\boldsymbol{x}_{k-1}+2 \eta \boldsymbol{v}_{k}$ & $(24)$ \\
\hline & $\boldsymbol{x}_{k+1, \text { estimate }}=3 \boldsymbol{x}_{k}-3 \boldsymbol{x}_{k-1}+\boldsymbol{x}_{k-2}$ & $(25)$ \\
\hline \multirow{4}{*}{$\begin{array}{l}\text { Fourth- } \\
\text { order } \\
\qquad \boldsymbol{O}\left(\eta^{4}\right)\end{array}$} & $\boldsymbol{x}_{k+1, \text { estimate }}=2 \boldsymbol{x}_{k}-\boldsymbol{x}_{k-1}+\eta^{2} \boldsymbol{a}_{k}$ & (26) \\
\hline & $\boldsymbol{x}_{k+1, \mathrm{estimate}}=4 \boldsymbol{x}_{k}-6 \boldsymbol{x}_{k-1}+4 x_{k-2}-\boldsymbol{x}_{k-3}$ & $(27)$ \\
\hline & $\boldsymbol{x}_{k+1, \mathrm{estimate}}=-4 \boldsymbol{x}_{k}+5 \boldsymbol{x}_{k-1}+4 \eta \boldsymbol{v}_{k}+2 \eta \boldsymbol{v}_{k-1}$ & $(28)$ \\
\hline & $\boldsymbol{x}_{k+1, \text { estimate }}=-3 \boldsymbol{x}_{k} / 2+3 \boldsymbol{x}_{k-1}-\boldsymbol{x}_{k-2} / 2+3 \eta \boldsymbol{v}_{k}$ & $(29)$ \\
\hline \multirow{3}{*}{$\begin{array}{l}\text { Fifth-order } \\
\qquad \boldsymbol{O}\left(\eta^{5}\right)\end{array}$} & $x_{k+1, \text { estimate }}=-9 x_{k}+9 x_{k-1}+x_{k-2}+6 \eta v_{k}$ & (30) \\
\hline & $++6 \eta v_{k-1}$ & \\
\hline & $\begin{aligned} \boldsymbol{x}_{k+1, \text { estimate }}=15 \boldsymbol{x}_{k} / 4- & 3 \boldsymbol{x}_{k-1}+\boldsymbol{x}_{k-2} / 4-3 \eta \boldsymbol{v}_{k} / 2 \\
+ & 3 \eta^{2} \boldsymbol{a}_{k} / 2\end{aligned}$ & $(31)$ \\
\hline
\end{tabular}
than the exact result, and larger discrepancies are observed in larger time increments even with the right variational trend. Nevertheless, the variational trend of Lagrange interpolation is opposite with large time increments (e.g., Largange4 and Largange5) in Fig. 1(b). The deviation accumulates during the integration with the Duhamel method as well as the recursion formulation in Eq.(13). Thus, estimation with better methods is required to minimize this error.

Totally eight methods with three different orders of accuracy are introduced by adopting the Lagrange interpolation method and the Hermite interpolation method. Due to the ill-condition of high order interpolation function, functions with higher order (e.g., sixth-order and above) are not introduced or considered in this paper.

The explicit-implicit method with each estimation function from Eq.(24) to Eq.(31) is defined as EIPIM_O31, EIPIM_O32, EIPIM_O41, EIPIM_O42, EIPIM_O43, EIPIM_O44, EIPIM_O51, and EIPIM_O52, respectively, indicating the order of accuracy. The EIPIMs stand for a total of eight methods with different estimation functions. 


\section{The algorithm of EIPIM}

For nonlinear analysis with stiffness matrix $\mathrm{K}$ depending on displacements of structures, the recursion formula of EIPIM is given in Eq.(32) based on the RPIM, before which, the estimation functions in Eq.(24) to Eq.(31) should be employed for $\boldsymbol{x}_{k+1, \text { estimate }}$ and $\mathbf{K}_{k+1 \text {,estimate }}$ is evaluated with $\boldsymbol{x}_{k+1 \text {,estimate }}$ thereafter. In linear analysis, $\mathbf{K}_{k+1, \text { estimate }}$ should be replaced by constant $\mathrm{K}$.

$$
\begin{aligned}
& \boldsymbol{x}_{k+1}=\boldsymbol{x}_{k}+\boldsymbol{T}_{12} \boldsymbol{v}_{k}+ \eta \mathbf{M}^{-1}\left[\Phi_{01} \boldsymbol{f}_{k}+\Phi_{11}\left(\boldsymbol{f}_{k+1}-\boldsymbol{f}_{k}\right)\right] \\
&+\eta \mathbf{M}^{-1}\left(\bar{\Phi}_{01} \mathbf{K}_{k+1, \text { estimate }} \boldsymbol{x}_{k+1, \text { estimate }}\right. \\
&+\bar{\Phi}_{11} \mathbf{K}_{k} \boldsymbol{x}_{k}+\bar{\Phi}_{21} \mathbf{K}_{k-1} \boldsymbol{x}_{k-1} \\
&\left.+\bar{\Phi}_{31} \mathbf{K}_{k-2} \boldsymbol{x}_{k-2}\right) \\
& \boldsymbol{v}_{k+1}=\boldsymbol{T}_{22} \boldsymbol{v}_{k}+\eta \mathbf{M}^{-1}\left[\Phi_{02} \boldsymbol{f}_{k}+\Phi_{12}\left(\boldsymbol{f}_{k+1}-\boldsymbol{f}_{k}\right)\right] \\
&+\eta \mathbf{M}^{-1}\left(\bar{\Phi}_{02} \mathbf{K}_{k+1, \text { estimate }} \boldsymbol{x}_{k+1, \text { estimate }}\right. \\
&+\bar{\Phi}_{12} \mathbf{K}_{k} \boldsymbol{x}_{k}+\bar{\Phi}_{22} \mathbf{K}_{k-1} \boldsymbol{x}_{k-1} \\
&\left.+\bar{\Phi}_{32} \mathbf{K}_{k-2} \boldsymbol{x}_{k-2}\right)
\end{aligned}
$$

The difference of algorithms between the EIPIM and RPIM is that the displacements and stiffness matrix should be estimated before the calculation of displacements and velocities for the next time increment. In this paper, the CDM is adopted to calculate the required results at the first two or three steps for both linear and nonlinear problems, as illustrated in Fig. 2. The first two steps are needed in the EIPIM_O31, EIPIM_O32, EIPIM_O41, and EIPIM_O43 because there is no requirement either on displacement at time tk-1 or velocity at time tk-2. Acceleration should be updated if involved in estimation formulae, such as the EIPIM_O41 and EIPIM_O52.

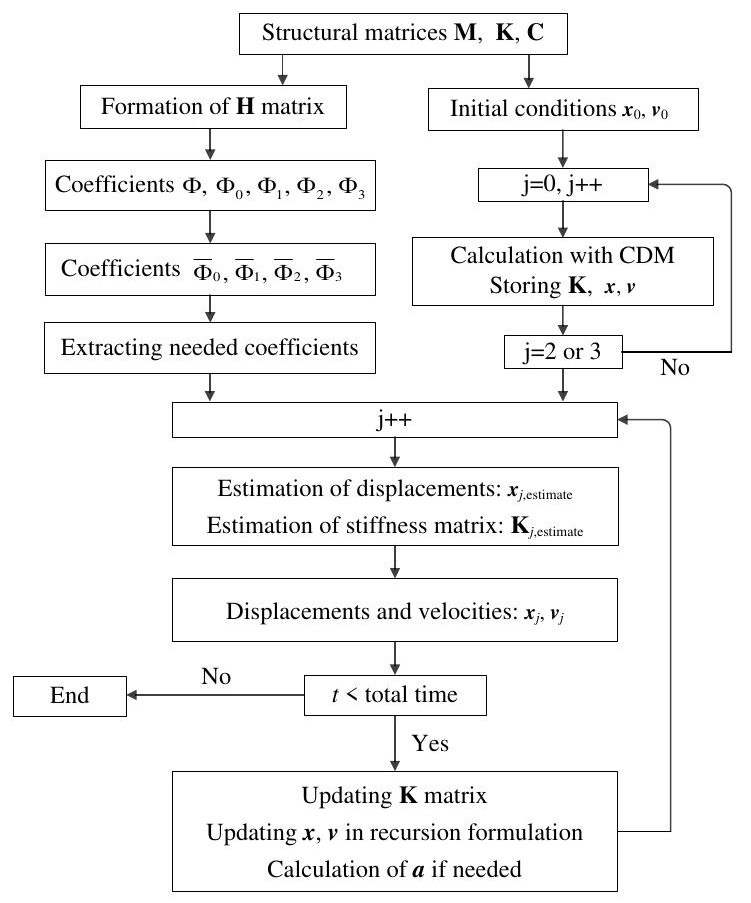

Fig. 2 Algorithm flowchart of EIPIM

\section{Stability and precision analysis}

In this section, we analyze the two most important characteristics of an algorithm [41], stability and precision, and compare the performance of EIPIM with RPIM.

\subsection{Stability analysis}

The recursion formula is unconditionally stable if the solution will not magnify without limits at any step time. The authors verified the stability of RPIM, and unconditional spectral stability in theory with the spectral radius of $\boldsymbol{\Phi}$ smaller than 1 in another paper. Nevertheless, the approximation in Duhamel integration over time tk (current time) and tk+1 (time at next step) in Eq.(13) may lead to instability due to the unknown function of internal force, multiplication of stiffness matrix $\mathrm{K}$, and displacements $\mathrm{x}$. In the RPIM, the explicit Lagrange interpolation method with four previous $\mathrm{K}$ and $\mathrm{x}$ is adopted in recursion formula. As illustrated in Fig. 1, unsuitable functions, with large time increments for the next step, will produce wrong tendencies. The implicit Lagrange interpolation method is employed in the EIPIM in Eq.(20) and better stability will be provided if the estimation of displacement at time tk+1 is accurate enough.

For the eight EIPIMs, estimation formulas with third-order, forth-order and fifth-order accuracy involving displacements, velocities, and accelerations may have different effects on the stability of recursion formula. Among these methods, EIPIM_O32 and EIPIM_O42 only adopting displacements in estimation function, which is the Lagrange interpolation method mathematically, means that only displacements are satisfied at the discretized time points. Methods with velocity and/or acceleration involved in estimation function (Hermit interpolation function, in essence), satisfy not only the displacement but also the velocity/acceleration at certain time points. The EIPIM_O41 achieves the fourth-order accuracy with only three parameters, reducing the accumulation of initial magnitude error-error resulting from inaccurate initial parameters used to make an estimation. Thus, the EIPIM_O41 exhibits the highest stability theoretically. The EIPIM_O51 and EIPIM_O52 may become unstable with relatively smaller time increments due to the illcondition of interpolation functions with higher order accuracy.

\subsection{Precision analysis}

For both the EIPIM and RPIM, integration of inhomogeneous terms Kx is the only approximation during the derivation of recursion formula for equation of motion. The RPIM with the fourth order explicit Lagrange interpolation function has a precision of $O\left(\Delta t^{5}\right)$ which is closely related to the time increment $\Delta t$.

With a similar derivation process, the recursion formulation of Eq.(8) can be written as,

$\boldsymbol{u}_{k+1}=\boldsymbol{\Phi} \boldsymbol{u}_{k}+\eta\left(\overline{\boldsymbol{\Phi}}_{0} \mathbf{F}_{k+1}+\overline{\boldsymbol{\Phi}}_{1} \mathbf{F}_{k}+\overline{\boldsymbol{\Phi}}_{2} \mathbf{F}_{k-1}+\overline{\boldsymbol{\Phi}}_{3} \mathbf{F}_{k-2}\right)$

Combining the addition theorem and the coefficients in Eq.(22), and expanding the $\mathbf{F}_{k+1}, \mathbf{F}_{k-1}$ and $\mathbf{F}_{k-2}$ at $\mathbf{F}_{k}$ with Taylor series, then Eq.(33) can be derived as,

$$
\begin{aligned}
\boldsymbol{u}_{k+1}=\boldsymbol{\Phi} \boldsymbol{u}_{k}+\eta \overline{\boldsymbol{\Phi}}_{0}( & \left.\boldsymbol{u}_{k}^{(1)}-\boldsymbol{u}_{k}\right) \\
& +\sum_{j=0}^{\infty}\left(\frac{(\eta)^{j}}{j !} \overline{\boldsymbol{\Phi}}_{1}+\frac{(-\eta)^{j}}{j !} \overline{\boldsymbol{\Phi}}_{2}\right. \\
& \left.+\frac{(-2 \eta)^{j}}{j !} \overline{\boldsymbol{\Phi}}_{3}\right)\left(\boldsymbol{u}_{k}^{(j+1)}-\boldsymbol{u}_{k}^{(j)}\right) \\
& =\boldsymbol{u}_{k}+\eta \boldsymbol{u}_{k}^{(1)}+\frac{\eta^{2}}{2} \boldsymbol{u}_{k}^{(2)}+\frac{\eta^{3}}{6} \boldsymbol{u}_{k}^{(3)} \\
& +\frac{\eta^{4}}{24} \boldsymbol{u}_{k}^{(4)} \\
& +\frac{\eta^{5}}{24}\left(\frac{5}{6} \boldsymbol{u}_{k}^{(5)}+\frac{31}{30} \mathbf{H} \boldsymbol{u}_{k}^{(4)}+O(\eta)\right)+\cdots
\end{aligned}
$$

Thus, the EIPIM with implicit Lagrange interpolation function has the precision of $O\left(\Delta t^{5}\right)$, the same accuracy as the RPIM in theory.

\section{Verification examples}

Several examples are analyzed to assess the stability and efficiency of the proposed EIPIMs in dealing with linear and nonlinear dynamic analysis. The free vibration and forced vibration with constant and varied external forces of a cantilever are studied to provide better understanding and easier duplication of the EIPIMs, as well as a demonstration in a linear analysis. Besides the proposed EIPIMs with eight different estimation functions, results from the other three different methods, namely ODE45(MATLAB), CDM, and RPIM, are given and compared with EIPIMs in linear dynamic analysis. The results from function ode45 [42] in MATLAB are used as references, in which the fourth-order and fifth-order Runge-Kutta algorithm are adopted, commonly used for nonstiff problems. In nonlinear dynamic analysis, results from MATLAB is not available because of its disability in nonlinear analysis. 


\subsection{Free vibration of a linear elastic cantilever}

A linear elastic cantilever with initial displacement as shown in Fig. 3 is employed to verify the accuracy and stability of the EIPIMs. The beam is divided into ten beam-column elements with equal length. Three DOFs are considered for each node, namely axial displacement, vertical displacement, and rotation. The element mass matrix $\mathrm{m}$ and element stiffness matrix $\mathrm{k}$ are given as follows, with elastic modulus $\mathrm{E}=2.0 \times 1010 \mathrm{~N} / \mathrm{m} 2$, the length of element $1=0.4 \mathrm{~m}$ (total length of beam $\mathrm{L}=4 \mathrm{~m}$ ), section area $\mathrm{A}=0.1 \mathrm{~m} 2$, second moment of inertia $\mathrm{I}=1 / 480 \mathrm{~m} 4$. The damping matrix is proportional to the mass matrix with the relationship $\mathrm{C}=0.2 \mathrm{M}$. Elemental matrices are assembled to the global matrix according to DOFs of each node. The cantilever with initial displacements is illustrated in Fig. 3 with dashed lines. The maximum displacement at the free tip is $1 \mathrm{~m}$, and the initial displacements vary with the cubic of distances from the clamped edge at each node. The initial velocity is a zero vector, indicating no initial velocity.

$$
\begin{aligned}
& \mathbf{m}=\frac{\rho A l}{2}\left[\begin{array}{llllll}
1 & & & & & \\
& 1 & & & & \\
& & 1 & & & \\
& & & 1 & & \\
& & & & 1 & \\
& & & & & 1
\end{array}\right] \\
& \mathbf{k}=\left[\begin{array}{cccccc}
\frac{E A}{l} & 0 & 0 & -\frac{E A}{l} & 0 & 0 \\
& \frac{12 E I}{l^{3}} & \frac{6 E I}{l^{2}} & 0 & -\frac{12 E I}{l^{3}} & \frac{6 E I}{l^{2}} \\
& & \frac{4 E I}{l} & 0 & -\frac{6 E I}{l^{2}} & \frac{2 E I}{l} \\
& & & \frac{E A}{l} & 0 & 0 \\
& m & & \frac{12 E I}{l^{3}} & -\frac{6 E I}{l^{2}} \\
& & y & & & \frac{4 E I}{l}
\end{array}\right]
\end{aligned}
$$

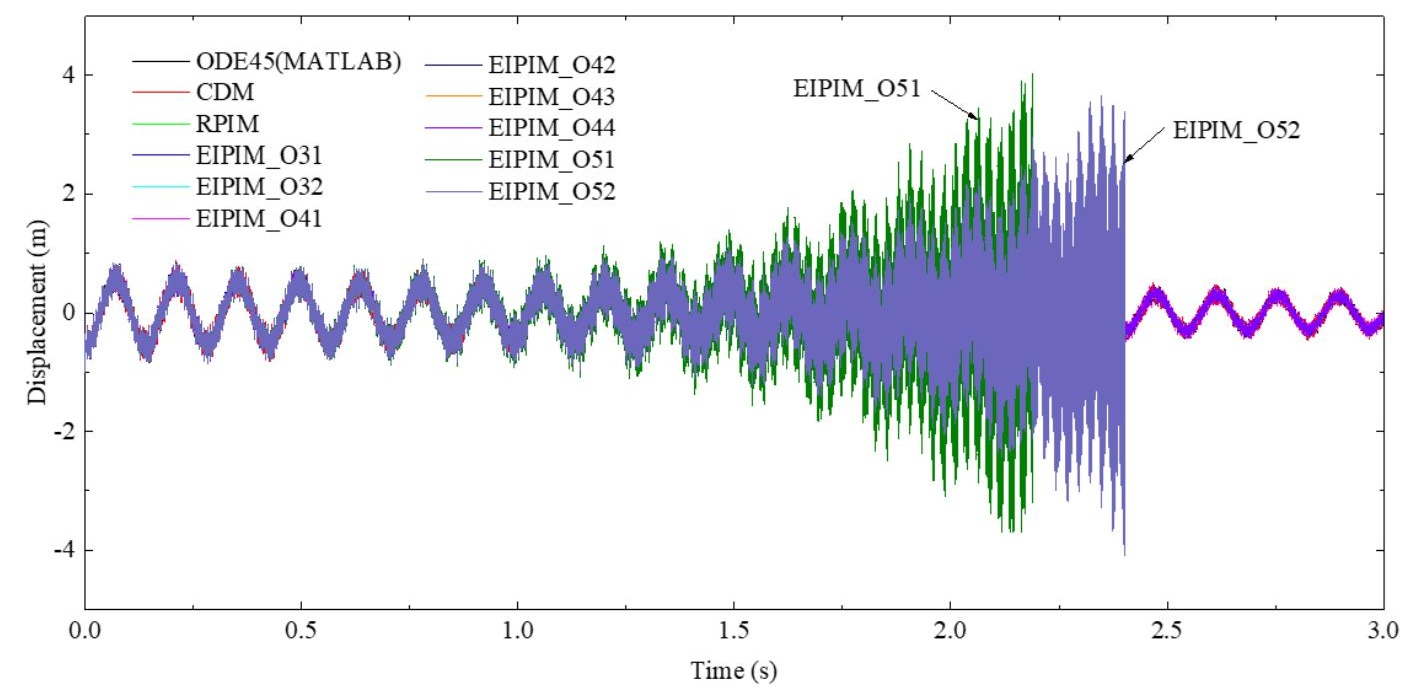

(a) Time-displacement curve with time increment $\Delta \mathrm{t}=0.00004 \mathrm{~s}$ applicable in section 6.2.
Typical time-displacement curves with certain time increments are illustrated in Fig. 4. For time increment $\Delta t=0.00004 \mathrm{~s}$, results from EIPIM with the fifth order-accuracy, EIPIM_O51, and EIPIM_O52 exhibit instability as shown in Fig. 4(a), while other methods can still give reasonable predictions of displacements. This is primarily due to the ill-condition of higher order interpolation, as discussed in section 3.3. When time increment increases to $\Delta t$ $=0.00011 \mathrm{~s}$ as shown in Fig. 4(b), the RPIM starts to become unstable at the very beginning at about 0.003s, and EIPIM_O32, EIPIM_O42, EIPIM_O51, and EIPIM_O52 all suffer instability in only 0.03s. As shown in Fig. 4(b), results from the CDM vary quite a lot from the exact results of MATLAB, while other methods (EIPIM_O31, EIPIM_O41, EIPIM_O43, and EIPIM_O44) provide estimations much closer to exact solutions.

It should be noted that the results from the EIPIM_O42 are the same as those from the RPIM. This is due to the constant stiffness matrix and the same estimation method, fourth-order Lagrange interpolation method. To be specific, the combination of explicit fourth-order Lagrange interpolation method for estimation and implicit fourth-order Lagrange interpolation method to calculate displacement at the next step in the EIPIM_O42 results in the same function during the time domain, which is also the same as explicit Lagrange interpolation method in the recursion formula of the RPIM. Due to the constant stiffness matrix in linear elastic problems, the same coefficient is multiplied to displacements, and thus EIPIM_O42 and RPIM yield to the same solutions resulting from the same function for displacements, which will also be

As shown in Fig. 4(c), for the time increment $\Delta t=0.00016$ s, only the EIPIM_O31 and EIPIM_O41 can provide reasonable results being close to those from MATLAB. When the time increment increases, the EIPIM can no longer give stable result. Nevertheless, the stable time limit $\Delta \mathrm{t}=0.00016 \mathrm{~s}$ of the EIPIM_O31 and EIPIM_O41 is more than 1.5 times larger than $\Delta \mathrm{t}=0.0001 \mathrm{~s}$ of RPIM, indicating superior stability of the EIPIM_O31 and EIPIM_O41. 


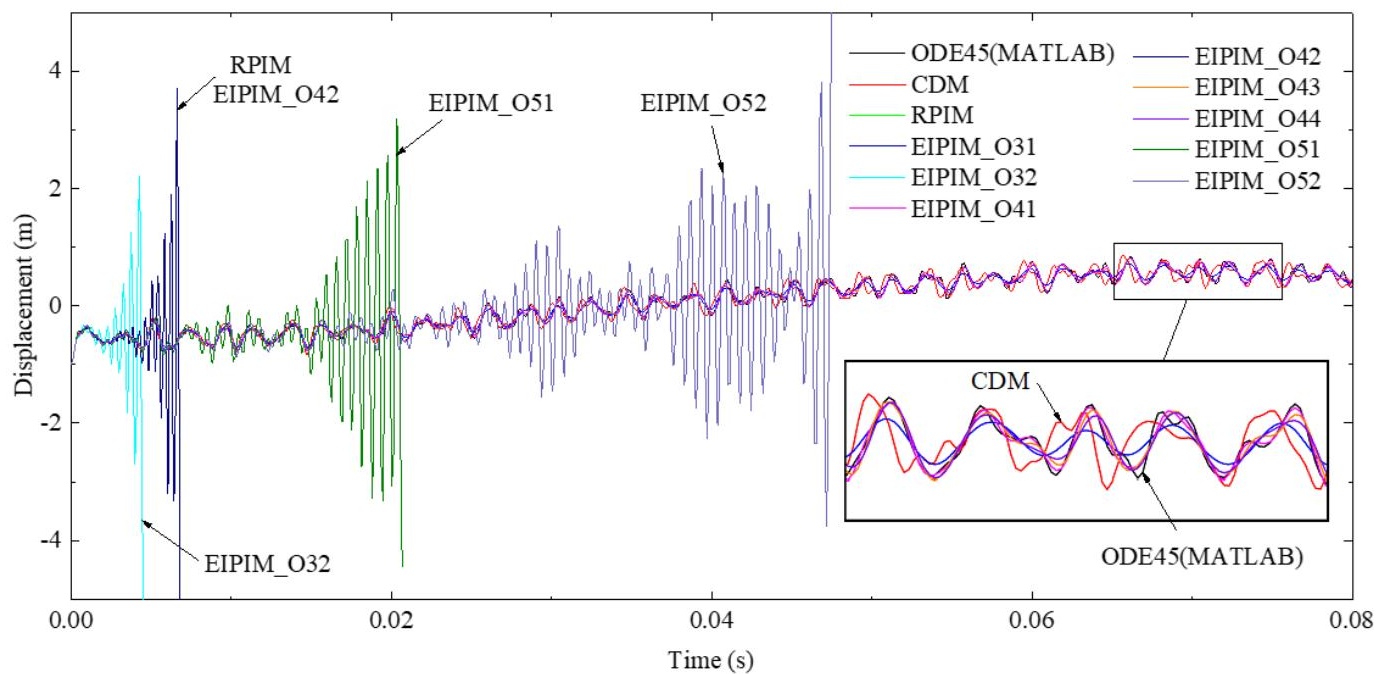

(b) Time-displacement curve with time increment $\Delta \mathrm{t}=0.00011 \mathrm{~s}$

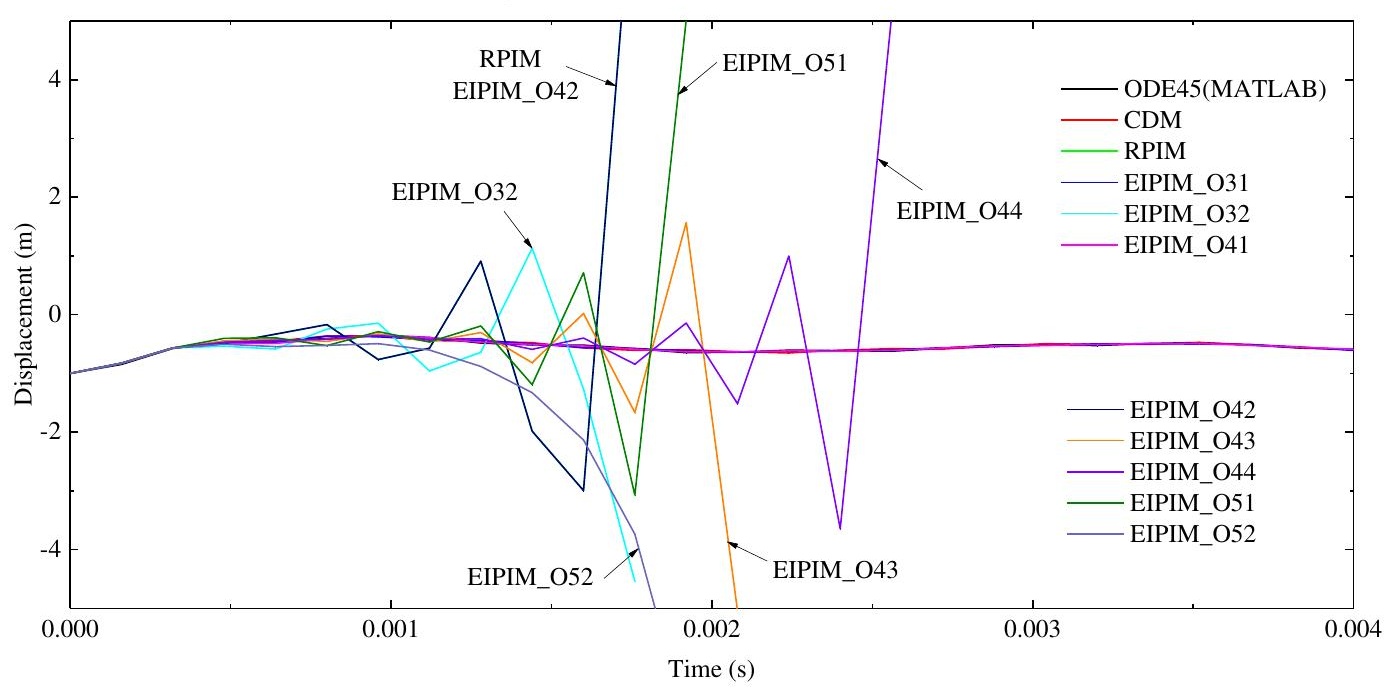

(c) Time-displacement curve with time increment $\Delta \mathrm{t}=0.00016 \mathrm{~s}$

Fig. 4 Time-displacement curves of the cantilever beam with free vibration

\subsection{Forced vibration of a linear elastic cantilever}

In this example, a cantilever beam, with the same section and material properties and the same boundary condition as in section 6.1 , is employed. The differences lie in both null vectors of initial displacement and velocity, and the constant vertical external force on free tip $F=100 \mathrm{kN}$. Similarly, only the displacements at the free tip are monitored for different time increments as illustrated in Fig. 5.

At time increment $\Delta \mathrm{t}=0.00007 \mathrm{~s}$, the EIPIMs with fifth order accuracy of estimation, EIPIM_O51, and EIPIM_O52, start to be unstable within 0.5s, while stable and reasonable results are obtained from other methods, as shown in Fig. 5(a). The RPIM cannot work well from $\Delta \mathrm{t}=0.00011 \mathrm{~s}$ and the same results are provided with those from EIPIM_O42, as discussed in section 6.1. The EIPIM_O32, EIPIM_51, and EIPIM_O52 could not remain effective at this time increment, as shown in Fig. 5(b). Similar to free variation case, the RPIM and all EIPIMs, except EIPIM_O31 and EIPIM_O41, yield to instability at $\Delta \mathrm{t}$ $=0.00016 \mathrm{~s}$, while EIPIM_O31 and EIPIM_O4 1 provide accurate results very close to those from MATLAB, as illustrated in Fig. 5(c) and (d). Comparing with the results from different methods in section 6.1 with free vibration, the stability of different methods are nearly the same except for small differences in EIPIM_O44, EIPIM_O51, and EIPIM_O52, which will be further compared and discussed at the end of this section.

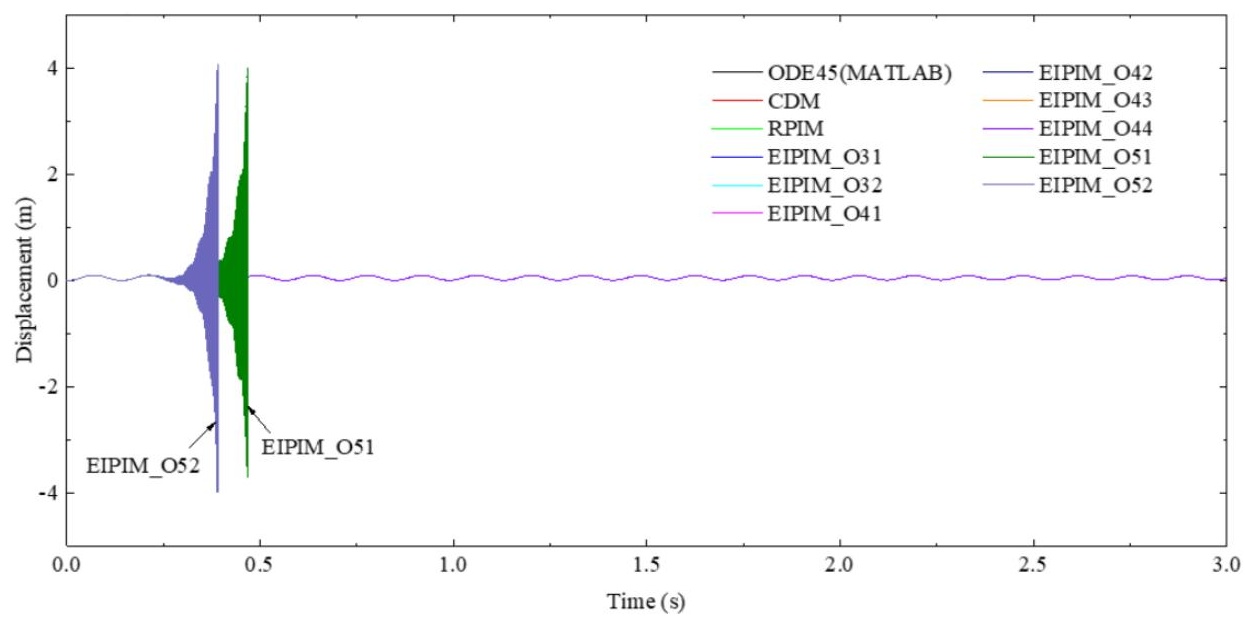

(a) Displacement curve with time increment $\Delta \mathrm{t}=0.00007 \mathrm{~s}$ 


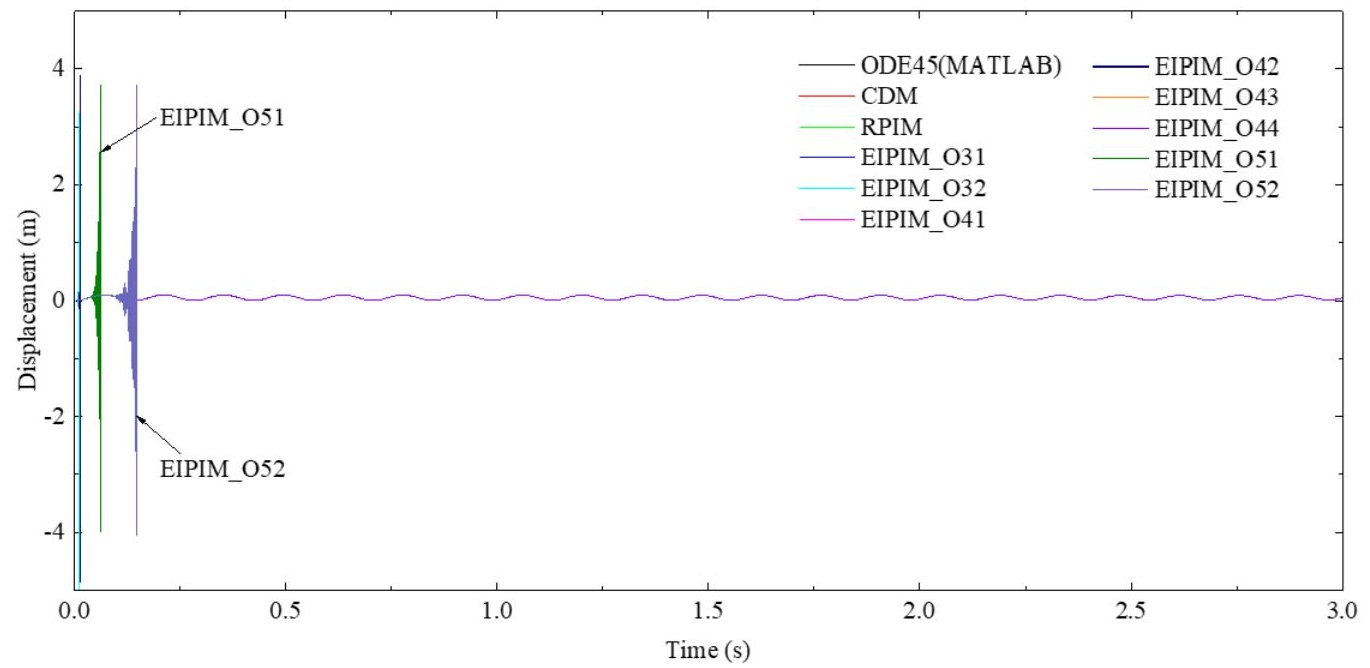

(b1) $0 \mathrm{~s} \sim 3.0 \mathrm{~s}$

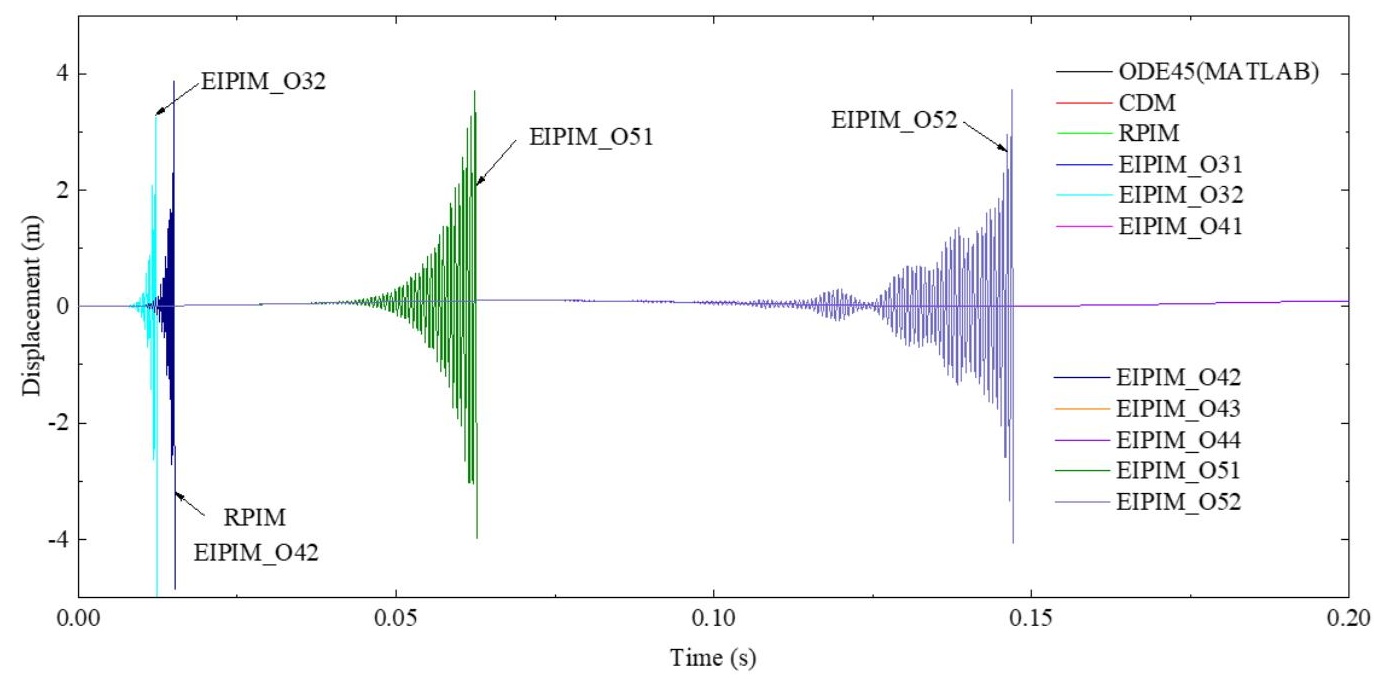

(b2) $0 \mathrm{~s} \sim 0.2 \mathrm{~s}$

(b) Displacement curve with time increment $\Delta \mathrm{t}=0.00011 \mathrm{~s}$

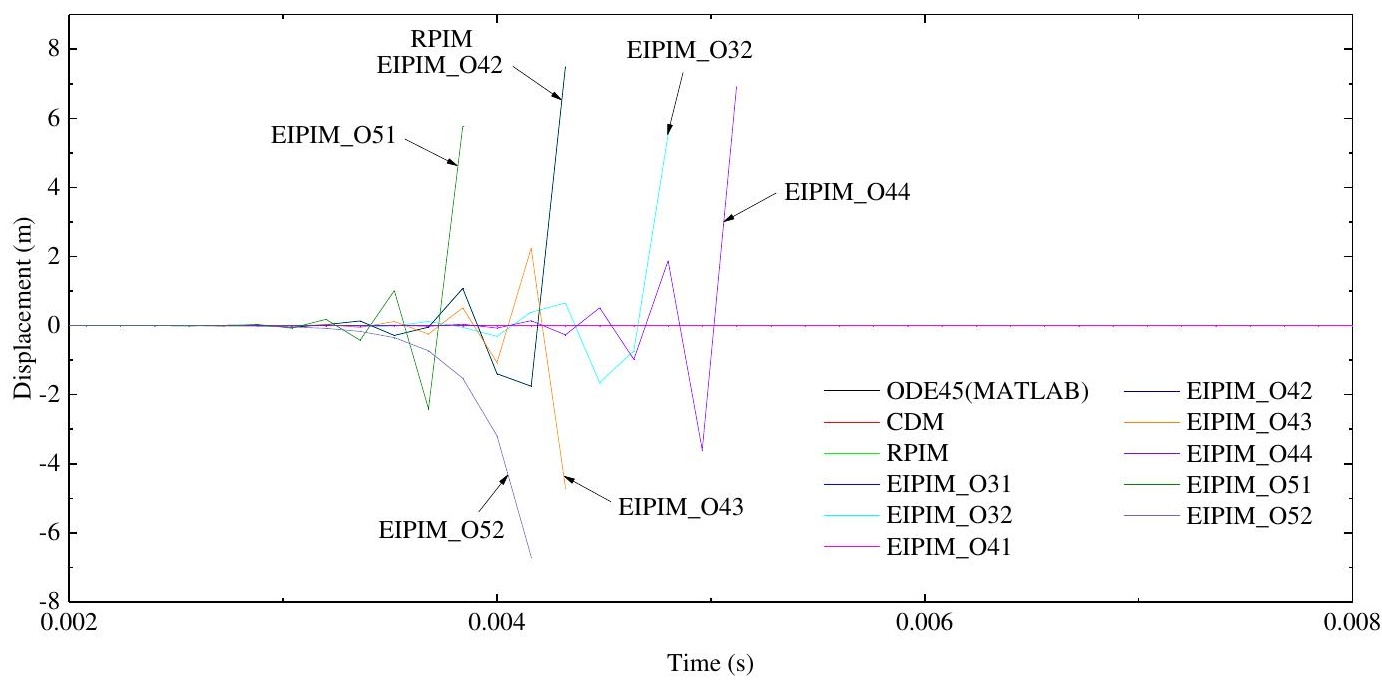

(c) Displacement curve with time increment $\Delta \mathrm{t}=0.00016 \mathrm{~s}$ 


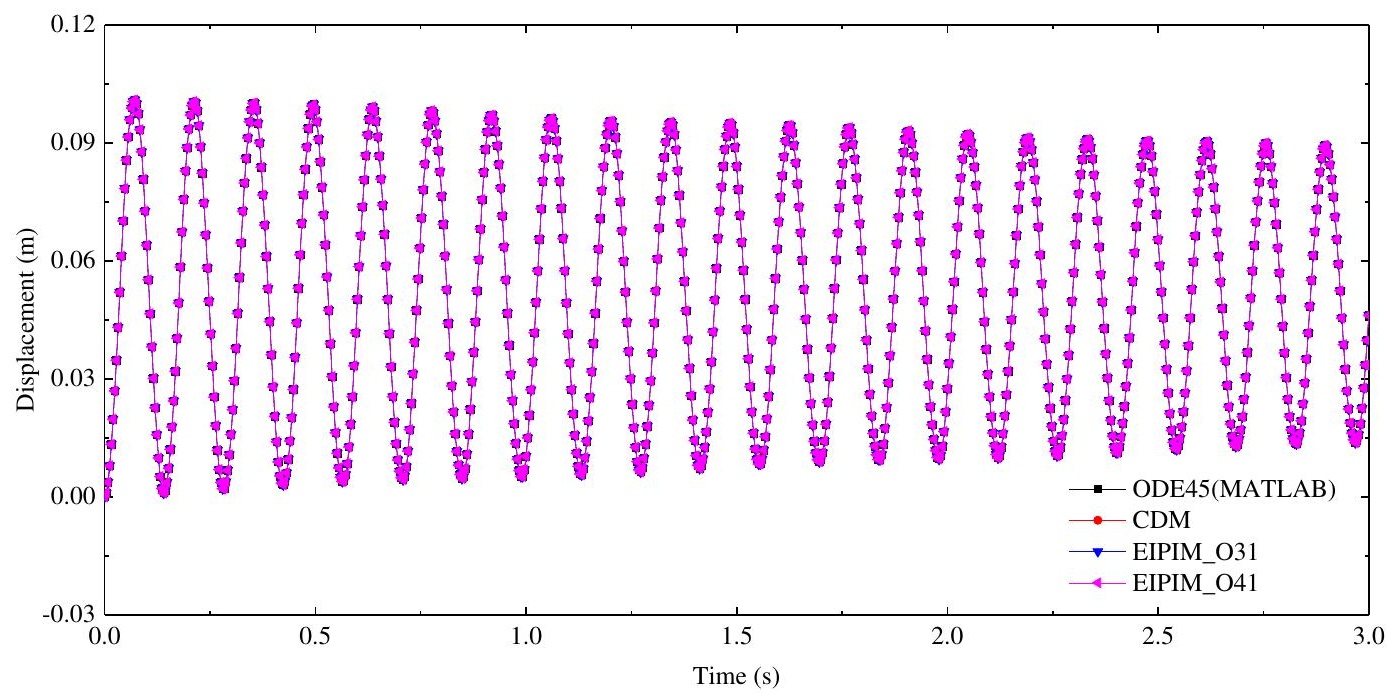

(d) Displacement curves of EIPIM_O31 and EIPIM_O41 with time increment $\Delta \mathrm{t}=0.00016 \mathrm{~s}$

Fig. 5 Time-displacement curves of the cantilever with constant force

A varied external force with the same amplitude of load in constant force case multiplying sine function $\sin (8 \pi \mathrm{t})$ is adopted in this part to test the stability and accuracy of EIPIMs in regard to a varied external force.

At time increment $\Delta \mathrm{t}=0.00005 \mathrm{~s}$, as shown in Fig. 6(a), the EIPIM_O51 and EIPIM_O52 provide a reasonable estimation of displacements at the beginning and start to lose stability at about $1.8 \mathrm{~s}$ with largely oscillated results from about $2.2 \mathrm{~s}$, demonstrating the ill-condition of higher order interpolation method again. The same as in free vibration and vibration with constant force, the RPIM starts to be ineffective from time increment $\Delta \mathrm{t}=0.00011 \mathrm{~s}$ and yields instability at the very beginning (Fig. 6(b)), while the EIPIM_O31 and EIPIM_O41 still provide accurate results when $\Delta \mathrm{t}=0.00016 \mathrm{~s}$ (Fig. 6(d)).

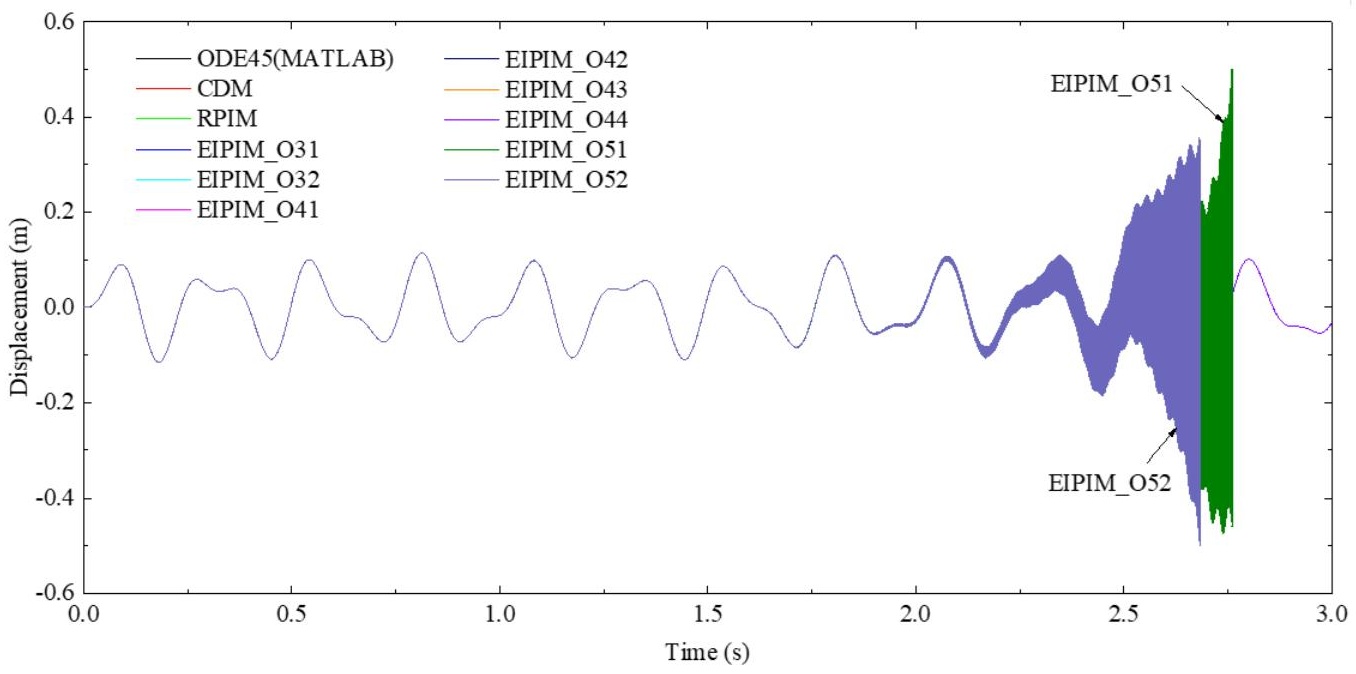

(a) Displacement curve with time increment $\Delta \mathrm{t}=0.00005 \mathrm{~s}$

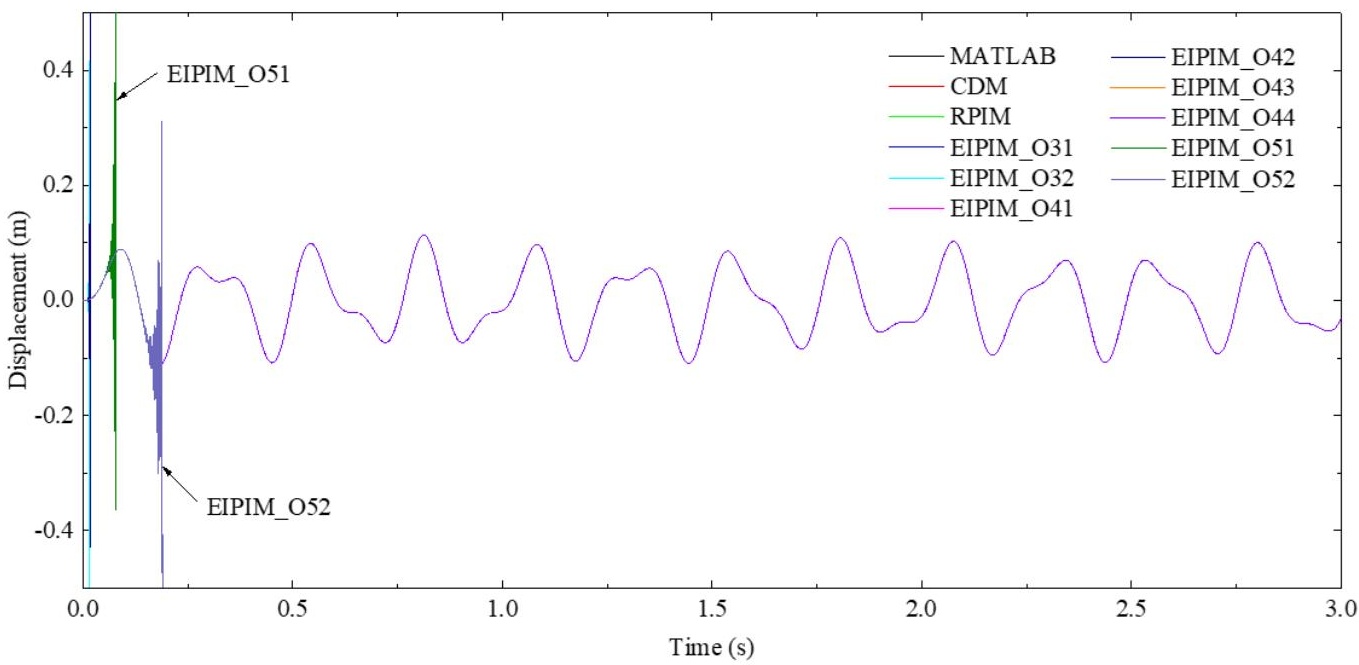

(b1) $0 \mathrm{~s} \sim 3.0 \mathrm{~s}$ 


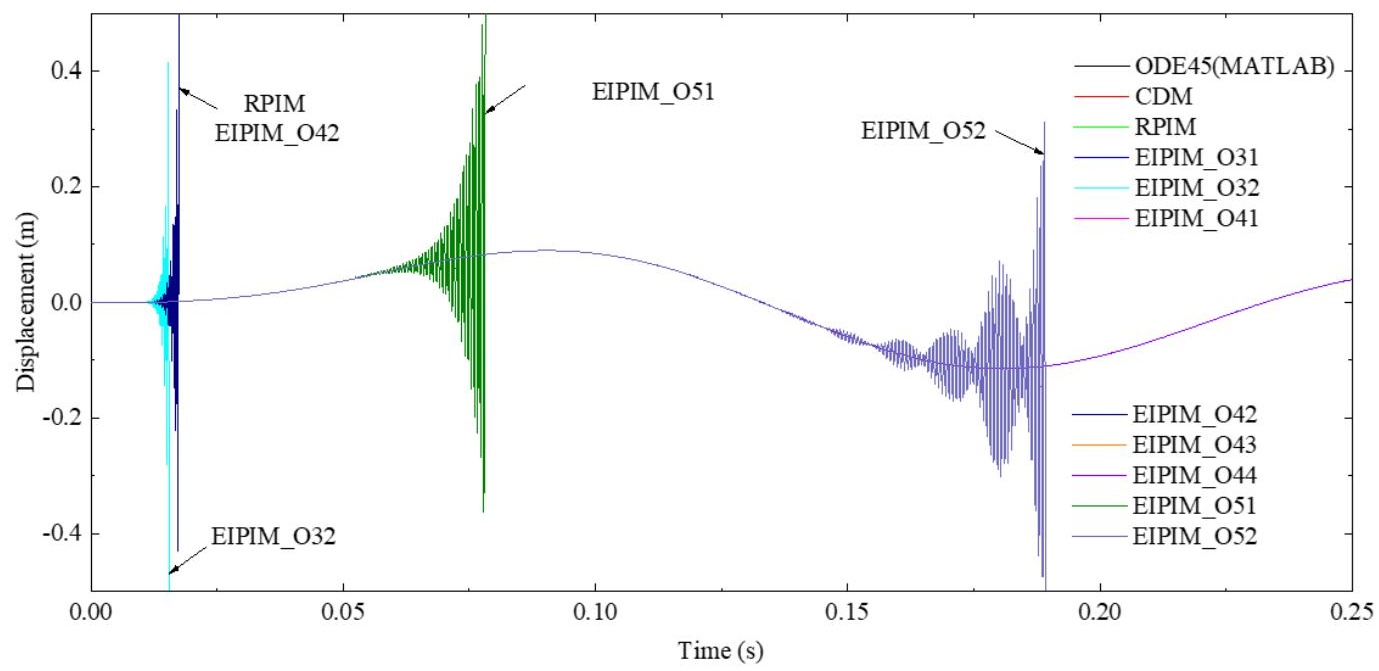

(b2) $0 \mathrm{~s} \sim 0.25 \mathrm{~s}$

(b) Displacement curve with time increment $\Delta \mathrm{t}=0.00011 \mathrm{~s}$

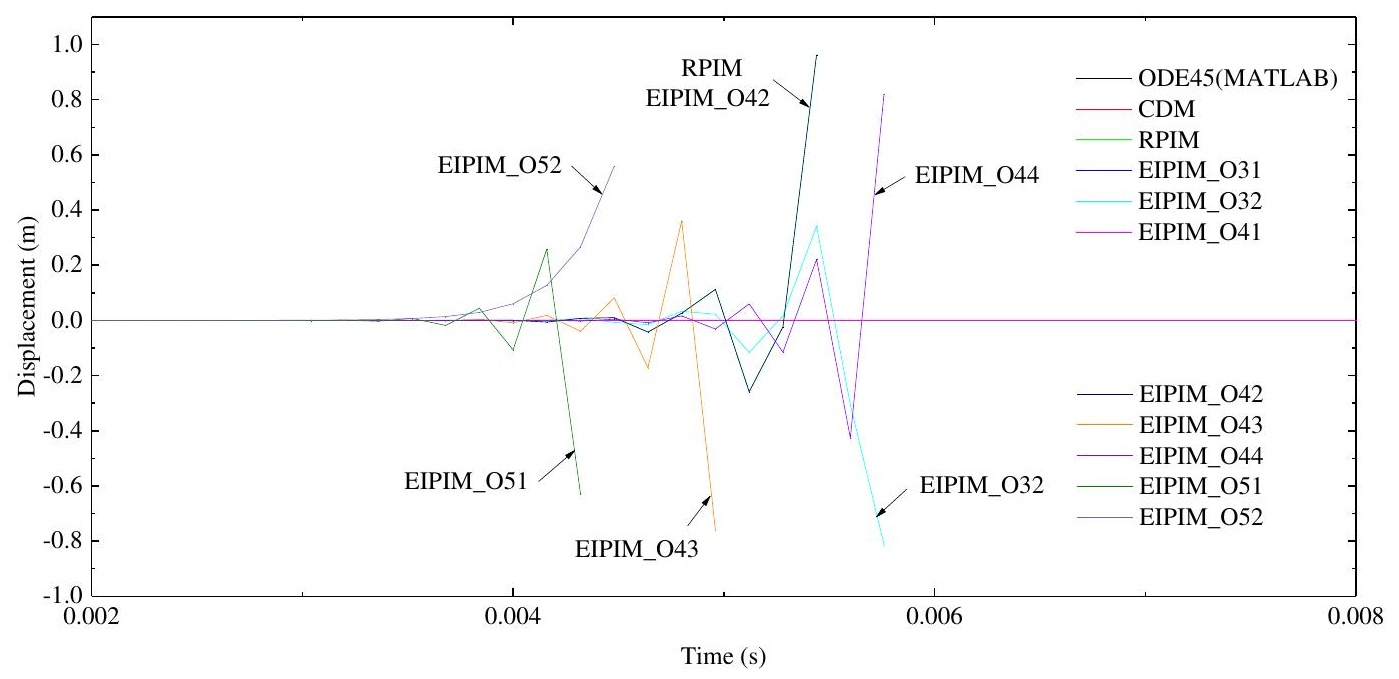

(c) Displacement curve with time increment $\Delta \mathrm{t}=0.00016 \mathrm{~s}$

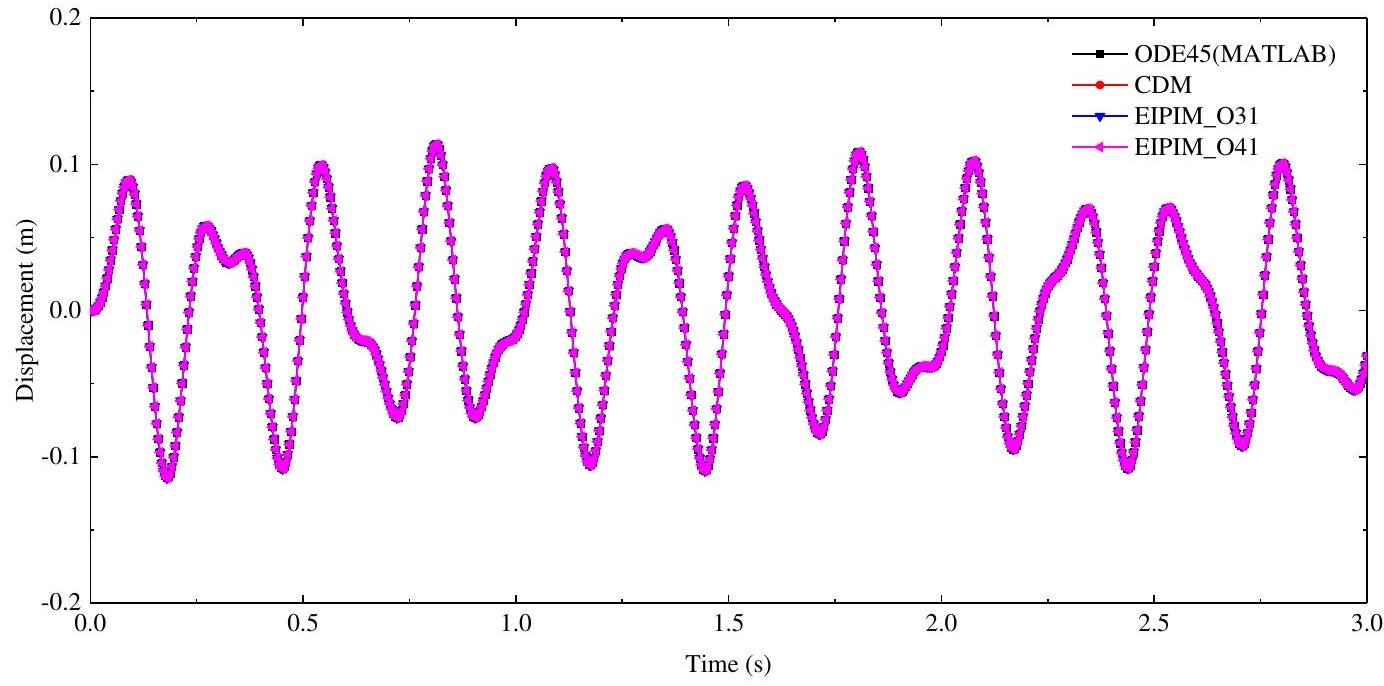

(d) Displacement curves of EIPIM_O31 and EIPIM_O41 with time increment $\Delta \mathrm{t}=0.00016 \mathrm{~s}$

Fig. 6 Time-displacement curves of the cantilever with sine force

The stable time limits of different methods in free vibration forced vibration with constant force, and varied force are illustrated in Fig. 7; 'EIPIM_' is omitted for EIPIMs to show the tendency of stability clearly. The same stable time limits are observed for each method, except little differences in EIPIM_O44, EIPIM_O51, and EIPIM_O52, demonstrating the reliability of
EIPIMs in dealing with different forms of external loads and initial displacements of the same structure. Among all the EIPIMs, methods with fifthorder accuracy (EIPIM_O51 and EIPIM_O52) exhibit lower stability than the original RPIM, and EIPIM_O32 has a little lower stability than RPIM. EIPIM_O31 and EIPIM_O41 have the highest stability, 60\% percent higher 
than the RPIM, while the EIPIM_O44 and EIPIM_O43 have relative higher stability. Conclusions could be drawn that estimation function with fifth order accuracy and Lagrange interpolation method (EIPIM_O32 and EIPIM_O42) are not suitable to be incorporated in EIPIM. Higher order interpolation method exhibits certain ill-condition as discussed earlier, and the Lagrange interpolation method only employs the displacements, which lacks the evaluation with velocity and acceleration. In summary, EIPIM_O31 and EIPIM_O41 are recommended for linear dynamic analysis due to their excellent stability.

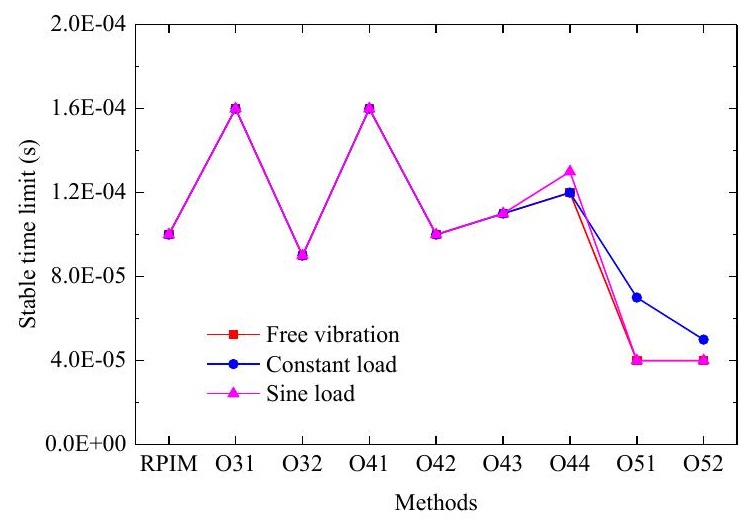

Fig. 7 Stable time limits of different methods for cantilever

The normalized CPU time of the RPIM, EIPIM_O31, and EIPIM_O41 at corresponding stable time limits for free vibration, forced vibration with constant force, and sine force are listed in Table 1.

Table 1

Comparison of CPU time of RPIM, EIPIM_O31, and EIPIM_O32

\begin{tabular}{ccccc}
\hline Methods & $\begin{array}{c}\text { Stable time limit } \\
(\mathrm{s})\end{array}$ & $\begin{array}{c}\text { Free } \\
\text { vibration }\end{array}$ & $\begin{array}{c}\text { Constant } \\
\text { force }\end{array}$ & $\begin{array}{c}\text { Sine } \\
\text { force }\end{array}$ \\
\hline RPIM & 0.0001 & 1.00 & 1.00 & 1.00 \\
EIPIM_O31 & 0.00016 & 0.50 & 0.67 & 0.68 \\
EIPIM_O41 & 0.00016 & 0.65 & 0.75 & 0.70 \\
\hline
\end{tabular}

As seen in Table 1, about 60\% CPU time is consumed by the EIPIM_O31 and EIPIM_O41 compared with the RPIM for free vibration and about $70 \%$ for forced vibration with constant force and sine force. The efficiency of EIPIM_O31 and EIPIM_O41 is superior to the RPIM benefiting from the increased stable time limits. It should also be noted that CPU time of EIPIM_O41 for each case is a little longer than that of EIPIM_O31, and this is because accelerations are required to be calculated in each step while there is no necessity for EIPIM_O31.

\subsection{Nonlinear dynamic analysis of truss}

In this example, a two-dimensional truss with two DOFs (i.e., horizontal and vertical displacements) for each node [43-44] is shown in Fig. 8. It is modeled by the truss element with geometric nonlinearity considered. The truss is fixed at two ends and external load $\mathrm{P}=2000 \mathrm{kN}$ is applied at nodes $1 \sim 7$ simultaneously, remaining constant during the analysis. The section and material information are listed as, section area $\mathrm{A}=0.01 \mathrm{~m} 2$, elastic modulus $E=2.06 \times 1011 \mathrm{~N} / \mathrm{m} 2$, density $\rho=7870 \mathrm{~kg} / \mathrm{m} 3$. Its layout and load pattern are illustrated in Fig. 8.

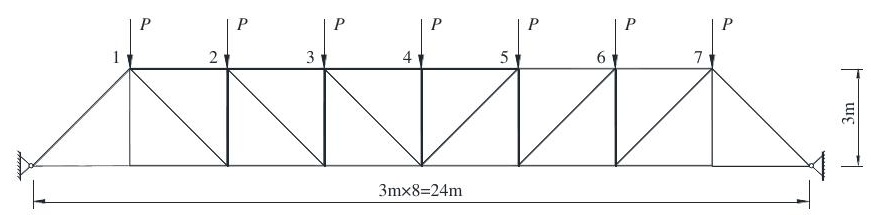

Fig. 8 Loading and boundary conditions of truss

The displacement at node 4 is monitored and the curves at different time increments are shown in Fig. 9. At time increment $\Delta t=0.00025$ s (Fig. 9(a)), the EIPIM_O51 and EIPIM_O52 cannot remain stable, while all other methods provide satisfying results. When time increment increases to $0.00045 \mathrm{~s}$ (Fig. 9(b)), the RPIM can no longer remain stable and yields oscillation from about 0.06s. At this time increment, EIPIM_O32 and EIPIM_O42, the two methods with Lagrange interpolation, are unstable as well. It is noteworthy that results provided by the RPIM and EIPIM_O42 vary, differing from the same results by RPIM and EIPIM_O42 in cases with linear elastic problems (section 6.1 and 6.2), resulting from the changing stiffness matrix during the nonlinear dynamic analysis. Nevertheless, not many differences are observed with regard to the starting time of instability or the tendency of discrepancy. The EIPIM_O31 and EIPIM_O41 are able to give reasonable results with satisfying accuracy at time increment $\Delta \mathrm{t}=0.00065 \mathrm{~s}$ (Fig. $9(\mathrm{~d})$ ), $62.5 \%$ higher than the stable time $\Delta \mathrm{t}=$ 0.0012 s of RPIM., when other methods all lose effectiveness (Fig. 9(c)).

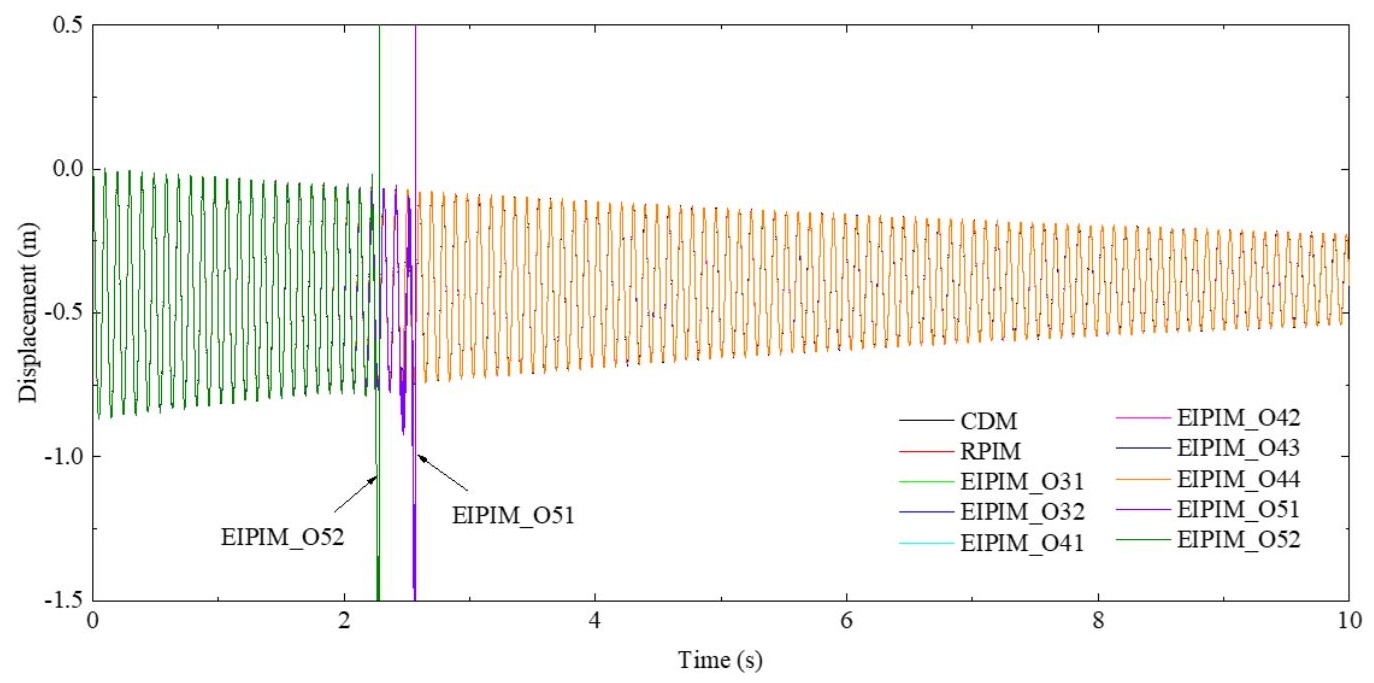

(a) Displacement curve with time increment $\Delta \mathrm{t}=0.00025 \mathrm{~s}$ 


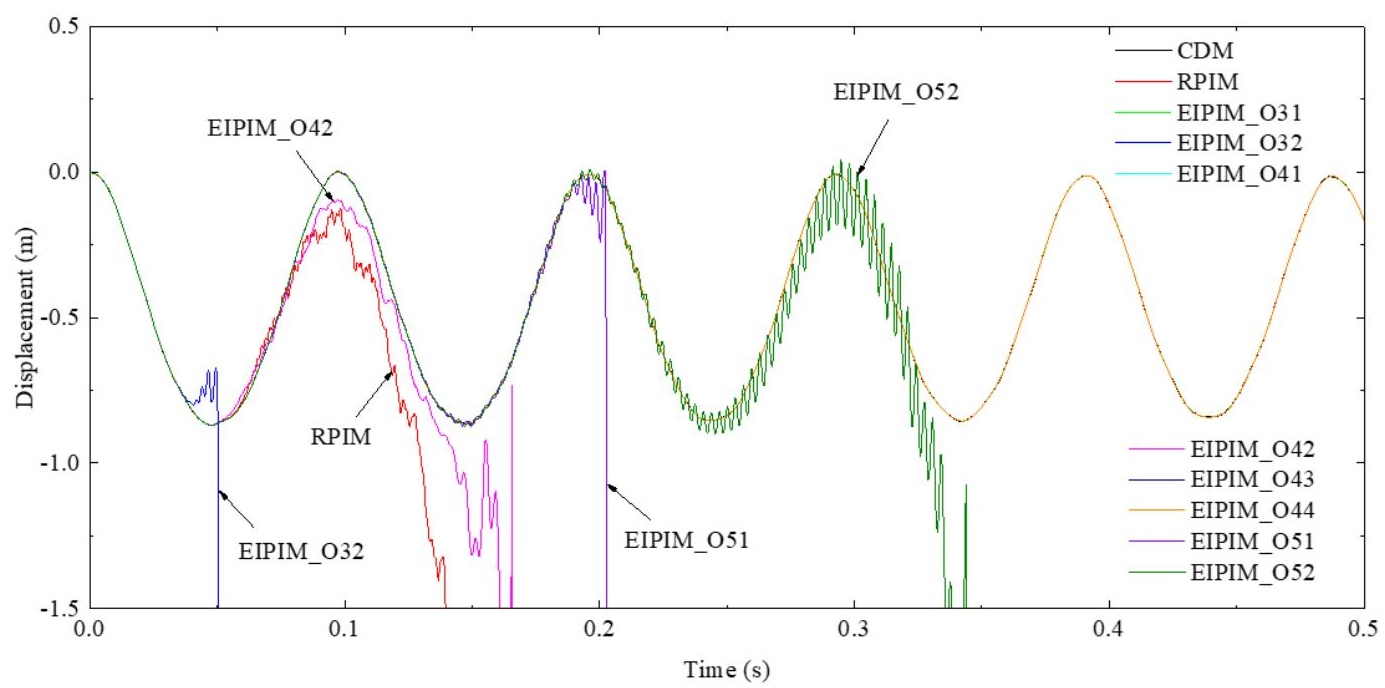

(b) Displacement curve with time increment $\Delta \mathrm{t}=0.00045 \mathrm{~s}$

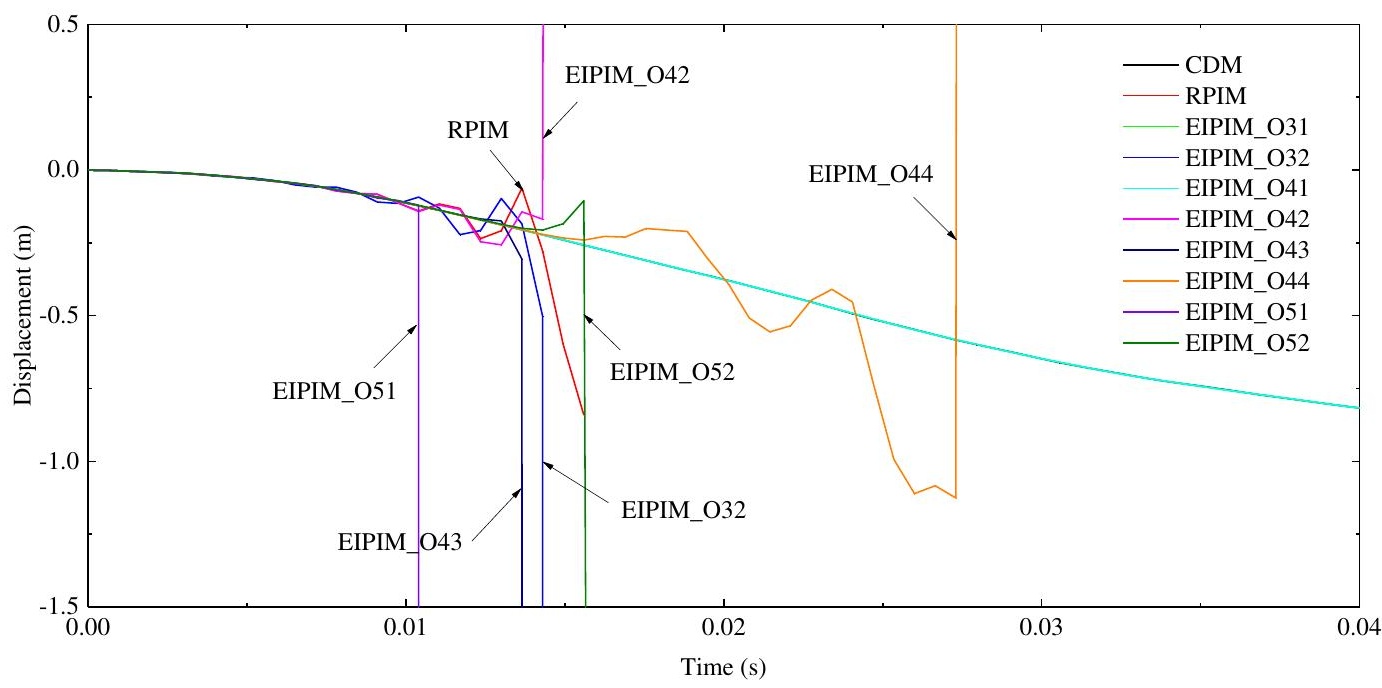

(c) Displacement curve with time increment $\Delta \mathrm{t}=0.00065 \mathrm{~s}$

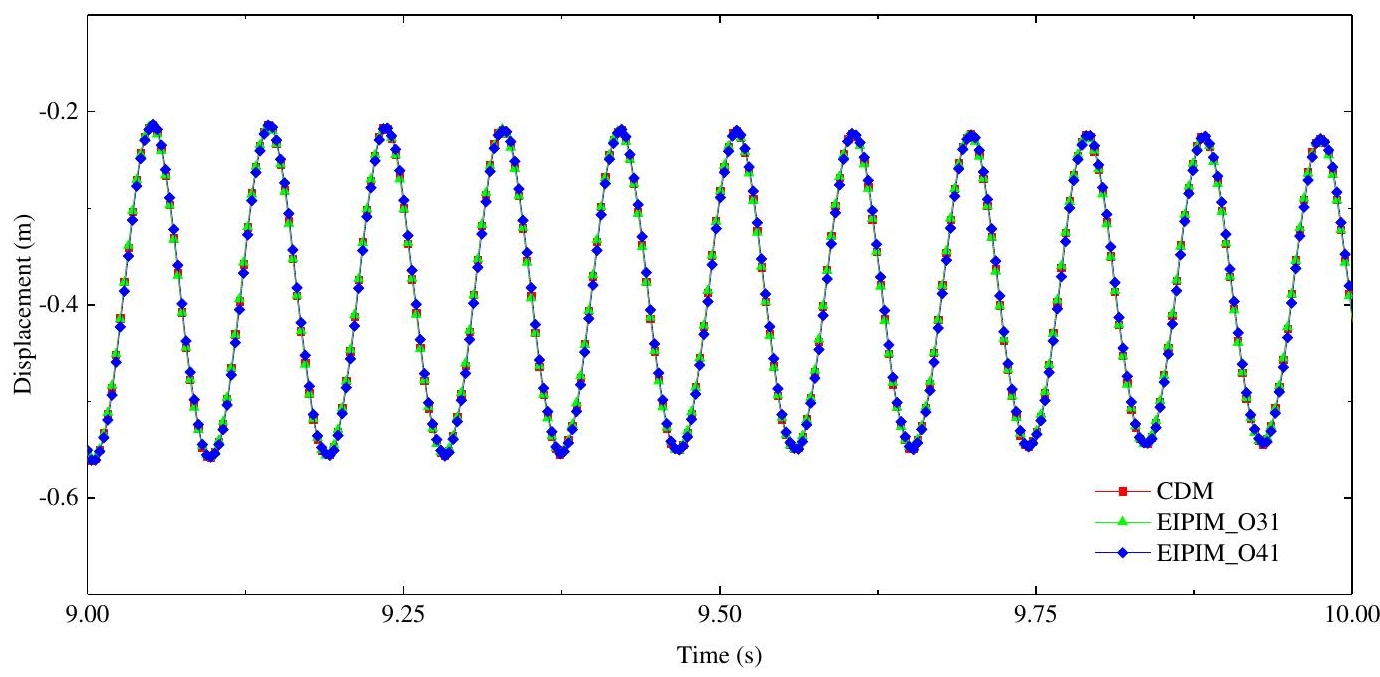

(d) Displacement curves of EIPIM_O31 and EIPIM_O41 with time increment $\Delta \mathrm{t}=0.00065 \mathrm{~s}$

Fig. 9 Time-displacement curves of truss with different methods

Stable time limits of each method for the nonlinear truss are illustrated in Fig. 10, and the 'EIPIM ' is also omitted as in Fig. 7 for simplicity. As can be seen, methods with fifth order accuracy exhibit lower stable time limits than the RPIM, while the stability of EIPIM_O32 and EIPIM_O42 with Lagrange interpolation method is close to that of RPIM. Similar to the linear elastic problem, EIPIM_O31 and EIPIM_O41 exhibit the most excellent stability with stable time limit 0.00065 s. EIPIM_O43 and EIPIM_O44 possess the same stable time limit $0.00045 \mathrm{~s}$, a little higher than that of RPIM. In this case, EIPIM O31 and EIPIM_O41 are the most suitable methods for nonlinear dynamic analysis of trusses. 


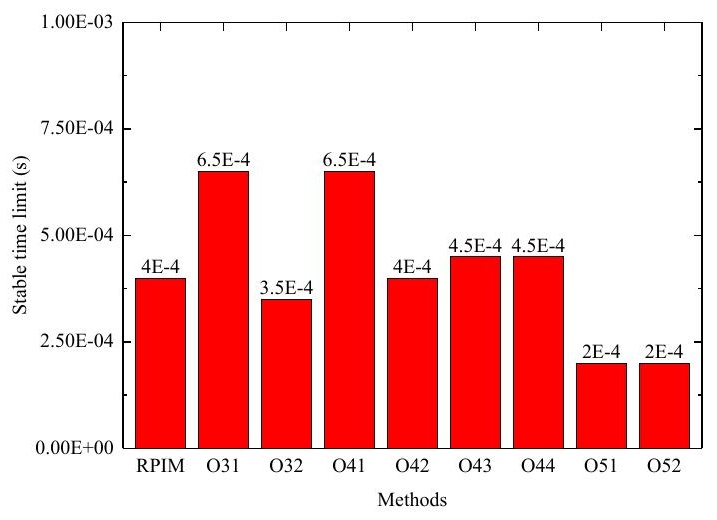

Fig. 10 Stable time limits of different methods for nonlinear truss

Differing from linear cases, CPU time of EIPIM_O31 and EIPIM_O41 at stable time limit $0.0065 \mathrm{~s}$ is about $85 \%$ and $90 \%$ respectively of that consumed by the RPIM at stable time limit $0.0004 \mathrm{~s}$. Additional estimation of the stiffness matrix is involved in nonlinear cases compared with linear cases, which is timeconsuming. Nevertheless, the increased computational effort is compensated by a larger stable time limit, demonstrating the efficiency of EIPIM_O31 and EIPIM_O41 for nonlinear analysis.

\subsection{Nonlinear analysis of Mindlin plate}

In this verification example, the shell element based on Mindlin plate theory [43] with geometric nonlinearity is employed to model a plate as shown in Fig. 11. The four-node shell element with two-point Gauss integration for bending and one-point Gauss integration for shear is adopted with the shear correction factor 5/6. For each node, there are five DOFs, two in-plane translation, one out-plane translation and two rotations [44]. In Fig. 11, a $2 \mathrm{~m} \times 2 \mathrm{~m}$ square plate with ten by ten mesh configuration is employed, with consistent thickness $\mathrm{t}=0.1 \mathrm{~m}$. It is subjected to uniform pressure $\mathrm{P}=100 \mathrm{kN} / \mathrm{m} 2$, remaining constant during the nonlinear dynamic analysis. Simple boundary condition with constrained translations and free rotations is employed at nodes along the four external sides. Elastic material with $E=2.06 \times 108 \mathrm{~N} / \mathrm{m} 2$, Poisson's ratio $\nu=0.3$, and density $\rho=7870 \mathrm{~kg} / \mathrm{m} 3$ is assumed.

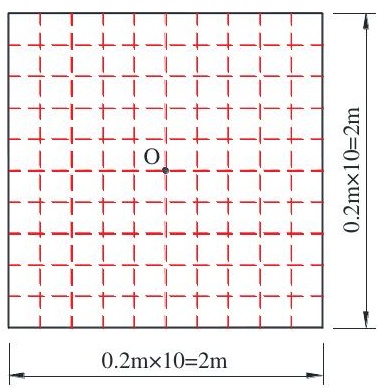

Fig. 11 Configuration of plate

The displacements of the center point $\mathrm{O}$ is monitored at different time increments to obtain the stable step limit of each method. Time-displacement curves at selected time increments are illustrated in Fig. 12. From this figure, EIPIM_O51 and EIPIM_O52 suffer instability at a small time increment $\Delta \mathrm{t}=$ $0.0003 \mathrm{~s}$, whereas other methods including RPIM are effective with enough accuracy (Fig. 12(a)). Nevertheless, RPIM is not able to provide stable results when time increment is raised to $0.00055 \mathrm{~s}$, as well as EIPIM_O32 and EIPIM_O42, the two methods with Lagrange interpolation estimation (Fig. 12(b)). When time increment augments $0.0008 \mathrm{~s}$, reasonable results are still available from EIPIM_O31 and EIPIM_O41, as shown in Fig. 12(d), while all the other methods yield to instability, as shown in Fig. 12(c). Conclusions can be drawn that EIPIM_O31 and EIPIM_O41 are the two methods with better stability and accuracy, while EIPIMs with the fifth-order accuracy estimation (EIPIM_O51 and EIPIM_O52) are unsuitable for nonlinear dynamic problems of plates.

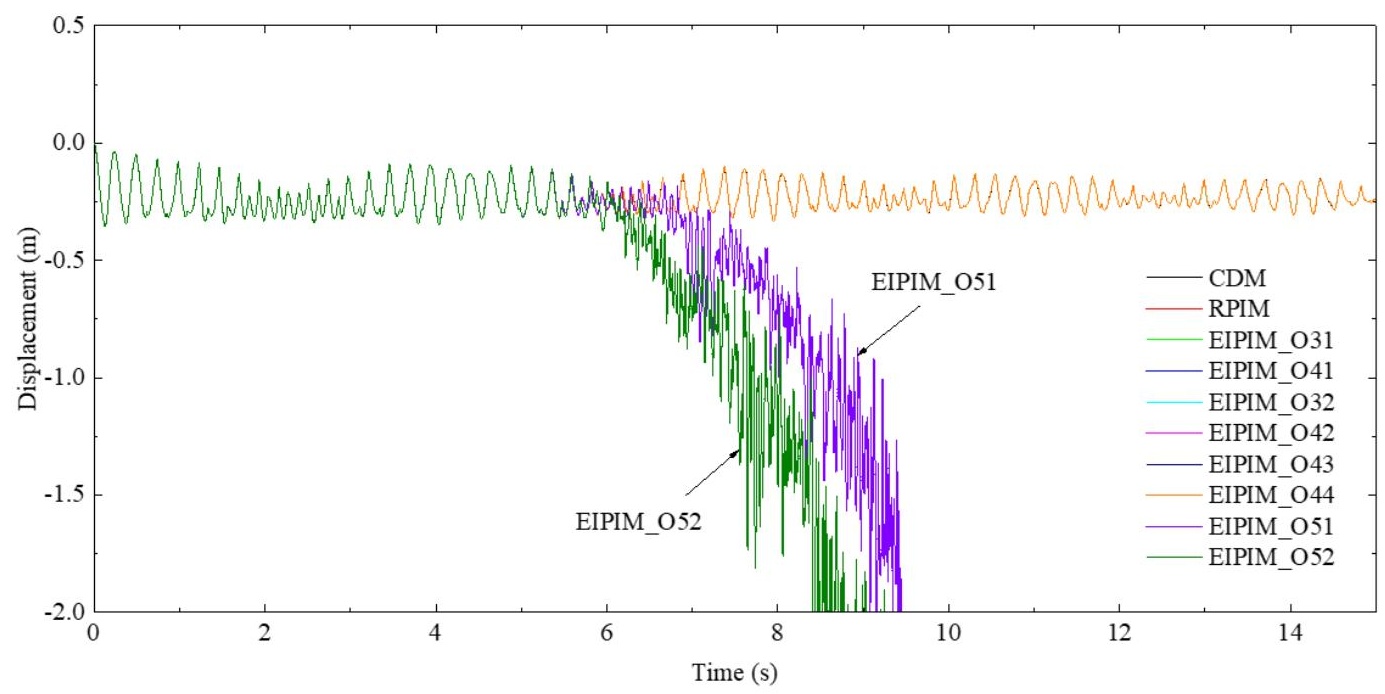

(a) Displacement curve with time increment $\Delta \mathrm{t}=0.0003 \mathrm{~s}$ 


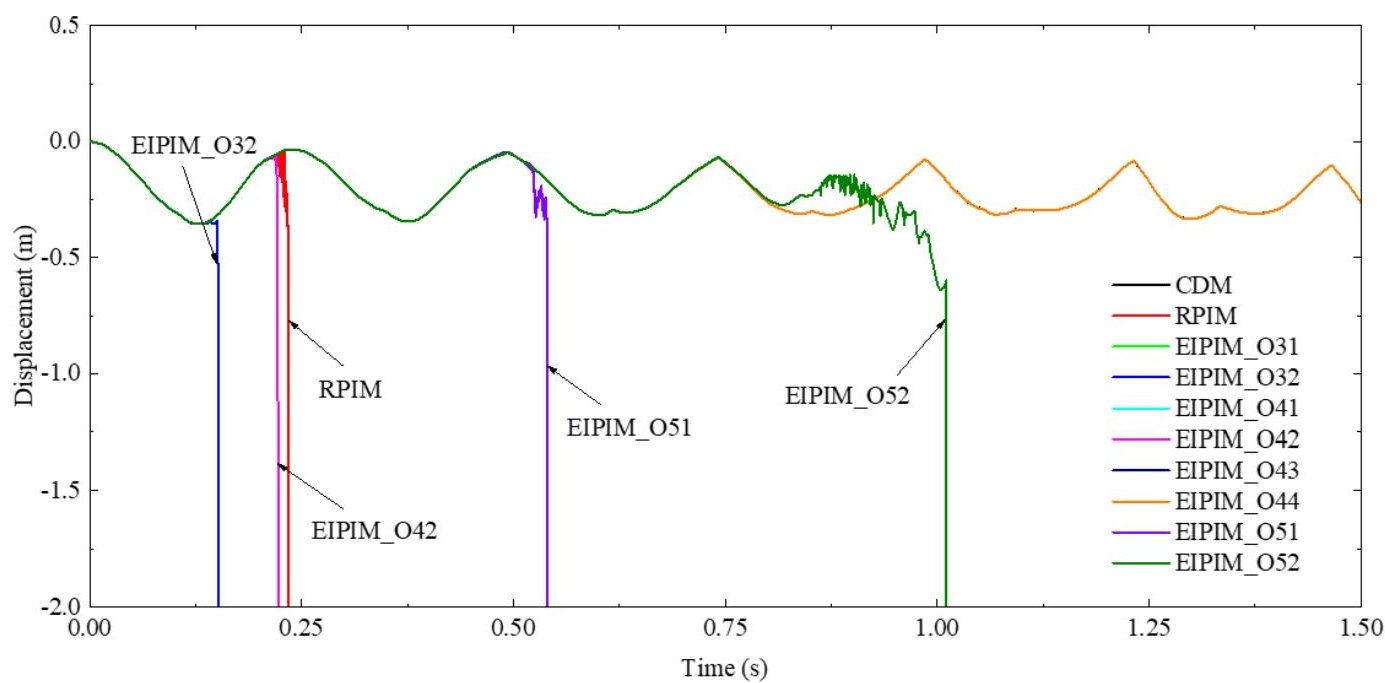

(b) Displacement curve with time increment $\Delta \mathrm{t}=0.00055 \mathrm{~s}$

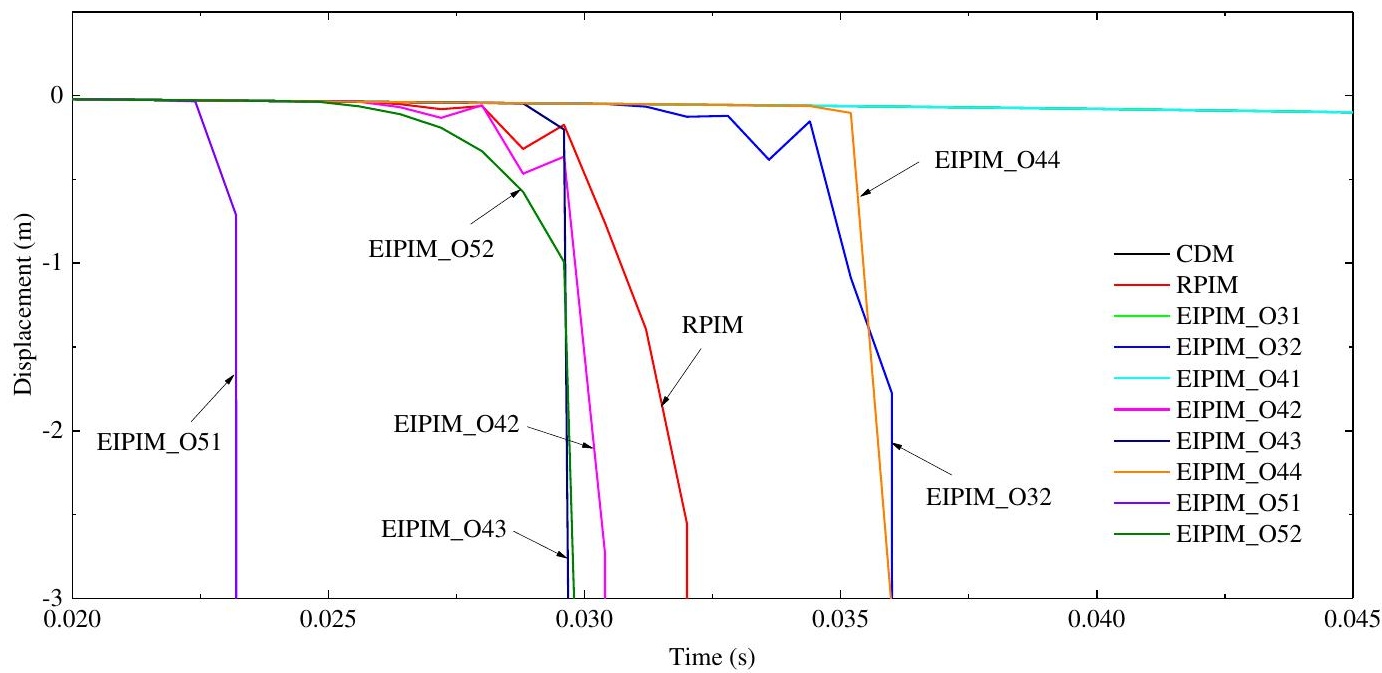

(c) Displacement curve with time increment $\Delta \mathrm{t}=0.0008 \mathrm{~s}$

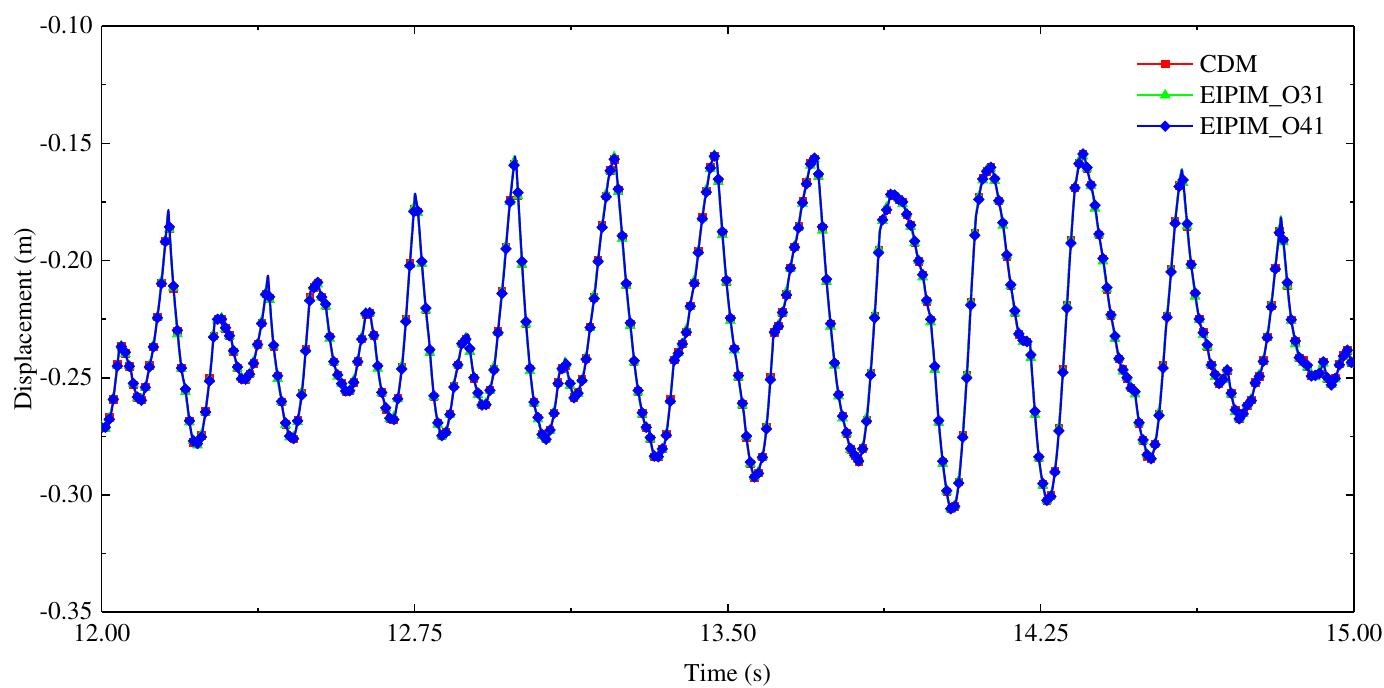

(d) Displacement curves of EIPIM_O31 and EIPIM_O41 with time increment $\Delta \mathrm{t}=0.0008 \mathrm{~s}$

Fig. 12 Time-displacement curves of the plate with different methods

To obtain an accurate stable time limit for each method, the time increments are enlarged gradually for all methods with the sub-increment $0.00005 \mathrm{~s}$. The stable time limits for each method are figured with 'EIPIM' omitted as in earlier cases, as shown in Fig. 13. Similar to linear cantilever and nonlinear truss, EIPIM_O51 and EIPIM_O52 have the smallest stable time limit, followed by RPIM, EIPIM_O32, and EIPIM_O42. The EIPIM_O43 and EIPIM_O44 have about $20 \%$ higher stable time increment than the RPIM. Meanwhile, EIPIM_O31 and EIPIM_O41 possess the highest stable time limit, 60\% higher than that of RPIM, indicating their applicability in nonlinear dynamic analysis of structures. 


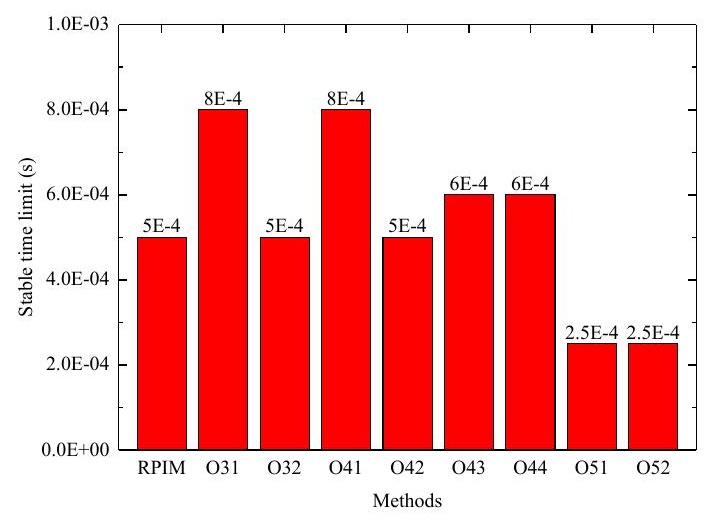

Fig. 13 Stable time limits of different methods for nonlinear plate

It is noteworthy that CPU time of EIPIM_O31 and EIPIM_O41 at the stable time limit is about $80 \%$ of that consumed by RPIM at stable time limit $0.0004 \mathrm{~s}$, lower than that in the case with nonlinear truss. More computational time could be saved with special procedures to calculate the stiffness matrix since the estimation of the stiffness matrix is the additional part in EIPIMs compared with RPIM. High efficiency of EIPIM_O31 and EIPIM_O41 is achieved with a larger stable time limit for nonlinear analysis, in despite of more computationally demanding in each step.

\section{Conclusions}

The refined precise integration method (RPIM) has been used for nonlinear dynamic analysis, but it has potential risk due to the numerical instability problem when using large time steps. In this paper, an improved method named the explicit-implicit precise integration method (EIPIM) is proposed based on the conventional RPIM. Eight explicit formulas including second-order, thirdorder and fifth-order functions have been studied in the framework of the new method. In general, EIPIM has the advantage of better numerical stability and higher precision than the traditional RPIM, and therefore, it has wider applications than RPIM in practical use. Several typical examples demonstrate

\section{References}

[1] He, Y.J., Zhou, X.H. and Wang, H.S., "Wind-induced response analysis of the cylindrical reticulated mega-structures", Advanced Steel Construction, 12, 66-82, 2016.

[2] Li Z, Ren H, Tong X, and Li H. , "A Precise Computation Method of Transient Free Surface Green Function", Ocean Engineering, 105, 318-326,2015.

[3] Zhong W.X., "On precise time integration method for structural dynamics", Journal of Dalian University of Technology, 34, 131-136, 1994.

[4] Zhong W.X. and Williams F.W., "A precise time step integration method", Proceedings of the Institution of Mechanical Engineers, Part C: Journal of Mechanical Engineering Science 208, 427-430, 1994

[5] Gao Q., Tan S.J. and Zhong W.X., "A survey of the precise integration method", Scientia Sinica Technologica, 46, 1207-1218, 2016

[6] Wu F., Gao Q. and Zhong W.X., "Subdomain Precise Integration Method for Periodic Structures", Shock and Vibration, 2014.

[7] Shen W.P., Lin J.H. and Williams F.W., "Parallel computing for the high precision direct integration method", Computer Methods in Applied Mechanics and Engineering, 126, 315$331,1995$.

[8] Gao Q., Wu F., Zhang H.W., Zhong W.X., Howson W.P. and Williams F.W., "A fast precise integration method for structural dynamics problems", Structural Engineering and Mechanics, 43, 1-13, 2012.

[9] Lin J.H., Shen W.P. and Williams F.W., "A high precision direct integration scheme for sturctures subjected to transient dynamic loading", Computers and Structures, 56, 113-120, 1995.

[10] Wang M.F. and Au F.T.K., "Assessment and improvement of precise time step integration method", Computers and Structures, 84, 779-786, 2006

[11] Wang M.F. and Au F.T.K., "Precise integration methods based on Lagrange piecewise interpolation polynomials", International Journal for Numerical Methods in Engineering, 77, 998-1014, 2009.

[12] Zhang,H.W., Zhang X.W. and Chen J.S., "A new algorithm for numerical solution of dynamic elastic-plastic hardening and softening problems" Computers and Structures, 81, $1739-1749,2003$

[13] Ding Z.X., Du Z.L., Liu Y.P. and Chan S.L., "A refined precise integration method for nonlinear dynamic analysis of structures", Advanced Steel Construction, 16, 124-136, 2020.

[14] Zhong W.X., "On precise integration method", Journal of Computational and Applied Mathematics, 163, 59-78, 2004.

[15] Gao Q., Wu F., Zhang H.W., Lin J.H. and Zhong W.X., "A fast precise integration method for large-scale dynamic structures", Chinese Journal of Computational Mechanics, 28, 492498, 2011.

[16] Bathe K.J. and Baig M.M.I., "On a composite implicit time integration procedure for nonlinear dynamics", Computers and Structures, 83, 2513-2524, 2005.

[17] Abaqus 6.17 Documentation. DS SIMULIA Corp, 2017.

[18] The Open System for Earthquake Engineering Simulation. Univeristy of California, Berkeley, 2000 the numerical stability and computational efficiency of the proposed EIPIM in both linear and nonlinear dynamic analysis.

Several conclusions can be drawn from this study as follows.

(1) EIPIM with higher order interpolation functions does not always perform as well as the lower order ones. For example, the fifth-order EIPIM_O51 and EPIM_O52 exhibit worse stability than RPIM due to the ill-condition of high order interpolation functions.

(2) EIPIMs with Lagrange interpolation functions such as EIPIM_O32 and EIPIM_O42 are not better than RPIM in terms of numerical stability and result precision because only displacements are adopted for the estimation in the next step. EIPIM_O42 provides the same results as RPIM for linear dynamic problems because they are essentially the same, while the different results are obtained from nonlinear dynamic cases due to the variable stiffness matrix.

(3) When fourth-order functions are adopted in the EIPIM, such as EIPIM_O43 and EIPIM_O44, EIPIMs show better performance than RPIM because of the improved accuracy of displacements adopted for the next step.

(4) EIPIM_O31 and EIPIM_O41 exhibit excellent numerical stability in solving both linear and nonlinear dynamic problems; with the time step about $60 \%$ larger than RPIM. Thus, the two methods allowing for large time steps are recommended for dynamic analysis of structures.

(5) Generally, EIPIM with Hermit interpolation functions (i.e. EIPIM_O31, EIPIM_O41, EIPIM_O43 and EIPIM_O44) is better than RPIM because the velocities and accelerations (if any) also follow the interpolation procedure used in displacements at each time step, except EIPIM with fifthorder functions due to the ill-condition of higher order interpolation method. The following is an example of two consecutive single-line equations written according to the specifications:

\section{Acknowledgments}

The authors are grateful for financial support from "Science and Technology Planning Project of Guangdong Province (2016A050502022) " and the Research Grant Council of the Hong Kong SAR Government on the project "Joint-based second order direct analysis for domed structures allowing for finite joint stiffness (PolyU 152039/18E)".

[19] Newmark N. M., "A Method of Computation for Structural Dynamics,” ASCE, 127, 1406 $1432,1962$.

[20] Wilson E.L., Farhoomand I. and Bathe K.J., "Nonlinear dynamic analysis of complex structures", Earthquake Engineering Structural Dynamics, 3, 241-252, 1972.

[21] Wilson E.L., A Computer Program for the Dynamic Stress Analysis of Underground Structure, 1968

[22] Mohammad R.P. and Mahdi K.R., "A New Explicit Time Integration Scheme for Nonlinear Dynamic Analysis," International Journal of Structural Stability and Dynamics, 16, 1550054, 2015

[23] Du X.Q., Yang D.X., Zhou J.L, Yan X.L., Zhao Y.L. and Li S., "New Explicit Integration Algorithms with Controllable Numerical Dissipation for Structural Dynamics", International Journal of Structural Stability and Dynamics, 18, 1850044, 2018.

[24] Bathe K.J., "Conserving energy and momentum in nonlinear dynamics: A simple implicit time integration scheme", Computures and Structures, 85, 437-445, 2007.

[25] Bathe K.J. and Noh G., "Insight into an implicit time integration scheme for structural dynamics", Computures and Structures, 98, 1-6, 2012

[26] Noh G. and Bathe K.J., "Further insights into an implicit time integration scheme for structural dynamics", Computures and Structures, 202, 15-24, 2018

[27] Zhang J., Liu Y.H. and Liu D.H., "Accuracy of a composite implicit time integration scheme for structural dynamics", International Journal for Numerical Methods in Engineering, 109, 368-406, 2017.

[28] Kim W. and Choi S.Y., "An improved implicit time integration algorithm: The generalized composite time integration algorithm", Computures and Structures, 196, 341-354, 2018.

[29] Zienkiewicz O.C. and Xie Y.M., "A simple error estimator and adaptive time stepping procedure for dynamic analysis", Earthquake Engineering and Structural Dynamics, 20, 871 $887,1991$.

[30] Ascher U.M., Ruuth S.J. and Spiteri R.J., "Implicit-explicit Runge-Kutta methods for timedependent partial differential equations", Applied Numerical Mathematics, 25, 151-167, 1997.

[31] Lai T, Yi T.H., Li H.N. and Fu X., “An Explicit Fourth-Order Runge-Kutta Method for Dynamic Force Identification", International Journal of Structural Stability and Dynamics, 17, 1750120, 2017.

[32] Hulbert G.M. and Jang I., "Automatic time step control algorithms for structural dynamics", Computer Methods in Applied Mechanics and Engineering, 50, 155-178, 1995.

[33] Deokar R., Maxam D. and Tamma K.K., "A novel and simple a posteriori error estimator for LMS methods under the umbrella of GSSSS framework: Adaptive time stepping in secondorder dynamical systems", Computer Methods in Applied Mechanics and Engineering, 334 414-439, 2018.

[34] Wang L. and Zhong H.Z, "A time finite element method for structural dynamics", Applied Mathematical Modelling, 41, 445-461, 2017.

[35] Mayr M., Wall W.A. and Gee M.W., "Adaptive time stepping for fluid-structure interaction solvers", Finite Elements in Analysis and Design, 141, 55-69, 2018

[36] Rossi D.F., Ferreira W.G., Mansur W.J. andCalenzani A.F.G., "A review of automatic timestepping strategies on numerical time integration for structural dynamics analysis", Engineering Structures, 80, 118-136, 2014

[37] Kuo S.R., Yau J.D. and Yang Y.B., "A robust time-integration algorithm for solving 
nonlinear dynamic problems with large rotations and displacements", International Journal of Structural Stability and Dynamics, 12, 1250051, 2012.

[38] Liu Y. and Shen W.P., "Adaptive algotithm of transition matrix in precise integration method", Journal of Vibration and Shock, 2, 82, 1995.

[39] Tan S.J., Gao Q. and Zhong W.X., "Applications of Duhamel term's precise integration method in solving nonlinear differential equations", Chinese Journal of Computational Mechanics, 25, 752-758, 2010

[40] Alipour A. and Zareian F., "Study Rayleigh Damping in Structures; Unceratinties and Treatments", The 14th World Conference on Earthquake Engineering, Beijing, China, 2008. [41] Bathe K.J. and Wilson E.L., "Stability and accuracy analysis of direct integration methods", Earthquake Engineering and Structural Dynamics, 1, 3-6, 1983.

[42] MATLAB Documentation, MathWorks, 2000.

[43] Bathe K.J., Finite element procedures in engineering analysis, Prentice-Hall, Inc, New Jersey, 1982.

[44] Ferreira A.J.M., MATLAB codes for finite element analysis: solids and structures, Springer, 2008 


\title{
AN EXPERIMENTAL STUDY ON MECHANICAL PROPERTIES OF CONCRETE-FILLED STEEL TUBE (CFST) KEY-CONNECTED PREFABRICATED WALL AND COLUMN
}

\author{
Qian $\mathrm{Wu}^{1,2}$, Guo-Chang $\mathrm{Li}^{2,}{ }^{*}$, Ming $\mathrm{Li}^{2}$ and Yuan-Wei Luo ${ }^{1,2}$ \\ ${ }^{1}$ School of Civil Engineering, Dalian University of Technology, Dalian, Liaoning, 116024, china \\ ${ }^{2}$ School of Civil Engineering, Shenyang Jianzhu University, Shenyang, Liaoning, 110168, china \\ *(Corresponding author: E-mail:liguochang0604@sina.com)
}

\section{A B S T RA C T}

The prefabricated structures have the advantages of high construction speed, energy efficiency, environmental friendliness, and low labor demand. At present, the connection of fabricated concrete structural components mainly relies on connecting the stressed steel bars of the prefabricated components using a variety of methods, and then casting concrete or grout at the joints. This requires a large quantity of wet work, and most studies on this topic focused on frame structures and shear wall structures. The research on frame-shear wall structures is still rare. To fill this gap, a new type of connecting prefabricated shear wall and prefabricated frame column in the assembled frame-shear structure by using concrete-filled steel tubs (CFST) keys is proposed with experimental study. This paper analyzes the differences in the mechanical properties and the failure modes of three kinds of structures, i.e. the wall and column connected by CFST keys, the wall and column connected by the CFST keys with concrete block placed between them, and the wall and column connected by the cast-in-place method. This paper also investigates the mechanical properties and failure modes of the first kind of structure when the failure of shear wall is caused by the CFST keys. The test results show that the CFST keys with the reinforced concrete block placed between them are superior to the cast-in-place structures in terms of wall-column connection mode, and the bearing capacity and ductility of the structure, while sharing similar initial stiffness of the structure.The CFST keys connection mode shows engineering feasibility and good seismic performance. When designing this type of connection, the strength of the CFST keys should be moderately controlled. If the strength level is too high, the shear resistance of the overall structure will decrease.

\section{A R T I C L E H I S T O R Y}

$\begin{array}{ll}\text { Received: } & \text { 30 September } 2019 \\ \text { Revised: } & \text { 10 April 2020 } \\ \text { Accepted: } & \text { 18 April } 2020\end{array}$

\section{K E Y W O R D S}

Concrete-filled steel tube;

frame column;

shear wall;

Prefabricate

Frame-shear wall structure

\section{Introduction}

The prefabricated concrete structure has the advantages of high construction speed, good energy efficiency and environmental friendliness, and low labor demand. It has become a topic of common concern for civil engineers in various countries. Japan, the United States, France and other countries have given continuous attention to the prefabricated concrete structure. Among them, in Japan, 9 million prefabricated houses were built during the period 1963 to 2014 and about 150,000 houses were built each year in the last few years. Since the 1950s, the development of prefabricated buildings has started in the US, where a typical movable house can be completed by prefabrication (Yuan et al.[1], Si et al.[2]). In China, a large number of prefabricated buildings were made in the 1970s and 1980s. However, due to the limited level of science and technology at that time, there were engineering quality problems in these buildings, which were gradually replaced by cast-in-place structures. With the advancement of science and technology in the past ten years, rapid progress was witnessed in the development of prefabricated concrete structures in China, and a large number of such structures have been built throughout the country, achieving remarkable economic and social benefits. This fully demonstrates that the prefabricated buildings are one of the important development directions of the world's future buildings.

The development of prefabricated reinforced concrete structures is based on the cast-in-place ones, which has three important types: frame structure, shear wall structure, and frame-shear wall structure. The key technical issue of the fabricated concrete structures is to study how to realize the splitting and connection of the above-mentioned structures, and to ensure that the performance of the prefabricated structures is at least as good as that of the cast-in-place ones. In order to achieve this goal, scholars of various countries carried out a number of theoretical and experimental research. The research on the fabricated frame structure started earlier and has yielded numerous connection methods, of which the beam and column were connected initially by extending the steel bars from the end of the prefabricated members or by the bracket of the column (Restrepo et al. [3]). New development included the form of the main ribs of the beam and column connected by the sleeve grouting method ( $\mathrm{Li}$ et al. [4], $\mathrm{Li}$ et al.[5]) and the connection form using prestressed or high-strength steel bars (Alcocer et al.[6],Guan et al.[7], Zhao et al.[8]), both of which have achieved good results. The research on the fabricated shear wall structure started later (Lu et al.[9], Chen et al.[10]), but also produced a number of research findings. For example, different types of shear walls have been studied, including linear type, I-shaped, L-typed, and T-shaped. Several studies focused on the influence on the seismic behavior of these shear walls exerted by the following factors: the length of the splicing zone, the length of the ribs, the material of the splicing zone, and the form of the splicing zone(Sun et al.[11], Song et al.[12], LU et al.[13], JGJ101-96[14], Smith et al.[15], Pekau et al.[16], Soudki et al.[17], LI et al.[18], Soudki et al.[19]). Nevertheless, there are few studies on the prefabricated frame-shear structure. Compared with the frame and shear wall structure, the assembled frame-shear structure requires more research on the wall-to-column connection techniques and wall-to-beam connection techniques.

To this end, our research team proposed straight rib joint, straight rib U-shaped lap joint, U-shaped rib lap joint, and chain buckle lap joint to be used for the wall-column or beam-wall connection. The direct shear test and the quasi-static test with two-layer plane structure were carried out. The results showed that the mechanical properties of the latter two are better (Yuan.[1], Si.[2], Wang.[20], Gao.[21], Liang.[22]). This method is suitable for a single-storey construction condition where the wall and column are large. When the wall and column are small in a single-storey building, the wall, column and beam can also be prefabricated into a unit, and then assembled directly in a similar way to the frame and shear wall structure. Research has shown that this method can also be achieved satisfactory effects (Mo, et al.[23], Ma, et al.[24]).

As mentioned above, while various connection schemes have been proposed in the research and development of the assembly technology of the prefabricated concrete components, these schemes mainly use different methods to connect the stressed steel bars of the prefabricated components and then cast concrete or grout at the joints to realize the prefabrication. The connection between the components requires relatively heavy wet work at the joints and the construction quality is difficult to control (Wu et al.[25] and [26], Bin et al.[27], Ming et al.[28]). Therefore, some researchers have proposed the use of steel or steel tube concrete to join prefabricated concrete components, in order to reduce the amount of wet work and further increase the degree of industrialization. They also predicted that the form of the combined structure would likely become an ultimately accepted form in the development of prefabricated buildings. Based on this line of research, our research team proposed a new scheme of connecting prefabricated shear wall and prefabricated frame column in the assembled frame-shear structure with CFST keys, and analyzed its feasibility by finite element simulation method (Gao.[21], Liang.[22]). We continue to conduct experimental research in this paper. These findings will drive the application of composite structural 
components in fabricated concrete structures.

\section{Structure of wall and column connected with CFST keys}

The components and assembly structure of the CFST key-connected wall and column are shown in Fig.1. Fig.1 (a) is the embedded part, which is composed of anchor steel plate, bottom steel plate and shear steel plate. Fig.1 (b) is CFST key, and Fig.1(c) is precast reinforced concrete block (the internal stirrup is 3A8@100, the internal longitudinal reinforcement is $4 C 18$. andindicate the yield strength of the bars is $300 \mathrm{Mpa}$ and $400 \mathrm{Mpa}$, respectively (GB50017-2017[27]). Fig.1(d) is a split diagram of the embedded part. Fig.1(e) is a schematicdiagramof the structureof the CFST key-connected wall and column.

The construction sequence is as follows: Firstly, the prefabricated wall and the prefabricated column are placed in predeterminateposition. After the CFST keys are placed between the prefabricated column and the prefabricated wall, the two ends are welded on the bottom steel plate of the embedded part in the prefabricated wall and the prefabricated column. Lastly, the reinforced concrete block is placed between the CFST keys. The gapsamong the CFST keys, the reinforced concrete block, the prefabricated wall, and the prefabricated column are filled with grout. In this process, to meet the installation requirements, the height of the CFST keys should be slightly smaller than the spacing of the shear-resistant steel plate of the embedded part; and the length of the CFST keys should be slightly smaller than the distance between the fabricated column and the fabricated wall. The construction error of the part can be filled by the weld; The size of the reinforced concrete block should be slightly smaller than the hole between the CFST keys and the prefabricated column and the prefabricated wall, and the gap can be filled with the grout.

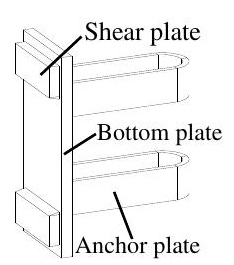

(a)Embeddedpart

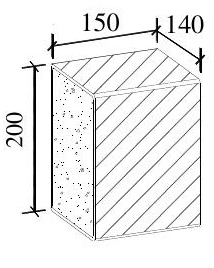

(b) CFST key

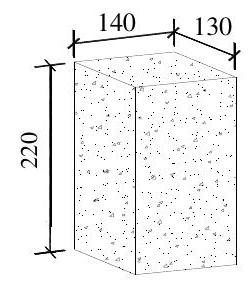

(c) Reinforced concrete block

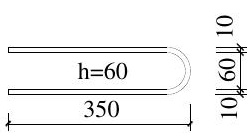

Anchor plate

Shear plateBottom plate

(d) Embedded parts split diagram

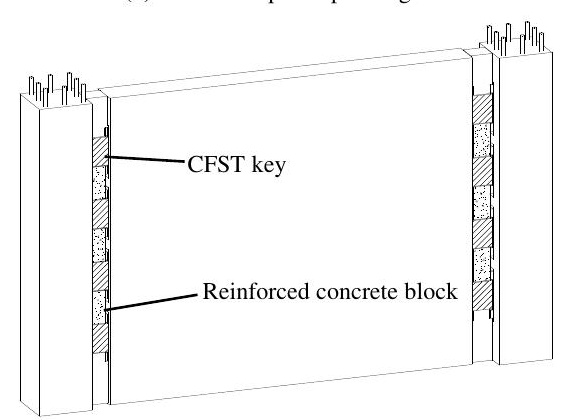

(e) Three-dimensional diagram of the structure with CFST key-connected wall and column

Fig. 1 Three-dimensional diagram of components and structure of the CFST key-connected wall and column

\section{Experimental program}

\subsection{Design and fabrication of specimen}

In the event of horizontal earthquake action, the prefabricated frame column and the prefabricated shear wall will move against each other. When this happens, the CFST keys will play a role of deformation coordination, bearing the shear force resulted from the movements between them. To study the effect of transmitted shear force, we conducted a two-stage experimental study. In the first stage, the failure of the CFST keys was controlled. The analysis was focused on the differences in the mechanical properties and the failure modes among three kinds of structures, i.e., the wall and column are connected by the CFST keys, the wall and column are connected by the CFST keys with concrete block placed between them, and the wall and column are connected by the cast-in-place method. We attempted to identify a wall-column connection scheme that meets the requirements. In the second stage, a condition was controlled to make sure that no or only minor failure of the CFST keys occur, while the connected wall failed.The focus was on the mechanical properties and failure modes of the shear wall caused by the CFST keys.

To complete the analysis of the first stage, we designed specimens S1, S2, and S3. In order to ensure the failure of the CFST keys, frame column components were designed at the two ends of the CFST keys of the specimens.The geometry and reinforcement of each specimen are shown in Fig.2. Specimen S1 was not equipped with reinforced concrete blocks between the CFST keys; Specimen S2 contained a reinforced concrete block between the CFST keys. Specimens S1 and S2 had the same reinforcement. Specimen S3 was a cast-in-place specimen with the connection area being a shear wall. The sizes of shear steel plates used in Specimens S1 and S2 were designed according to the shear force transmitted by the three-sided welded seam of the shear steel plate and the shear capacity of the CFST keys. The size of the CFST keys was determined based on the relevant literature(GAO.[21]). The wall thickness of the steel tube is $4 \mathrm{~mm}$. The dimensions of the embedded parts, CFST keys, and reinforced concrete blocks are shown in Fig.1.

To complete the analysis of the second stage, specimen S4 was designed. One end of the CFST keys of the specimen was connected to the frame column, and the other end was connected to the shear wall. In order to ensure that no damage or only minor damage to the CFST keys when the specimen wastested, the wall thickness of the steel tube was increased to $8 \mathrm{~mm}$. The geometrical dimensions and reinforcement of the specimen are shown in Fig.3. Specimen S4 shared some identical properties with specimens S1 to S3 in the dimensions of the CFST keys, the embedded part, the steel bars for the beam and column, the beam, and the column. The strength of concrete and the steel bar and the steel plate were also the same with those specimens. The steel strain gauge arrangement is shown in Fig.3(a), which uses $\operatorname{Si}(i=1,2,3, \ldots)$ to indicate the strain gauge number of the steel.

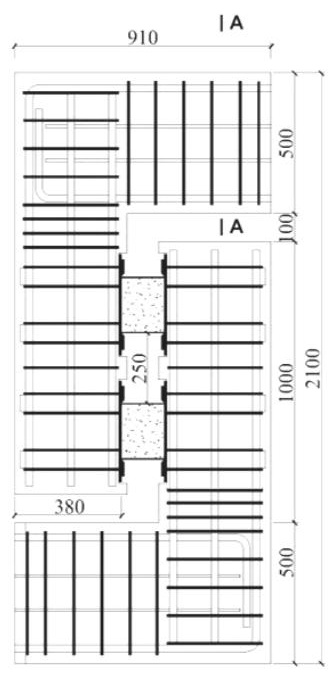

(a) Specimens S1 and S2

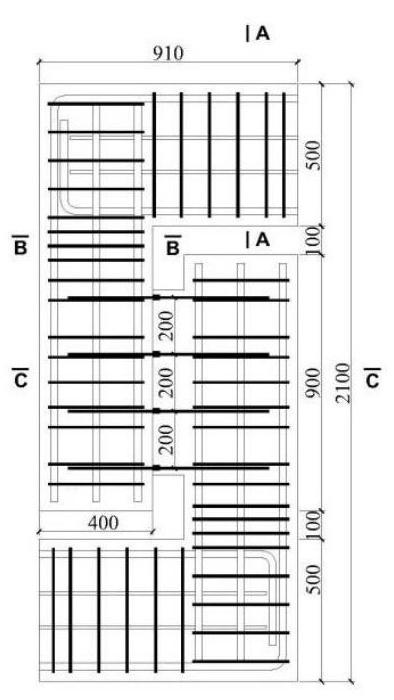

(b) Specimen S3

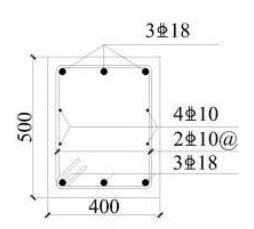

A-A

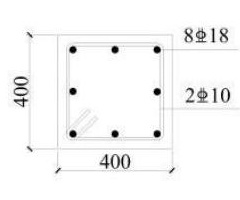

B-B

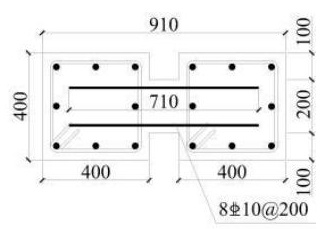

C-C
Fig. 2 Geometrical dimensions and reinforcement diagram of specimens S1, S2and S3 

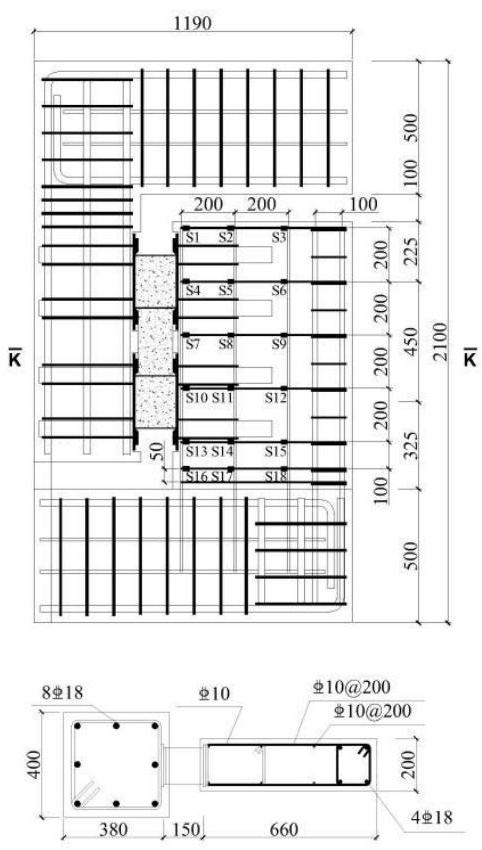

D-D

Fig. 3 Geometrical dimensions, reinforcement, and steel strain gauge arrangement of specimen $\mathrm{S} 4$

\subsection{Materials mechanical properties}

In the test, Chinese grade Q235B structural steel was used for all steel members (GB50017-2017 [29]), and Chinese grade C40structural concretewas used for all concrete members (GB50010-2010 [30]). Standard coupons were cut from each steel member and tested. The material properties of steel and concrete are listed in Table 1 , where $\varepsilon_{\mathrm{y}}, f_{\mathrm{y}}, f_{\mathrm{u}}, E_{\mathrm{s}}$ are steel yield strain, yield strength, tensile strength and elastic modulus respectively. Casting of $150 \times 150 \times 150 \mathrm{~mm}$ cubes for concrete strength $\left(f_{\text {ck }}\right)$ test and $150 \times 150 \times 300 \mathrm{~mm}$ cylinder for concrete Young's modulus $\left(E_{\mathrm{c}}\right)$ were carried out at the same time. They were cured in similar conditions as the specimen.

Table 1

Material mechanical properties of steel bar and concrete

\begin{tabular}{cccccc}
\hline Material & $\begin{array}{c}E_{\mathrm{s}} / E_{\mathrm{c}} \\
\left(\mathrm{N} / \mathrm{mm}^{2}\right)\end{array}$ & $\begin{array}{c}E_{\mathrm{y}} \\
(\mu \varepsilon)\end{array}$ & $\begin{array}{c}f_{\mathrm{y}} \\
(\mathrm{MPa})\end{array}$ & $\begin{array}{c}f_{\mathrm{u}} \\
(\mathrm{MPa})\end{array}$ & $\begin{array}{c}f_{\mathrm{ck}} \\
(\mathrm{MPa})\end{array}$ \\
\hline Steel bar (A8) & $1.4 \times 10^{5}$ & 3100 & 434 & 584 & - \\
Steel bar (C10) & $2.2 \times 10^{5}$ & 2400 & 528 & 604 & - \\
Steel bar (C14) & $1.7 \times 10^{5}$ & 2335 & 397 & 563 & - \\
Steel plate(t=4) & $2.1 \times 10^{5}$ & 1304 & 274 & 352 & - \\
Steel plate ( $\mathrm{t}=8)$ & $2.2 \times 10^{5}$ & 1686 & 371 & 501 & - \\
Steelplate $(\mathrm{t}=10)$ & $2.2 \times 10^{5}$ & 1695 & 362 & 489 & - \\
Concrete & $3.4 \times 10^{4}$ & - & - & - & 42 \\
\hline
\end{tabular}

\subsection{Experimental setup and loading procedure}

Fig. 4 shows the test loading device, the displacement gauge, and strain gauge layout for specimens S1 to S3. Fig.4(a) shows the $10000 \mathrm{kN}$ largescale mufti-functional structural testing machine system (WAW-J12000J); Fig.4(b) and Fig.4(c) show the displacement gauge and strain gauge layout of specimens S1, S2and S3, respectively. The top of the specimen was provided with a loading steel plate $(400 \mathrm{~mm} \times 300 \mathrm{~mm} \times 40 \mathrm{~mm})$. To prevent local pressure damage, a layer of fine sand was placed to the contact area between the loading steel plate and the specimen. The bottom of the specimen was placed directly on the test bench, and blocks were arranged on both sides. The two ends of the block were tightly attached to the steel beam anchored on the test bench to prevent the specimen from moving laterally. A jack was applied with a vertical load in contact with the loading steel plate, and the loading force center passes through the symmetry axis of the specimen. Strain gauges (Y1 to Y8) were arranged at the upper and lower surface ends of specimens S1, S2and the strain gauge rosettes ( $\mathrm{H} 1$ and $\mathrm{H} 2$ ) were arranged at the center of the side of the steel tube, as shown in Fig.4(b). Strain gauges (Y1 to Y8) were placed at the point where the column is connected with the horizontal steel bar on the wall for specimen S3, as shown in Fig.4(c). Horizontal displacement meters (W1 and W2) were placed on the side of specimens S1, S2and S3 to monitor whether the specimen moves horizontally. A vertical displacement meter (W3) was placed on the side of the specimen to monitor the vertical displacement of the specimen, as shown in Fig.4(b) and Fig.4(c). In addition, vertical displacement information was also collected by the test machine system.

Loading was performed by graded loading with each level set at $150 \mathrm{kN}$, each stage held for 1 to 2 minutes, and the loading speed controlled at 0.5 $\mathrm{mm} / \mathrm{min}(\mathrm{GB} / \mathrm{T}$ 50152-2012 [31]).

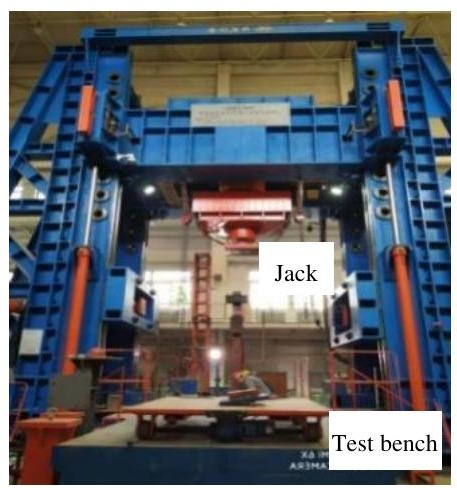

(a)Testing machine system

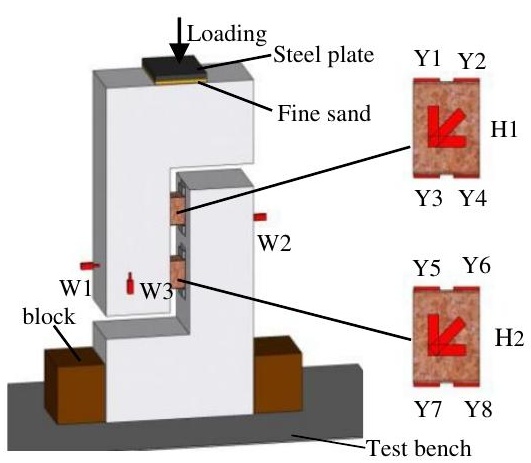

(b) Displacement gauge and strain gauge layout for specimens $\mathrm{S} 1$ and $\mathrm{S} 2$

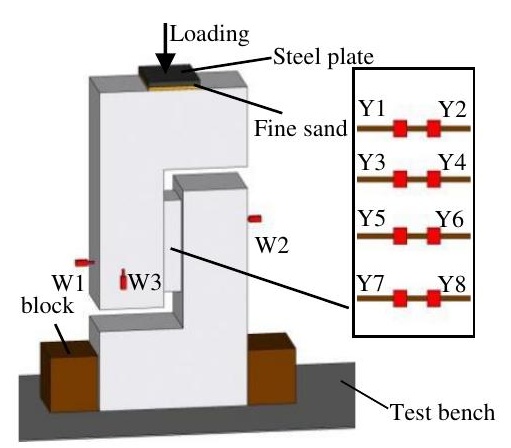

(c) Displacement gauge and strain gauge layout for specimen $\mathrm{S} 3$

Fig. 4 Test loading device, displacement gaugeandstrain gauge layout

When specimen S2 was tested by the above device, the junction area between the column end and the beam was broken because the reinforced concrete block was arranged between CFST keys of the specimen S2 and the shear bearing capacity was high. The first test failed, as shown in Fig.5(a). Therefore, the specimen was re-reinforced. The cracked concrete was removed and the grout was used for filling, as shown in Fig.5(b). To avoid the damage of this junction area, a reinforced test device was designed, as shown in Fig.6. That is, a steel beam was added on the side of the column; One end of the steel beam was fixed by the end steel plate and the tie rod, and the other end was fixed by the L-shaped fastener. One end of the fastener was connected to one side of the column by a thick steel plate, and the other end was connected to the end beam by a tie rod and a nut. With this reinforcing device, the test of the specimen S2 was successfully completed. 


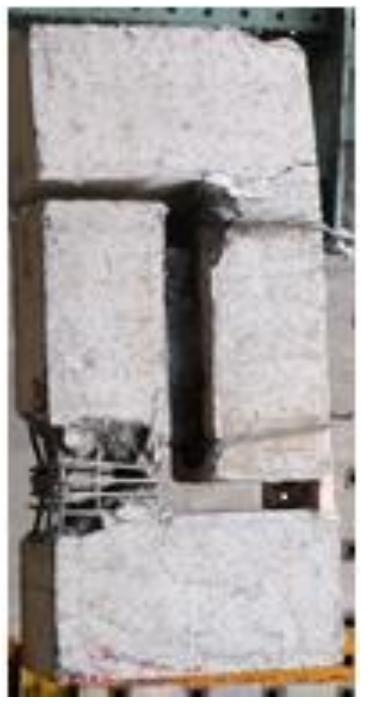

(a)Failed specimen

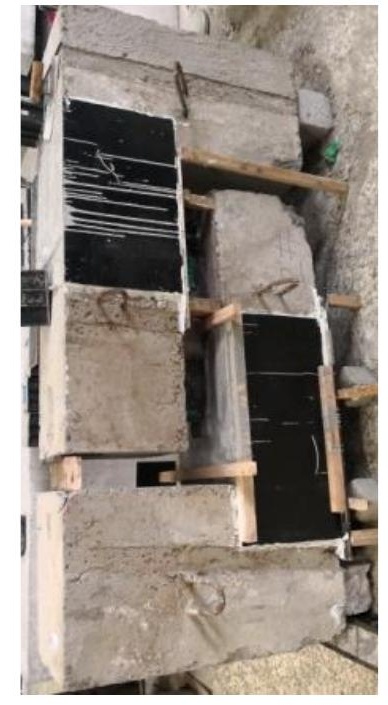

(b)Reinforcedspecimen
Fig. 5 Failed specimen and reinforced specimen

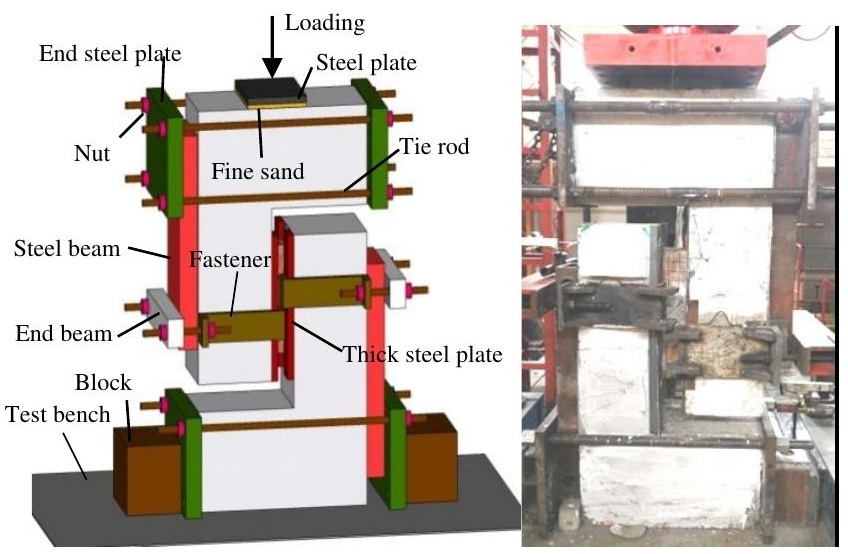

Fig. 6 Reinforced specimen and test device

In order to ensure that the beam-column junction area does not break which leads to the failure of a test, a column reinforcement method was applied directly to the loading device for specimen S4, as shown in Fig. 6. The positions of the displacement gauges (W1, W2, W3, and W4), the strain gauges (G1 to G8), and the strain gauge rosettes (GH1 and GH2) of the CFST keys are also indicated in Fig.7. Specimen S4 was loaded with the center of the force through the center of the CFST keys.

\section{Experimental results and discussions}

\subsection{Comparison of load-displacement curves}

Fig.8 is a load-vertical displacement curve of each specimen. For the convenience of analysis, the following variables were calculated: The initial stiffness $E_{0}$ of each specimen (ratio of vertical load to the according displacement in the elastic phase), yield load $P_{\mathrm{y}}$ (calculated with the "universal yield bending moment method"), maximum load $P_{\max }$, ultimate load $P_{\mathrm{u}}$ (85\% of maximum load), and the corresponding displacement ductility coefficient $\mu$ (the ratio of the ultimate load to the corresponding displacement of the yield load) (Bin etal.[27], Ming etal.[28], GB50017-2017 [29], Li et al .[32]). The results are as shown in Table 2 .

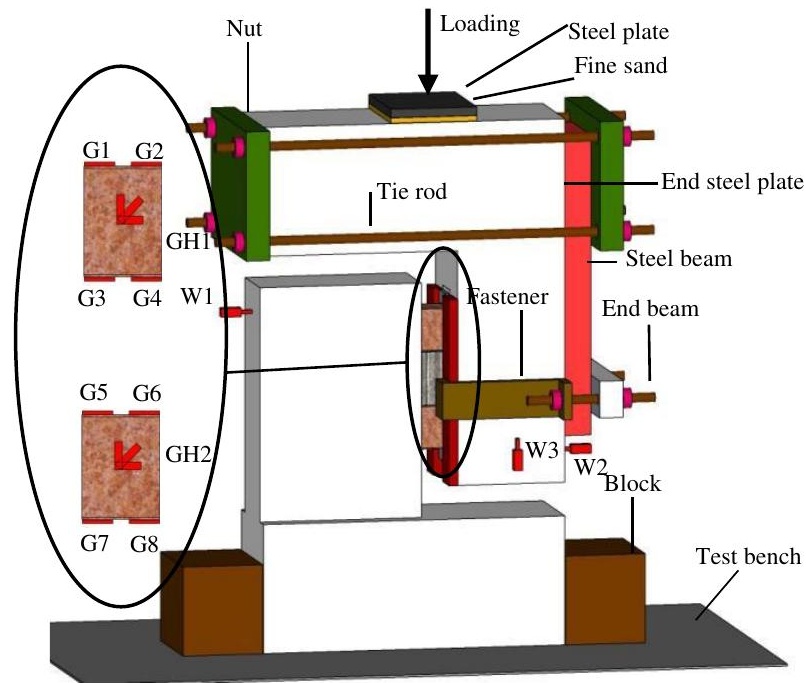

(a) Displacement gauge and strain gauge layout for specimen S4

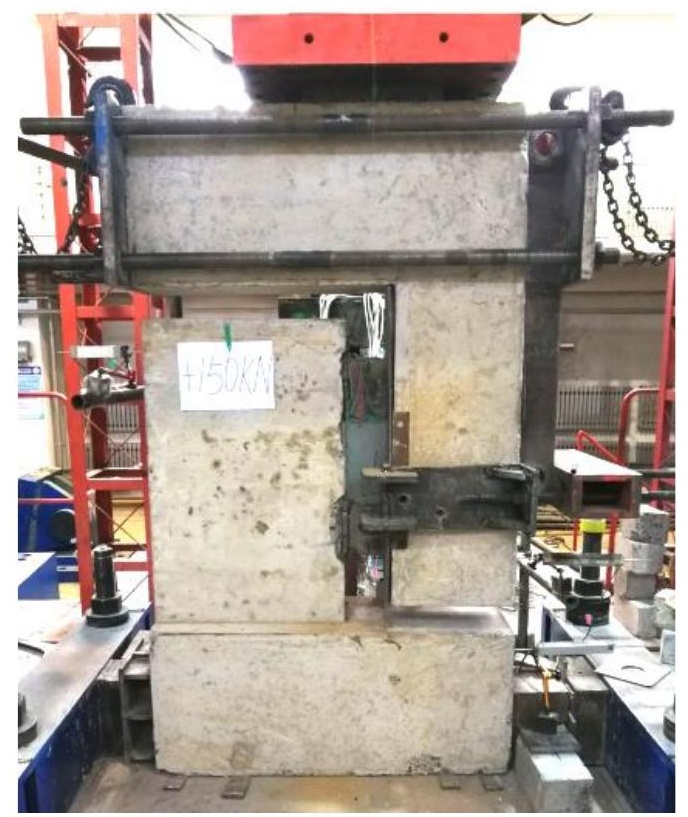

(b) SpecimenS4 and test loading device

Fig. 7 Displacement gauge and strain gauge layout for specimen S4

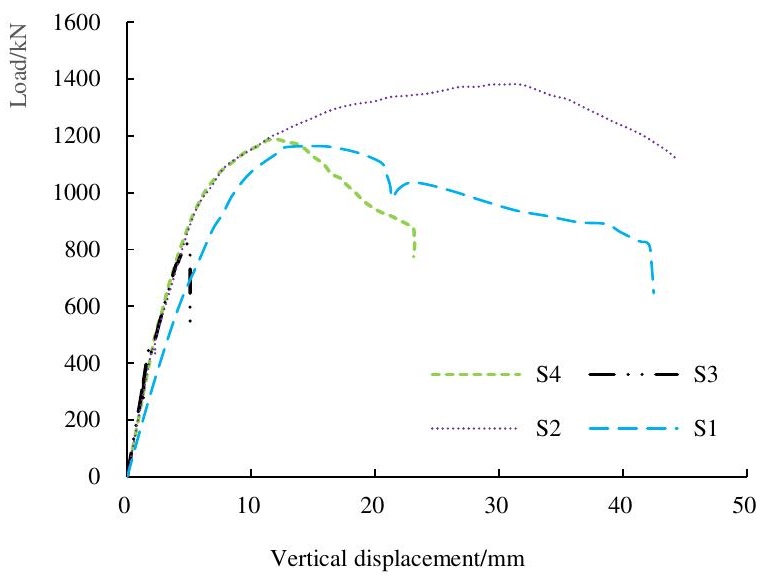

Fig. 8 Load-vertical displacement curves of each specimen 
Table 2

Material properties of each specimen

\begin{tabular}{cccccc}
\hline Specimennumber & $P_{\mathrm{y}} / \mathrm{kN}$ & $P_{\max } / \mathrm{kN}$ & $P_{\mathrm{u}} / \mathrm{kN}$ & $\mu$ & $E_{0} /(\mathrm{kN} / \mathrm{mm})$ \\
\hline S1 & 881 & 1163 & 988 & 3.8 & 169 \\
S2 & 980 & 1381 & 1173 & 6.9 & 225 \\
S3 & 666 & 822 & 698 & 1.5 & 237 \\
S4 & 910 & 1180 & 1003 & 3.09 & 207 \\
\hline
\end{tabular}

Note: $P_{\mathrm{y}}$-yield load; $P_{\max }-$ maximum load; $P_{\mathrm{u}}$-ultimate load; $\mu$ - displacement ductility coefficient; $E_{0}-$-initial stiffness

It can be seen from Fig. 8 and Table 2 that, compared with specimen S3, specimens S1 and S2 had better elasticity (before yielding load), higher elastoplasticity (between yield load and maximum load) and longer plastic section (after maximum load). Specimen S3 only showed noticeable elastic segments, while the coefficients of ductility of specimens S1 and S2 was $156 \%$ and $364 \%$ higher than that of specimen S3, respectively. In the elastic section, the initial stiffness of specimen S1 was $38 \%$ lower than that of specimen S3, and the initial stiffness of specimen S2 and specimen S3 was close. In the elastoplastic stage, the yield bearing capacities of specimens S1 and S2 were $32 \%$ and $42 \%$ higher than that of specimen S3, respectively, and their maximum bearing capacities were $42 \%$ and $68 \%$ higher, respectively. In the descending section, the ultimate bearing capacities of specimensS1 and S2 were $42 \%$ and $68 \%$ higher than that of specimen S3, respectively. The results indicate that the connection mode with and without reinforced concrete block was much better than cast-in-place structure in terms of bearing capacity and ductility. However, the initial stiffness of the latter is low. Therefore, the former has engineering feasibility.

Compared with specimen S2, specimen S4 also showed noticeable elasticity, elastoplasticity, and descending sections. Nevertheless, its descending section started earlier and its ductility coefficient was 50\% lower than that specimen S2. In the elastic section, these two almost overlapped and their levels of initial stiffness were almost identical. In the elastoplastic section, specimen S4's yield bearing capacity was $7 \%$ lower than that of specimen S2, and the maximum bearing capacity was $15 \%$ lower. In thedescending section, specimen S4's ultimate bearing capacity was $15 \%$ lower than that of specimen S2.

Overall, the results show that for the connection of the CFST keys to the column and wall, when the CFST keys was designed too strong relative to the wall, damage will be transferred to the wall, causing a decrease of the shear resistance and ductility of the overall structure. Therefore, when designing this type of connection, we should control the strength of the CFST keys.

\subsection{Analysis of the failure process of the specimen}

In order to analyze the failure process of each specimen, we took photographs of the concerned areas of each specimen at the following load: load $P=150 \mathrm{kN}$ (representing elastic section), yielding load $P_{\mathrm{y}}$, maximum load $P_{\max }$, and ultimate load $P_{\mathrm{u}}$

\subsubsection{Analysis of the failure process of specimens $S 1, S 2$, and $S 3$}

Fig.9 is a photograph of the concerned areas of specimens S1 to S3 at different load. It can be seen from the figure that for specimen S1, when yield occurred with increased load, the CFST keys did not deform significantly, and they were inclined at the maximum load. However, the steel tube itself did not show obvious buckling. At the ultimate load, the bottom steel plate at the right lower corner of the CFST keys showed obvious local buckling. As for specimen S2, the failure stage was obvious. Before the yield load, the reinforced concrete block showed no obvious oblique crack. With yield occurred with increased load, oblique cracks occurred in the reinforced concrete block, and there was no obvious local buckling in the CFST keys. During the ultimate load, the buckling deformation occurred on the surface of the CFST keys, and the through crack occurred in the oblique direction of the reinforced concrete block. For specimen S3, multiple cracks appeared when yield occurred with increased load and cracks spread over the entire concrete surface at the maximum load and the ultimate load.

It can be seen from the failure processes of specimens S2 and S1 that the reinforced concrete blocks and the CFST keys were mutually restrained. The former can effectively prevent the local buckling, and the latter can prevent the former from being damaged after a through crack happened. Therefore, with the reinforced concrete blocks placed, the initial stiffness, bearing capacity, and ductility of the overall structure were significantly improved. Meanwhile, the results also show that the overall structural failure stage of the reinforced concrete block was obvious. In the elastic stage, the reinforced concrete block and the CFST keys were intact, which can meet the seismic fortification target of "no damage under small earthquakes" After reaching the yield load, the reinforced concrete block cracked first, which reducedthe structural rigidity and enabled better ductility of the overall structure. This characteristic can help better meet the seismic fortification target of "no collapse in major earthquakes." It can be seen from the failure processes of specimens S2 and S3 that after yield occurred with increased load, specimen S3 had a faster breaking speed, and specimen S2 had slower destruction.This shows that the CFST keys connection method with reinforced concrete locks placed between the keys enhanced the seismic performance of the overall structure.

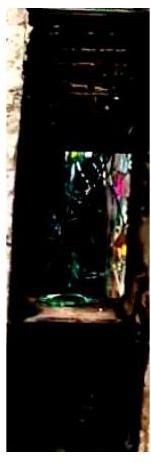

$P=150 \mathrm{kN}$

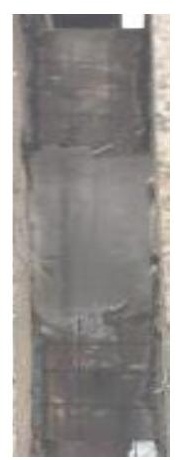

$P=150 \mathrm{kN}$

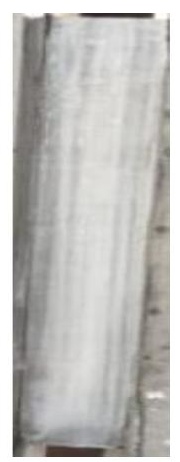

$P=150 \mathrm{kN}$

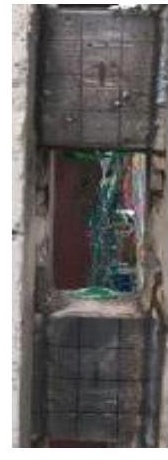

$P_{\mathrm{y}}=881 \mathrm{kN}$$$
\text { (a) Failur }
$$

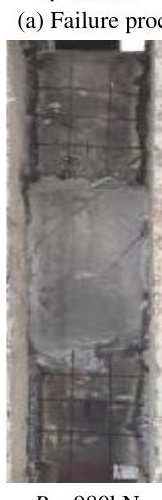

$P \mathrm{y}=980 \mathrm{kN}$

(b) Failure proces

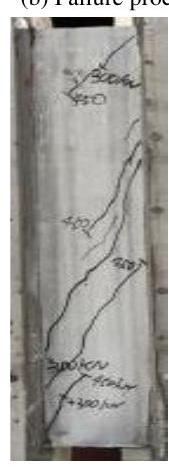

$P_{\mathrm{y}}=666 \mathrm{kN}$

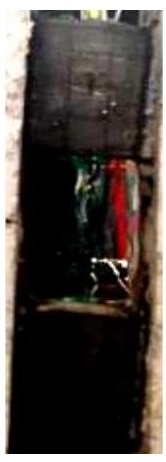

$P_{\max }=1163 \mathrm{kN}$

of specimen $\mathrm{S} 1$

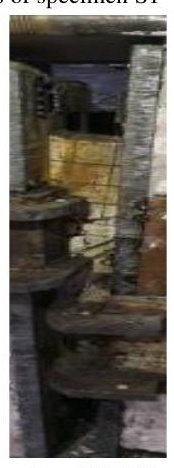

$P_{\max }=1381 \mathrm{kN}$

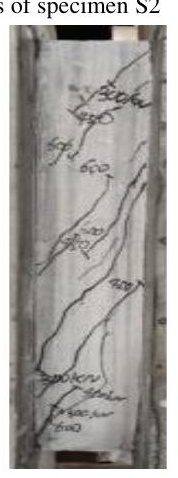

$P_{\max }=822 \mathrm{kN}$
$P_{\mathrm{u}}=988 \mathrm{kN}$

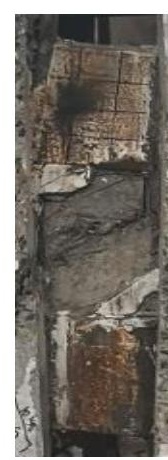

$P_{\mathrm{u}}=1173 \mathrm{kN}$

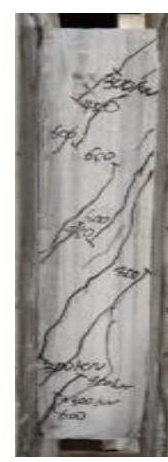

$P_{\mathrm{u}}=698 \mathrm{kN}$

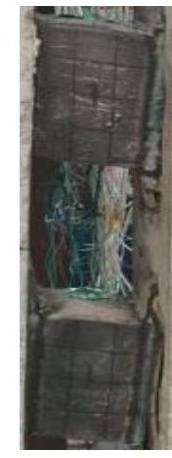

(c) Failure process of specimen $\mathrm{S} 3$

Fig. 9 Failure of the concerned areas in specimens S1 to S3 at different loading stages

\subsubsection{Analysis of the failure process of specimen $S 4$}

Fig.10 is a photograph of the concerned area of specimen S4 at different stages of loading. As can be seen from Fig.10, the failure order of the structural part of specimen S4 was the same as that of specimen S2. That is, the reinforced concrete block cracked first, and then the overall structure failed. The difference is the crack form of the two. Specimen S4 had a vertical crack first and then a diagonal crack, whereas specimen S2 had only a diagonal crack. This is mainly because the columns at both ends of the CFST keys of specimen S2 experienced a relatively larger horizontal displacement, and the force of CFST keys on the reinforced concrete block had large eccentricity.

As shown in Fig.10(c), when specimen S4 reached the maximum load, the oblique crack of the reinforced concrete block further developed and spread over the surface of the entire reinforced concrete block. A large number of the cracks were the cross-sloping ones in terms of crack form. Specimen S2 only showed a small number of oblique cracks on the reinforced concrete blocks. At the same time, it can be seen that the shape of the steel tube of specimen S4 
was hardly changed, and the degree of damage was much lower than that of specimen S2. This shows that after the CFST key was reinforced with respect to the wall, the efforts of reducing the damage of the CFST key itself would cause more serious damages to the reinforced concrete block. This is due to the fact that the deformation coordination ability of the two was poor. As shown in Fig.10(d), when the ultimate load was applied to specimen S4, the number of cracks in the reinforced concrete block and the appearance of the CFST key did not change much compared with the outcome with the maximum load, indicating that the damages at this stage were mainly on the walls.

With regard to the wall damage, under the yield load, a number of oblique cracks appeared in the upper rightpart of the wall and shear failure occurred; horizontal cracks appeared in the lower left part of the wall and bending failure occurred. As the load continued to increase to the maximum load, the number of oblique cracks and the curved cracks further increased and these cracks became longer; vertical cracks appeared in the lower right corner of the wall, and compression damage occurred. When the load decreased to the ultimate load, the reverse oblique crack appeared in the lower right corner of the wall and shear failure occurred. Meanwhile, the cracks spread over almost the entire wall and the wall was fully damaged, showing a good ductility.

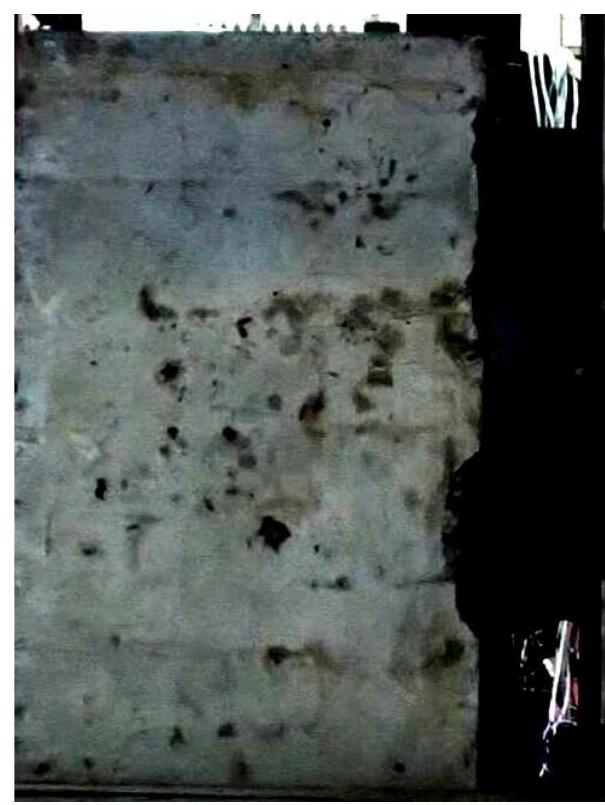

(a) $\mathrm{P}=150 \mathrm{kN}$

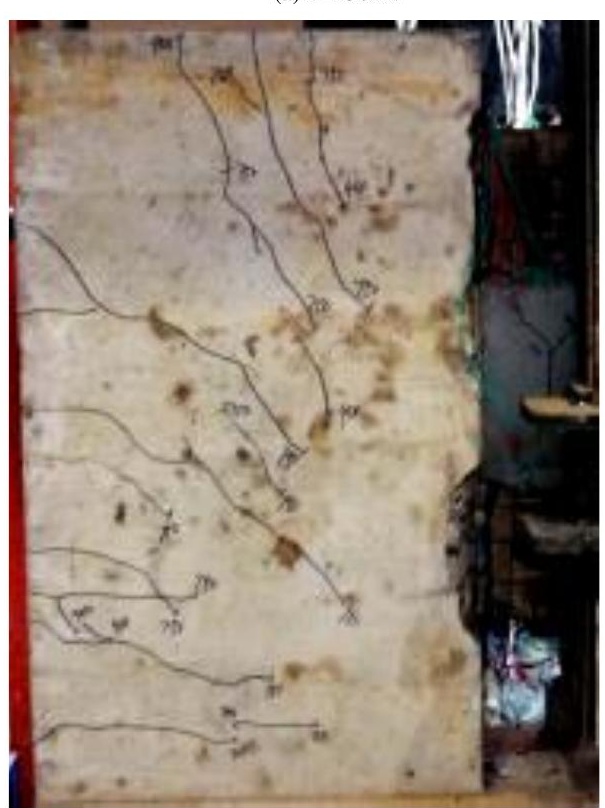

(b) $\mathrm{Py}=910 \mathrm{kN}$

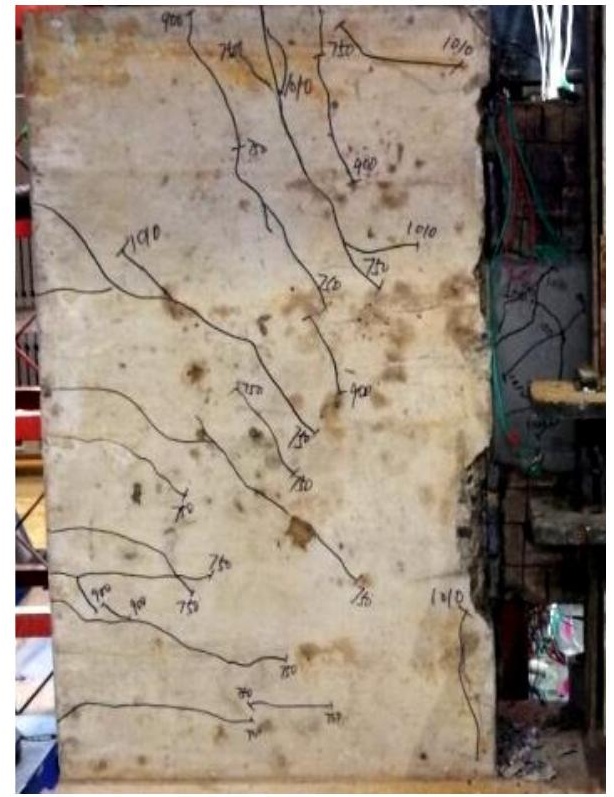

(c) $P_{\max }=1180 \mathrm{kN}$

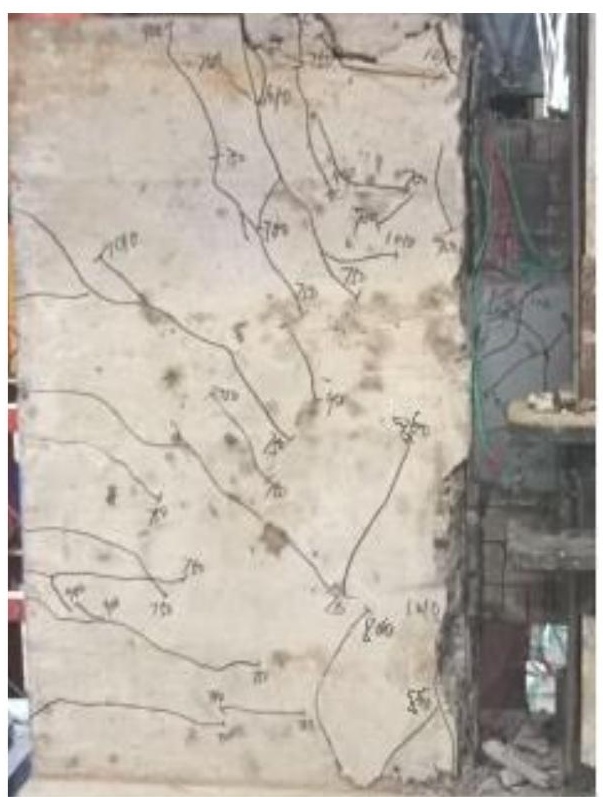

(d) $P_{\mathrm{u}}=1003 \mathrm{kN}$

Fig. 10 Photograph of the study area of specimen S4 at different loading stages

\subsection{Analysis of strain or stress of the steel tubes and steel bars}

4.3.1. Analysis of strain stress of steel tubes and steel bars of specimens $S 1$ to S3

In order to analyze the yielding condition of the steel tubes of the CFST keys, the load-strain relationship curves of strain gauges laid at the position (Y1 Y8) shown in Fig.2 for specimens S1 to S3 are extracted and shown in Fig.11. The y-axis is for the vertical load applied to the specimen and the $\mathrm{x}$-axis shows the strain measured by the strain gauge. It can be seen from Fig.11(a) and Fig.11(b) that before specimens S1 and S2 reached the yield load, the end portion of the steel tube of the CFST key had almost yielded, indicating that the strength of the steel tube is fully exerted. It can also be seen that the distribution of the strain was not completely symmetrical around the load axis, which is mainly due to the incomplete symmetry in the geometrical dimensions of the specimens, materials, and so on. It can be seen from Fig.11(c) that after the concrete reached the cracking load $(402 \mathrm{kN})$ and when the load continued to rise to about $450 \mathrm{kN}$, the steel bars yielded almost simultaneously This indicates that the shear strength of the specimens decreased sharply after the concrete cracked, and the shear enhancement effect of the steel bars was not obvious. 


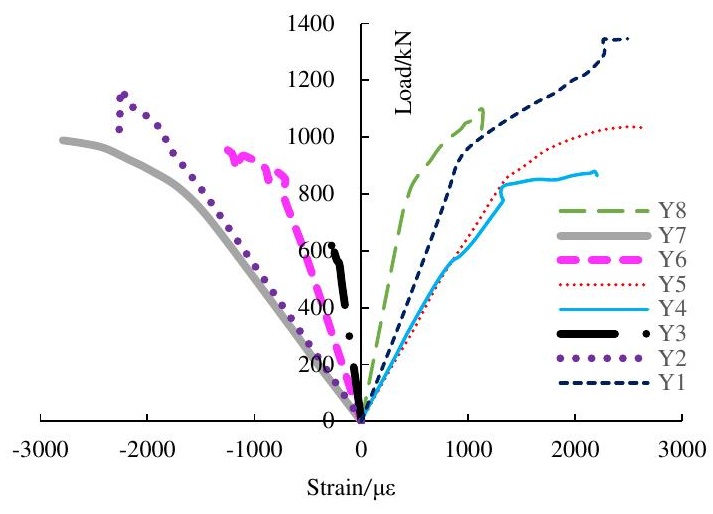

(a) Load-strain curves ofspecimen $\mathrm{S} 1$

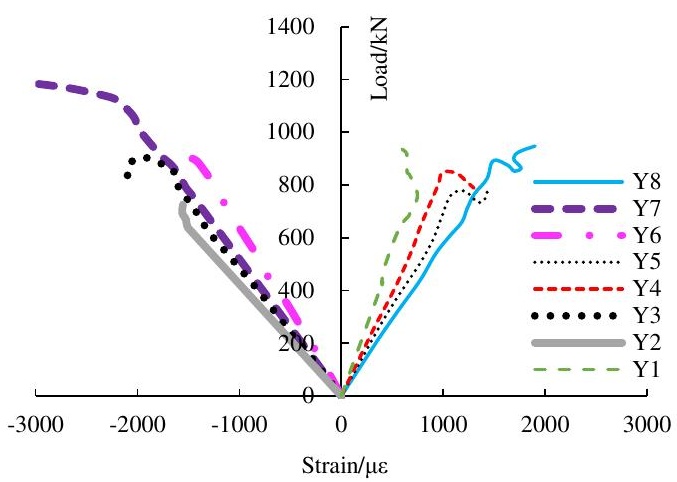

(b) Load-strain curves ofspecimen S2

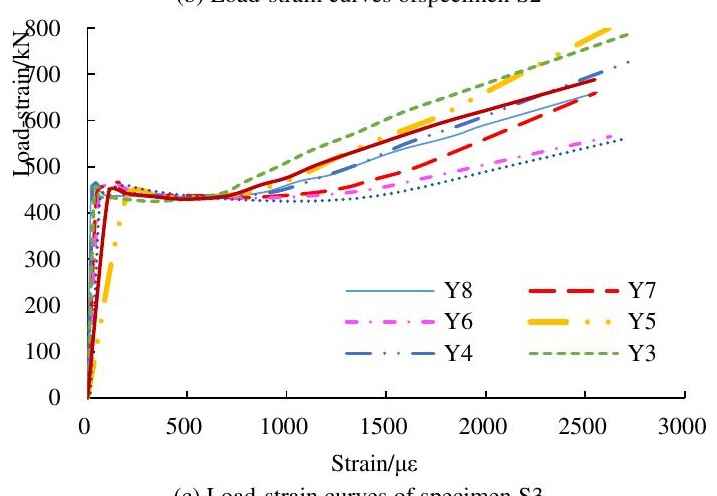

(c) Load-strain curves of specimen $\mathrm{S} 3$

Fig. 11 Load-strain curves of each specimen

To further analyze the stress state of the CFST keys, we calculated the principal stress based on the strains measured by the strain rosettes $\mathrm{H} 1$ and $\mathrm{H} 2$ on CFST keys of specimens S1 and S2. The load-principal stress curves are drawn and shown in Fig. 12. It can be seen from Fig. 12 that at the same vertical load, for both specimens S1 and S3, the principal stress at the center of the side of the upper CFST key was smaller than that of the lower one.For example, when the vertical load was $400 \mathrm{kN}$, the principal stress at the center of the upper steel tube was about $300 \%$ and $120 \%$ higher than that of the lower one for specimens S1 and S2, respectively. This indicates that the upper steel tube and the lower steel tube did not yield simultaneously under the same vertical load. This maybe because the lower part of the specimen was consolidated, while the upper part was unconstrained. Although the resultant force line of the upper load and the lower bearing resilience passed through the CFST center, the upper and lower restraints were not horizontally symmetric. When the principal stress was the same, specimen S1 had a larger vertical load difference between the upper part and lower part of the steel tube, compared with that of specimen S2. For example, when the principal stress was $200 \mathrm{kN}$, the corresponding vertical load differences between the upper and lower parts of steel tubes were $490 \mathrm{kN}$ and $250 \mathrm{kN}$ for specimens S1 and S2, respectively, which indicates that placing reinforced concrete block could make the stress of the upper and lower CFST keys more evenly.

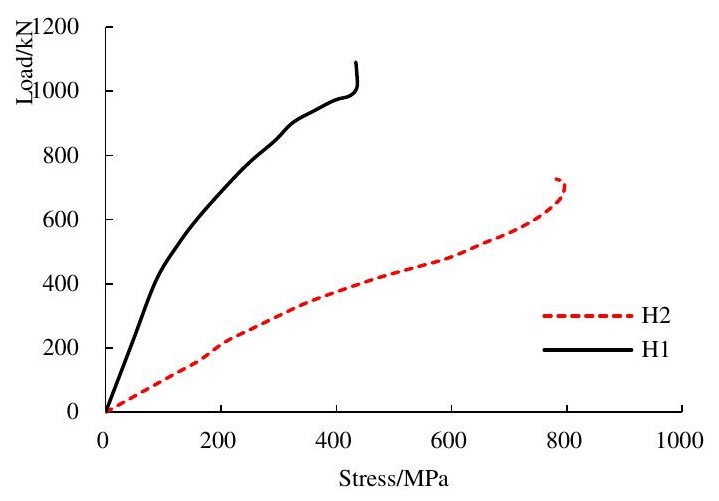

(a) Principal stress calculated from strain gauge rosettes on specimen S1

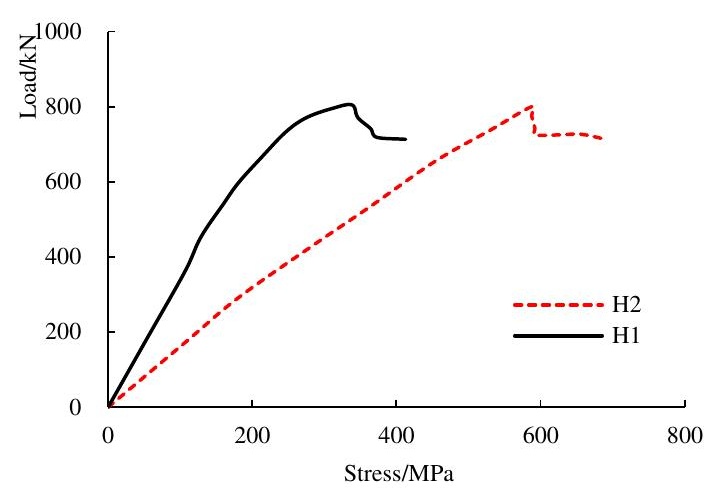

(b) Principal stress calculated from strain gauge rosettes on specimen S1

Fig. 12 Principal stress calculated from strain gauge rosettes on specimens S1 and S2

\subsubsection{Analysis of strain or stress of the steel tubes and steel bars of specimen} S4

(1) Analysis of strain of steel bars.

Because some of the steel strain gauges were damaged during the production of the specimen, only two sets of horizontal strain gauges and two sets of vertical strain gauges were selected to analyze the force behavior of the steel bars in the horizontal and vertical directions. The load-strain curves are shown in Fig. 13.

Fig.13(a) and Fig.13(b) are the curves of strain and vertical load as measured by strain gauges S1, S2, S3, S7, S8, and S9 which are shown in Fig.3. As can be seen from these two figures, for the same horizontal steel bars, the ones that were close to one end of the CFST keys (S1 and S7, referred to as the proximal end) received the stress lastly; the middle part of the steel bar (S2 and S8) was the first to be stressed; and the steel bar that was away from the end of CFST keys (S3 and S9, referred to as the distal end) was stressed next. The middle and distal end of the steel bar yielded while the proximal end did not yield. This result shows that when shear was transmitted through the CFST keys in the horizontal direction, the middle part of the steel bar was firstly stressed, then the distal end, and finally the proximal end, which is consistent with the development of the cracks shown in Fig. 10.

Fig.13 (c) and Fig.13 (d) are the curves of strain and vertical load measured by strain gauges S1, S10, S16, S2, S8, and S17 which are shown in Fig.3. As can be seen from Fig.13 (c), for the steel bars placed in the vertical direction, the lower part of the steel bars (S16) was stressed firstly, then, the middle part (S10), and lastly, the upper part (S1). Only the area measured by S16 exceeded the yield strain. Fig.13 (d) shows that the loading process of the steel bar was opposite to that illustrated in Fig.13 (c). The upper part of the steel bar (S2) was stressed firstly, then, the middle part (S8), and lastly, the lower part (S17) is the last. Even the area measured by S17 showed some effect of compressive strain. These phenomena are also consistent with the crack development on the walls shown in Fig.10(c) and Fig.10(d), in which the cracks were distributed in the middle and upper parts, and the oblique cracks appeared in the lower right part when the ultimate load was reached. These results show how the CFST keys transmitted shear in the vertical direction. The steel bar that was on the lower side of the CFST keys was pressed first. When the steel bar was away from the CFST keys, its upper part was stressed first. 


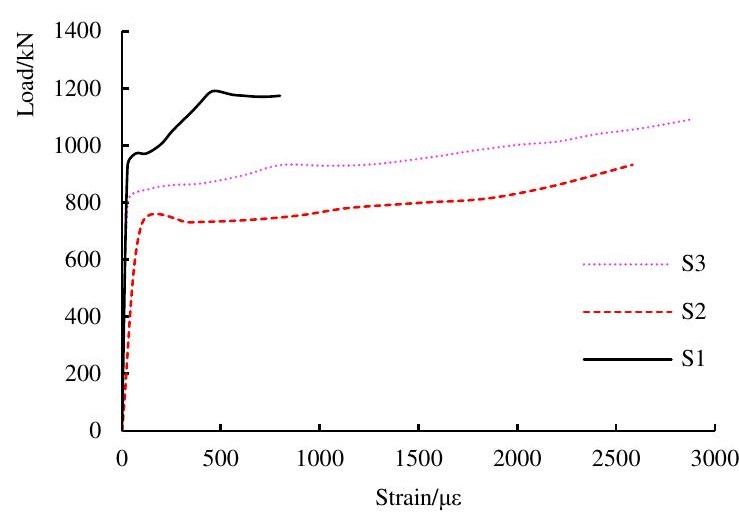

(a) Load-strain relationship curves measured by $\mathrm{S} 1, \mathrm{~S} 2$, and S3

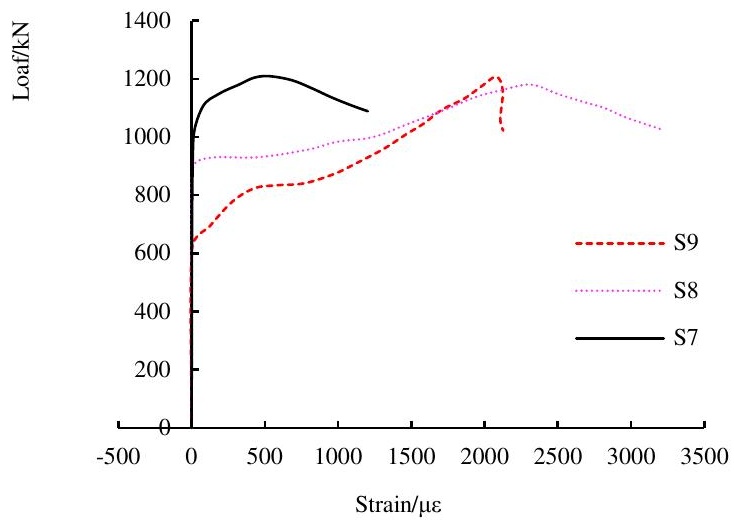

(b)Load-strain relationship curves measured by S7, S8 and S9

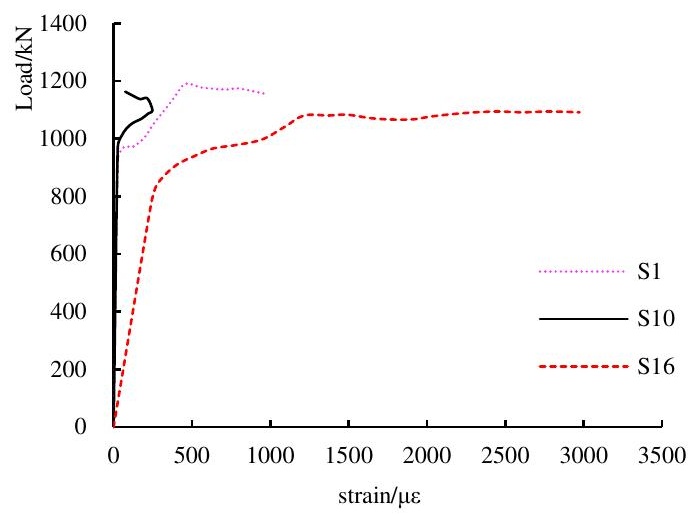

(c) Load-strain relationship curves measured by S1, S10, and S16

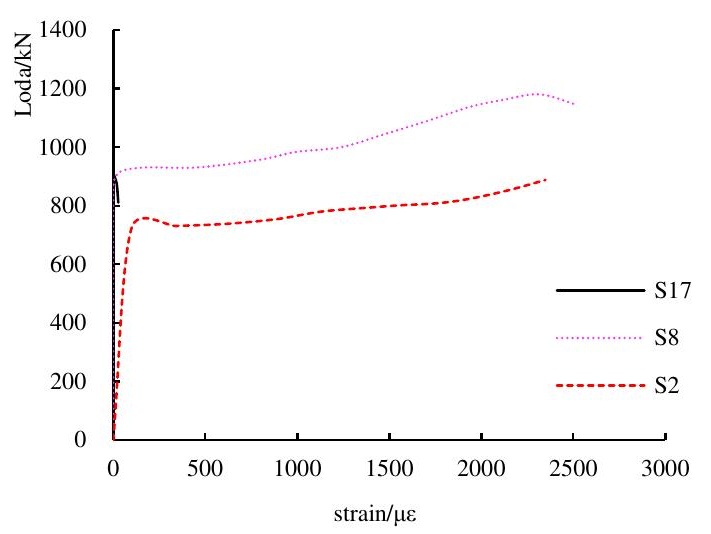

(d) Load-strain relationship curves measured by S2, S8, and S17

Fig. 13 Load-strain relationship curves of the steel bars

(2) Stress and strain analysis of the steel tubes

Fig.14 shows load-strain relationship curves of specimen S4 at the test areas as specified in Fig.7. It can be observed that the strain of the steel tube was less than 800 , indicating that after the steel tube was strengthened, the upper surface steel place and the lower surface steel plate of the CFST keys were not yielded. Fig.15 shows load-principle stress relationship curves of specimen S4 in the center of the side steel plate of CFST keys. The principle stress is calculated from the strain measured by GH1 and GH2. It can be seen that the stress at the center of the side steel plate of CFST keys exceeded the yield stress. This shows that although the steel plate of the CFST keys was reinforced, the center part of the side steel plate was still yielded.

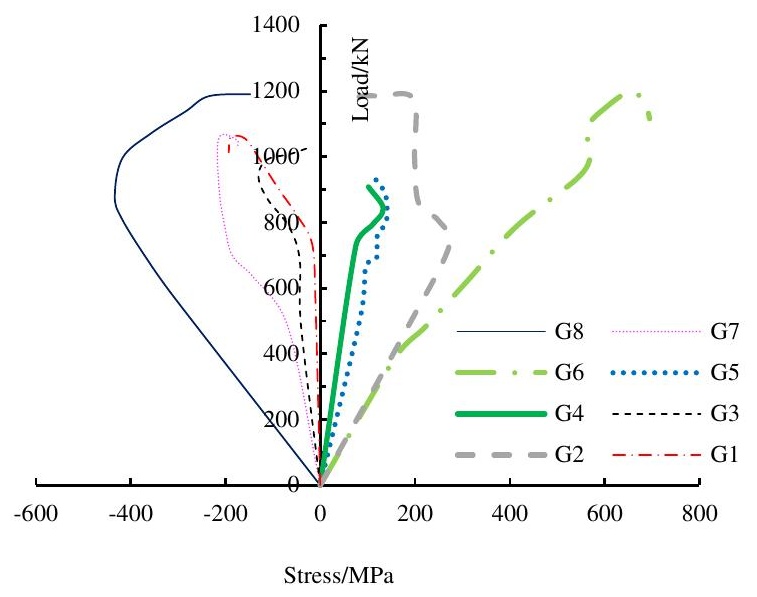

Fig. 14 Load-strain curves of the steel tube of specimen S4

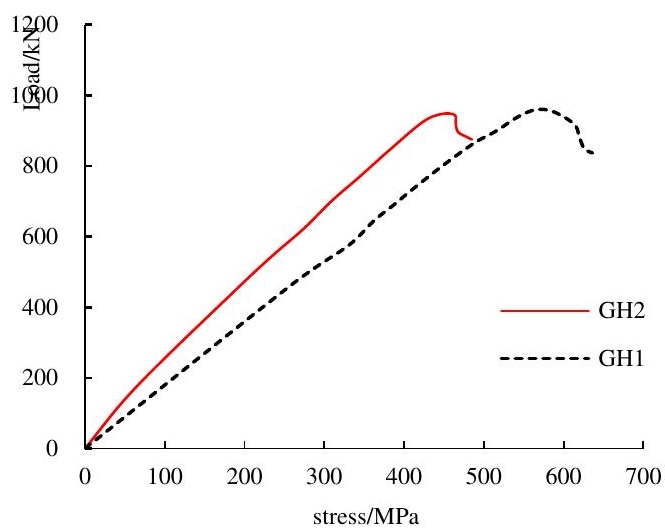

Fig. 15 Load-stress curves of the steel tube of specimen S4

\section{Analysis of Horizontal displacement between wall and column}

Fig.16 shows a vertical load-horizontal displacement curves of specimens $\mathrm{S} 1$ to $\mathrm{S} 4$, where the horizontal displacement was measured by displacement gauges W1 and W2 whose positions are shown in Fig.4(b), Fig.4(c), and Fig.7(a). It can be seen from Fig.16 that the displacements measured by W1 and W2 were small for the specimens and the maximum values were less than $9 \mathrm{~mm}$. These results indicate that the test device basically satisfied the requirement of vertical symmetry.

The displacements measured by W2 for the specimen were larger than those measured by W1 with smaller differences at the elastic stages and relatively larger differences at the inelastic stages. Specimens S3 had the smallest difference, with a maximum difference less than $0.6 \mathrm{~mm}$. The differences between the specimens that were connected by the CFST keys were relatively larger, and the maximum difference was $7.09 \mathrm{~mm}$. This shows that for the walls and columns connected by the CFST keys, when the shear was applied, the relative displacement between the walls and columns may be relatively large in the horizontal direction. This relatively large deviation is mainly due to the large inelastic deformation occurring in the inelastic stage of the CFST keys, which breaks the symmetry. 


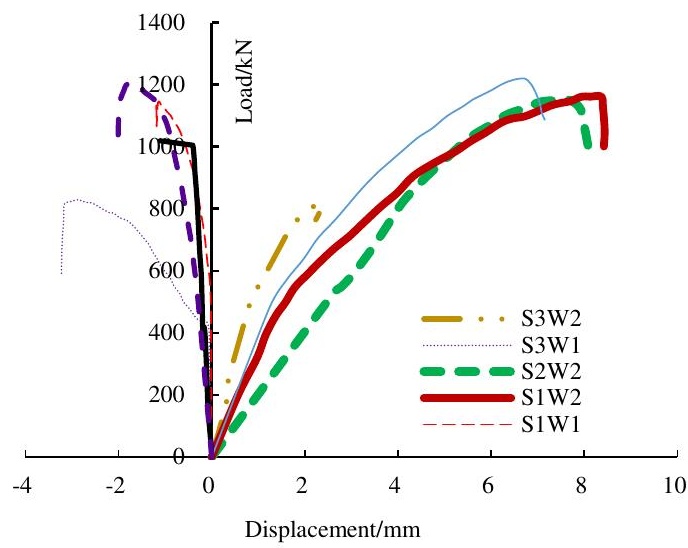

Fig. 16 Load-horizontal displacement curves measured by displacement meter W1 and W2

\section{Simplified calculation formula for shear bearing capacity}

The total shear bearing capacity of the CFST keys with the reinforced concrete block between them is composed of two parts: One is the shear capacity of the CFST keys, and the other is the compressive bearing capacity of the reinforced concrete block. Regarding the shear capacity of CFST keys, the calculation formulas adopted by the Chinese and American codes are simple, that is, only considering the shear strength of steel tubes and ignoring the role of concrete (ANSI/AISC 360-05[32], CECS 159:2004[33]). Considering that the damage form of the reinforced concrete block before the overall structure failure is close to the damage form of the member under axial compression, we adopted the calculation formula of the axial bearing capacity as a simplified formula to calculate the bearing capacity of the reinforced concrete blocks.Therefore, the simplified calculation formulacan be obtained as follows:

\section{$V=2 n \cdot t_{s} \cdot h_{s} \cdot f_{v}+(n-1) f_{c} b_{c} h_{c}(1)$}

Where $V$ is thetotal shear bearing capacity of the CFST keys with the reinforced concrete block between them; $n$ is the number of CFST keys; $t_{\mathrm{s}}$ and $h_{\mathrm{s}}$ are the thickness and height of rectangular steel tube, respectively; $f_{\mathrm{v}}$ is the shear yield strength of steel; $f_{\mathrm{c}}, b_{\mathrm{c}}$, and $h_{\mathrm{c}}$ are the compressive strength, section width, and section height of the reinforced concrete block, respectively.

The shear capacity of specimens S2 and S4 were calculated to be $993 \mathrm{kN}$ and $1507 \mathrm{kN}$, respectively, which were $1.3 \%$ and $65 \%$ higher than the yield load of specimen S2 and specimen S4, respectively. The calculated results were in line with that for specimen S2; however, the calculated results deviated greatly from that for specimen S4. This is mainly because the equation assumes that the steel plate section all meets the yield strength $f_{\mathrm{v}}$ of the shear key, while specimen S4 had seen wall damage mostly and only a small part of the steel plate reached the yield strength. This shows that the equation can be used to estimate the shear capacity of the connection.

\section{Conclusions}

This paper describes the shear test of the CFST keysconnected shear wall and columns in the frame-shear wall structure and presents the test results systematically.A practical method for wall and column connection isproposed ,which shows good mechanical properties. We also established the simplified calculation formulaforthetotal shear bearing capacity ofthis connection.These works provide a powerful basis for exploring the application of composite structural members to prefabricated concrete structures. The main findings of this study are as follows:

(1)With regard to the wall and column connection by the CFST keys with reinforced concrete blocks placed between them, its bearing capacity and ductility were better than the cast-in-place structure, while the initial stiffness was the same as the cast-in-place structure.It shows good engineering feasibility.

(2)The wall-column connection method of the CFST keys with reinforced concrete blocks showed good seismic performance. In the elastic stage, the reinforced concrete blocks were intact and the structural rigidity was almost equal to the cast-in-place structure. This connection can meet the seismic fortification target of "no damage under small earthquakes." After yielding, the reinforced concrete blocks were damaged, and the structural rigidity decreased. The CFST keys and reinforced concrete blocks exerted constraints on each other, enabling a high bearing capacity and ductility. These characteristics can help meet the seismic fortification target of "no collapse in major earthquakes."

(3) When designing the connection of wall and column by the CFST keys with reinforced concrete blocks placed between them, we should control the strength of the CFST keys properly. When the CFST keys are too strong in design, the damage to the reinforced concrete block will increase and the structural damage will be transmitted to the wall, leading to the reduction on bearing capacity of the whole structure.

\section{Acknowledgement}

This project was completed with the support of the Thirteenth National Key-point Research and Invention Program (2017YFC0703805) and Scientific Research Fund Project of Liaoning Provincial Department of Education(LNQN201907).

\section{References}

[1] Yuan S.M., "Experimental Research on Frame-shear Wall Structure Based on differen Column-Wall Connection Ways ", Master's Thesis, Shenyang Jianzhu University,Shenyang, China, 2016.

[2] Si X.B., "Research on the shear performance of prefabricated connection", Master's Thesis, Shenyang Jianzhu University,Shenyang, China, 2017.

[3] Restrepo J.I., Park R., and Buchanan A.H., "Tests on connections of earthquake resisting precast reinforced concrete perimeter frames of buildings", PCI Journal, 40(4), 44-61, 1995.

[4] Li N., Zhang J.C., CHU X.F. and LIU B., "Experimental study on seismic behavior of pre-cast concrete beam-column sub-assemblage with cast-in-situ monolithic joint", Engineering Mechanics, 26(S1), 41-44, 2009.

[5] Li S.D., "Experimental study on seismic performance of precast concrete columns and joints", Master's Thesis, Harbin Institute of Technology, Harbin, China, 2013.

[6] Alcocer S.M., Carranza R., Perez-Navarrete D.,et al., "Seismic tests of Beam-to-Column Connections in a precast concrete frame", PCI Journal, 47(3), 70-89, 2002.

[7] Guan D.Z., Guo Z.X., Yu J.B., et al., "Structural measures and experimental study of precas concrete joints of anchored prestressing strands in frames" Journal of Zhejiang University(Engineering Science), 50(2), 282-291, 2016.

[8] Zhao Y., Li Y.B., Bi Q., et al., "Experimental investigation on seismic performances of knee joints for monolithic precast concrete frame", Journal of Tongji University(Natural Science), $47(5), 600-608,2019$.

[9] Lu X.L., Wang L., Wang D., et al., "An innovative joint connecting beam for precast concrete shear wall structures", Structural Concrete, 17(6), 972-986, 2016.

[10] Chen J.W. and Su Y.P., "Prefabricated concrete shear wall structure and its connecting technology", World Earthquake Engineering, 29(1), 38-48, 2013.

[11] Sun J.,QiuH.X.,Lu Y., et al., "Experimental study of lateral load behavior of H-shaped precast reinforced concrete shear walls with bolted steel connections", The Structural Design of Tall and Special Buildings, 2019, https://doi.org/10.1002/tal.1663.

[12] Song G. and Wang Y., "Low frequency cyclic loading tests of L-shaped precast sandwich insulation shear wall", Building Structure, 46(10), 20-23, 2016

[13] Lu Z., Wang Y., Li J.B., et al., "Experimental study on seismic performance of L-shaped insulated concrete sandwich shear wall with a horizontal seam", The Structural Design of Tall and Special Buildings, 28(1), 2019.

[14] JGJ101-96, Specification of testing methods for earthquake resistant building, Ministry of Housing and Urban-Rural Development of the People's Republic of China, Beijing, 1996.

[15] Smith B.J., Kurama Y. C., and McGinnis J, "Behavior of Precast Concrete Shear Walls for Seismic Regions: Comparison of Hybrid and Emulative Specimens", Journal of Structural Engineering, 139(11), 1917-1927, 2013.

[16] Pekau O. A. and Hum D., "Seismic response of friction jointed precast panel shear walls", PCI Journal, 36(2), 56-71, 1991.

[17] Soudki K. A., Rizkalla S. H. and LeBlanc B., "Horizontal connections for precast concrete shear walls subjected to cyclic deformations part 1: Mild steel connections", PCI Journal, 40(4), 78-96, 1995.

[18] Li J.B., Fan Q.Q., Lu Z., et al., "Experimental study on seismic performance of T-shaped partly precast reinforced concrete shear wall with grouting sleeves", Struct Design Tall Spec Build, e1632, 2019, https://doi.org/10.1002/tal.1632.

[19] Soudki K. A., Rizkalla S. H., and Daikiw R. W., " Horizontal connections for precast concrete shear walls subjected to cyclic deformations part 2: Prestressed connections", PCI Journal, 40(5), 82-96, 1995

[20] Wang Z., "Study on Mechanical Properties and Design Methods of the Equivalent Replacement Rebar Pre-Cast Concrete Coupling Beams", Master's Thesis, Shenyang Jianzhu University,Shenyang, China, 2016

[21] Gao J., "Study on performance of concrete filled steel tubular composite keyway connection in assembled frame-shear structures",Master's Thesis, Shenyang Jianzhu University,Shenyang, China, 2019.

[22] Liang Q.C., "Study on mechanical properties and design method of steel-connected concrete Beams", Master's Thesis, Shenyang Jianzhu University,Shenyang, China, 2019.

[23] Mo C., "Study on seismic performance of fully precast frame-shear wall structure", Master' s Thesis, Southeast University, Nanjing, China, 2017.

[24] Ma J.W., "Study on seismic behavior of the new full precast concrete shear-wall frame stru ctures", Ph.D. Dissertation, Southeast University, Nanjing, China, 2018

[25] Wu D.Y., LIANG S.T., GUO Z.X., et al., "Experimental study on grouted connecting precast shear-wall spatial structural model", Journal of Building Structures, DOI: 10.1 4006/j.jzjgxb.2018.0426.

[26] Wu Y.H., YUE Q.R., ZENG B., et al., "Research on seismic design of prefabricated e nclosure wall for industrial buildings", Industrial Construction, 47 (4) , 166-173, 2017.

[27] Bin L S and LEI D., "The development of prefabricated concrete structures", 20142 n d International Conference on Mechanical Structures and Smart Materials, Malaysia, 81 $-84,2014$.

[28] Ming L. and YANG Z. H., "The Connection Form of The Beam and Column in Prefa bricated Concrete Structure", 4th International Conference on Civil Engineering,Archite cture and Building Materials(CEABM2014), Haikou, 818-821, 2014. 
[29] GB50017-2017, Standard for design of steel structures, Ministry of Construction of Ch ina, Beijing, China, 2017.

[30] GB50010-2010, Code for design of concrete structures, Ministry of Construction of Ch ina, Beijing, China, 2010.

[31] GB /T 50152-2012, Standard for test method of concrete structures, Ministry of Construction of China, Beijing, China, 2012

[32] Li Z.H., PENG Z.H., QI Y.H., et al., "Full-scale experimental study on seismic behaviors of plasticity controllable steel joint of prefabricated RC beam column", Journal of Building Structures, 40(10), 43-50, 2019.

[33] ANSI/AISC 360-05, Specification for Structural Steel Buildings, American Institute of Construction, Chicago, USA, 2005.

[34] CECS 159:2004, Technical Specification for Structures with Concrete-filled Rectangular Steel Tube Members, China Association for Engineering Construction Standardization, Shanghai, China, 2004. 


\title{
EXPERIMENTAL AND THEORETICAL STUDY ON THE BEHAVIOR OF THE LAMINATED ACTION OF STEEL-CONCRETE COMPOSITE BEAM IN NEGATIVE BENDING MOMENT REGION
}

\author{
Xian Liang, Shu-Jin Duan *, Yuan-Yuan Wang and Yan-Qing Zhang \\ Research Institute of Structural Engineering, Shijiazhuang Tiedao University, Shijiazhuang, China \\ *(Corresponding author: E-mail: duanshujin@stdu.edu.cn)
}

\section{A B S T R A C T}

A new type of steel-concrete composite-laminated action beams (CLB) is developed to improve the crack resistance of top concrete slabs. It is an improved version of double steel-concrete composite beams (DCB), in which the shear stress between top concrete slab and upper steel flange is released by the uplift-restricted and slip-permitted connectors (URSP). To investigate the static mechanical behavior of CLBs in the negative moment region, an experimental test on two CLB specimens and one DCB specimen was carried out, and the theoretical study on the distribution equation of interface slip, the calculation formulas of sectional bending stiffness and ultimate bending moments was conducted. The results show that although the flexural bearing capacity of CLB is slightly lower than that of DCB, the crack resistance was markedly better than that of DCB. The slips and ultimate bending moments predicted with the simplified formulas are good in agreement with tests results.

\section{A R T I C LE H I S T O RY}

$\begin{array}{ll}\text { Received: } & \text { 25 September } 2019 \\ \text { Revised: } & \text { 4 May } 2020 \\ \text { Accepted: } & \text { 30 May } 2020\end{array}$

\section{KE YWO R D S}

Composite-laminated action beam; Cracking resistance;

Slippage; Bearing capacity;

Double composite beam;

Experimental study

Copyright $\odot 2020$ by The Hong Kong Institute of Steel Construction. All rights reserved.

\section{Introduction}

Traditional steel and concrete composite beams have been widely used in civil engineering in the past several decades because of their low weight, low cost, and the performances of the full use of the two materials. However, in the negative bending moment region of continuous beams, especially the long-span continuous beams, concrete cracking and steel flange buckling always troubled engineers [1-3]. In order to alleviate the local yield of the pressed web and the bottom flange of the steel beam, the double composite beam (DCB) was proposed by Reiner [4], as shown in Fig. 1 (a). In DCBs, the bending stiffness and strength were enhanced and local buckling of the steel profile in the negative moment region was prevented by attaching a concrete slab to the bottom flange of the steel beam.

In order to study the behavior of DCB, extensive researches has been conducted. Steven et al. [5] introduced that the inclusion of the double composite action provided several beneficial features: steel consumption was saved, a "braced effect" let the steel stress reduction factor unnecessary; a compact section is reasonably achieved in the negative flexural region, and the redistribution of moments becomes favorable; the width of frame may be reduced due to the bracing of bottom flange of the girder by bottom slab. Experiment studys [6]showed that the DCB could prevent local buckling of steel profile, improve the ductility of structure, and enlarge crack widths but slow crack development to some extent. Duan et al. [7-10] investigated the mechanical properties of DCB in the negative moment region by conducting experimental, numerical and theoretical studies. It was concluded that the elastic ultimate load of the DCB was increased about $50 \%$ compared the traditional composite beam, and under the action of the same load, the addition of lower concrete slab greatly reduced the maximum crack width.

In the DCB, the bending stiffness of cross sections was greatly increased, and the steel beam yielding under compression was alleviated. However, the neutral axis of cross sections was moved down, so the cracking in the top concrete slab was severe still $[6,8]$.

Concrete cracks will degrade the cross-section stiffness and reduce the suitability and durability of structures. To improve the crack resistance of top concrete slab effectively in the negative moment region, many investigations have been conducted. Experimental studies [11] found that cracks concentrated at the cast interface of joints between decks even in the negative moment region. The inelastic behavior of the negative moment region and the cracking characteristics of the composite beams were investigated in the reference [12-13]. Researchers began to look for new design methods, materials and techniques. The crack development pattern was summarized, and a formula for calculating the maximum crack width was also given [14-18].

In practical engineering, the application of prestressed is the most common and effective method to relieve the cracking of concrete slab [19-20]. However, the prestressed construction process is complicated; the efficiency is low; and the bending stiffness improvement in the negative bending moment region is very limited. Therefore, scholars began to seek new ways to relieve the concrete cracking. The variation of strength and stiffness properties of beams with various degrees of composite beam was investigated in the literature [21], and the influence of the degree of partial composite action on flexural behavior was assessed. Experimental studies [22] showed that the cracking of the top concrete slab could be relieved by reducing the number of shear connectors. Changing the arrangement of the shear connectors, the cracking bending moment of the top concrete slab can be improved, and the maximum width of cracks also can be reduced [23]. Based on the viewpoint of reducing the transmission of the shear stress while maintaining the consistent of the vertical displacement of the concrete slab and the steel beam, the uplift-restricted and slip-permitted (URSP) connector was proposed [24]. And the mechanical properties and slip performance of URSP connectors have been investigated by conducting experiment and theoretical analysis [25-26].

With the DCB and URSP connectors as the background, a composite-laminated action beam (CLB) applied in the negative bending moment region of continuous composite beams was proposed [27]. The construction of CLB is shown in Fig. 1(b), in which the connectors connecting the top concrete slab and the steel beam is URSP connectors. According to the research about CLBs applied in the practical engineering, the composite-laminated action can effectively improve the stiffness of the cross-section in the negative bending moment region of continuous composite beams, and adjust the internal force of structures, thereby improving the mechanical performance of structures [28-29].

In order to study the mechanical properties of the new structure - CLB, an experiment of negative moment region was conducted. Two CLB specimens and one DCB specimen with the same parameters were designed and tested for comparison. The theoretically study of CLB is also conducted. The formulas for calculating the ultimate flexural capacity, bending stiffness, and slip distribution of CLB were proposed.

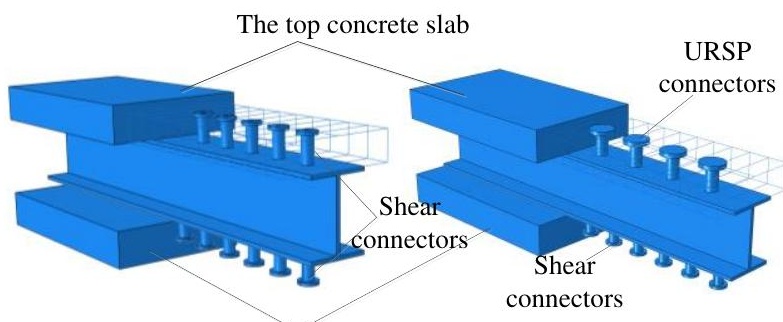

The bottom concrete slab

(a) DCB

(b) CLB

Fig. 1 Steel-concrete composite beam with different composition 


\section{Experimental studies}

\subsection{Specimens}

As stated above, two CLBs (CLB1 and CLB2) and one DCB specimens were manufactured and tested. All these specimens had the same dimensions. Each specimen was $3.2 \mathrm{~m}$ long, and the net-span was $3.0 \mathrm{~m}$ with a $100-\mathrm{mm}$ extent portion at each edge support. The main characteristics and nominal dimensions of test specimens are shown in Fig. 2(a), (b) and (c).

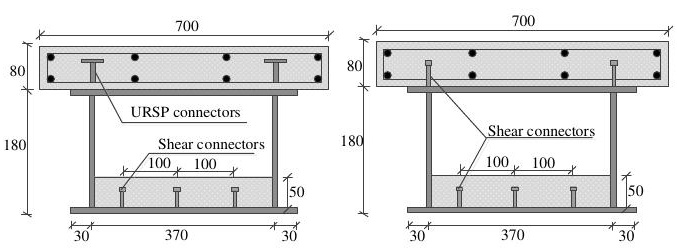

$\begin{array}{ll}\text { (a) The cross section of CLB } & \text { (b) The cross section of DCB }\end{array}$

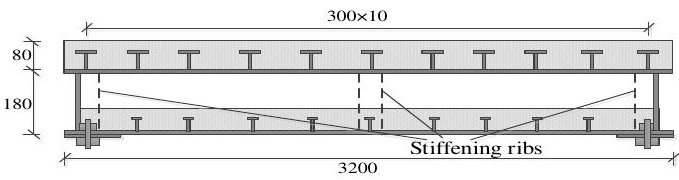

(c) The arrangement of connectors

Fig. 2 Parameters of test beams (mm)

In the CLB, a laminated interface was formed between the top concrete slab and the steel beam using the URSP connectors, so the cross section was divided into top and bottom beams along the laminated interface.

The top concrete slab was $80 \mathrm{~mm}$ thick and $700 \mathrm{~mm}$ wide, and the bottom concrete slab was $50 \mathrm{~mm}$ thick and $362 \mathrm{~mm}$ wide. In the top concrete slab, 8 longitudinal rebars with $12 \mathrm{~mm}$ diameter in two layers, and transverse bars with $6 \mathrm{~mm}$ diameter and $100 \mathrm{~mm}$ spacing were reinforced.

The steel beam was welded by 8-mm-thick SS400 steel plates. It was 180 $\mathrm{mm}$ high and $430 \mathrm{~mm}$ wide. Stiffening ribs were established at the support and loading sections, as shown in Fig. 2. The diameter and height of the connectors were $13 \mathrm{~mm}$ and $50 \mathrm{~mm}$ respectively. Fig. 3 shows the construction of the URSP and shear connectors.

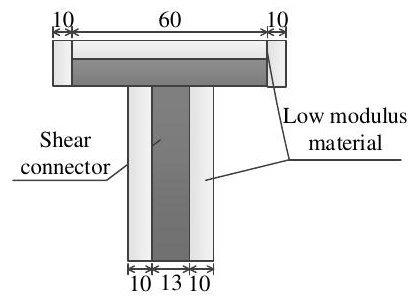

(a)URSP connector

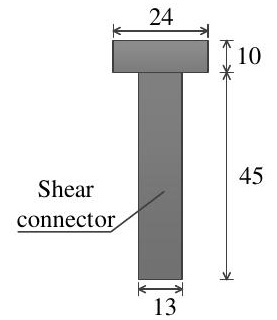

(b)Shear connector
Fig. 3 The Construction of connectors

\subsection{Material Properties}

The concrete material properties of specimens were tested on test day. The uniaxial compressive and tensile strengths were obtained based on $150-\mathrm{mm}^{3}$ cubic specimens. The mean yield strength, ultimate strength, and Young's modulus of welded steel plates and reinforcing steel bars were tested. The results are given in Table 1.

\subsection{Test Setup and Loading Instrumentation}

To simulate the stress state in the negative moment region, two ends of each specimen were connected to the ground though military piers. A reverse vertical load was applied to the mid-span by a hydraulic jack. The setup and test details are shown in Fig. 4.

In the negative moment region, as shown in Fig. 5, strain gauges were mounted on several critical cross sections of specimens and steel reinforcing bars to derive the sectional strain distribution. These critical cross sections were mainly positioned at $1 / 4$ and $3 / 4$ of the span, as well as at the cross sections 200 $\mathrm{mm}$ and $400 \mathrm{~mm}$ away from the mid-span. Fig.5 demonstrates the distribution of strain gauges mounted in the cross section of the 1/4 span, and strain gauges in other critical cross sections were mounted in this manner.

Table 1

Mechanical properties of materials $(\mathrm{MPa})$

\begin{tabular}{|c|c|c|c|c|c|c|c|}
\hline Concrete & $f_{\mathrm{c}}$ & $f_{\mathrm{t}}$ & $E_{c}$ & Steel & $f_{\mathrm{y}}$ & $f_{\mathrm{u}}$ & $E_{\mathrm{s}}$ \\
\hline \multirow{2}{*}{ Top } & \multirow{3}{*}{27.8} & \multirow{3}{*}{3.12} & \multirow{3}{*}{$3.25 \times 10^{4}$} & Steel plate & 378.1 & 484.5 & $2 \times 10^{5}$ \\
\hline & & & & Steel & \multirow{3}{*}{456.7} & \multirow{3}{*}{615.0} & \multirow{3}{*}{$2.06 \times 10^{5}$} \\
\hline \multirow{2}{*}{ concrete } & & & & reinforce & & & \\
\hline & \multirow{4}{*}{29.0} & \multirow{4}{*}{3.20} & \multirow{4}{*}{$3.25 \times 10^{4}$} & bar $\Phi 12$ & & & \\
\hline \multirow{3}{*}{$\begin{array}{l}\text { Bottom } \\
\text { concrete }\end{array}$} & & & & Steel & & & \\
\hline & & & & reinforce & 300.5 & 420.2 & $2.06 \times 10^{5}$ \\
\hline & & & & bar $\Phi 6$ & & & \\
\hline
\end{tabular}

Note: $f_{\mathrm{c}}$ and $f_{\mathrm{t}}$ are the compressive and tensile strength of concrete respectively; $f_{\mathrm{y}}$ and $f_{\mathrm{u}}$ are the yield and ultimate strength of steel respectively; $E_{\mathrm{c}}$ and $E_{\mathrm{s}}$ are the Young s modulus of concrete and steel.

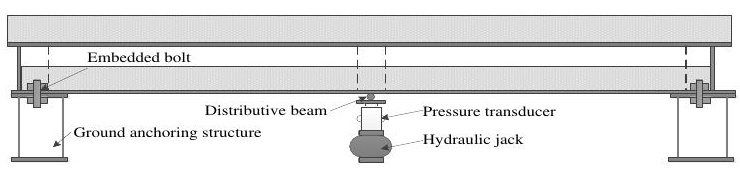

Fig. 4 Setup of test specimen

The vertical displacements was measured by displacement meters(LVDT) and the interlayer slip was measured by several dial indicators in the longitudinal direction. The locations of the LVDTs and the dial indicators were showed in Fig. 5.

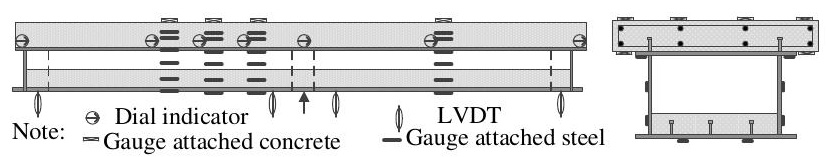

Fig. 5 Arrangement of measuring points

During testing, the cracking process, including position, direction and width, were also recorded in detail. The crack width was measured at several key load steps by an intelligent crack observation device (F61 Intelligent crack width tester).

In order to remove the gaps between specimens and loading machine, all specimens were preloaded before the formal test. After that, monotonic loading was applied by the hydraulic jack. The loading was controlled by force. The load increment imposed was $10 \mathrm{kN}$ for each stage at the initiation of the test; when the cracking load was approached, the load increment was reduced to 5 $\mathrm{kN}$ to observe the development of cracks. After concrete cracking, the load increment was improved to $20 \mathrm{kN}$, and when the load reached approximately $80 \%$ of the expected ultimate bearing capacity, the load increment for each stage was reduced to $10 \mathrm{kN}$ until the specimens failed. The loading was held constant for 2 min when reaching the default during each stage.

\section{Test results analysis}

There were three distinct stages in a typical composite beam failure process. Stage I was the stage before concrete cracking, and the materials behaved mainly elastically in this stage. Stage II corresponded to the stage in which concrete had been cracked but the steel profile had not yet yielded. In this stage, the bending stiffness of beams was weakened clearly, and non-linearity started to show in the load-deformation relationship curves. Generally, the ending of this stage is considered as the elastic limit. A composite beam in service is usually in stage II. Stage III started with the yielding of steel profile edge and ended with the failure of beams. In this stage, the bending stiffness of beams is weakened further.

At the end of stage I, cracks first appeared on the top surface of concrete slab at the mid-span position, and the initial tiny cracks did not change the bending stiffness of specimens obviously. In stage II, the steel beam was in the elasticity stage: the nonabsolutely linear behaviors and the stiffness declines were exhibited. The ultimate bearing capacity in this stage was 
approximately $0.8 P_{\mathrm{u}}$ (where $P_{\mathrm{u}}$ was the ultimate bearing capacity). In stage III, the deformation increased rapidly, and the bearing capacity began to decline at the end of this stage. Fig. 6 shows the failure states of CLB1, which exhibited "flexural failure"

\subsection{Load-deflection relation}

The load-deflection curves of three specimens are shown in Fig. 7. It reflects the relationship between load and deflection at the position of $200 \mathrm{~mm}$ distance from the mid-span.The duration time of stage I was very short in the action of negative bending moment for both CLB and DCB. When the load reached $35.7 \mathrm{kN}$, the initial crack appeared in DCB. The corresponding cracking loads of CLB1 and CLB2 were $70.71 \mathrm{kN}$ and $69.43 \mathrm{kN}$ respectively. The cracking loads of the two CLBs were approximately two times that of DCB.

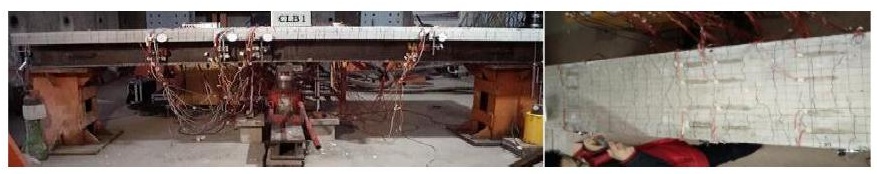

(a) Bending state

(b) Cracking state

Fig. 6 Failure states of the CLB

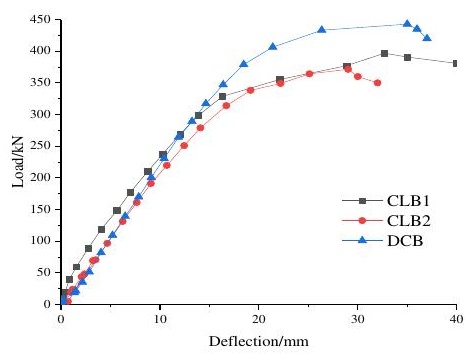

Fig. 7 Load-deflection relationship curves

From load-deflection curves, the non - linearity is obvious of both CLBs and DCB. The ultimate bearing capacity of DCB was greater than those of CLBs, but the elastic ultimate bearing capacity of CLBs were basically equivalent to DCB.

\subsection{Cracking}

In this and the following sections, take CLB1 and the DCB as examples for discussion because the distribution and development of cracks, strain, and stress of the CLB2 were similar to those of CLB1. Fig. 8 shows the crack development at critical loads in stages I and II. The development curves of the maximum crack width as the load increased were showed in Fig. 9. The shapes of the cracks reflect that the cracks were induced by a negative bending moment. Moreover, the symmetric distribution of crack around mid-span indicates that the loads were exerted symmetrically and equivalently around the mid-spans of the specimens.

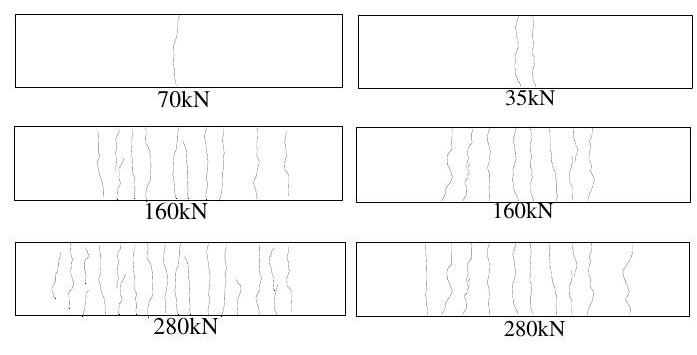

(a) CLB1

(b) DCB

Fig. 8 Crack formation and distribution

Due to different connector configurations, CLB1 exhibited flexural cracks, while DCB exhibited tensile cracks. More cracks were detected in CLB1 but the maximum crack width in CLB1 was always smaller than that of DCB.

It can be concluded that the crack resistance of the concrete slab could be greatly improved with the laminated action compared to the composite action The spacing between the main transverse cracks was almost $100 \mathrm{~mm}$ in CLB1 and $150 \mathrm{~mm}$ in $\mathrm{DCB}$, which was close to the spacing of stirrups in corresponding specimens.

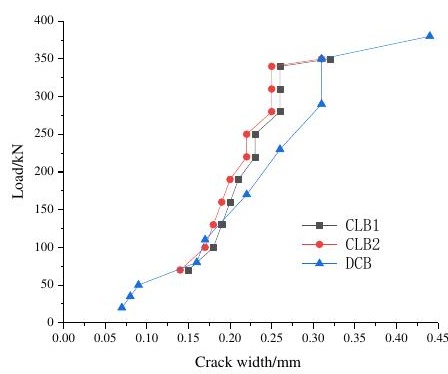

Fig. 9 Curves of load-maximum crack width

\subsection{Distribution of strain and plastic neutral axis}

The mechanical properties of composite beams were depend largely on the position of neutral axis in cross sections. The strain distribution along the cross-sectional height at the position of $200 \mathrm{~mm}$ away from the mid-span at different loading stages were showed in Fig. 10.

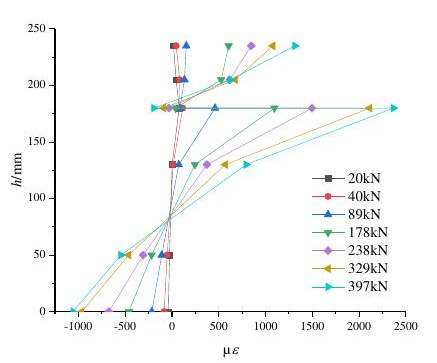

(a) Strain distributions in CLB1

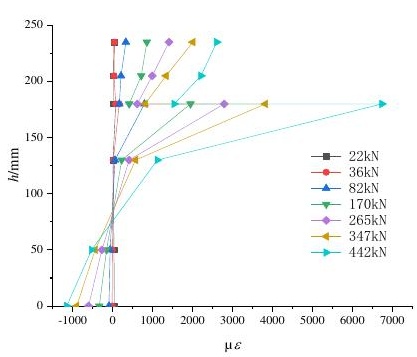

(b) Strain distributions in DCB
Fig. 10 Sectional strain distributions

In Fig. 10(a), the slip occurred under a low load level in CLB1, and before that, the strain distribution along the section was linear and satisfied the assumption of a plane section. The top and bottom beams still worked together as a whole after slip occurred excepting for the change of neutral axis. In the middle stage of loading, the bottom of the top beam began to be compressed, and at the same time, two neutral axes appeared in the cross section. It is because the initial bond between top and bottom beams was damaged as load increasing. After that, the top and bottom beams in CLB1 were bent and deformed respectively around their own neutral axes under the premise of keeping the same vertical deformation. The strain distribution along the cross-sectional height of DCB is shown in Fig. 10(b). The difference from the strain distribution of CLB1 was that there was only one neutral axis during the entire loading process, indicating that the top and bottom beams were always a whole structure in the process of stress.

\subsection{Relative slip}

Measured the maximum slip-load curves were presented in Fig. 11. As shown in Fig. 11, the maximum slippages of CLB1 and CLB2 were approximately two times greater than that of DCB. The slippage distribution along the length of specimens CLB1 and DCB is presented in Fig. 12(a) and (b) respectively, where the horizontal axis $l$ is the distance from the mid-span to the measured section. It indicates clearly from Fig. 12(a) and (b) that the slippage was distributed non-linearly along the longitudinal of specimens. This is because the slippage was extremely minor and affected by many factors such as stud weld quality, compaction rate of concrete, and eccentric loading, etc. The greatest slippages of CLB1 and DCB appeared at different positions. For CLB1, the greatest slippage was at the ends of specimen, but for $\mathrm{DCB}$, it was at the position of $1 / 4$ or $3 / 4$ of specimen's span.

\section{Analytical studies of the CLB}




\subsection{Sectional bending stiffness}

Bending stiffness of a cross-section refers to the ability of a member to resist changes in its curved shape. It is the basis for analyzing the deflection and deformation of a structure under load and the ductility of the structure, and is also used in calculating the crack width of a concrete structural member. Therefore, the bending stiffness is very important for the structural mechanical performance analysis.

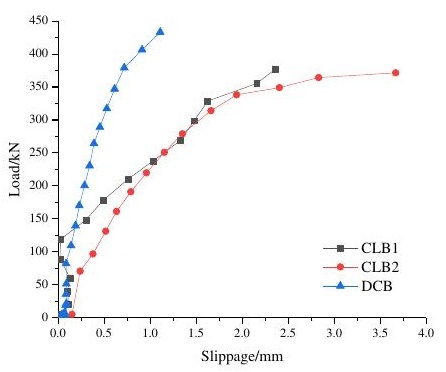

Fig. 11 Load-slippage relationship

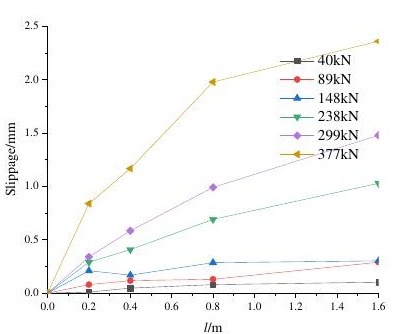

(a) CLB1

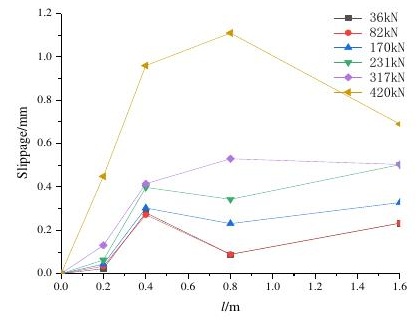

(b) DCB
Fig. 12 Load-slippage relationship

The cross-sectional bending stiffness calculation formulas of laminated beams without considering the friction effect have been given in the material mechanics [30]. However, before interface slip appears, the friction force in the rough interface will hinder the slippage and increase the bending stiffness of beams. Therefore, the calculation formulas of bending stiffness considering the action of friction will be obtained through the theoretical analysis in this section.

The micro-segment analysis model of CLB under the action of negative bending moment is shown in Fig. 13. $B_{1}, E_{1}, A_{1}$ and $I_{1}$ are the bending stiffness, elastic modulus, cross-sectional area and moment of inertia of the top beam, respectively, and the relevant parameters of the bottom beam are $B_{2}, E_{2}, A_{2}$ and $I_{2}$, respectively.

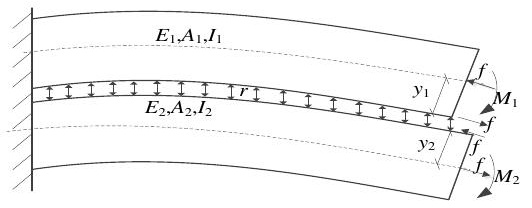

Fig. 13 Micro-segment analysis model

Under the action of the interface friction force $f$, the resistance bending moments $M_{\mathrm{f} 1}$ and $M_{\mathrm{f} 2}$ generated at the neutral axis positions of the top and bottom beams can be expressed as:

$M_{f i}=f y_{i}$

where $i=1,2$. The $y_{1}$ and $y_{2}$ are the distances from the neutral axis of top and bottom beams to the laminated interface.

The bending curvature $\phi_{f i}$ generated by the resistance bending moments to the top and bottom beams are: $\phi_{f i}=\frac{M_{f i}}{E_{i} I_{i}}$

In CLB, the bending stiffness of bottom beam is greater than that of top beam, which results in $\phi_{f 1}>\phi_{f 2}$. As shown in Fig. 14, the top and bottom beams will separate under the resistance bending moment action. Under the action of URSP connectors, CLB can prevent the separation of top and bottom beams effectively, and keep the curvature of top and bottom beams consistent. Therefore, the smaller one of $\phi_{f 1}$ and $\phi_{f 2}$ is taken as the influence of the resistance bending moment on the bending curvature of cross-section.

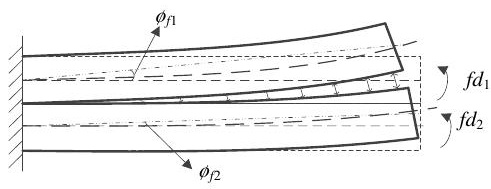

Fig. 14 Bending shape of CLB

The top and bottom beams' resistance strains $\varepsilon_{1}$ and $\varepsilon_{2}$ caused by the interfacial friction force $f$ are:

$\varepsilon_{i}=\frac{f}{E_{i} A_{i}}$

The bending curvature caused by the resistance strain on the cross-section is $\phi_{f}^{\prime}$, expressed as:

$\phi_{f}^{\prime}=\frac{\varepsilon_{1}+\varepsilon_{2}}{y_{1}+y_{2}}$

Combining equations (3) and (4), the resistance curvature of cross section generated by friction resistance is $\phi$ :

$\phi=\frac{f}{y_{s c}}\left(\frac{1}{E_{1} A_{1}}+\frac{1}{E_{2} A_{2}}\right)+\frac{f y_{2}}{E_{2} I_{2}}$

where the $y_{\mathrm{sc}}=y_{1}+y_{2}$.

The additional bending stiffness $K_{f}$ caused by the friction can be expressed as:

$K_{f}=\frac{f}{y_{s c}}\left(\frac{1}{E_{1} A_{1}}+\frac{1}{E_{2} A_{2}}\right)+\frac{f y_{2}}{E_{2} I_{2}}$

The sectional bending stiffness in the negative bending moment region, $B$ can be expressed as:

$B=B_{1}+B_{2}+K_{f}$

\subsection{Calculation of ultimate flexural capacity}

As shown in Fig. 15, there are two plastic neutral axes in CLB, the bending moment of CLB is the bending moment sum of top and bottom beam.

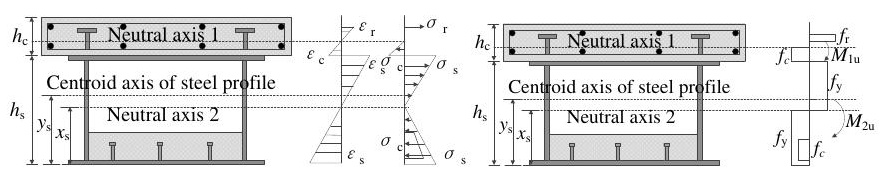

(a) Calculation model of $M_{\mathrm{e}}$

(b) Calculation model of $M_{\mathrm{u}}$

Fig. 15 Calculation model of ultimate bending moment

A calculation model for ultimate flexural capacity was proposed based on the transformed section method and the simplified plastic theory. It is reasonable to assume that: (1) ignoring the tensile strength of concrete; (2) the 
cross section as a compact one, meaning that the steel profile would not lose stability under pressure before reaching the cross-sectional carrying capacity ; and (3) without considering the natural adhesion force of laminated interface.

The whole process of stress can be divided into three distinct stages [31], the stress and strain distribution along cross-section height at each stage are shown in Fig. 15.

(1) The stage before concrete $\operatorname{cracking}\left(M \leq M_{\mathrm{cr}}\right)$

The top reinforced concrete slab of CLB will crack under a small negative bending moment, and the cracking moment can be expressed as [32]:

$$
M_{\mathrm{cr}}=\frac{\gamma_{m} W_{0} f_{t}}{n}
$$

where the $\gamma_{\mathrm{m}}$ is the resistance coefficient of section, it is 5.5 for a rectangular concrete slab; $W_{0}$ is the cross-section resistance, $W_{0}=2 I_{1} / h_{\mathrm{c}}, h_{\mathrm{c}}$ is the height of top beam; $f_{\mathrm{t}}$ is the tensile strength of concrete; $n$ is the coefficient of stiffness distribution, $n=B_{1} /\left(B_{1}+B_{2}\right)$.

(2) The stage of elasticity working $\left(M_{\mathrm{cr}} \leq M \leq M_{\mathrm{y}}\right)$

After concrete cracking, the steel beam is still in the elastic working stage. In this stage, CLB is approximately in an elastic working stage. The neutral axis moves down with the increase of moment, the deflection and rotation increase fast.

$M_{y}=M_{1 y}+M_{2 y}$

where $M_{1 y}$ and $M_{2 y}$ are the elasticity ultimate bending moments of top and bottom beams, respectively.

$M_{1 y}=A_{r} f_{r}\left(\frac{h_{c}}{2}-a-\frac{d}{2}\right)+f_{c} b_{c} \frac{h_{c}^{2}}{8}$

$M_{2 y}=\frac{f_{y} I_{2}}{h_{s}-x}$

where $A_{\mathrm{r}}$ is the area of reinforcing steel bar, $b_{\mathrm{c}}, h_{\mathrm{c}}$ is the width and height of top beam respectively, $h_{\mathrm{s}}$ is the height of steel beam, $d$ is the diameter of reinforcing steel bar, and $a$ is the thickness of protective layer, $f_{\mathrm{r}}$ is the tensile strength of reinforcing steel bar.

(3) The stage of plasticity working $\left(M_{\mathrm{y}} \leq M \leq M_{\mathrm{u}}\right)$

When the tensile steel profile reaches yield strength $f_{\mathrm{y}}$, the bending moment $M_{\mathrm{y}} \approx(0.8 \sim 0.9) M_{\mathrm{u}}$. The yield range of steel beam expands further with the increase of moment. Based on the theory of total plasticity, the ultimate moment $M_{1 \mathrm{u}}$ of top beam and $M_{2 \mathrm{u}}$ of bottom beam can be expressed as:

$M_{1 \mathrm{u}}=a_{1} f_{c} b_{c} \frac{x_{c}{ }^{2}}{2}+f_{r} \frac{A_{r}}{2}\left(x_{c}-a\right)+f_{r} \frac{A_{r}}{2}\left(h_{c}-x_{c}-a\right)$

$M_{2 u}=W_{x} f_{y}+a_{1} f_{c} b_{c 2} h_{c 2}\left(x_{s}+\frac{y_{w}}{2}-t_{f 2}-\frac{h_{c 2}}{2}\right)$

where $x_{\mathrm{c}}$ is the thickness of the compressed concrete in the top beam, $W_{\mathrm{x}}$ is the cross-sectional bending coefficient of steel profile, $b_{\mathrm{c} 2}$ and $h_{\mathrm{c} 2}$ are the width and height of bottom concrete slab respectively, $x_{\mathrm{s}}$ and $y_{\mathrm{w}}$ are shown in Fig. 15(b)

\subsection{Slippage distribution analysis}

The bending stiffness is effected by the friction force, and the friction action will also affect the structural slippage. In this section, the theoretical analysis on the interface slip of simply supported CLB under the uniform and the reverse concentrated load is carried out to obtain the slip distribution equations along the beam's longitudinal.

Considering the symmetry of structure and the slippage distribution, analysis is conducted by taking the mid-span as the coordinate origin, and the range of $x>0$ as the analysis object (Fig. 16).

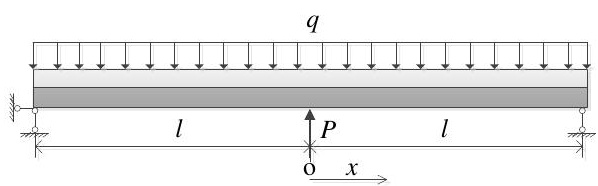

\subsubsection{Slip under uniform load}

The micro-segment deformation model for negative bending moment of CLB under uniform load is shown in Fig. 17. In Fig. 17, $T_{1}$ and $T_{2}$ are the tensile forces of top and bottom beams, respectively; $V_{1}$ and $V_{2}$ are the shear forces of top and bottom beams, respectively; and $r$ is the pressing force on laminated interface.

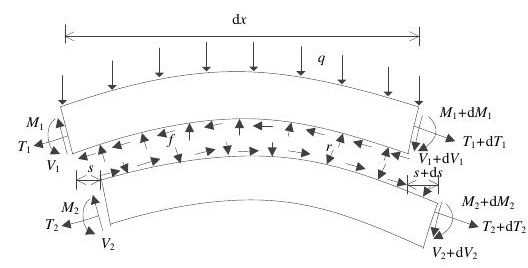

Fig. 17 Micro-segment deformation model under uniform load

The friction $f$ existing on the laminated interface can be expressed as:

$f=\mu r$

where $\mu$ is the coefficient of friction.

The distribution of load is determined according to the bending stiffness of top and bottom beams [33-34], and the pressing force between the laminated interfaces is:

$r=\frac{q E_{2} I_{2}}{E_{1} I_{1}+E_{2} I_{2}}$

The bending moments on the left side of top and bottom beam units are taken and sorted out respectively:

$-\frac{d M_{1}}{d x}+\left(V_{1}+d V_{1}\right)+(q-r) \frac{d x}{2}+\mu r y_{1}=0$

$-\frac{d M_{2}}{d x}+\left(V_{2}+d V_{2}\right)+r \frac{d x}{2}+\mu r y_{2}=0$

Assuming that the distance of the micro-segment to the right support to be $a(a=l-x)$ :

$V_{1}+d V_{1}+V_{2}+d V_{2}=-q x$

According to the assumption (1):

$\phi=\frac{M_{1}}{E_{1} I_{1}}=\frac{M_{2}}{E_{2} I_{2}}$

where $\phi$ is the curvature of cross-section, as well as the top and bottom beams.

The compressive strain at the bottom of the top beam and the tensile strain at the top of the bottom beam are:

$\varepsilon_{c}=\phi y_{1}-\frac{T_{1}}{E_{1} A_{1}}$

$\varepsilon_{t}=\phi y_{2}+\frac{T_{2}}{E_{2} A_{2}}$

Combining the above formulas, there is:

$\frac{d \phi}{d x}=\frac{\mu r y_{s c}-q x}{E I}$

Fig. 16 Schematic diagram of structural analysis 
where $E I=E_{1} I_{1}+E_{2} I_{2}$.

The expression of the first derivative of simply supported CLB slip strain is:

$\varepsilon_{s}^{\prime}=\frac{\mu r y_{s c}-q x}{E I} y_{s c}+\mu r\left(\frac{1}{E_{1} A_{1}}+\frac{1}{E_{2} A_{2}}\right)$

The boundary conditions of slip and slip strain of simply supported CLB are:

$$
\left\{\begin{array}{l}
\left.\varepsilon_{s}\right|_{x=l}=0 \\
\left.s\right|_{x=0}=0
\end{array}\right.
$$

The slip distribution equation of laminated interface under uniform load can be expressed as:

$s=\frac{\frac{\mu r y_{s c} x(x-2 l)}{2}-\frac{q x\left(x^{2}-3 l^{2}\right)}{6}}{E I} y_{s c}+\mu r x(x-2 l)\left(\frac{1}{2 E_{1} A_{1}}+\frac{1}{2 E_{2} A_{2}}\right)$

\subsubsection{Slip under concentrated load}

Similar to the derivation under the uniform load, the slip distribution equation of laminated interface under concentrated load can be obtained:

$s=\frac{P}{2 E I} y_{s c}\left(\frac{x^{2}}{2}-x l\right)$

where $P$ is the concentrated load.

\section{Comparisons}

\subsection{Slip comparison}

In the experiment, the friction coefficient $\mu$ between the laminated interface was not measured. According to the relevant research [35], $\mu=0.7$ was adopted to calculate the slip distribution. Theoretical predictions and experimental responses of CLB1 were shown in Fig. 18, the " $T$ " represents theoretical predictions and the " $M$ " represents measured experimental responses.

From Fig. 18, it can be seen that when the load is $60 \mathrm{kN}$, the measured results are only about half of the analysis results, mainly due to the initial bonding between the top and bottom beam is still strong during the initial loading. As the load increasing, the measured values are about $10 \%$ smaller than the analytical values, which may still result from the interfacial adhesion or frictional force. By comparing the slip test results with the analysis results, it can be concluded that the slip distribution equations (23) and (24) considering the interfacial friction force is effective for analyzing the slip distribution of CLB.

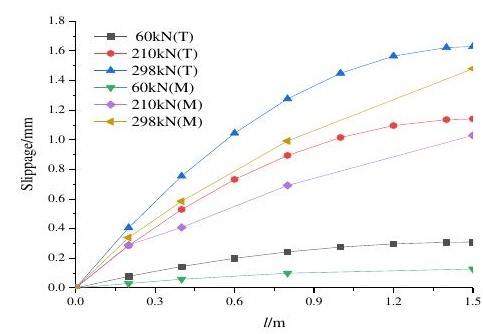

Fig. 18 Comparison of slip distribution curves

\subsection{Ultimate flexural capacity comparison}

The critical bending moments obtained from tests and predicted by theoretical analysis are tabulated in Table 2. It can be found that the Eqs. (6)-(11) and the calculation formulas in reference [7] can predict the critical bending moments of CLB and DCB well.

\section{Conclusions}

Experimental and analytical investigation on the mechanical behaviors of the laminated action steel-concrete composite beam has been conducted in this paper. The mechanical properties including development of cracks, slippage, ultimate bearing capacity of CLB and DCB were analyzed and compared. On the basis of theoretical analysis, the calculate formulas for bending stiffness of cross-section were given. Under negative bending moment, the slip distribution equations considering frictional force of simply supported CLB were conducted. The calculate formulas for ultimate bending carrying capacity were obtained. And the theoretical predictions and experimental responses were good agreement. The following conclusions may be drawn from the present study:

- The static failure mode of the CLB in the negative bending moment region is flexural failure, similar to that of the DCB. In the inelastic stage after

Table 1

Experimental and Analytical Results of Critical Bending Moments of Specimens

\begin{tabular}{|c|c|c|c|c|c|c|c|c|}
\hline \multirow{2}{*}{ Limit state } & \multicolumn{4}{|c|}{ CLB } & \multicolumn{4}{|c|}{ DCB } \\
\hline & Eqs. & $(\mathrm{kN} \cdot \mathrm{m})$ & Measured $(\mathrm{kN} \cdot \mathrm{m})$ & Eqs. to measured & Eqs. & $(\mathrm{kN} \cdot \mathrm{m})$ & Measured $(\mathrm{kN} \cdot \mathrm{m})$ & Eqs. to measured \\
\hline \multirow{2}{*}{ Cracking bending moment } & \multirow{2}{*}{\multicolumn{2}{|c|}{54.7}} & 53.0 & 1.03 & \multirow{2}{*}{\multicolumn{2}{|c|}{30.2}} & \multirow[t]{2}{*}{26.8} & \multirow[t]{2}{*}{1.13} \\
\hline & & & 52.1 & 1.05 & & & & \\
\hline \multirow[t]{2}{*}{ Elastic limit bending moment } & \multirow{2}{*}{\multicolumn{2}{|c|}{239.5}} & 246.5 & 0.97 & \multirow{2}{*}{\multicolumn{2}{|c|}{269.7}} & \multirow[t]{2}{*}{260.4} & \multirow[t]{2}{*}{1.04} \\
\hline & & & 235.5 & 1.02 & & & & \\
\hline \multirow[t]{2}{*}{ Plastic limit bending moment } & \multirow{2}{*}{\multicolumn{2}{|c|}{291.9}} & 297.6 & 0.98 & \multirow{2}{*}{\multicolumn{2}{|c|}{318.6}} & \multirow[t]{2}{*}{332.0} & \multirow[t]{2}{*}{0.96} \\
\hline & & & 278.5 & 1.04 & & & & \\
\hline
\end{tabular}

concrete cracking, the ultimate bending capacity of CLB is almost equivalent to that of DCB.

- Concerning the cracks development of top concrete slab, it can be deduced that CLB exhibit flexural cracks, while DCB exhibit tensile cracks. Comparing with DCB, the cracking moment of CLB is nearly doubled, and the maximum crack width is reduced by about $50 \%$. .

- The slip distribution formulas which are developed by considering the interfacial friction force are effective for analyzing the slip distribution of CLB. And the formulas obtained by theoretical analysis can predict the flexural bearing capacity of CLB well.

\section{Acknowledgments}

The work is supported by key project of Natural Science Foundation of Hebei Education Department, China (Contact No. ZD2018025) and the Shijiazhuang Tiedao University's Graduate Innovation Fund Project, China(No.YC 2018001)

\section{References}

[1] Fan, J.S., Gou, S.K., Ding, R., et al., "Experimental and analytical research on the flexura behaviour of steel ECC composite beams under negative bending moments", Engineering Structures, 210, 2019.

[2] Wang, Y., Li, T., "Numerical analysis of forces in negative bending region of steel-concrete composite beams". Sensors \&amp; Transducers, 168(4), 2014.

[3] Śledziewski Krzysztof,Górecki Marcin. "Finite element analysis of the stability of a sinusoidal web in steel and composite steel-concrete girders", Materials (Basel, Switzerland), 13(5), 2020.

[4] Reiner, S., "Bridges with double composite action", Structural Engineering International, 6(1), 32-36, 1996

[5] Steven L. Stroh, Rajan Sen, "Steel bridges with double-composite action", Transportation Research Record, 1696, 299-309, 2000.

[6] Xu, C., Su, Q.T., Wu, C., Sugiura, K., "Experimental study on double composite action in the negative moment region of two-span continuous composite box girder", J. Constr. Steel Res, 67(10), 1636-1648, 2011

[7] Duan, S.J., Huo, J.H., Zhou, Q.D., "Research on calculation method of ultimate bearing capacity of double steel-concrete composite beams", Journal of Shijiazhuang Railway 
Institute (Natural Science Edition), (04), 1-5, 2007

[8] Duan, S.J., Niu, R.M., Wang, W.C., et al., "Study on crack expansion mechanism and crack width of the double composite continuous beam", Journal of the China Railway Society, 34(12), 96-101, 2012

[9] Duan, S.J., Zhou, Q.D., Wang, H.L., et al., "Experimental study on bearing capacity of the double composite continuous beam", Journal of Railway Science and Engineerin, (05), 12-17, 2008 .

[10] Duan, S.J., Shao, X.H., Niu, R.M., et al., "Experimental study on interface slip of the steel-concrete double composite continuous beam", Highway Traffic Science and Technolog, 26(07), 95-99, 2009

[11] Ryu Hyung-Keun, Kim Young-Jin, Chang Sung-Pil., "Crack control of a continuous composite two-girder bridge with prefabricated slabs under static and fatigue loads", Eng Struct, 29(6), 851-864, 2007

[12] Chang-Su Shim, Hyung-Keun Ryu, Chul-Hun Chung, et al., "Inelastic behavior of a continuous composite box girder bridge with prefabricated slabs", Composite Construction in Steel and Concrete V, Proceedings of the 5th International Conference, (02), 57-66, ASCE, July, 2006.

[13] He, J., Liu, Y.Q., Chen, A.R., et al., "Experimental study on inelastic mechanical behaviour of composite girders under hogging moment", J. Constr. Steel Res., 66(1), 37-52, 2010.

[14] Dong, Y.Y., Xu, Y., "Research on anti-cracking design method of steel-concrete composite beams in negative moment zone", Proceedings of 2019 International Conference on Virtual Reality and Intelligent Systems (ICVRIS 2019), Volume II, 143-146, 2019.

[15] L. K. Varshney, K. A. Patel, Sandeep Chaudhary, et al., "An efficient and novel strategy for control of cracking, creep and shrinkage effects in steel-concrete composite beams", Structural Engineering and Mechanics, 70(6), 751-763, 2019.

[16] Nie, J.G., "Application of steel-concrete composite structure in ocean engineering", Steel Construction, 35(1), 20-33, 2020.

[17] Ding, Y., Dai, X.M., Yan. J.B., "Development and behaviors of slip-released novel connectors in steel-concrete composite structures", Advanced Steel Construction, 15(1), 30-36, 2019.

[18] Zhang, Y.L., "Experimental and theoretical study on the mechanical properties and cracking control of steel-concrete composite beams in negative bending moment region", Ph.D. Dissertation, Beijing Jiaotong University, 2009.

[19] Wang, Y.H., Yu, J., Liu, J.P., et al., "Experimental study on assembled monolithic steel-prestressed concrete composite beam in negative moment", Journal of Constructional Steel Research, 167, 2020

[20] Chen, S.M., Wang, X.D., Jia, Y.L., "A comparative study of continuous steel-concrete composite beams prestressed with external tendons: experimental investigation", J. Constr. Steel Res., 65(7), 1480-1489, 2009.

[21] Eray Baran,Cem Topkaya., "Behavior of steel-concrete partially composite beams with channel type shear connectors", Journal of Constructional Steel Research, 97, 2014.

[22] Nie, J.G., Wei, J., "The actual performance of shear connector in steel-concrete composite beam", Journal of Zhengzhou Institute of Technology, 04, 43-47, 1991.

[23] Liu, W.H., Chang, D.B., "Experiment study on the crack resistance of composite beams", Journal of Jilin Architectural and Civil Engineering, 03, 1-3, 2008.

[24] Nie, J.G., Tao, M.X., Nie, X., et al., "New technique and application of uplift- restricted and slip-permitted connection", China Civil Engineering Journal, (04), 7-14, 58, 2015.

[25] Nie, J.G., Li, Y.X., Tao, M.X., et al., "Resistance test of new type- uplift-restricted and slip-permitted connector", China Journal of Highway and Transport, 27(04), 38-45, 2014

[26] Nie, J.G., Ma, Y., "Experimental study on the resistance performance of uplift-restricted and slip-permitted studs connectors", Special Structural Periodicals, 32(03), 6-12, 2015.

[27] Duan, S.J., Niu, R.M., An, R.M., et al., "Steel-concrete composite-laminated beam", ZL 20162 1090805. 0, 2017.04.19. Chinese Patent.

[28] Nie, J.G., Tao, M.X., Wu, L.L., et al., "New progress in the study of steel-concrete composite bridge", China Civil Engineering Journal, 45(06), 110-122, 2012.

[29] Nie J G, Wang J J, Guo S K, et al. "Technological development and engineering application of novel steel-concrete composite structure", Frontiers of Structural and Civil Engineering. 2019, Vol.13 (1), pp.1-14

[30] Sun, X.F., Fang, X.S., Guan, L.T., "Material mechanics 1", Beijing: Higher Education Press, 2009.

[31] Guo, Z.H., "Principles of reinforced concrete(3nd)", Beijing: Tsinghua University Press, 2013

[32] GB50010(2010), Code for Design of Concrete Structures, China Architecture \& Building Press, Beijing, China, 2010

[33] Shu, X.P., "Study on contact pressure of beams considering friction[J]. Mechanics and Practice", 13(05), 23-26, 1991.

[34] Luo, K.B., "Analysis of contact pressure between laminated beams considering shear deformation", Mechanics and Practice, 9(02), 34-38, 1987.

[35] Su, Q.T., Du, X., L, C.X. and Jiang, X., "Basic physical parameter test of the steel and concrete interface", Journal of Tongji University(Natural science), 44(04), 499-506, 2016. 


\title{
SEISMIC BEHAVIOR OF BIAXIAL HOLLOW REINFORCED CONCRETE SLAB TO CONCRETE-FILLED STEEL TUBULAR COLUMN CONNECTIONS
}

\author{
Chao Gong ${ }^{1}$, Zhao-Xin Hou ${ }^{1}$, Guo-Zhong Cheng ${ }^{2,}$,, Y. Frank Chen ${ }^{2}$ and Wei-Qiao Liang ${ }^{1}$ \\ ${ }^{1}$ Central Research Institute of Building and Construction Co., Ltd, Beijing 100088, China \\ ${ }^{2}$ Key Laboratory of New Technology for Construction of Cities in Mountain Area (Chongqing University), Ministry of Education, Chongqing 400045, China \\ *(Corresponding author: E-mail: chengguozhong@cqu.edu.cn)
}

\section{A B S T RA C T}

This paper discusses the seismic behavior of biaxial hollow reinforced concrete (RC) slab to concrete-filled steel tubular (CFST) column connections. Seven scaled specimens were tested under cyclic loading, where the axial compressive load applied on CFST column was selected as the parameter. Both exterior and interior joints were investigated. Two types of failure modes, i.e., beam failure and column failure, were observed. For specimens with beam failure, the failures are mainly caused by the fracture of top steel beam flange. For specimens with column failure, the failures are primarily triggered by the buckling of tube wall. The proposed composite slim floor system inherently meets the principle of strong joint-weak member. The ultimate story drift of all specimens is 2.2-2.8 times of plastic drift ratio specified in codes, and the equivalent damping coefficient at peak loads ranges from 0.12 to 0.19 , indicating the proposed composite slim floor system has a good seismic performance.
A R T I CLE H IST TORY

$\begin{array}{ll}\text { Received: } & 7 \text { November } 2019 \\ \text { Revised: } & \text { 18 May } 2020 \\ \text { Accepted: } & \text { 30 May } 2020\end{array}$

\section{K E Y W O R D S}

Hollow core slab;

Concrete filled steel tube (CFST);

Connection;

Seismic behavior;

\section{Introduction}

The earliest form of composite slim floor construction was used in the 1790s. Since the introduction in Scandinavia, the composite slim floor system (Fig. 1) has been widely used in Europe and the United States [1, 2]. As shown, a singly symmetrical I-beam with the wider bottom flange supporting a deep deck is used. The key feature is that the steel beam is encased in the slab, resulting in a flat appearance and a decreased beam depth. The steel beam is usually connected to the column through a shear plate and bolts (Fig. 1c), as indicated by the existed literature [3, 4]. The reinforced concrete (RC) slab prevents the steel beam from local buckling and eliminates the need of fire protection $[5,6]$. Therefore, the composite slim floor system is an innovative and alternative floor system to traditional RC floor system and traditional steel-concrete composite floor system. However, the thickness of composite slim floor is larger than that of traditional floor system, which increases the weight of floor system.

Extensive research has been conducted on this type of floor systems, particularly the integrated composite beam and the connection between composite slim floor and column. Nine simply-supported composite slim beams have been tested at Helsinki University of Technology, followed by the parametric studies on the flexural behavior of the beams [7], which indicated that interface slippage in composite slim beams with reinforced bars was much less and could be neglected. Additionally, a study on the behavior of the beam-to-column connections was performed at the same place [8], where a semi-comtinuous beam-column connection was investigated and classified as partial-strength. Experiments on eight specimens were carried out at Trento University to investigate the influences of reinforcement ratios and load conditions on the behavior of composite slim beams [9], which show that the reinforcement ratio has a significant influence on the loading capacity of the composite slim beams. The shear transfer mechanism provided by the shear connectors between steel beam and concrete slab has been also studied, which indicates that interface slippage in composite slim beams is negligible [10,11]. Besides the structural behavior, research has also been done on the fire resistance of composite slim floor systems and it shows that such systems have a good fire resistance $[10,11]$.

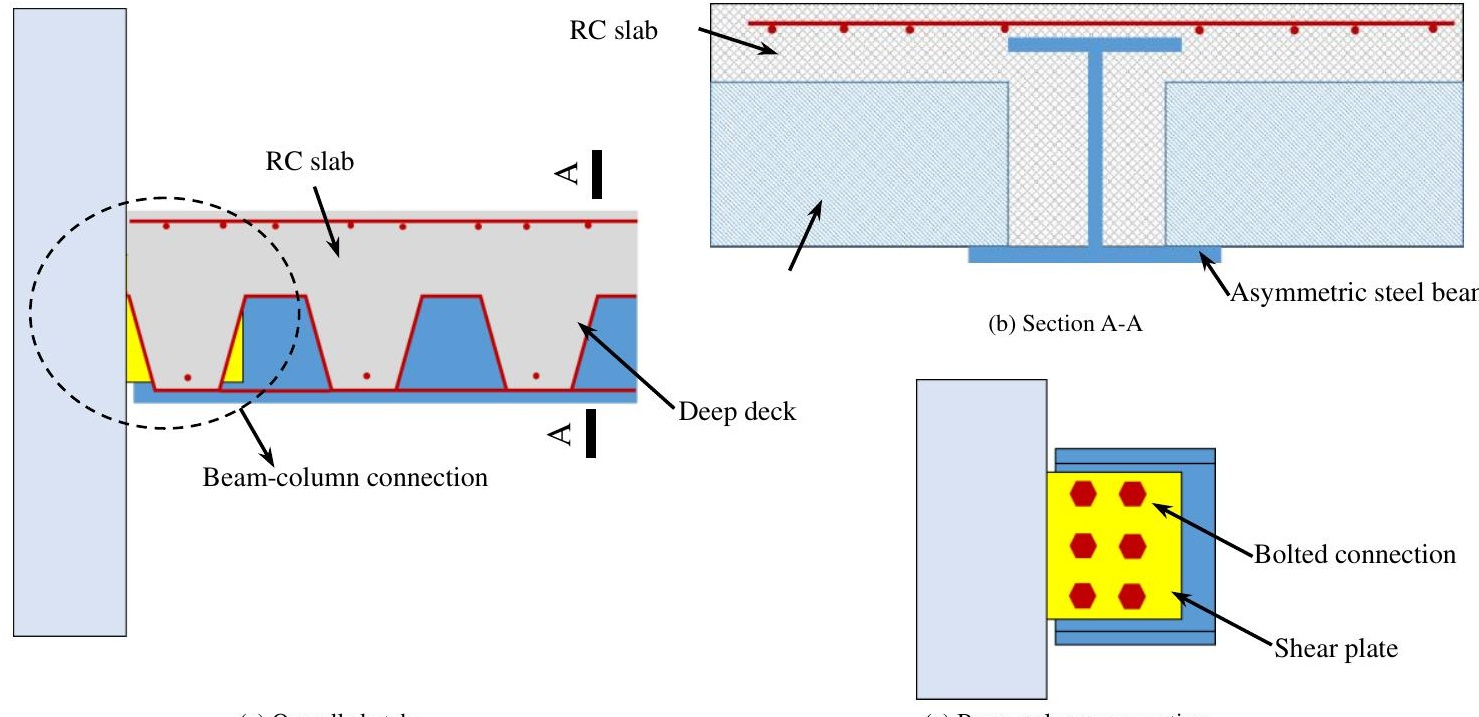

(a) Overall sketch

(c) Beam-column connection

Fig. 1 The composite slim floor system 


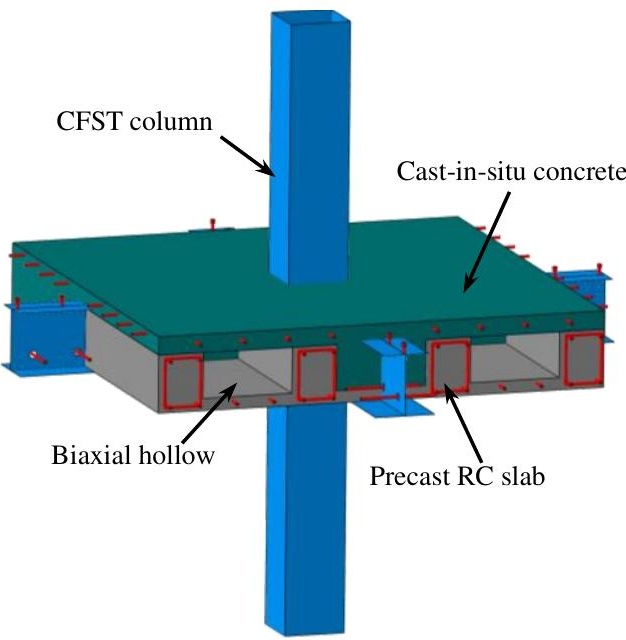

(a) Sketch

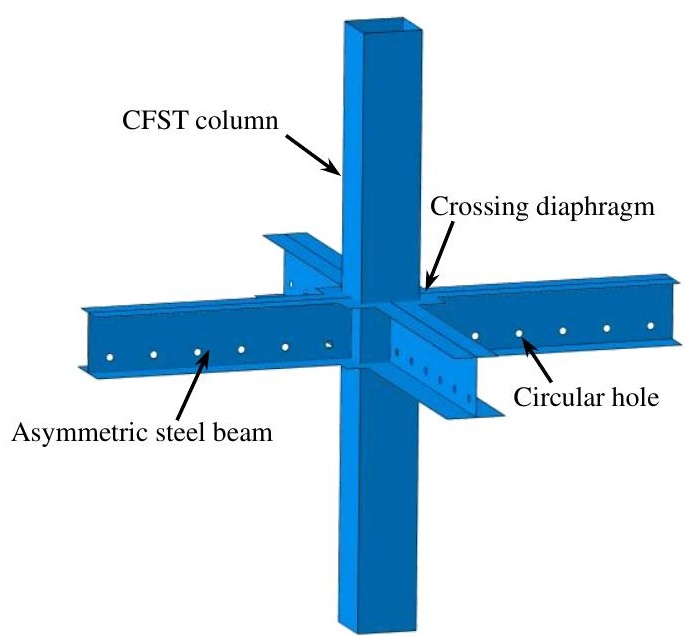

(b) Beam-column connection

Fig. 2 Proposed composite slim floor system

Deep decks or precast concrete (PC) slabs have been commonly used in the conventional composite slim floor system (Fig. 1). However, the design of PC slabs is usually governed by the width of concrete cracks and may be uneconomical. The deep decks, the depth of which is higher than $200 \mathrm{~mm}$, are not available in every place including China. Besides, the deep decks need to be protected from corrosion, which would increase the cost. Moreover, the traditional composite slim floor system is not suitable for moment resistance because of the semi-comtinuous beam-column connections. Therefore, the conventional composite slim floor system is not widely adopted in China. For these reasons, an improved composite slim floor system (Fig. 2) with the rigid beam-column connection is proposed. In this system, an asymmetric steel beam contained in the RC slab is used to minimize the floor depth similarly to the traditional composite slim floor system. The precast RC slabs are employed as the structural components as well as the permanent formwork. Biaxial hollow boxes are introduced to reduce the RC slab weight. The asymmetric steel beam is connected to the concrete filled steel tubular (CFST) column using the crossing diaphragms. As first attempt to study the seismic performance of the proposed floor system, this paper focuses on the seismic behavior of beam-column connection. Seven connection specimens were tested under cyclic loading. The influences of different testing parameters on the seismic behavior of connections were evaluated in detail.

\section{Experimental program}

\subsection{General information of specimens}

Seven connection specimens were tested under cyclic loading, where the details are shown in Fig. 3. As indicated, CFST columns are $200 \mathrm{~mm} \times 200$ $\mathrm{mm}$ in cross-section, $3200 \mathrm{~mm}$ long, and with $6 \mathrm{~mm}$ thick steel tube. The composite slim beam has the cross-section of $800 \mathrm{~mm}$ (width) $\times 270 \mathrm{~mm}$ (height) and the length of $2380 \mathrm{~mm}$ (Fig. 4). It consists of a singly-symmetrical steel I-beam, precast concrete, filler, cast-in-place (CIP) concrete, and rebars. The steel beam has a $36 \mathrm{~mm}$-diameter hole, with both perfobond shear connectors and shear studs adopted to transfer the shear force between RC slab and steel beam. The $14 \mathrm{~mm}$-diameter overlapped rebars pass through the circular hole and the shear studs having the diameter of $10 \mathrm{~mm}$ and the length of $40 \mathrm{~mm}$ were welded to the beam flange. Both the overlapped rebars and the shear studs were spaced evenly at $200 \mathrm{~mm}$. The beam flanges were made continuous with the crossing diaphragms, thus eliminating the uncertain effect caused by flange welds (Fig. 3(c)). The crossing diaphragms was designed according to Chinese code CECS 159: 2004 [12]. The average compressive strength of concrete is $35.4 \mathrm{MPa}$ for the RC slab and $38.6 \mathrm{MPa}$ for the CFST column, which were measured from six concrete cubes $(150 \mathrm{~mm}$ $\times 150 \mathrm{~mm} \times 150 \mathrm{~mm})$. To assess the effect of connection location, both exterior and interior joints (Fig. 5) were considered. The main parameter investigated is the axial compressive load applied on the CFST column. Additionally, the steel beam to CFST column joint was tested for comparison. Detailed steel material properties are listed in Table 1, and specimen details are listed in Table 2 where $n$ is the axial load ratio of CFST column defined as

$n=N /\left(f_{\mathrm{cc}} A_{\mathrm{c}}+f_{\mathrm{y}} A_{\mathrm{s}}\right)$

in which $N, f_{\mathrm{c}}, f_{\mathrm{y}}, A_{\mathrm{c}}$, and $A_{\mathrm{s}}$ are the axial compressive load applied on CFST column, the axial compressive strength of infilled concrete, the yield strength of steel tube, the cross-sectional area of infilled concrete, and the cross-sectional area of steel tube. 


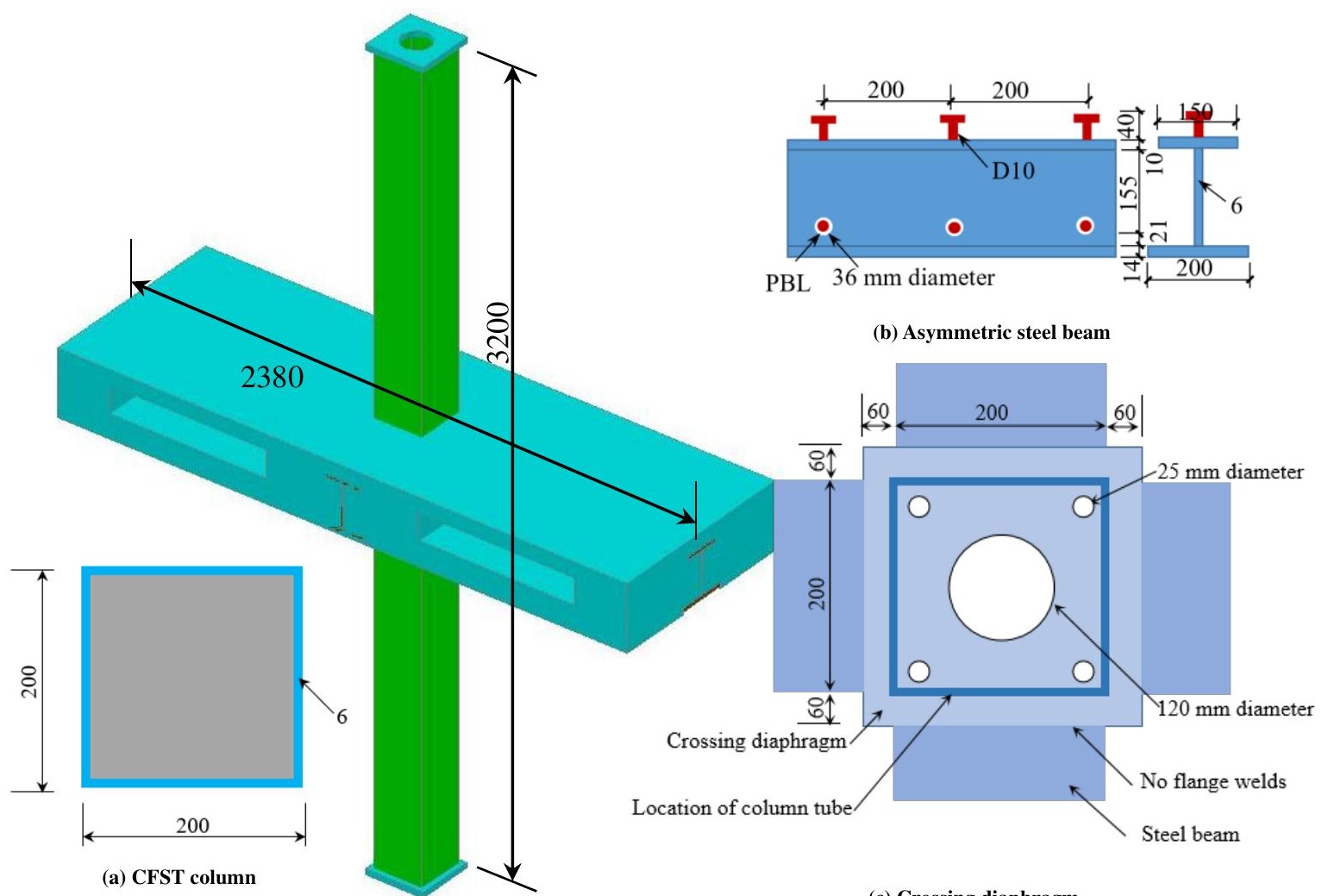

(c) Crossing diaphragm

Fig. 3 Details of specimen (unit: $\mathrm{mm}$ )

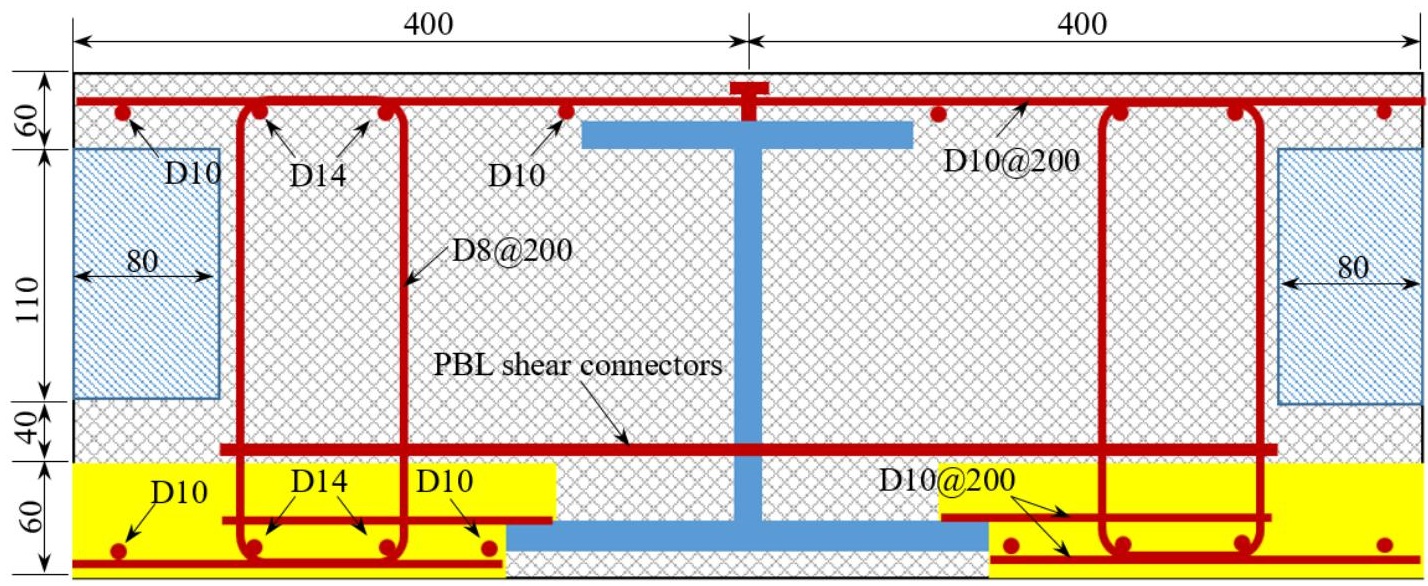

Fig. 4 Cross-section of RC slab

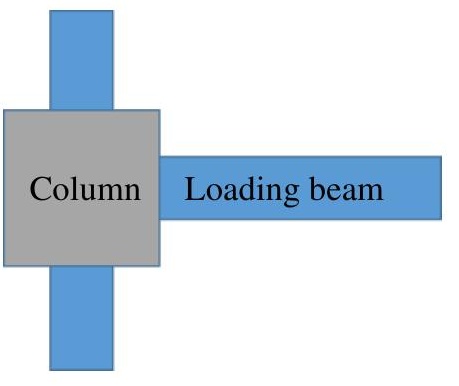

(b) Exterior joints

$$
\underset{\text { Loading direction }}{\longrightarrow}+
$$$$
\text { Loading beam Column Loading beam }
$$ 
Table 1

Material properties of steel

\begin{tabular}{ccc}
\hline Material label & Yield strength $f_{\mathrm{y}}(\mathrm{MPa})$ & Ultimate strength $f_{\mathrm{u}}(\mathrm{MPa})$ \\
\hline $10 \mathrm{~mm}$ deformed steel bar & 433 & 630 \\
$14 \mathrm{~mm}$ deformed steel bar & 436 & 620 \\
$6 \mathrm{~mm}$ thick steel plate & 382 & 508 \\
$8 \mathrm{~mm}$ thick steel plate & 356 & 388 \\
$10 \mathrm{~mm}$ thick steel plate & 367 & 513 \\
$14 \mathrm{~mm}$ thick steel plate & 510 \\
\hline
\end{tabular}

Table 2

Details of specimens (unit: $\mathrm{mm}$ )

\begin{tabular}{|c|c|c|c|c|c|}
\hline Specimen label & CFST column & Asymmetric steel beam & Connection location & $n$ & RC slab \\
\hline Specimen-1 & \multirow{7}{*}{$\begin{array}{l}\text { Width: } 200 \\
\text { Height: } 200 \\
\text { Thickness: } 6\end{array}$} & \multirow{7}{*}{$\begin{array}{l}\text { Height: } 200 \\
\text { Width: } \\
\text { 1. Top flange: } 150 \\
\text { Bottom flange: } 200 \\
\text { Thickness: } \\
\text { 1. Top flange: } 10 \\
\text { 2. Bottom flange: } 14 \\
\text { 3. Web: } 6\end{array}$} & Exterior joints & 0.5 & Without \\
\hline Specimen-2 & & & Exterior joints & 0.2 & With \\
\hline Specimen-3 & & & Exterior joints & 0.5 & With \\
\hline Specimen-4 & & & Exterior joints & 0.6 & With \\
\hline Specimen-5 & & & Interior joints & 0.2 & With \\
\hline Specimen-6 & & & Interior joints & 0.35 & With \\
\hline Specimen-7 & & & Interior joints & 0.5 & With \\
\hline
\end{tabular}

\subsection{Test set-up and instrumentation layout}

All columns and beams were pin-connected at their ends. The inflection points are likely to occur at the column mid-height and beam mid-span during a seismic event, which may be represented by pins. Details of the test set-up and instrumentation layout are shown in Fig. 6. The specimen was loaded by two $1000 \mathrm{kN}$ hydraulic actuators located vertically at the two opposite ends of composite slim beam, while the axial compressive load was applied at the top of column by a $6000 \mathrm{kN}$ hydraulic actuator. According to the JGJ/T 101-2015 [13], the cyclic loading history consists of both force-controlled and displacement-controlled steps.

A load cell and a displacement transducer (LVDT-1) were used to monitor the applied vertical load $(P)$ and displacement $(U)$ at each end of composite slim beam. Strains in the steel beam flanges, crossing diaphragms, and rebars were monitored through strain gauges.

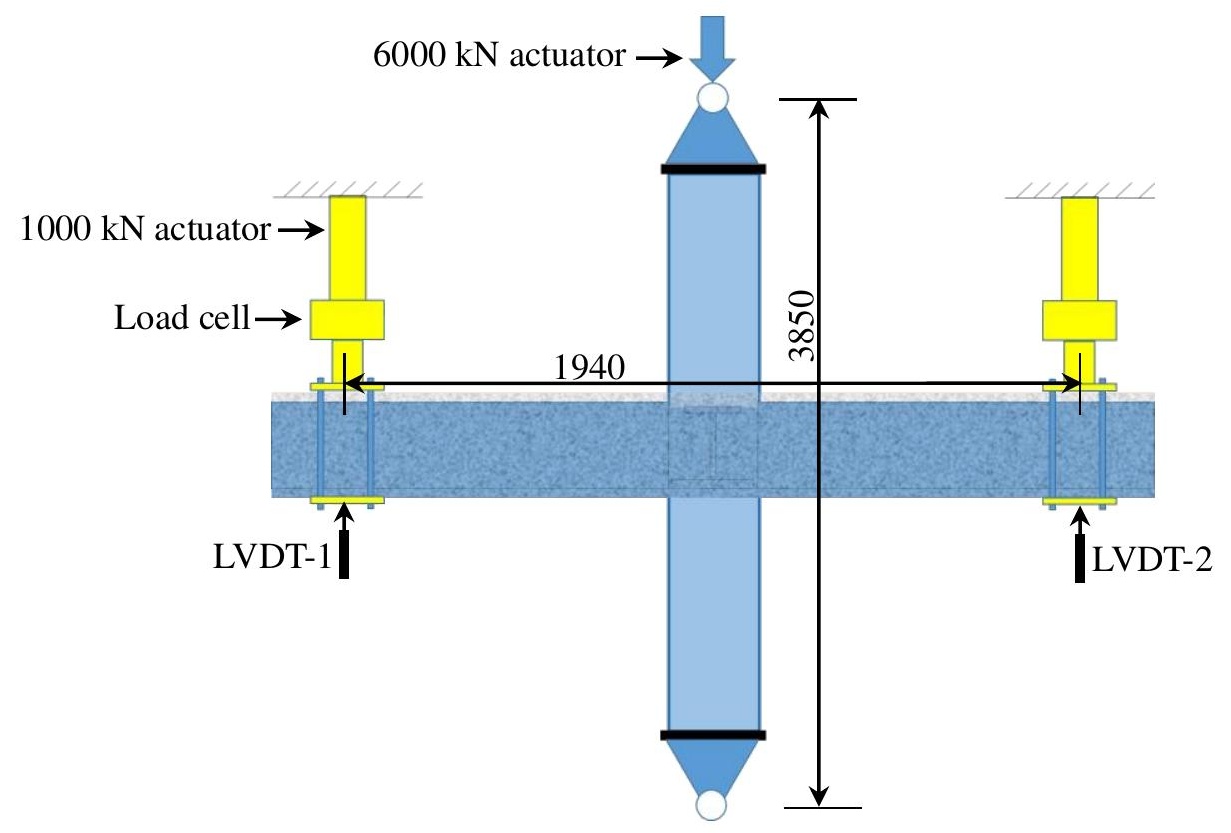

Fig. 6 Test set-up

\section{Failure progression}

(1) Specimen 1

Fig. 7 shows the $P-U$ curves and failure mode of Specimen 1 which has no RC slab. The top steel beam flange began to fracture near the crossing diaphragm with $U=-32 \mathrm{~mm}$ (Fig. 7b), as induced by the stress concentration. The buckling of top steel beam flange was observed at $U=40 \mathrm{~mm}$ (Fig. 7c). At $U=-48 \mathrm{~mm}$, the top steel beam flange completely fractured, followed by the fracture of web (Fig. 7d). 

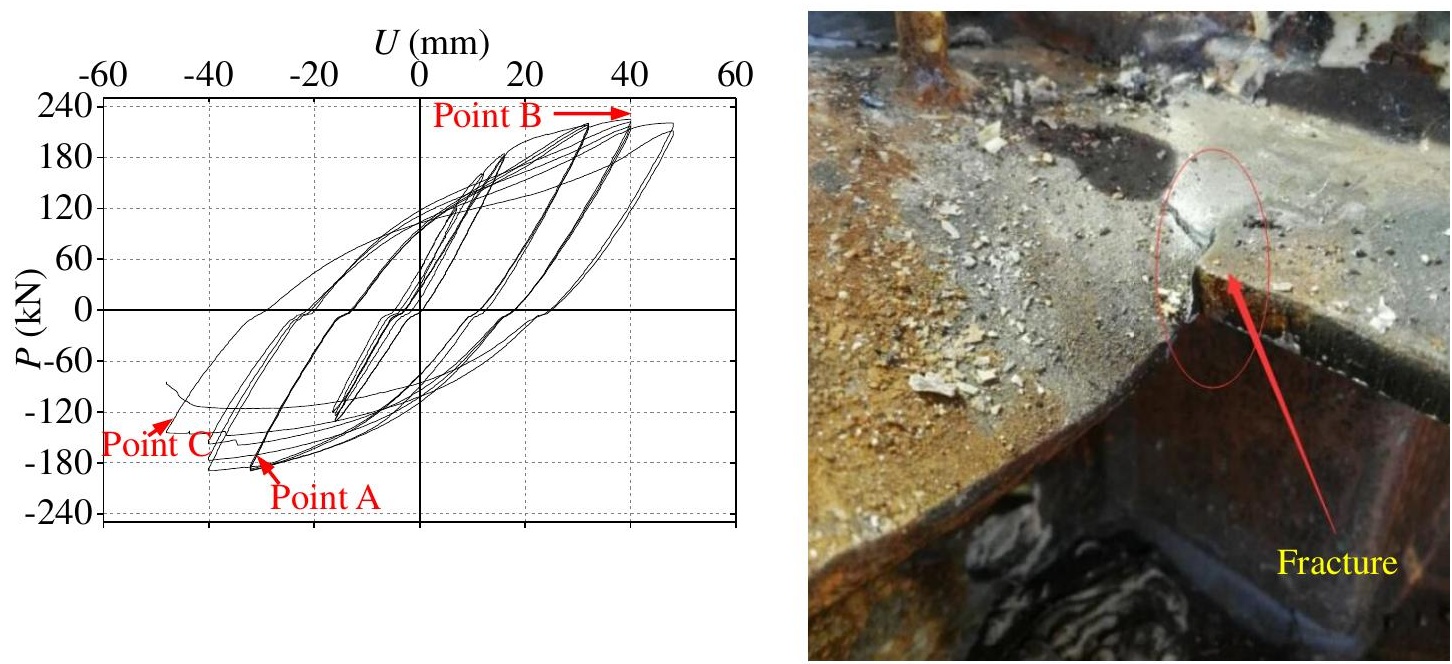

(a) $P$ - $U$ curve

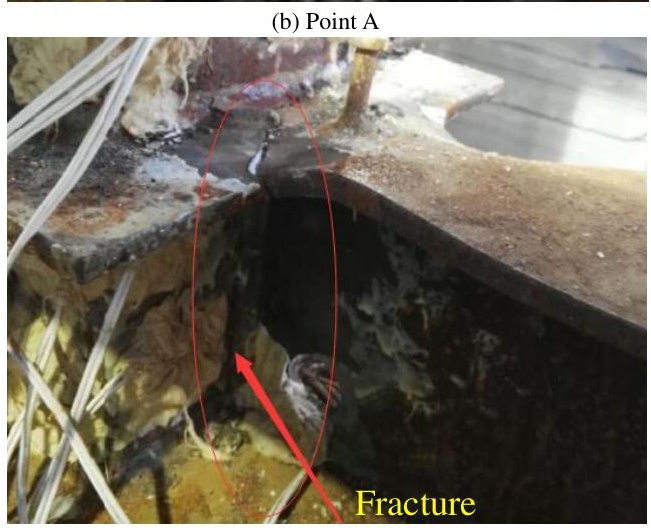

(c) Point B

(d) Point C

Fig. 7 Specimen 1

(2) Specimen 2-4

Since Specimens 2-4 show similar failure modes, Specimen 2 is selected for detailed discussion. The $P-U$ curves and failure modes of Specimen 2 are illustrated in Fig. 8. The bonding failure of rebars occurred first at $U=-30 \mathrm{~mm}$

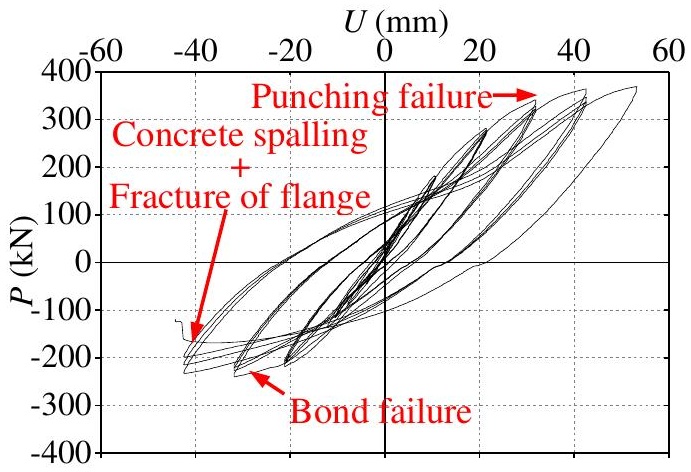

(a) P-U curve

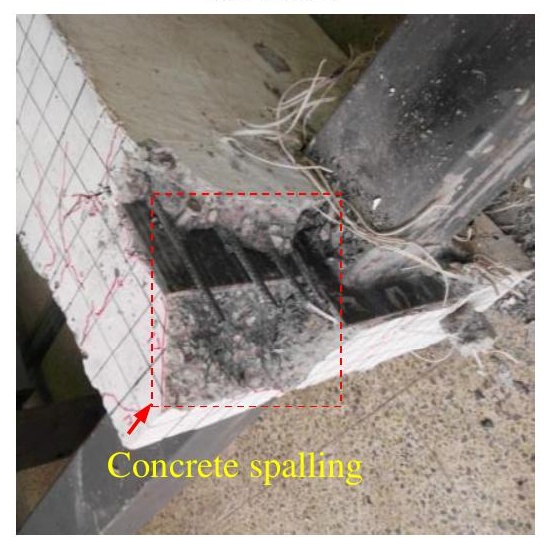

(c) Concrete spalling and the punching failure of concrete was observed at $U=30 \mathrm{~mm}$ (Fig. 8b). At $U=-43 \mathrm{~mm}$, the concrete spalled off (Fig. 8c) and the top steel beam flange eventually fractured completely (Fig. 8d). Similar $P-U$ curves for Specimens 3 and 4 are presented in Fig. 9.

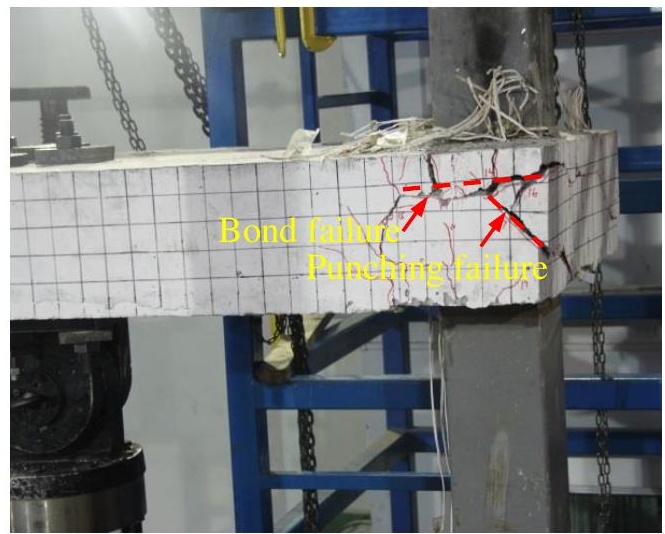

(b) Bonding and punching failures

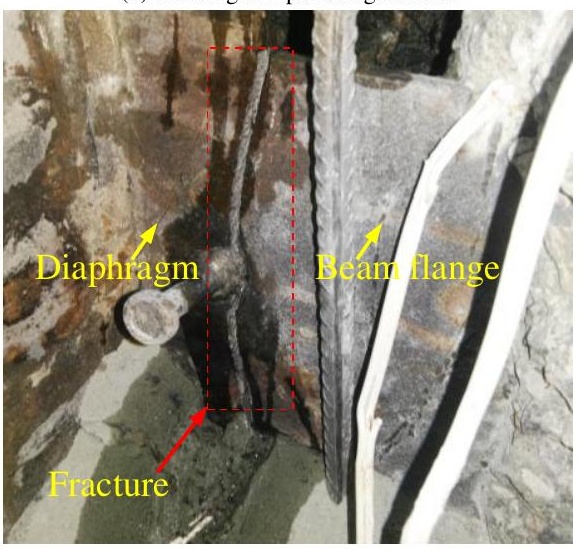

(d) Fracture of flange 


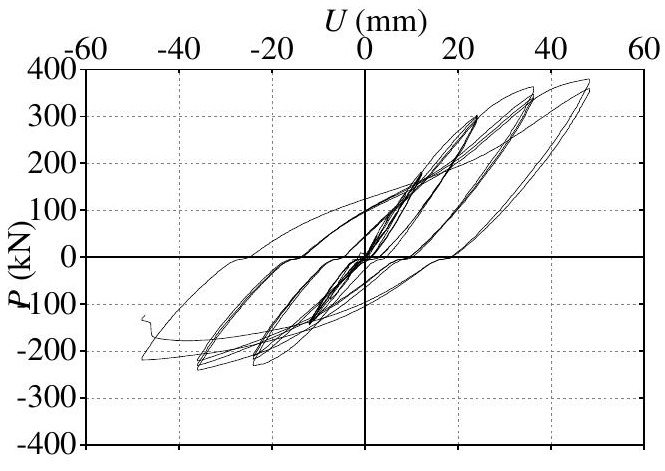

(a) Specimen 3

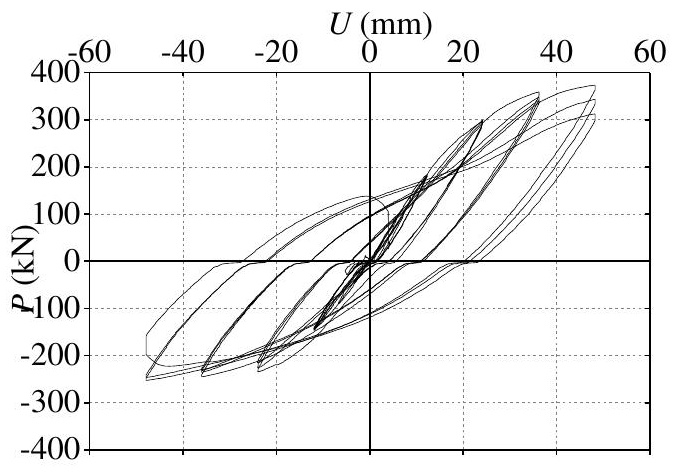

(b) Specimen 4

Fig. $9 P-U$ curves for Specimens 3 and 4

(3) Specimen 5-7

For Specimens 5-7, similar column failure was observed. Specimen 5 is chosen for discussion here. Visible parallel transverse cracks developed first on the top of RC slab adjacent to the column at $U=-9.7 \mathrm{~mm}$ and the cracking region extended with increasing cyclic loads (Fig. 10c). The width of cracks remained small consistently. The wall of steel tube buckled below the diaphragm at $U=57.8 \mathrm{~mm}$ (Fig. 10b). The $P-U$ curves for Specimens 6 and 7 are shown in Fig. 11, which reveal that the tube wall buckles at a smaller $U$ with the higher axial compressive load applied on the column.

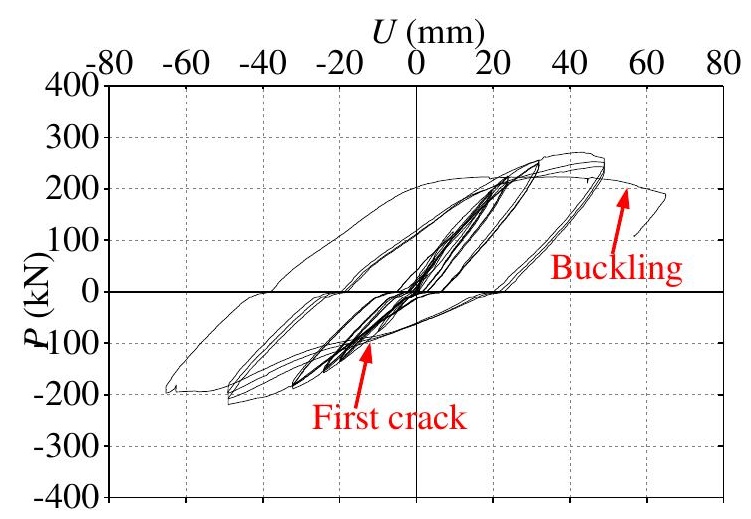

(a) P-U curve

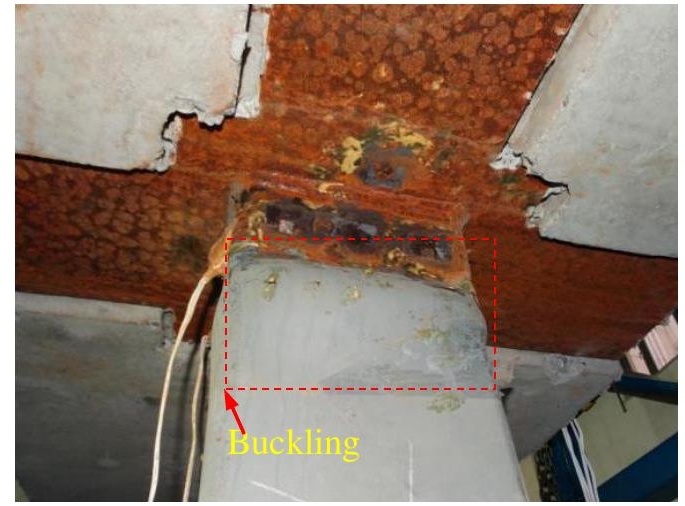

(b) Buckling of steel tube

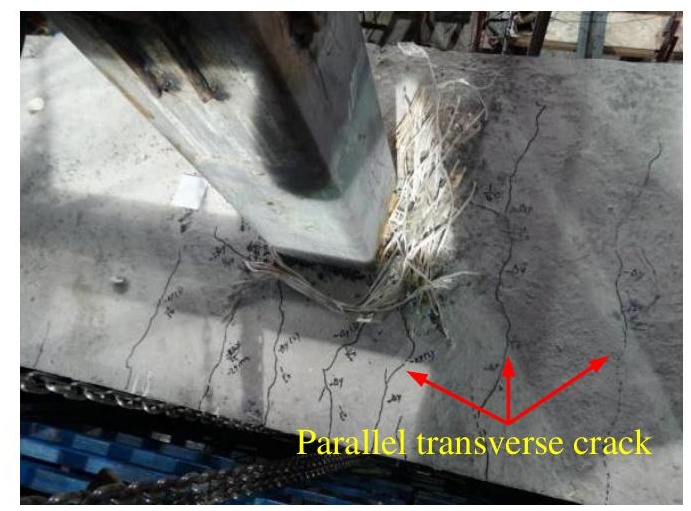

(c) Parallel transverse cracks on the RC slab

Fig. 10 Specimen 5 


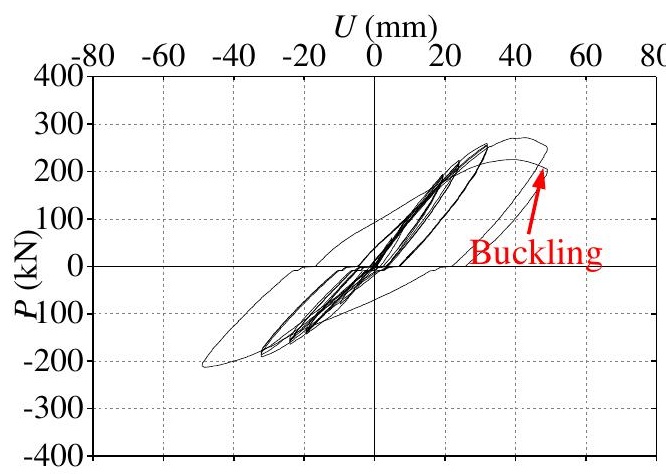

(a) Specimen 6

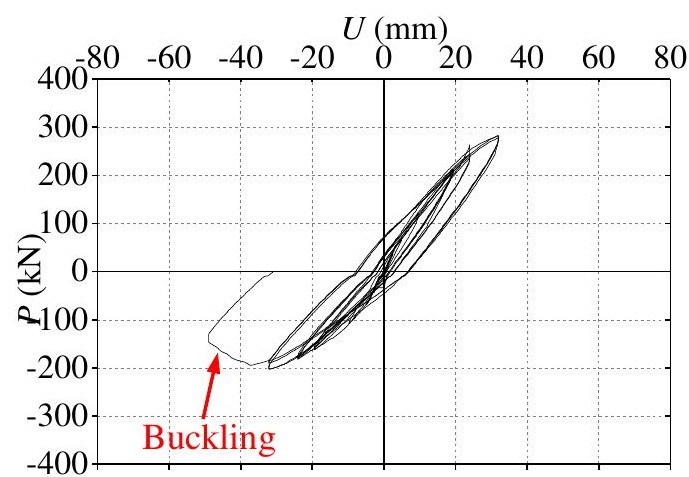

(b) Specimen 7

Fig. $11 P-U$ curves for Specimens 6 and 7

In summary, the failures of Specimens 1-4 are mainly caused by the fracture of top steel beam flange, while the failures of Specimens 5-7 are primarily triggered by the buckling of tube wall. In all tested specimens, the joint zone remains in good shape consistently, which demonstrates that the proposed composite slim floor system inherently meets the principle of strong joint-weak member

\section{Analysis and discussion}

(a) $P-U$ envelope curves

All the $P-U$ envelope curves are highly unsymmetrical, owing to the asymmetric steel beam. Fig. 12 shows the effect of the RC slab on $P-U$ envelope curves. The RC slab embeds the rebars contribute to the beam bending capacity directly and prevents the beam flange from buckling. Under negative loading, the ultimate strength of the specimens with RC slab is $27 \%$ higher than those without RC slab. Under positive loading, the increase is $46 \%$. The results show that the RC slab can significantly increase the bending capacity of a composite slim beam. This fact should be realized in practical rational designs.

Fig. 13 shows the effect of applied axial compressive loads on CFST column on $P-U$ envelope curves. For Specimens 2-4 with beam failure, the axial compressive load has little effect on $P-U$ envelope curves. However, the higher axial compressive load may decrease the deformability of the specimen with column failure.

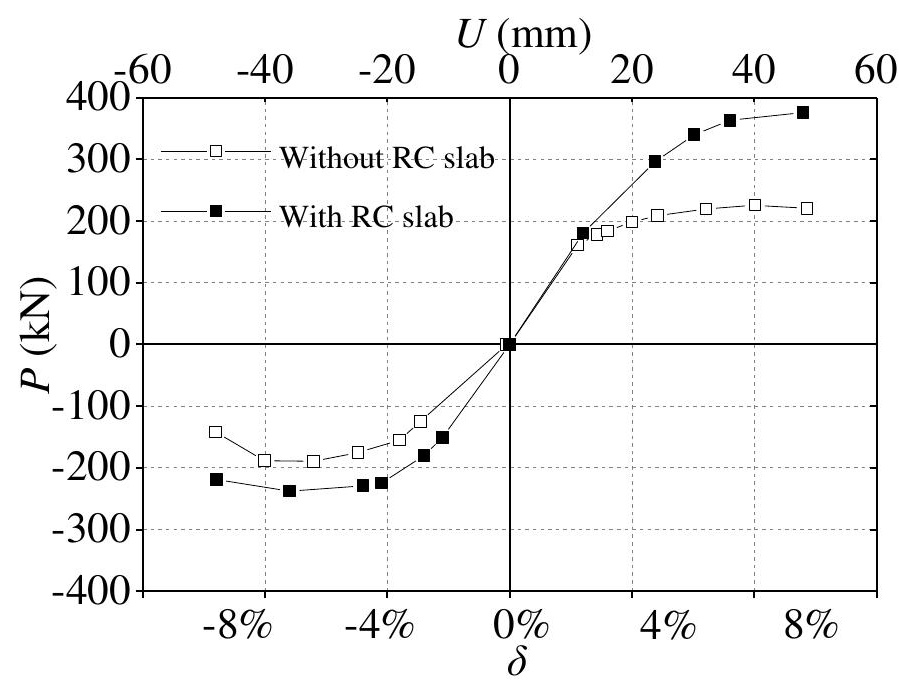

Fig. 12 Effect of RC slab

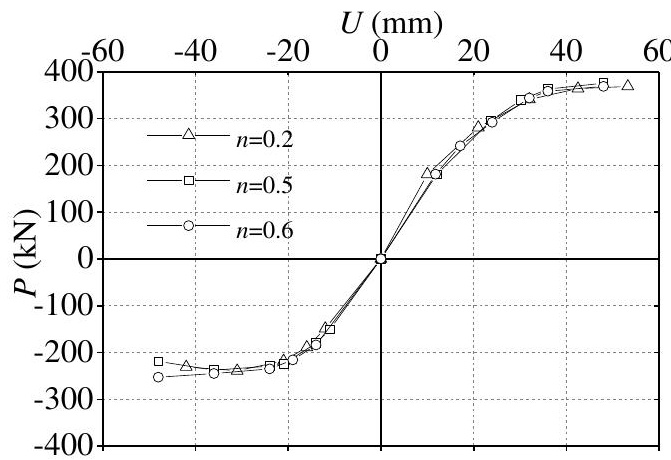

(a) Beam failure

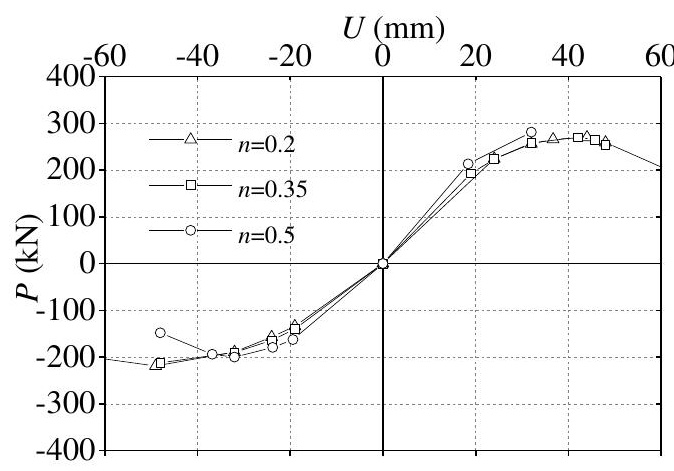

(b) column failure

Fig. 13 Effect of axial compressive loads applied on CFST column

(b) Strains

The readings of the strain gages set on rebars are illustrated in Fig. 14. At exterior joints, the strains of rebars decrease rapidly after $U$ reaches about 24 $\mathrm{mm}$, demonstrating a bond failure of rebars. However, at interior joints the strains of rebars keep increasing before the peak load, indicating the good bond condition of rebars. In practice, a good anchorage should be suggested for the rebars at exterior joints to achieve the fully connection between RC slab and steel beam.

Typical readings of the strain gages set on the steel beam flange and column tube are indicated in Fig. 15. For Specimen 2 with beam failure, the strains of top beam flange exceed the yield strain of steel. By contrary, the strains of column tube are consistently within the yield strain of steel. For Specimen 5 with column failure, the strains of top beam flange do not exceed the yield strain of steel. However, there is a rapid decrease in the strains of column tube, as evidenced by the observed buckling of column tube. 


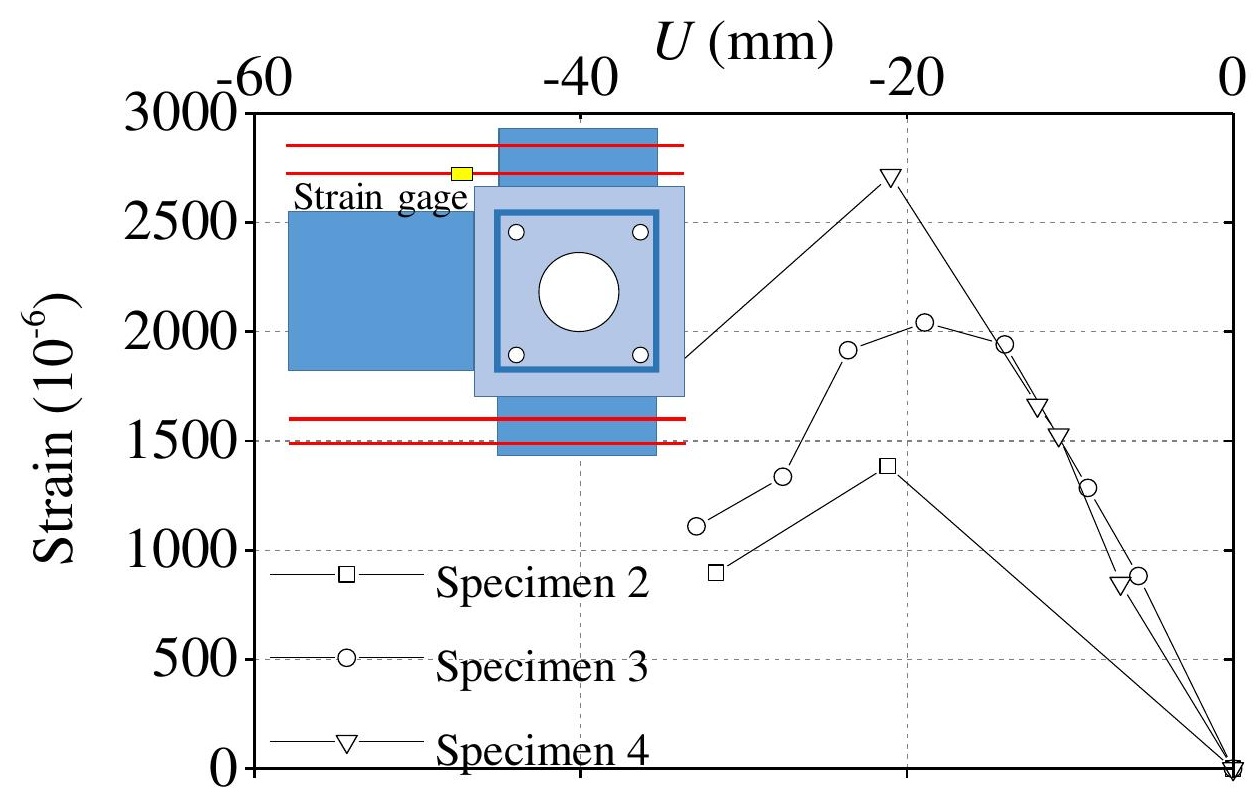

(a) Exterior joints

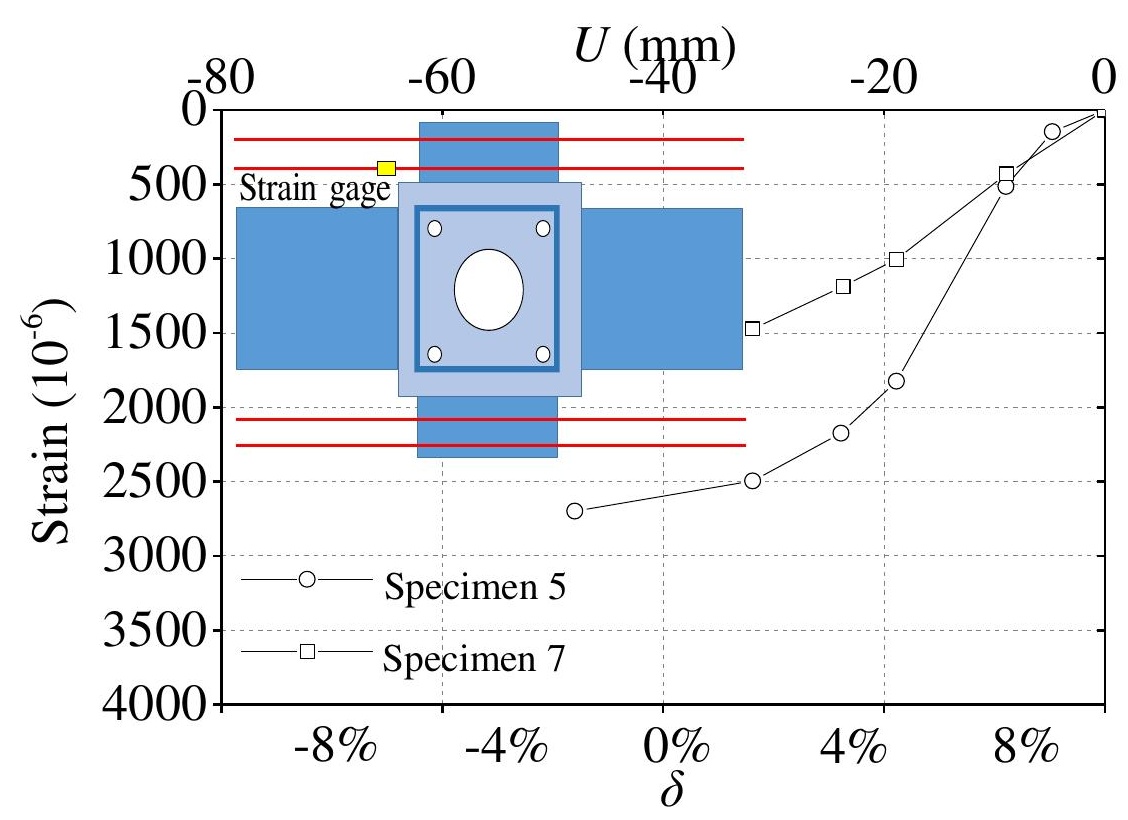

(b) interior joints

Fig. 14 Readings of the strain gages set on rebars

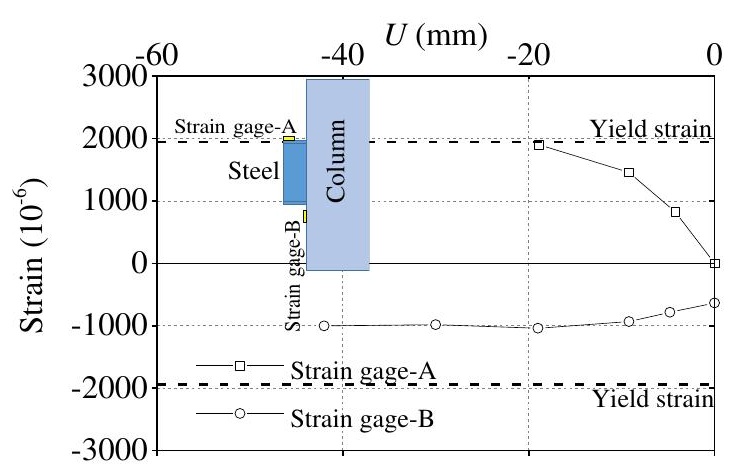

(a) Specimen 2 with beam failure

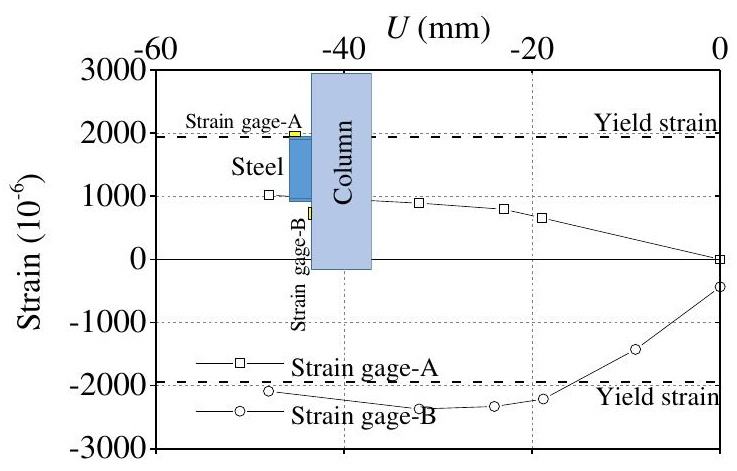

(b) Specimen 5 with column failure

Fig. 15 Readings of the strain gages set on the steel beam flange and column tube 
[14]. The $\mu$ values are listed in Table 3. As seen, the specimens with beam failure have a larger ductility factor, generally exceeding 1.89 . For the specimens with column failure, the $\mu$ value is generally less than 1.81 and decreases with increasing axial compressive loads applied on the CFST column.

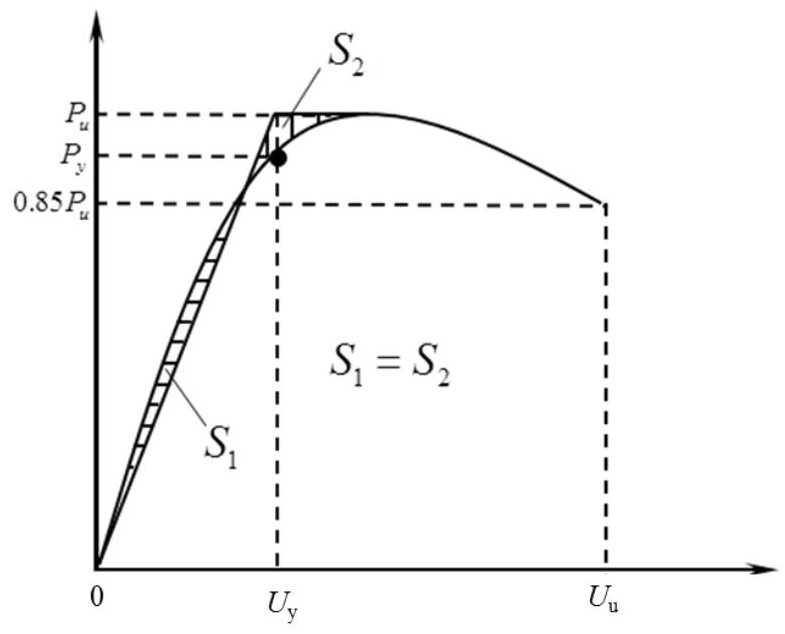

Fig. 16 Method for determining $U_{\mathrm{y}}$ and $U_{\mathrm{u}}$

The story drift $\theta$ is defined as $U /(L / 2)$ where $L$ is the length between composite beam pins, which can be used to evaluate deformation capacity of a specimen. The plastic drift ratio specified in the ASCE/SEI 7-10 [15] and GB $50011-2010[16]$ is $2.0 \%$. The ultimate story drift $\theta_{\mathrm{u}}$, defined as $U_{\mathrm{u}} /(L / 2)$, ranges from 0.044 to 0.056 , which is 2.2-2.8 times of $\theta_{\mathrm{p}}$. Thus, all the specimens satisfy the design code requirements and exhibit good deformation capacity.

Table 3

Test results of all the specimens

\begin{tabular}{|c|c|c|c|c|c|c|c|}
\hline Specimen & Specimen-1 & Specimen-2 & Specimen-3 & Specimen-4 & Specimen-5 & Specimen-6 & Specimen-7 \\
\hline Failure direction & + & + & + & + & + & + & + \\
\hline$P_{\mathrm{y}}(\mathrm{kN})$ & 172 & 218 & 216 & 229 & 249 & 244 & 182 \\
\hline$U_{\mathrm{y}}(\mathrm{mm})$ & 23.9 & 21.2 & 19.6 & 22.6 & 30.3 & 28.9 & 25 \\
\hline$P_{\mathrm{u}}(\mathrm{kN})$ & 160.65 & 1 & I & I & 230 & 1 & 170 \\
\hline$U_{\mathrm{u}}(\mathrm{mm})$ & 44.9 & $>42$ & $>48$ & $>48$ & 54.7 & $>48$ & 42.6 \\
\hline$\mu$ & 1.89 & $>1.98$ & $>2.45$ & $>2.45$ & 1.81 & $>1.66$ & 1.70 \\
\hline$\theta_{\mathrm{u}}$ & 0.046 & $>0.043$ & $>0.049$ & $>0.049$ & 0.056 & $>0.049$ & 0.044 \\
\hline$\theta_{\mathrm{p}}$ & & & & 0.02 & & & \\
\hline$\theta_{\mathrm{u}} / \theta_{\mathrm{p}}$ & 2.3 & $>2.2$ & $>2.5$ & $>2.5$ & 2.8 & $>2.5$ & 2.2 \\
\hline$\xi_{\mathrm{eq}}$ & 0.19 & 0.12 & 0.13 & 0.17 & 0.17 & 1 & 1 \\
\hline Failure mode & Beam failure & Beam failure & Beam failure & Beam failure & Column failure & Column failure & Column failure \\
\hline
\end{tabular}

Note: The "/" represents the number can not be calculated according to the measured data.

\section{Conclusions}

This paper investigates the seismic behavior of biaxial hollow RC slab to CFST column connections, where seven specimens were tested. Based on this study, the following conclusions may be drawn:

1) The proposed composite slim floor system inherently meets the principle of strong joint-weak member, indicating that there is no need to check the shear resistance of joint

2) For specimens with beam failure, the failures are mainly caused by the fracture of top steel beam flange. For specimens with column failure, the failures are primarily triggered by the buckling of tube wall.

3 ) To avoid the bond failure of rebars, a good anchorage should be suggested for the rebars at exterior joints in practical rational designs.

4) The ultimate story drift is 2.2-2.8 times of plastic drift ratio specified in codes and the equivalent damping coefficient for all specimens at peak loads ranges from 0.12 to 0.19 . (d) Energy dissipation

The equivalent damping coefficient $\xi_{\text {eq }}$ is adopted to represent the energy dissipation ability of specimens, which is defined as

$$
\xi_{\mathrm{eq}}=\left(S_{\mathrm{ABC}}+S_{\mathrm{ADC}}\right) /\left[2 \pi\left(S_{\mathrm{OBE}}+S_{\mathrm{ODF}}\right)\right]
$$

where $S_{\mathrm{ABC}}$ is the area enclosed by the curve $\mathrm{ABC}$ shown in Fig. 17. Definitions for $S_{\mathrm{ADC}}, S_{\mathrm{OBE}}$, and $S_{\mathrm{ODF}}$ are similar. The $\xi_{\mathrm{eq}}$ values for all specimens at peak loads are given in Table 3, which range from 0.12 to 0.19 . Since the average $\xi_{\mathrm{eq}}$ is about 0.1 for normal RC joints, the energy dissipation ability of the proposed joints is slightly better than conventional RC joints.

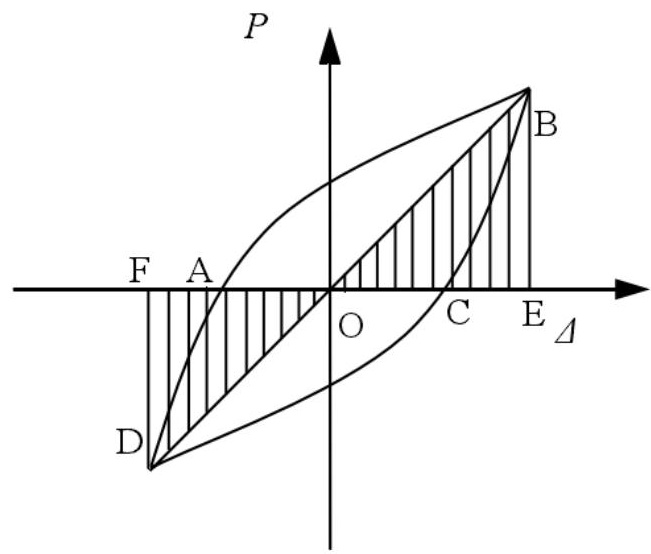

Fig. 17 The calculation of equivalent damping coefficient

\section{Nomenclature}

$A_{\mathrm{c}}$

$A_{\mathrm{s}}$

$f_{\mathrm{cc}}$

$f_{\mathrm{u}}$

$f_{\mathrm{y}}$

$L$

$n$
$N$

$N$

$P$
$P_{\mathrm{u}}$

$P_{\mathrm{u}}$
$P_{\mathrm{y}}$

$P_{\mathrm{y}}$
$U$

$U_{\mathrm{u}}$

$U_{\mathrm{y}}$ the cross-sectional area of infilled concrete the cross-sectional area of steel tube the axial compressive strength of infilled concrete the ultimate strength of stee the yield strength of steel the length between composite beam pins the axial load ratio of CFST column the axial compressive load applied on CFST column the applied vertical load at beam end the vertical peak load the vertical yield load the vertical displacement at beam end the vertical ultimate displacement the vertical yield displacement the story drift 
$\theta_{\mathrm{p}} \quad$ the plastic story drift

$\theta_{\mathrm{u}} \quad$ the ultimate story drift

$\mu \quad$ the ductility factor

$\xi_{\mathrm{eq}} \quad$ the equivalent damping coefficient

\section{Acknowledgements}

The authors greatly appreciate the financial support provided by the National Key R\&D Program of China (No. 2016YFC0701201) and the National Natural Science Foundation of China (No. 51408620).

\section{References}

[1] Mullett D L. Slim floor design and construction[M]. UK: Steel Construction Institute, 1992

[2] Lawson R M, Mullett D L, Rackham J W. Design of asymmetric slimflor beams using deep composite decking[M]. Berkshire: Steel Construction Institute, 1997.

[3] Ryu J, Ju Y K, Yoon S W, Kim S D. Bending capacities of glass fibre reinforced plastic composite slab[J]. Materials Research Innovations, 2013,17(sup2): 12-18.

[4] Silvana, D N, Ana L H C. Composite connections in slim-floor system: An experimental study[J]. Journal of Constructional Steel Research, 2012, 68(1):78-88

[5] Bailey C G. The behaviour of asymmetric slim floor steel beams in fire[J]. Journal of
Constructional Steel Research, 1999, 50(3): 235-257

[6] Ma Z, Mäkeläinen P. Behavior of composite slim floor structures in fire[J]. Journal of Structural Engineering, 2000, 126(7): 830-837.

[7] $\mathrm{Lu} \mathrm{X} \mathrm{H}$, Makelainen P. Strength and stiffness of composite slim floor beams $[\mathrm{C}]$ Proceedings of the 4st Pacific structural steel conference, 1995

[8] Malaska M. Behaviour of a semi-continuous beam-column connection for composite slim floors[M]. Helsinki University of Technology, 2000.

[9] Bernuzzi C, Gadotti F, Zandonini R. Semi-continuity in slim floor steel-concrete composite systems[C]//1st European Conference on Steel Structures, Athens, Greece. 1995.

[10] De Nardin S, El Debs A L H C. Study of partially encased composite beams with innovative position of stud bolts[J]. Journal of Constructional Steel Research, 2009, 65(2): 342-350.

[11] Hosseinpour E, Baharom S, Badaruzzaman W H W, Al Zand A W. Push-out test on the we opening shear connector for a slim-floor steel beam: Experimental and analytical study[J]. Engineering Structures, 2018, 163: 137-152

[12] CECS 159: 2004.Technical specification for structures with concrete-filled rectangular steel tube members; 2004 [in Chinese].

[13] JGJ/T 101-2015. Specification for seismic test of buildings; 2015 [in Chinese].

[14] Park, R. "Ductility evaluation from laboratory and analytical testing." Proceedings of the 9th world conference on earthquake engineering, Tokyo-Kyoto, Japan, 1988.

[15] American Society of Civil Engineers. Minimum design loads for buildings and other structures. Virginia: ASCE/SEI 7-10; 2010.

[16] GB 500011-2010. Code for seismic design of buildings; 2010 [in Chinese]. 


\title{
EXPERIMENTAL STUDY AND THEORETICAL ANALYSIS ON SEISMIC PERFORMANCE OF CASTELLATED BEAM WITH HEXAGONAL HOLES
}

\author{
Lian-Guang Jia *, Ran Bi, Yu-Xiao Lang and Xue-Feng Li \\ School of Civil Engineering, Shenyang Jianzhu University, Shenyang 110168, China. \\ *(Corresponding author: E-mail: syjlg@163.com)
}

\section{A B S T R A C T}

Under a reciprocating load, through an experimental study and finite element analysis, the effect of different arrangements of stiffening ribs on the failure mode, local stability, and hysteretic performance of castellated beams with hexagonal holes is investigated. The test specimens comprise four steel castellated beams, two of which are reinforced with transverse stiffening ribs having the same opening rate but different height-to-thickness ratios of the webs; the two other steel castellated beams do not have any stiffening ribs. The results show that the steel castellated beams with a high height-to-thickness ratio are prone to local buckling failure of the webs. When, under a low-cycle reciprocating load, the transverse stiffening ribs are arranged, and the steel castellated girder is constrained by the plane, the failure mainly occurs at the hole angle position, and the hysteretic performance of the steel castellated girder is obviously improved compared with the non-stiffened steel castellated beam. Using finite element analysis, we realize that different arrangements of transverse stiffening ribs change the failure mode of the steel castellated beam and that the reasonable arrangement of the stiffening ribs has a significant influence on the hysteretic performance of the castellated beam.

A R T I C LE H IS T O RY

$\begin{array}{ll}\text { Received: } & \text { 24 June } 2019 \\ \text { Revised: } & \text { 12 May } 2020 \\ \text { Accepted: } & \text { 30 May } 2020\end{array}$

\section{K E Y W O R D S}

Castellated beam;

Hysteretic performance;

Experimental study;

Transverse stiffening ribs;

Finite element analysis;

Failure mode

\section{Introduction}

The castellated beams are formed by dislocated welding after cutting out certain geometrical patterns from the webs of H-beam members. As a result, the section height of the beams increases, and their flexural stiffness improves significantly. Moreover, the web openings allow the pipeline equipment to pass easily. However, the height-to-thickness ratio of the web is increased, which may lead to the local instability under loading; the seismic performance of the local buckling of the castellated member webs especially may be severely affected by the earthquake. Steel castellated girder webs have a variety of local buckling modes [1] such as the buckling of piers and bridges due to shear, bending, compression, local concentration force, and so on [2]. Therefore, the focus of recent research has been on the strength and local buckling of the perforated webs. To popularize the application of castellated beams, to avoid the structural failure caused by the buckling of the webs, and to meet the requirements of seismic structures, the arrangement of stiffening ribs in the holes of the castellated beams has become an important structural measure in some building structures.

Various scholars have focused on the static performance of the local stability of castellated beams. For example, R. Feng [3] carried out bending tests on 12 specimens and compared the calculated bending strength according to the standards of various countries; they found that the American National Standard and the North American Code are relatively conservative, while the European Code 3 is not so conservative. In another work, Xianhui Li [4] theoretically analyzed the critical buckling capacity of composite beams and proposed a prediction formula for the critical buckling capacity of composite beams and validated the formula by experimental data. Chen D. Y. et al. [5-7] examined the elastic critical buckling load of honeycomb beams and showed that transverse stiffeners could significantly improve their buckling resistance. Besides, Jia L.G. [8-10] studied the shear bearing capacity of honeycomb beams under different conditions and reported that the shear bearing capacity of the beams is greatly affected by flange thickness, opening ratio, and pass type; they also proposed a simplified formula for calculating shear bearing capacity and presented a correlation between bending and shear. Some other relevant works in this context can also be found elsewhere [11-13].

Although researches have mostly focused on the static performance, including the shear and buckling capacity, of honeycomb beams, some works have also analyzed the seismic performance of honeycomb beams and examined the impact of different factors on the hysteretic performance of these beams. For solid web beams, setting up transverse stiffeners increases the dead weight of the beams and wastes steel, so using stiffeners in steel honeycomb beams can be a significant area of research; indeed, little research has been conducted into the subject. Therefore, this paper considers a regular honeycomb beam with hexagonal openings under six regular reciprocal loads as the research object and adopts the method of combining theoretical analysis with experimental research to study the hysteretic behavior of steel honeycomb beams under low-cycle reversed loading; we also investigate the effect of the arrangement of the transverse stiffening ribs on improving the bearing capacity, stiffness, ductility, and energy dissipation of steel honeycomb beams.

\section{Experimental study}

\subsection{Test specimens}

The dislocation welding method was employed to produce the hexagonal castellated beams. The steel castellated beam is loaded in the middle, and to avoid the local buckling failure, two symmetrical transverse stiffeners with the same material as the beam and with a thickness of $14 \mathrm{~mm}$ were welded in the middle. A steel castellated beam with the transverse stiffener led to a thickness of $6 \mathrm{~mm}$ symmetrically in the position of the pier plate. One plate containing six openings was welded to the ends of all the specimens to be fixed to the bearing with bolts. Two groups, each including two specimens, were designed; in the first group, the web thickness is changed to vary the height-to-thickness ratio of the web and to study the destruction form and hysteretic performance of the castellated beams. The second group investigates the effect of setting up transverse stiffeners. The model of the test specimen along with the related dimensions is presented in Fig. 1, and Table 1 lists the specific dimensions of the steel castellated beams.

\subsection{Material performance test}

Q355B grade steel was selected, and its yield strength (fy), ultimate strength (fu), and elastic modulus (E) were measured. The tensile specimens were sampled from the base metal according to the requirements described in ref [14], and two plates with a thickness of 6 and $8 \mathrm{~mm}$ were selected. Three tensile specimens were prepared and analyzed to obtain the average mechanical properties of each sample. The dimensions of the standard tensile specimen are presented in Fig. 2. Then, according to the conditions reported in ref [15], the standard tensile specimen was processed. Using the yield and ultimate loads of the specimen recorded by the tensile machine, the yield strain of the specimen measured by the resistance strain gauge and the cross-sectional area of the actual test specimen. The tensile test results are tabulated in Table 2. 
Cross-sectional dimensions of the castellated beams

\begin{tabular}{cccccc}
\hline & Beam ID & Sectional dimension & Hole height (mm) & Height-to-thickness ratio & Opening ratio $(\%)$ \\
\hline \multirow{2}{*}{1} & FWL-240-85 & $400 \times 200 \times 4.5 \times 8$ & 240 & 240 & 85 \\
& FWL-240-75 & $400 \times 200 \times 5.1 \times 8$ & 240 & 60 \\
6 & JJFWL-240-85 & $400 \times 200 \times 4.5 \times 8$ & 240 & 75 \\
\hline
\end{tabular}

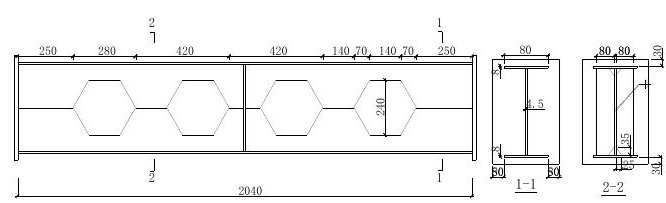

FWL-240-85

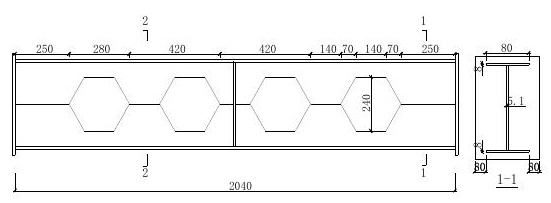

FWL-240-75

(a) Steel castellated beam

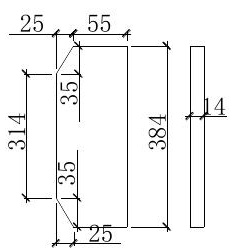

Middle stiffening rib

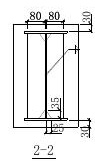

$\underline{2-2}$

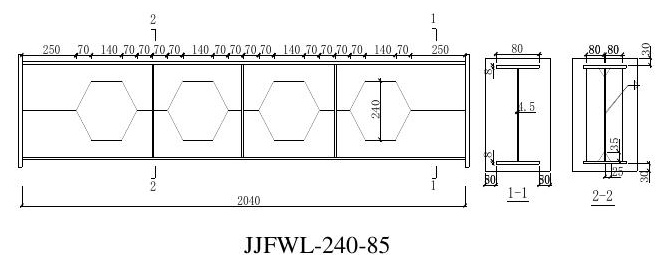

JJFWL-240-85

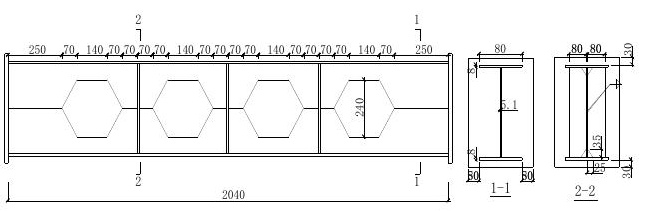

JJFWL-240-75

(b) Steel castellated beam with transverse stiffeners

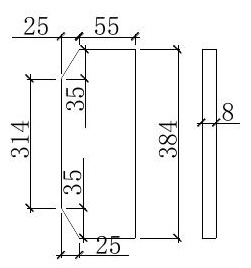

web stiffening rib

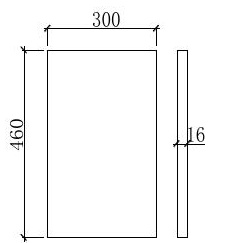

wallboard

(c) Stiffening ribs and end plate

Fig. 1 A sketch map of the castellated beams
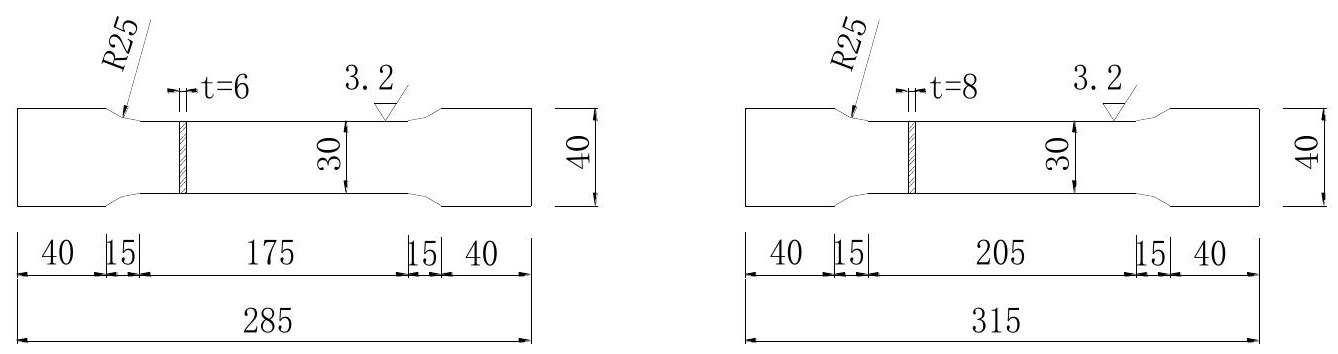

Fig. 2 Standard specimen for tensile testing

Table 2

Mechanical properties of steel

\begin{tabular}{|c|c|c|c|c|}
\hline Specimen specification & Specimen ID & $f_{y}\left(\mathrm{~N} / \mathrm{mm}^{2}\right)$ & $f_{u}\left(\mathrm{~N} / \mathrm{mm}^{2}\right)$ & $E\left(10^{5} \mathrm{~N} / \mathrm{mm}^{2}\right)$ \\
\hline \multirow{4}{*}{$6 \mathrm{~mm}$} & $6-1$ & 368.3 & 521.5 & 2.09 \\
\hline & $6-2$ & 338.5 & 510.5 & 2.03 \\
\hline & $6-3$ & 347.4 & 508.4 & 2.11 \\
\hline & Average value & 351.4 & 513.5 & 2.08 \\
\hline \multirow{4}{*}{$8 \mathrm{~mm}$} & $8-1$ & 368.5 & 544.5 & 1.96 \\
\hline & $8-2$ & 364.6 & 549.6 & 1.93 \\
\hline & $8-3$ & 360.7 & 547.3 & 2.04 \\
\hline & Average value & 364.6 & 547.1 & 1.98 \\
\hline
\end{tabular}

\subsection{Test equipment and loading system}

The experiments were conducted at the Structural Engineering Laboratory of SJZU University using the designed test device schematically shown in Fig. 3 . In order to ensure the stability of the steel castellated beam, two lateral supports are arranged at 1/4 and 3/4 length of the beam. The image of the actual device is displayed in Fig. 4. To ensure that both ends of the beam do not move up and down and that they can be effectively rotated, we designed 
three one-way hinges and a sliding box as a support bearing connection as depicted in Fig. 5. The left end plate of the specimen is connected to the fixed pier by high strength bolts to form the hinge support. The right end plate of the specimen is fixed to the hinge device by high strength bolts and connected with the sliding box to form a sliding hinge bearing. The sliding box uses three rollers on top and bottom and is placed on the groove of the welded plate. A pair of lateral supports are arranged on both sides of the specimen to ensure the stability of the test component in the process of loading.

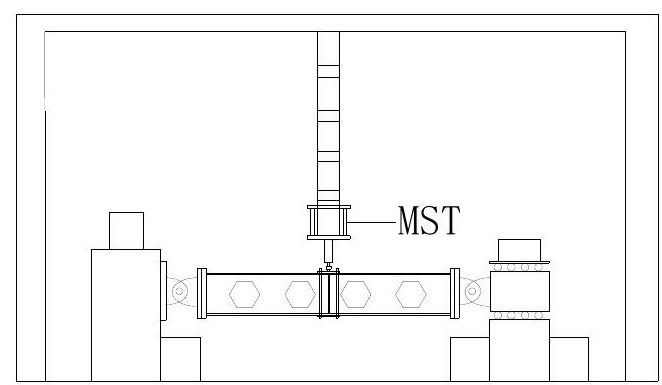

Fig. 3 A sketch of the loading device

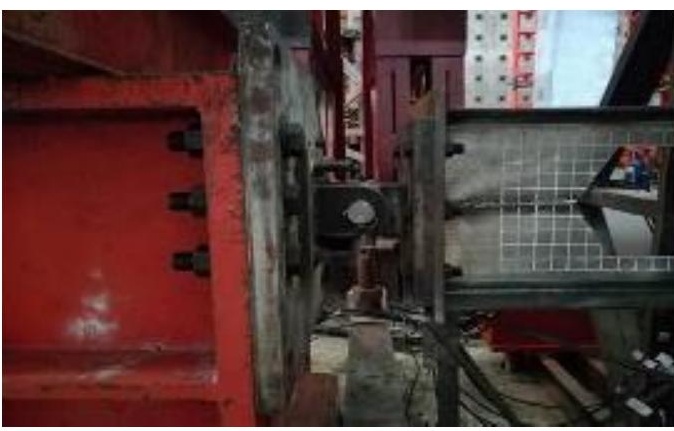

(a) Hinged support

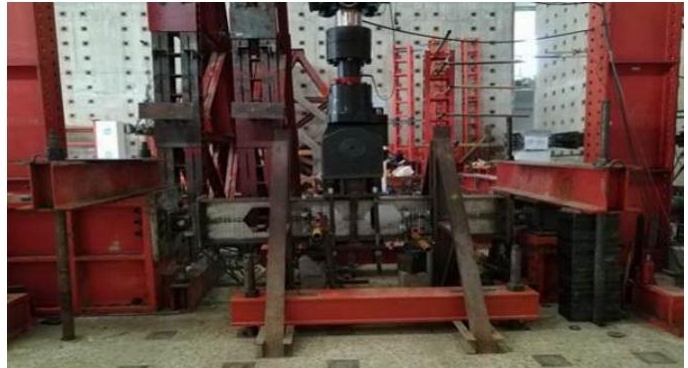

Fig. 4 Test loading device

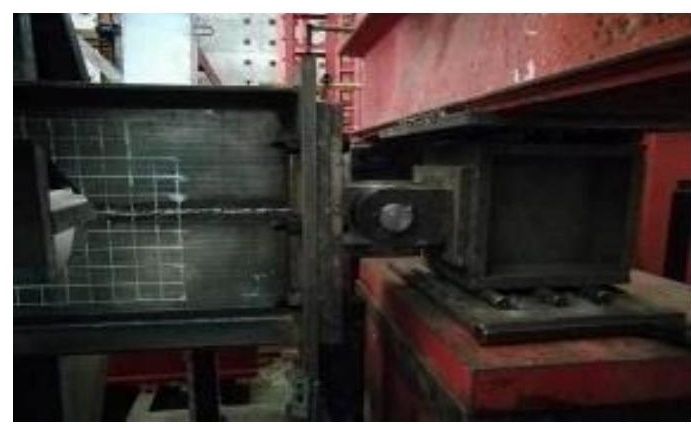

(b) Sliding support

Fig. 5 Support connection device

According to the Specification for Seismic Test of Buildings [16], the load-displacement double-control method is employed for the loading of the quasi-static test. By preloading, we can ensure that the beam suitably contacts the loading point before loading. The stability and reliability of the test equipment is checked, and we ensure that the observation instrument operates normally; then, the initial value of each instrument is recorded. The load-displacement control method is divided into two stages. The beam is loaded by $20 \mathrm{kN}$ in the elastic phase using load control, and each stage is cycled once until the specimen yields. When the beam yields, the displacement control is used to load it, and the yield displacement $\left(\Delta_{\mathrm{y}}\right)$ is increased by $0.5 \Delta_{\mathrm{y}}$; each stage is cycled twice until the specimen is destroyed or the bearing capacity reaches the limit load of $85 \%$ or less. The loading process is shown in Fig. 6 .

During the whole experiment, we observe the deformation and failure of the member and record the order of the damage location and the corresponding loading level. Furthermore, we should ensure that there is no external barrier to the movement of the structural member during the loading process, and there is enough space for the deformation of the beam. The test should be terminated if the plane instability occurs.

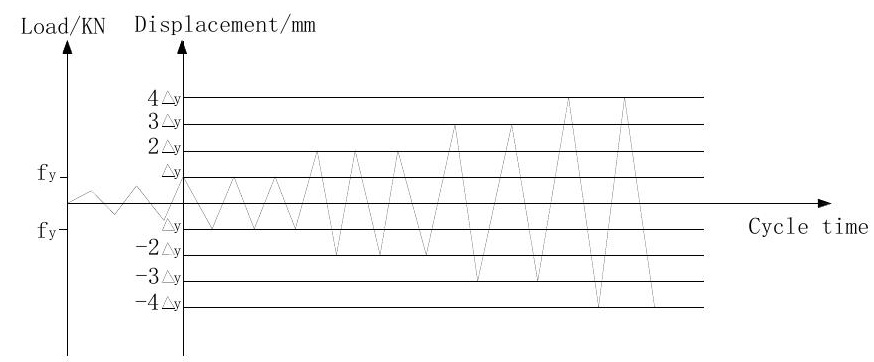

Fig. 6 Loading process of the beam

\subsection{Measurements}

\subsubsection{Displacement measurement}

To study the local buckling deformation of the web of the steel castellated beam, a displacement meter is placed in the web position of the beam to measure the web displacement. It should be noted that in case of using transverse stiffeners, the displacement meter is no longer placed in the web position of the beam. Displacement meters 1,2,3, 4, and 6 are $50 \mathrm{~mm}$, and displacement meter 5 is $100 \mathrm{~mm}$. To calculate the real deflection of the castellated specimen, the effect of beam-end slip must be eliminated by subtracting the cross-middle displacement from the support displacement. The arrangement of the displacement meters and numbering the hole angles are presented in Fig. 7.

\subsubsection{Strain measurement}

To study the influence of the openings on the flange stress, double rows of strain gauges were arranged at the upper and lower positions of the flange. Then, we arranged the strain flowers at the hole angle and the strain gauges in the position of the pier plate to study the variation of the stress in the position between the pier plate and the hole. The layout of the steel castellated beam is shown in Fig. 8.

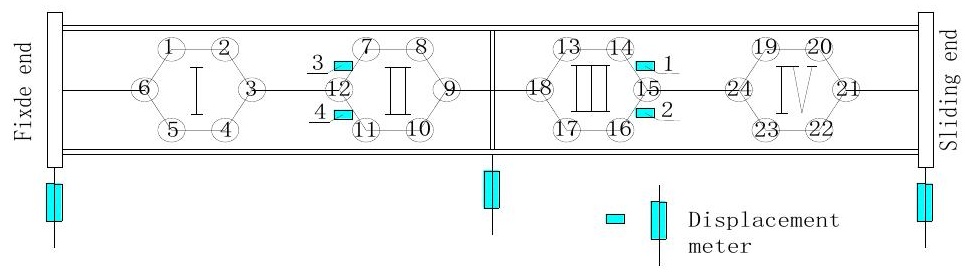

Fig. 7 Arrangement of displacement meters and numbering the hole angles 

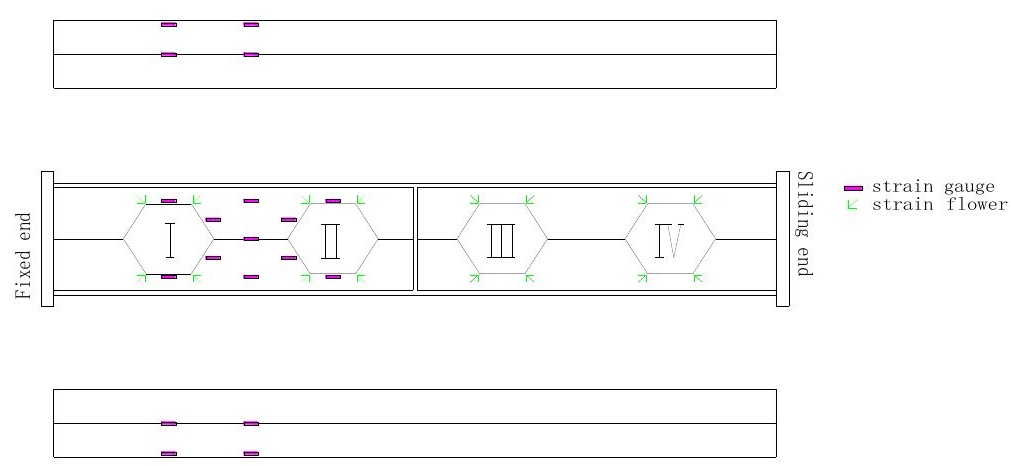

(a) Non-stiffened beam
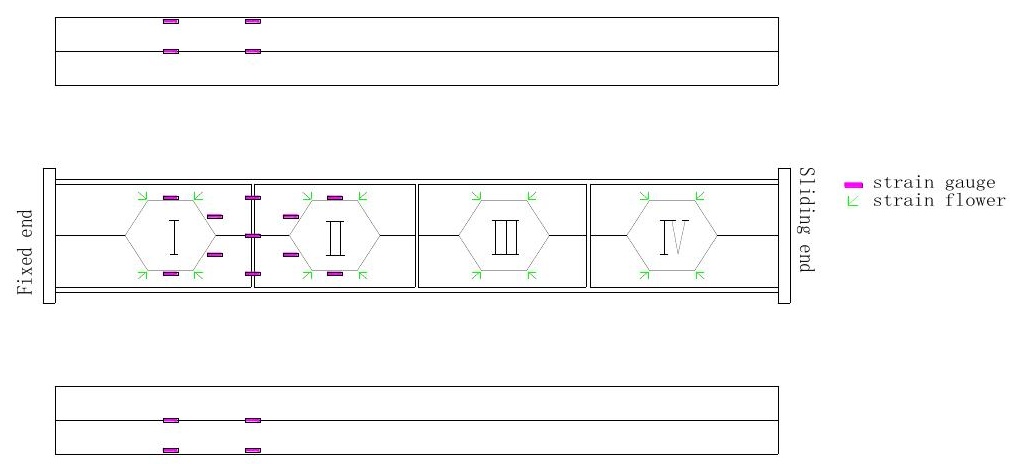

(b) Beam with transverse stiffening ribs

Fig. 8 Arrangement of the strain gauges and strain flowers

\section{Test results}

\subsection{Test phenomena}

According to Fig. 9, the final failure pattern of specimen FWL-240-75 is

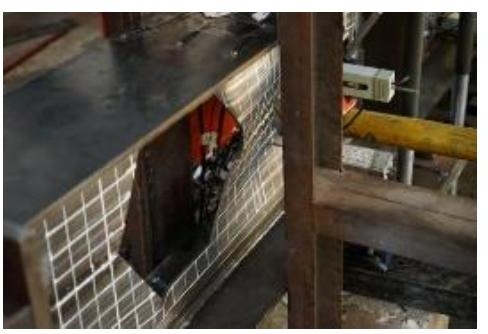

(a) Local buckling of the webs between the holes in the loading process

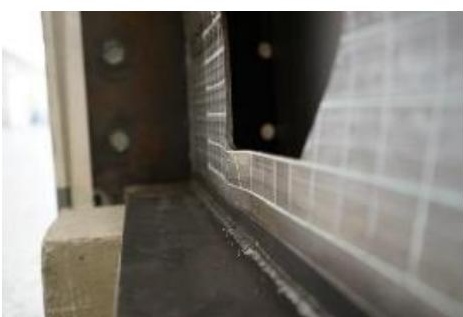

(c) Local buckling of hole angle No. 4 the S-shaped deformation of the webs, and the weld positions do not experience failure. Moreover, no tearing occurs in the hole angle, and the flange does not reach the yield state.

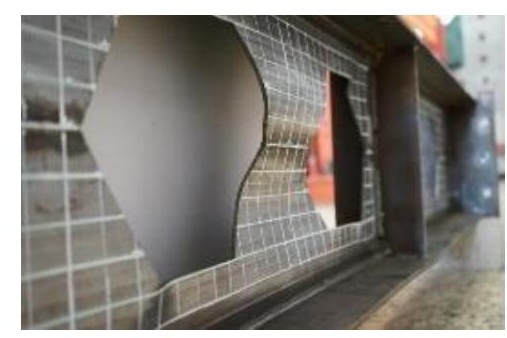

(b) Local buckling of the webs

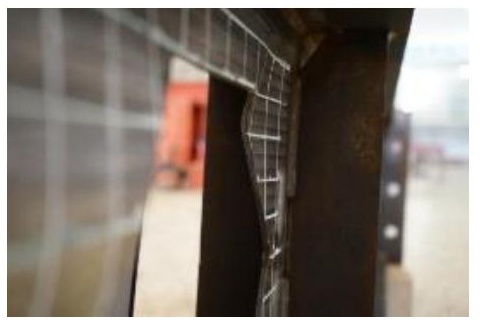

(d) Local buckling of hole angle No. 3

Fig. 9 Failure pattern of specimen FWL-240-75

As can be seen in Fig. 10, specimen FWL-240-85 suffers the local damage to the abdominal plate between the holes. The weld positions do not fail, and no tearing occurs in the hole angle. 


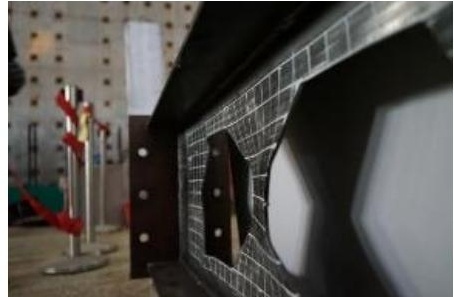

(a) Local buckling of the webs

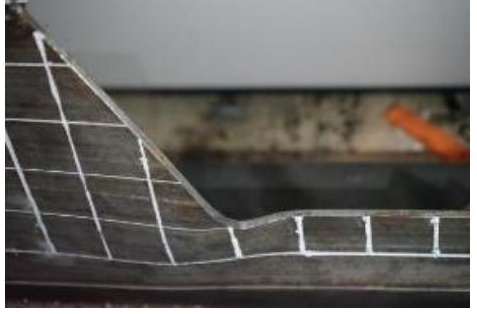

(b) Local buckling of the hole angle

Fig. 10 Failure pattern of specimen FWL-240-85

According to Fig. 11, depicting the final damage pattern of specimen JJFWL-240-75, each hole angle and hole circle experience different degrees of

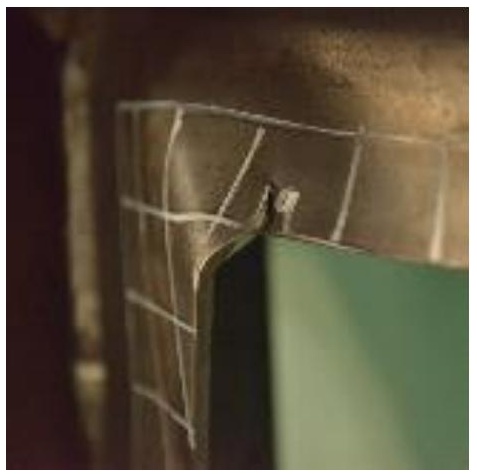

(a) Tearing in hole angle buckling, and hole No. 4 faces the most obvious buckling. Further, the weld positions have not been damaged, and the flange has not reached the yield state.

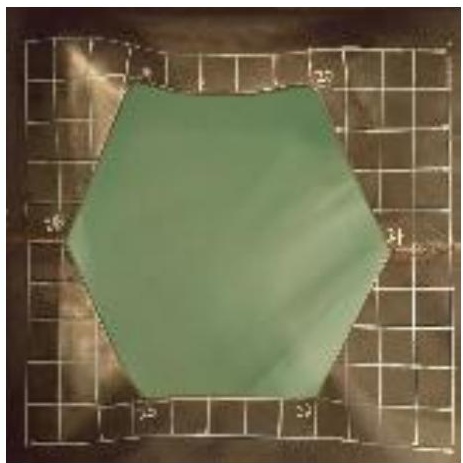

(b) IV failure

Fig. 11 Failure pattern of specimen JJFWL-240-75

Fig. 12. displays the final failure pattern of specimen JJFWL-240-85. It can be seen that different degrees of buckling occur at each hole angle and in the adjacent area. Also, the bearing capacity of the specimen is gradually decreased. When the lower area of I is yielding, the specimen is destroyed.
Damage to holes No. 1 and No. 4 is more serious, and the angle of hole No. 1 is torn, while the weld positions are not destroyed; moreover, the flange does not reach the yield state.

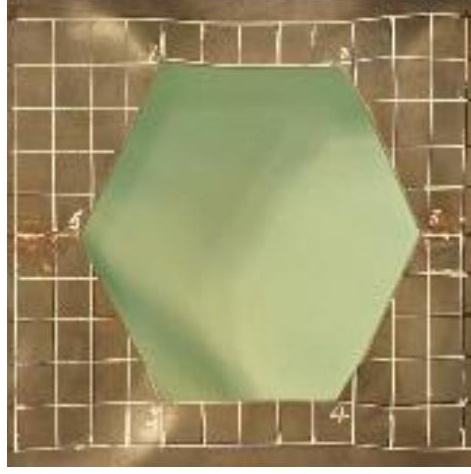

(a) Area near I failure

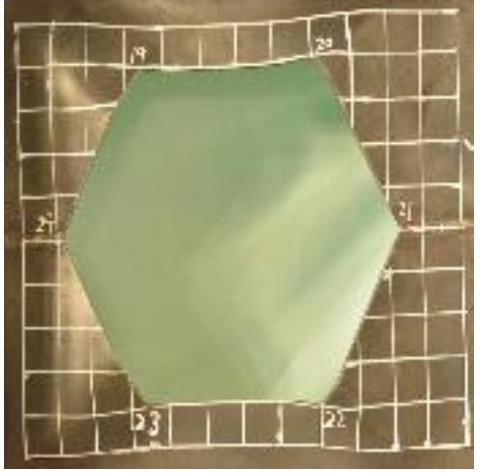

(b) Area near IV failure

Fig. 12 Failure pattern of specimen JJFWL-240-85

Comparing the failure patterns of the above specimens, we can conclude that:

- The main failure mode of specimens JJFWL-240-85 and JJFWL-240-75 is the buckling or tearing of the hole angle, which leads to a decrease in the bearing capacity of the beam. However, specimen JJFWL-240-75 only faces buckling of hole No. 4, but all the four hole angles of specimen JJFWL-240-85 suffer buckling with mutual symmetry.

- No obvious failure occurs between the flange of each specimen and the pier plate between the holes. They are restrained by the stiffening rib, and the pier of the hole no longer buckles. Therefore, the transverse stiffening rib can effectively prevent the pier plate from buckling.

\subsection{Performance analysis}

The hysteresis curves, skeleton curves, and the relations between the corresponding load and displacement of each specimen are presented in Fig. 13, Fig. 14, and Table 3 respectively.

Comparing the hysteresis curves of each specimen in Fig. 13 demonstrates that by setting up the transverse stiffening rib, each member has different degrees of pinch phenomenon; the pinch degree of the hysteresis curves of specimen JJFWL-240-75 is more obvious than that of specimen JJFWL-240-85 because the force applied to each hole of the latter is more uniform in each stage of loading, which leads to the symmetrical buckling of the hole angle. However, later in the loading stage, the hole angle of the end hole of the specimen experiences large buckling deformation, and the end hole is destroyed faster, which leads to a decrease in the energy dissipation ability and deformation ability of the specimen. 


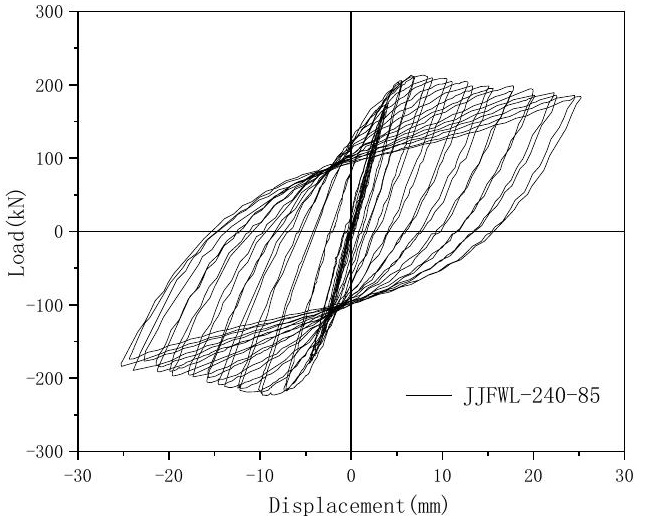

(a) JJFWL-240-75

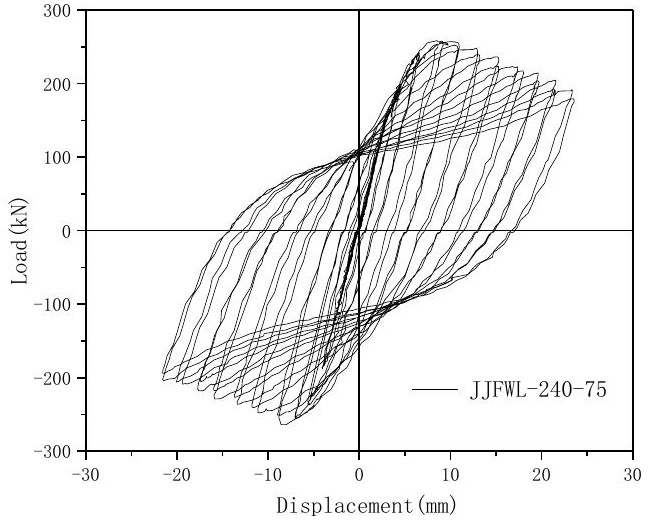

(b) JJFWL-240-85

Fig. 13 Hysteresis curves of the test specimens

The skeleton curves of the specimens in Fig. 14(a) and 14(b) show that the initial stiffness of the beam with transverse stiffening ribs slightly differs from that of the original specimen, and the load capacity and deformation capacity of the stiffened beam are increased. According to Fig. 14(c), the peak load of specimen JJFWL-240-75 is higher than that of specimen JJFWL-240-85; however, after the peak load, the decline in the curve of specimen JJFWL-240-85 is relatively flat, indicating that specimen JJFWL-240-85 has a better deformation ability.

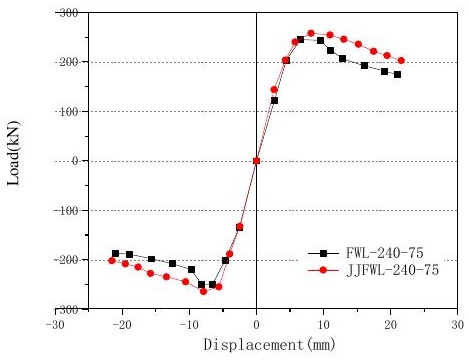

(a)FWL-240-75 and JJFWL-240-75

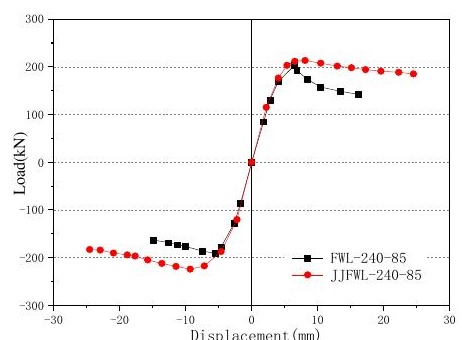

(b)FWL-240-85 and JJFWL-240-85

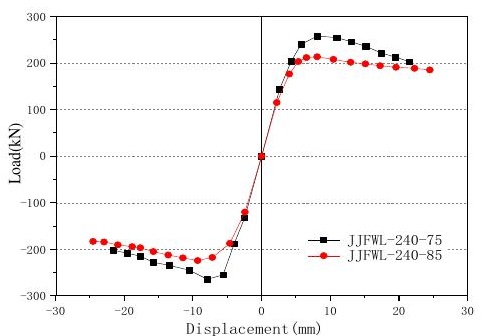

(c)JJFWL-240-75 and JJFWL-240-85

Fig. 14 Skeleton curves of the stiffened and non-stiffened test specimens

According to Table 3, comparing the peak load of the specimens shows that after setting up transverse stiffening ribs, the peak load of the specimen with a height-to-thickness ratio of 85 and the specimen with a height-to-thickness ratio of 75 increases by 8.4 and $2.9 \%$ respectively, which indicates that the transverse stiffener more obviously improves the bearing capacity of the specimen with a high thickness. Additionally, examining the yield displacement and the limit displacement reveals that after setting up the transverse stiffener, the yield displacement and limit displacement of the specimen with a height-to-thickness ratio of 85 improve by 78.14 and $180.18 \%$ respectively, while the yield displacement and limit displacement of the specimen with a height-to-thickness ratio of 75 increase by 38.31 and $64.83 \%$ respectively, which implies that setting up transverse stiffening ribs can greatly enhance the deformation ability of the specimen with a high thickness.

Table 3

The load and displacement of the specimens

\begin{tabular}{|c|c|c|c|c|c|c|}
\hline \multirow{2}{*}{ Beam ID } & \multicolumn{2}{|c|}{ Yield state } & \multicolumn{2}{|c|}{ Peak state } & \multicolumn{2}{|c|}{ Limit state } \\
\hline & $P_{y}$ & $\Delta_{y}$ & $P_{\max }$ & $\Delta_{\max }$ & $P_{u}$ & $\Delta_{u}$ \\
\hline FWL-240-75 & 208.06 & 3.68 & 250.75 & 6.56 & 213.12 & 10.89 \\
\hline FWL-240-85 & 151.79 & 2.79 & 202.19 & 5.73 & 183.54 & 7.72 \\
\hline JJFWL-240-75 & 222.74 & 5.09 & 258.16 & 10.63 & $219 . .44$ & 17.95 \\
\hline JJFWL-240-85 & 194.38 & 4.97 & 219.28 & 9.13 & 186.39 & 20.63 \\
\hline
\end{tabular}

\subsection{Strain analysis}

In this section, the strain variations of the hole angle and flange of the steel castellated girders are analyzed. The section of the specimen with transverse stiffening ribs between the pier plate does not have strain gauges; the location of the measurement points is shown in Fig. 15. 


\begin{tabular}{|lll|}
\hline $\mathrm{A} 1$ & $\mathrm{A3}$ & $\mathrm{A5}$ \\
$\mathrm{A} 2$ & $\mathrm{~A} 4$ & $\mathrm{A6}$ \\
\hline
\end{tabular}

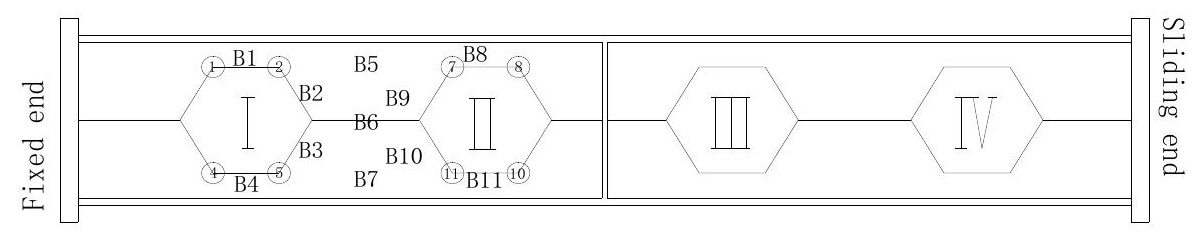

\begin{tabular}{|lll|}
\hline A8 & A10 & A12 \\
\hline A7 & A9 & A11 \\
\hline
\end{tabular}

Fig. 15 Location of the measurement points

\subsubsection{Strain analysis of flange}

All the specimens did not reach the yield state from the beginning of the loading to the destruction of the flange. To study the variation in the different sections of the flange, this section analyzes the strain on each specimen in the yield load, peak load, and the ultimate load states. The changes in the strain on the upper flange of each specimen are shown in Fig. 16.

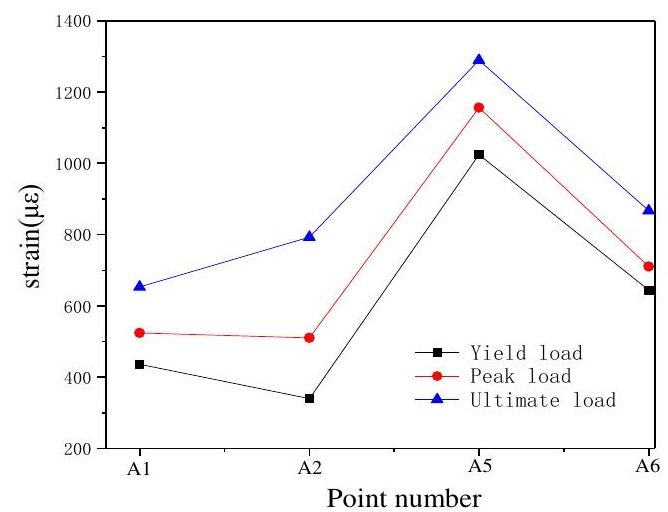

(a) JJFWL-240-75

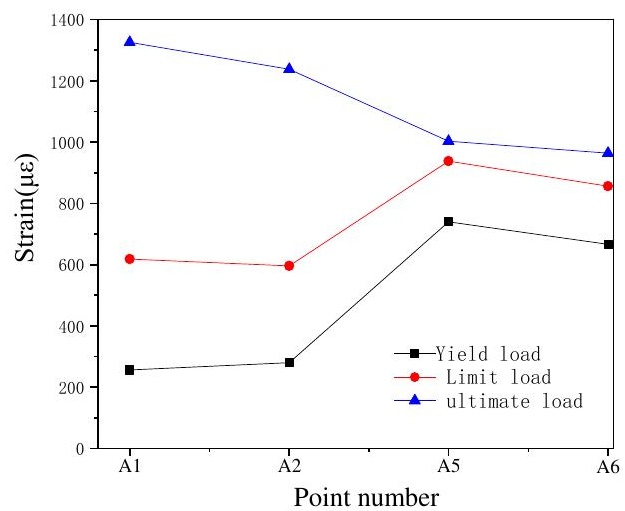

(b) JJFWL-240-85

Fig. 16 The changes of the strain on the upper flange of the test specimens

It is inferred from comparing the variation of the strain on the upper flange of each specimen in different states that when the yield load and peak load are reached, the variation trend in the strain on the flange of the two stiffened specimens with different height-to-thickness ratios is similar. Also, the strain on the flange of specimen JJFWL-240-85 is higher than that of specimen JJFWL-240-75. Nevertheless, at the limit load, the strain on the flange of specimen JJFWL-240-75 is higher than that of specimen JJFWL-240-85 because the buckling failure of the former reaches the limit load, and the stress is transferred from the hole angle to adjacent areas, which causes the shearing ability of the hole section to seriously decrease, and increases the strain on the corresponding flange.

\subsubsection{Strain analysis of steel castellated beams}

To investigate the influence of different height-to-thickness ratios on the strain on the hole angle of steel castellated girders, the strain on each specimen is analyzed by considering two groups of displacement, namely $2 \mathrm{~mm}$ elastic displacement and $6 \mathrm{~mm}$ elastoplastic displacement. The variation of strain at each test point is shown in Fig. 17.

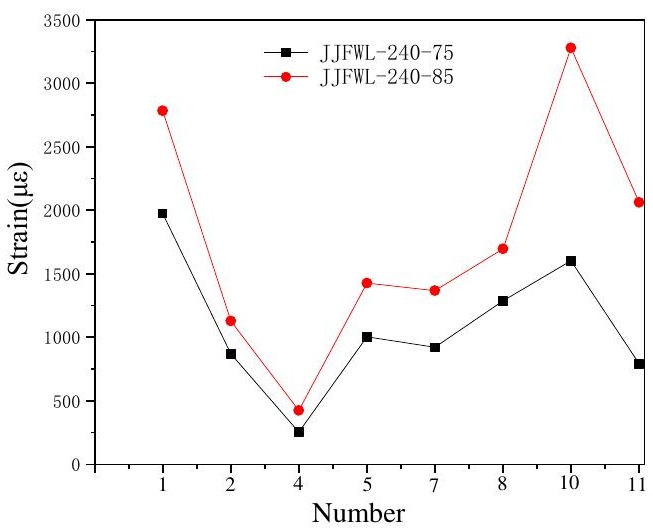

(a) $2 \mathrm{~mm}$ elastic displacement

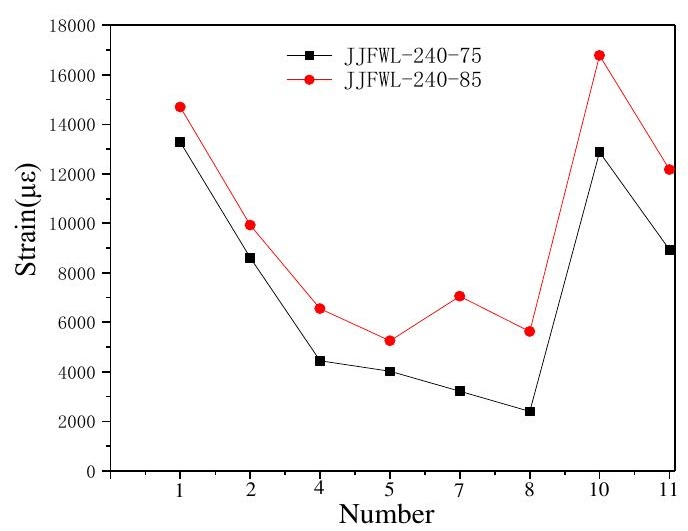

(b) $6 \mathrm{~mm}$ elastoplastic displacement

Fig. 17 The variation in the strain on the hole angle of the stiffened specimens

It can be inferred from Fig. 17 that as the height-to-thickness ratio of the webs increases, the stress concentration on the hole angle becomes more and 
more obvious. When the load displacement is set at $2 \mathrm{~mm}$, hole angles No. 1, 10 , and 11 of specimen JJFWL-240-85 yield, while only hole angle No. 1 of specimen JJFWL-240-75 yields. In the elastic-plastic stage, both hole angle positions reach the yield state.

\section{Establishment and verification of the finite element model (FEM)}

\subsection{Model description}

Global finite element (FE) analysis of the two full-scale castellated beams with stiffening ribs was conducted using ABAQUS 6.11 [17]. The aim of this global study is to compare and validate the model with experimental results and then perform a parametric study. Based on the beam geometry, a skeleton model was initially developed for each of the test specimens. Four-node shell elements (S4R) were used [18] so that the out-of-plane deformation of the honeycomb members can be easily observed under low-cycle reciprocating loads; similar finite element analyses can also be found elsewhere [19].

The built-in elastic-plastic model of ABAQUS, which is mainly utilized to simulate the elastoplastic properties of common metal materials, can be adopted for the steel beam, stiffener, and end plate. Its stress-strain relationship is based on a double broken line model which uses von Mises yield criterion. The model supposes that the flow potential surface function is the same as the yield function and assumes a tension and compression modulus of elasticity similar to the elastic modulus after steel yields, i.e. Es' $=0.01 \mathrm{Es}$, that is, the stress-strain relationship is still linear. The yield strength was based on the results of the material property testing, and the yield strength of steel with a thickness of 6 and $8 \mathrm{~mm}$ was considered to be 351.4 and $364.6 \mathrm{MPa}$ respectively as listed in Table 2 . The Poisson's ratio of the specimen was set at 0.3 , and according to the linear elastic properties of the material, the Young's modulus of steel with a thickness of 6 and $8 \mathrm{~mm}$ was equal to 208 and 198 GPa respectively. The finite element model of the steel castellated beam is illustrated in Fig. 18

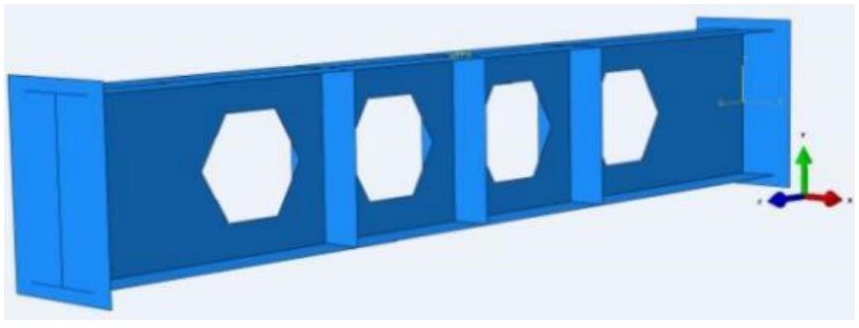

Fig. 18 Finite element model of the castellated beam

\subsection{Model interaction and boundary conditions}

During the processing of the specimen, the connection between the steel honeycomb beam and the stiffening rib is completed by welding, and each part of the member shows good cooperative working performance under the action of a low-cycle reciprocating load; also, the welded part does not show excessive relative movement. Therefore, in the process of ABAQUS modeling, in order to make the model achieve the good overall working performance of the components in actual tests, the "Merge" command in the "Assembly" module is adopted to merge the steel honeycomb beam and stiffener into a whole, and there is no definition of other interaction.

On the left end of the steel castellated beam model, we limit the displacement of $\mathrm{X}, \mathrm{Y}$, and $\mathrm{Z}$ and the rotation around the $Y$-axis and $Z$-axis so as to model the hinge support. On the right end of the beam, we limit $X$ and $Y$ displacement and the rotation around the $Y$-axis and $Z$-axis so as to simulate the sliding support. Additionally, we should limit the displacement of the upper and lower flange along the $X$-axis and the rotation around the $Z$-axis to model the lateral support.

In the process of the mesh division of the finite element model, the whole grid is divided first. To highlight the influence of the holes, the analysis is refined with a smaller meshing technique. The partitioned finite element model is depicted in Fig. 19

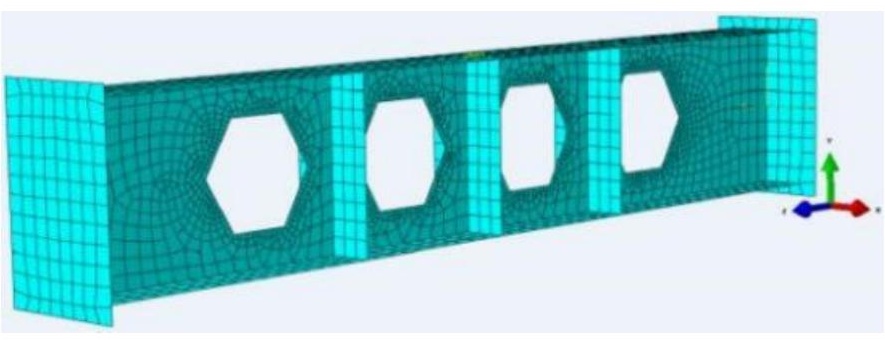

Fig. 19 Mesh division of the finite element model of the castellated beam

\subsection{Parameter verification of finite element model}

The finite element model is established according to the model establishment principle and the size of the specimen used in this experiment. Then, the yield displacement of each specimen is set to $\Delta$, and the method of displacement control loading is employed to load the different models in a low cycle. The loading mode is $0.5 \Delta, 1 \Delta, 1.5 \Delta, 2 \Delta$, etc. Next, the patterns of damage, hysteresis curves, and peak load of each model are obtained and compared with the test results. The accuracy of the model and its applicability to different conditions are verified, which lays the foundation for the simulation of different parameters.

\subsection{Comparison of failure patterns of the beams}

The damage to the hole angle is the failure mode of the specimen with different height-to-thickness ratios and the transverse stiffening ribs; the only difference is that specimen JJFWL-240-75 fails at hole number No. 4, while holes No. 1 and No. 4 of specimen JJFWL-240-85 are destroyed. Comparing the finite element simulation of the steel castellated beam with the experimental results (Fig. 20) reveals a similar failure mode and confirms that the simulation data well match the experimental results.

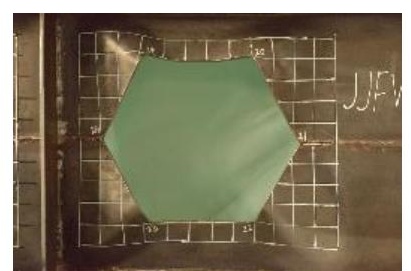

(a) Destruction of JJFWL-240-75

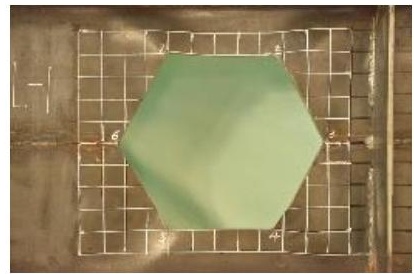

(c) Destruction of the left hole of JJFWL-240-85

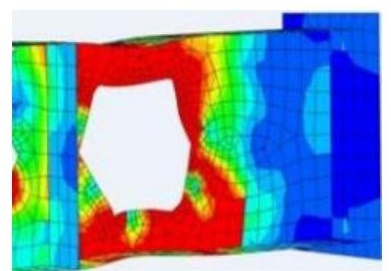

(b) Simulated destruction of JJFWL-240-75

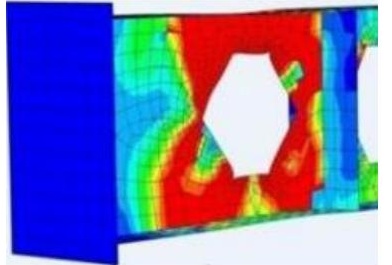

(d) Simulated destruction of the left hole of JJFWL-240-85 


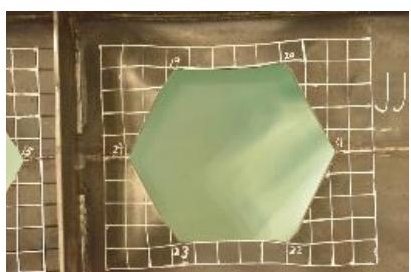

(e) Destruction of the right hole of JJFWL-240-85

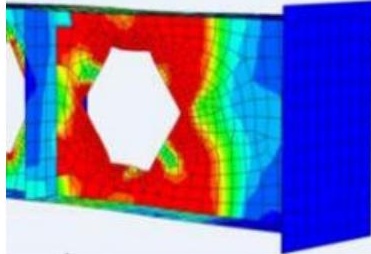

(f) Simulated destruction of the right hole of JJFWL-240-85

Fig. 20 Comparison of finite element data with the experimental results

\subsection{Comparison of hysteresis curves of the beams}

Fig. 21 presents the comparison between the experimental and simulated hysteresis curves of the castellated members. It can be seen that the finite element simulation is in good agreement with the experimentation. In initial loading, the hysteresis curves of the test member are extraordinarily full However, with an increase in the displacement load, the hysteresis curves display a pinch phenomenon because of the buckling of the pier plate and the failure of the hole angle.

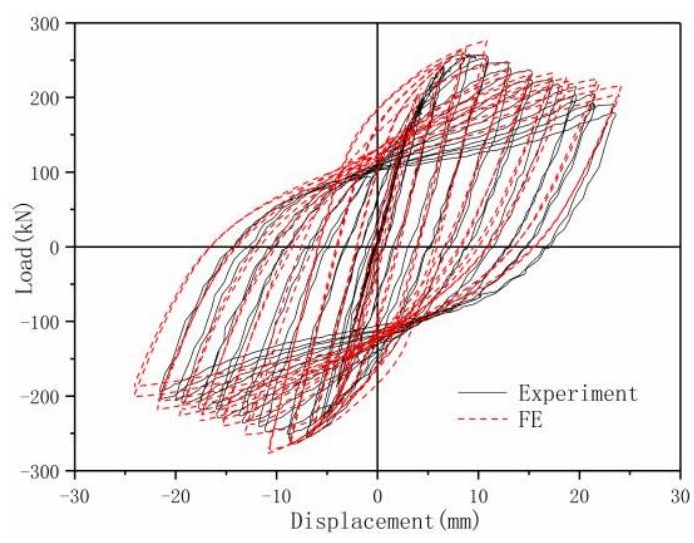

(a)JJFWL-240-75

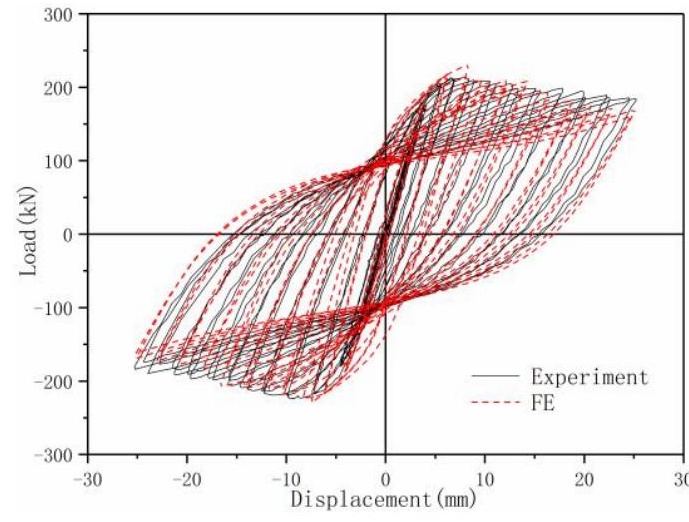

(b)JJFWL-240-85

Fig. 21 Comparison between the experimental and simulated hysteresis curves

\subsection{Comparison of the bearing capacity of the beams}

Table 4 shows that there is a slight difference of $6 \%$ between the experimental results and the finite element simulation data, which denotes that the finite element analysis is consistent with the experimental testing, so it can be used to perform a detailed study on the beams. In fact, by comparing the simulation results with the failure morphology, it is found that the data obtained from the finite element model well match the test results with a minor error. Thus, it can be concluded that the model established in this work is accurate enough to be utilized to further study the local stability and hysteretic performance of steel castellated girders under different conditions.

Table 4

Comparison of simulated and experimental ultimate bearing capacity of the beams

\begin{tabular}{ccccc}
\hline Beam ID & Experimental & $(\mathrm{kN})$ & Simulated & $(\mathrm{kN})$ \\
\hline JJFWL-240-75 & 258.16 & 273.63 \\
JJFWL-240-85 & 219.28 & 5.99 \\
4.15 & 228.38 \\
\hline
\end{tabular}

\section{FE analysis of the influence of different arrangements of stiffening} ribs on the hysteretic behavior of steel castellated beams

\subsection{Design of steel castellated beam with different arrangements of stiffeners}

Using the above finite element model, we set up different arrangements of stiffening ribs; the design parameters of the specimens are listed in Table 5. The section dimensions of the simulation member are the same as that of the test specimens, i.e. $400 \times 200 \times 5.1 \times 8$, and only the stiffener placement changes in the simulation. The other practical parameters are as follows: The height-to-thickness ratio of the web is 75 .

- The opening rate is $60 \%$.

- There are eight opened positive hexagonal holes.

- The beam length is $4200 \mathrm{~mm}$.

- The cross-medium stiffener thickness is $14 \mathrm{~mm}$.

- The transverse stiffener thickness is $6 \mathrm{~mm}$.

Specimen coding is done based on the component name and the stiffener arrangement position; taking JJFWL-2 as an example, "JJFWL" means steel castellated beam with transverse stiffener and " 2 " indicates the component sequence number. In the case of the stiffener layout positions, for example,
" 3113 " as the arrangement position code of the stiffening ribs denotes that eight honeycomb holes are separated by stiffening ribs according to three holes, one hole, one hole, and three holes.

\section{Table 5}

Design parameters of the arrangement of the stiffening ribs in the specimens

\begin{tabular}{ccccc}
\hline Beam ID & $\begin{array}{c}\text { Stiffening rib } \\
\text { position }\end{array}$ & $\begin{array}{c}\text { Opening } \\
\text { Number }\end{array}$ & $\begin{array}{c}\text { Opening rate } \\
(\%)\end{array}$ & $\begin{array}{c}\text { Web } \\
\text { height-to-thic } \\
\text { kness ratio }\end{array}$ \\
\hline JJFWL-1 & 44 & 8 & 60 & 75 \\
JJFWL-2 & 3113 & 8 & 60 & 75 \\
JJFWL-3 & 2222 & 8 & 60 & 75 \\
JJFWL-4 & 211112 & 8 & 60 & 75 \\
JJFWL-5 & 121121 & 8 & 60 & 75 \\
JJFWL-6 & 1331 & 8 & 60 & 75 \\
JJFWL-7 & 112211 & 8 & 60 & 75 \\
JJFWL-8 & 11111111 & 8 & 60 & 75 \\
\hline
\end{tabular}


5.2 Monotonic static loading analysis of steel castellated beams with different arrangements of stiffener

The load-displacement curves of the members are obtained by the monotonic static loading of the steel castellated beam with different arrangements of the stiffening ribs, as shown in Fig. 22. The yield displacement and yield load of the members are also listed in Table 6 .

According to Table 6 and Fig. 22, the yield displacement and yield load of the members are not very different. The initial stiffness difference is rather small, but the peak load, peak displacement, limit load, and limit displacement of the stiffened components obviously differ. After setting up the stiffener in different positions, the sequence of the local buckling of each member changes, so the load capacity of each component is different.

Table 6

Yield load and yield displacement under monotonic static loading

\begin{tabular}{ccc}
\hline Beam ID & Yield displacement $(\mathrm{mm})$ & Yield load $(\mathrm{kN})$ \\
\hline JJFWL-1 & 9.62 & 201.62 \\
JJFWL-2 & 10.11 & 210.38 \\
JJFWL-3 & 10.32 & 213.44 \\
JJFWL-4 & 10.51 & 218.62 \\
JJFWL-5 & 10.69 & 219.13 \\
JJFWL-6 & 11.12 & 221.75 \\
JJFWL-7 & 11.91 & 224.28 \\
JJFWL-8 & 12.31 & 226.67 \\
\hline
\end{tabular}

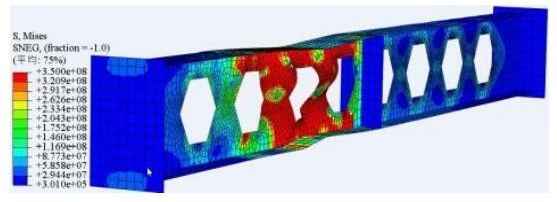

(a) JJFWL-1

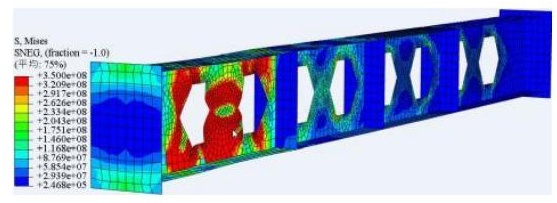

(c) JJFWL-3

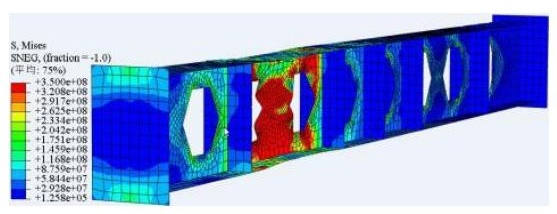

(e) JJFWL-5

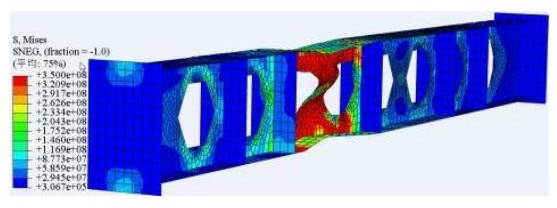

(g) JJFWL-7

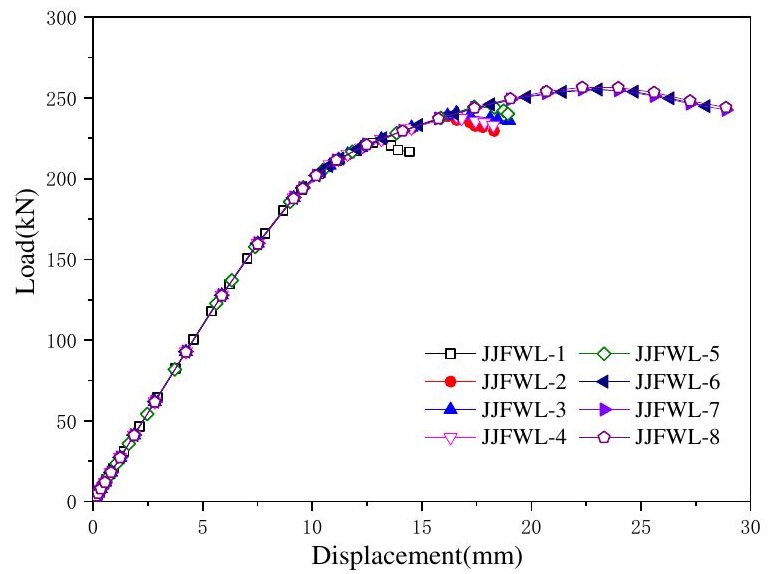

Fig. 22 Load-displacement relationship of the specimens

5.3. Hysteretic behavior analysis of steel castellated beams with different arrangements of stiffener

\subsubsection{Failure mode}

The failure patterns of the steel castellated beams with different arrangements of the stiffening ribs are illustrated in Fig. 23. Analyzing the simulation results of each component reveals that the S-shaped buckling occurs between the piers of specimens JJFWL-1, JJFWL-2, JJFWL-6, and JJFWL-7. However, specimens JJFWL-3, JJFWL-4, and JJFWL-5 fail due to the lateral torsion buckling of the holes between the piers and plates. Specimen JJFWL-8 suffers buckling damage to the flange and hole angle near the loading point, which indicates that the position of the stiffening rib is different, and the damage form or position of each member changes. It can be seen from the failure mode of each component that the local buckling mainly occurs in the area where the pier plate does not have a transverse stiffener. For specimen JJFWL-8, because the pier plate is arranged at the stiffener position, it does not buckle. Furthermore, the hole angle near the loading position first enters the yield state due to bearing the large bending moment and shear force, and then the strain is extended to the flange gradually. At the end, the hole section position forms a plastic hinge, and the specimen is destroyed.

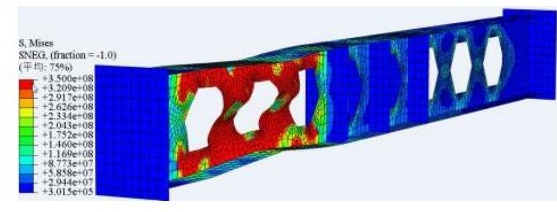

(b) JJFWL-2

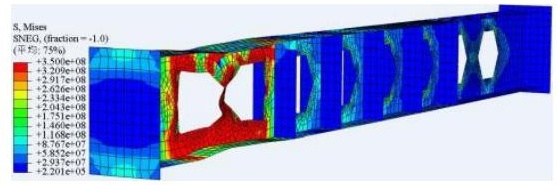

(d) JJFWL-4

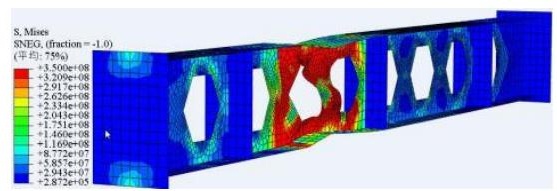

(f) JJFWL-6

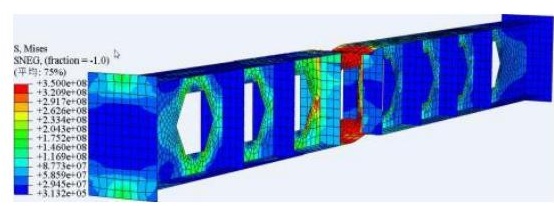

(h) JJFWL-8

Fig. 23 Stress cloud of the castellated beams with different arrangements of stiffeners 


\subsubsection{Hysteresis curves}

Fig. 24 delineates the hysteresis curves of the steel castellated beams with different arrangements of the stiffening ribs. Comparing the hysteresis curves of the members indicates that the hysteresis curves of the members are full regardless of the change in the position of the stiffeners, but the shrinkage

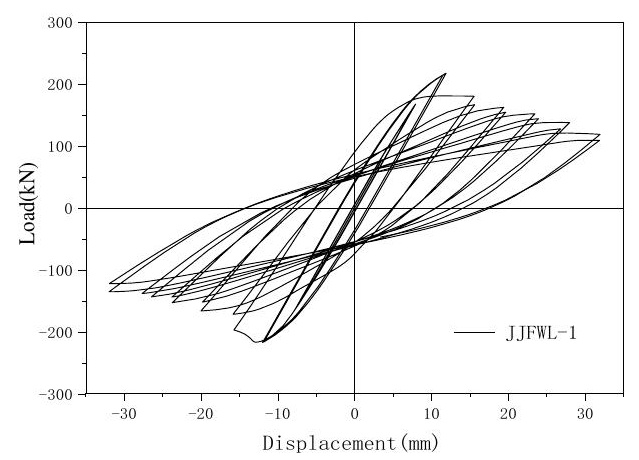

(a) JJFWL-1

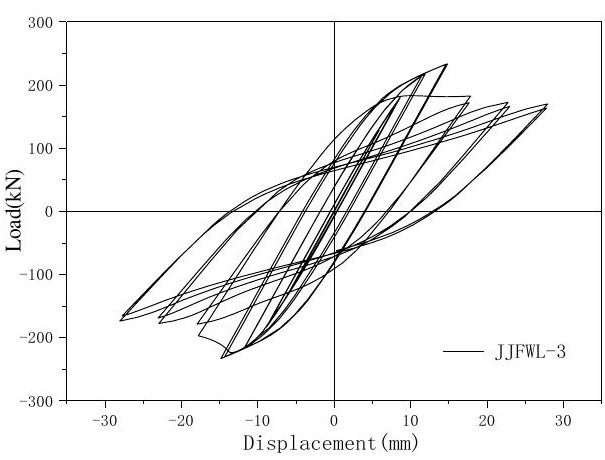

(c) JJFWL-3

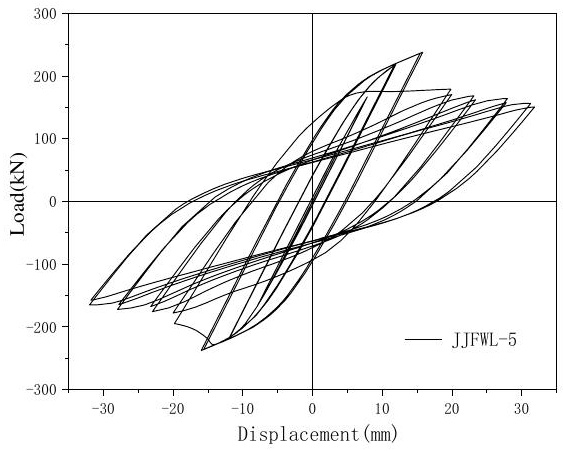

(e) JJFWL-5

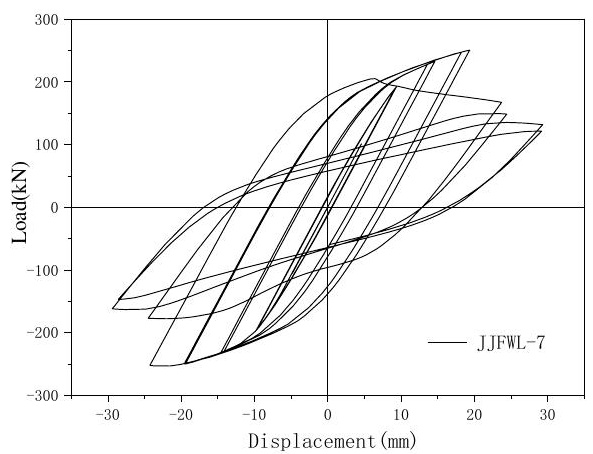

(g) JJFWL-7 phenomenon is different after the peak load. Specimen JJFWL-8 fails owing to the buckling of the hole near the flange, but the other members fail due to the buckling of some pier plates. The hysteresis loops of specimens JJFWL-7 and JJFWL-8 are fuller compared to the other members, so they have a higher energy dissipation capacity.

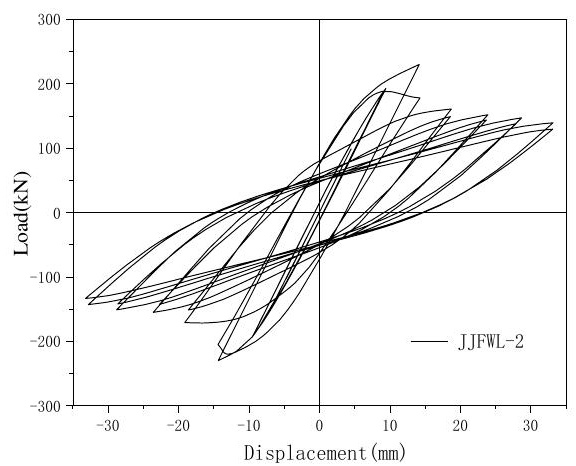

(b) JJFWL-2

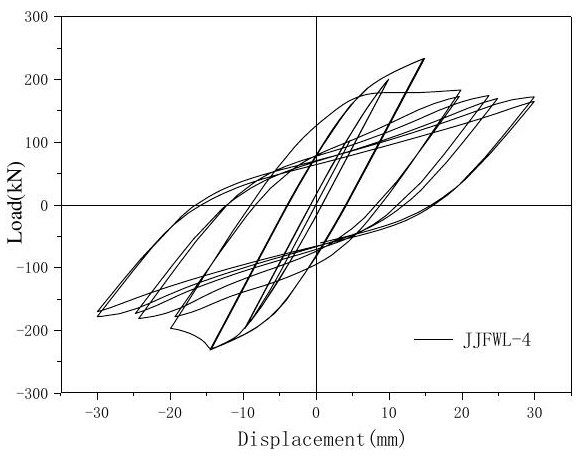

(d) JJFWL-4

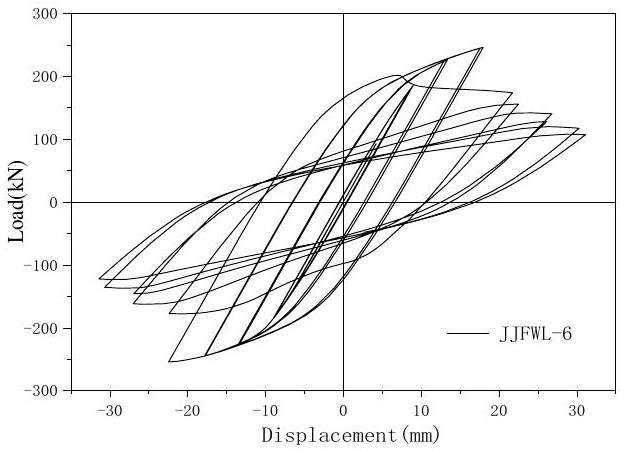

(f) JJFWL-6

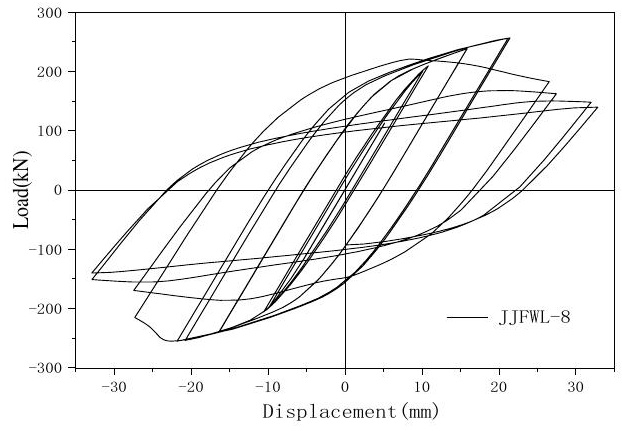

(h) JJFWL-8

Fig. 24 Hysteresis curves of the castellated beams with different arrangements of stiffeners

\subsubsection{Skeleton curves}

Fig. 25 draws the skeleton curves of the members with different arrangements of the stiffening ribs. According to Fig. 25 and Table 7, presenting the relationship between the load and displacement of the specimens, changing the placement of the stiffeners does not vary the initial stiffness of the members but raises their load capacity. The load capacity of specimen JJFWL-1 declines at first, which is attributed to the earlier buckling of the hole pier plate. Specimen JJFWL-8 has the highest load capacity and 
shows a gentler load drop compared to the other components. In all the arrangements of the transverse stiffening ribs, the web of specimen JJFWL-8 does not suffer buckling damage, so its bearing capacity is improved. As reported in Table 7, the peak load of specimens JJFWL-1, JJFWL-2, JJFWL-3, JJFWL-4, JJFWL-5, JJFWL-6, JJFWL-7, and JJFWL-8 is 216.42, 228.19, $233.49,233.53,237.08,251.14,253.15$, and $255.56 \mathrm{kN}$ respectively indicating a corresponding increase of 5.4, 2.3, 0.02, 1.5, 5.9, 0.8, and 0.95\% compared with the previous specimen. The difference in the bearing capacity of specimen JJFWL-1 and that of specimen JJFWL-8 is $39.14 \mathrm{kN}$, denoting an increase of $18.09 \%$, which indicates that the bearing capacity of the castellated members can be greatly improved by setting up transverse stiffeners. Nonetheless, it should be mentioned that the various arrangements of the stiffening ribs have different effects on the bearing capacity of the castellated members.

Table 7

The relation between load and displacement of the specimens

\begin{tabular}{|c|c|c|c|c|c|c|}
\hline \multirow{2}{*}{ Beam ID } & \multicolumn{2}{|c|}{ Yield state } & \multicolumn{2}{|c|}{ Peak state } & \multicolumn{2}{|c|}{ Limit state } \\
\hline & $P_{y}(\mathrm{kN})$ & $\Delta_{y}(\mathrm{~mm})$ & $P_{\max }(\mathrm{kN})$ & $\Delta_{\max }(\mathrm{mm})$ & $P_{u}(\mathrm{kN})$ & $\Delta_{u}(\mathrm{~mm})$ \\
\hline JJFWL-1 & 196.16 & 9.60 & 216.42 & 11.91 & 183.97 & 16.11 \\
\hline JJFWL-2 & 207.11 & 10.12 & 228.19 & 14.29 & 193.99 & 17.15 \\
\hline JJFWL-3 & 208.36 & 10.23 & 233.49 & 14.54 & 198.50 & 17.58 \\
\hline JJFWL-4 & 209.79 & 10.47 & 233.53 & 14.72 & 198.49 & 19.29 \\
\hline JJFWL-5 & 217.20 & 10.74 & 237.08 & 15.61 & 201.52 & 19.88 \\
\hline JJFWL-6 & 218.63 & 11.18 & 251.14 & 22.35 & 213.49 & 22.47 \\
\hline JJFWL-7 & 216.61 & 11.92 & 253.15 & 24.20 & 215.19 & 26.58 \\
\hline JJFWL-8 & 219.88 & 12.18 & 255.56 & 21.93 & 217.23 & 27.90 \\
\hline
\end{tabular}

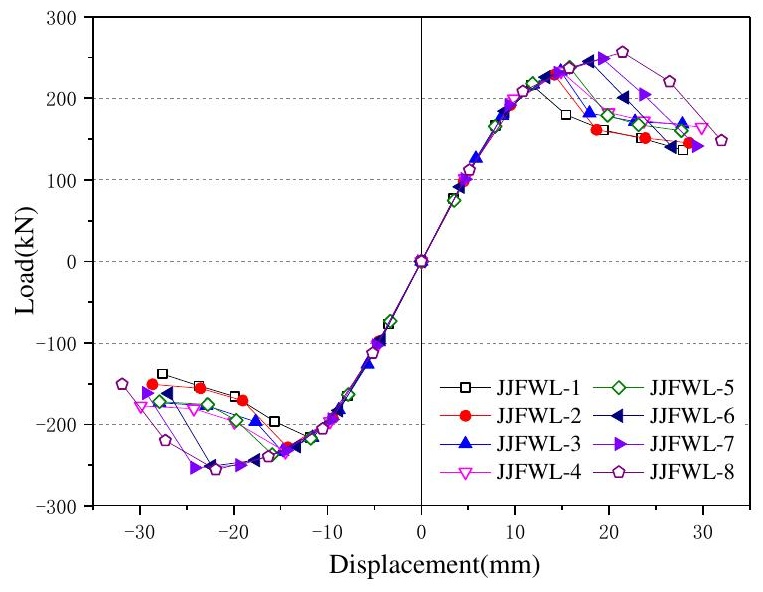

Fig. 25 Skeleton curves of the castellated beams with different arrangements of stiffeners

\subsubsection{Ductility}

The ductility coefficients of the castellated members with different arrangements of the stiffening ribs are listed in Table 8. It is clear that the ductility coefficient of the steel castellated girders is significantly affected by the different arrangements of the stiffeners and increases gradually by changing the position of the stiffeners. Specimens JJFWL-6, JJFWL-7, and JJFWL-8 have a higher ductility coefficient, and specimens JJFWL-7 and JJFWL-8 have a similar ductility coefficient of 2.23 and 2.29 respectively, differing only by $1.35 \%$.

\subsubsection{Energy consumption}

The energy dissipation coefficients and equivalent viscous damping coefficients of the members are presented in Table 9. Varying the placement of the stiffeners gradually raises the energy dissipation capacity of the members. The energy dissipation capacity of specimens JJFWL-7 and JJFWL-8 is 2.072 and 2.411 respectively, which demonstrates that the pier plates near the beam ends do not experience buckling failure, and the energy dissipation capacity of the steel castellated beams can be raised.
Table 8

The ductility coefficients of the specimens

\begin{tabular}{cccc}
\hline Beam ID & $\begin{array}{c}\text { Yield displacement } \\
(\mathrm{mm})\end{array}$ & $\begin{array}{c}\text { Limit displacement } \\
(\mathrm{mm})\end{array}$ & $\begin{array}{c}\text { Ductility } \\
\text { coefficient }\end{array}$ \\
\hline JJFWL-1 & 9.60 & 16.11 & 1.68 \\
JJFWL-2 & 10.12 & 17.15 & 1.69 \\
JJFWL-3 & 10.23 & 17.58 & 1.72 \\
JJFWL-4 & 10.47 & 19.29 & 1.84 \\
JJFWL-5 & 10.74 & 19.88 & 1.85 \\
JJFWL-6 & 11.18 & 22.47 & 2.01 \\
JJFWL-7 & 11.92 & 26.58 & 2.23 \\
JJFWL-8 & 12.18 & 27.90 & 2.29 \\
\hline
\end{tabular}

Table 9

The energy dissipation coefficients of the specimens

\begin{tabular}{ccc}
\hline Beam ID & $\begin{array}{c}\text { Equivalent viscous damping coefficients } \\
\left(h_{e}\right)\end{array}$ & $\begin{array}{c}\text { Energy dissipation } \\
\text { coefficients }(E)\end{array}$ \\
\hline JJFWL-1 & 0.184 & 1.152 \\
JJFWL-2 & 0.188 & 1.185 \\
JJFWL-3 & 0.238 & 1.149 \\
JJFWL-4 & 0.265 & 1.668 \\
JJFWL-5 & 0.267 & 1.678 \\
JJFWL-6 & 0.284 & 1.780 \\
JJFWL-7 & 0.329 & 2.072 \\
JJFWL-8 & 0.384 & 2.411 \\
\hline
\end{tabular}

\subsubsection{Stiffness degradation}

According to Fig. 26, presenting the stiffness degradation curves of the members with different arrangements of the stiffening ribs, the initial stiffness of the steel castellated girders with various arrangements of the stiffeners does not differ considerably. An increase in displacement load causes the stiffness degradation curves of specimens JJFWL-1, JJFWL-2, JJFWL-3, JJFWL-4, and JJFWL-5 to have a steep descending section, which shows that the corresponding arrangements of the transverse stiffeners have a negligible impact on the stiffness of the steel castellated girders. 


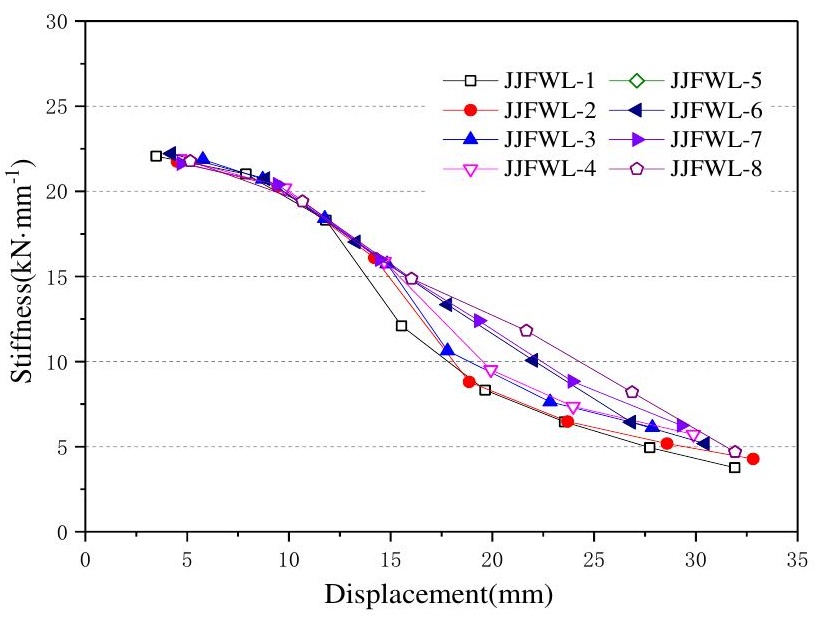

Fig. 26 Stiffness degradation curves of the castellated beams with different arrangements of stiffeners

In summary, through a comprehensive analysis of the different arrangements of the stiffening ribs of the steel castellated beam, we realized that setting up transverse stiffening ribs can greatly improve the hysteretic performance of steel castellated beams. Considering the practical application and engineering economy, a reasonable arrangement of stiffening ribs can effectively enhance the overall performance of the steel castellated girders. The stiffening ribs most obviously affect the first buckling position of the plate.

\section{Conclusions}

To study the hysteretic behavior of steel honeycomb beams with regular hexagonal holes, the impacts of different web height-to-thickness ratios, opening ratios, and arrangements of stiffening ribs on the seismic performance of steel honeycomb beams are studied by means of finite element analysis and experimentation. Based on the results obtained, the following conclusions can

\section{References}

[1] Sweden A.M.I., "Elastic Lateral Stability of I-shaped Cellular Steel Beams", Journal of Constructional Steel Research, 2011, Vol.67, No.2, pp.151-163.

[2] Tsavdaridis K.D. and D'Mello C.,"Web Buckling Study of the Behaviour and Strength of Perforated Steel Beams with Different Novel Web Opening Shapes", Journal of Constructional Steel Research, 2011, Vol.67, No.10, pp.1605-1620.

[3] Feng, R.;Zhan, H.;Meng, S.;Zhu, J."Experiments on H-shaped high-strength steel beams with perforated web",Engineering Structures,2018: 374-394.

[4] Li,X.H., et al."Experimental and Theoretical Study on the Behavior of the Steel-Concrete Composite Beam with Notched Web of Inverted T-shaped Steel Section at Construction Stage",Advanced Steel Construction: An International Journal,2011,Vol.7,No.4,pp.376-386.

[5] Huang B.S. et al., "Calculation method of equivalent bending stiffness of castellated beams and analysis of its influence factors", Journal of Building Structures, 2018, Vol.39, pp.121-127.

[6] Chen D.Y. and Pu W.L., "The Affection of Transverse Stiffener for Elastic Buckling of Castellated Beam Web Under Concentrated Load", International Journal of Science, 2016, Vol.3, No.5.

[7] Anupriya B., Jagadeesa K. and Baskar R., "Experimental investigation of shear strength of castellated beam with and without stiffeners", Journal of Structural Engineering, 2015, Vol.42, No.4, pp. 358-362.

[8] Jia,L.G., Liu,M. and Shi,W.X., "Analysis of the hysteresis performance of the frame node considering the buckling of castellated beam webs", Journal of SJZU University (natural Science Edition), 2016, Vol.6, pp. 961-969. be drawn:

(1) The larger the height-to-thickness ratio of the webs is, the more prone to local buckling the piers are. With the continuous increase of the reciprocating load, the damage accumulates gradually, and the local buckling becomes more and more serious. Moreover, when the components damage, the plastic development of the component is extremely inadequate, and the hysteretic performance is poor.

(2) Reinforcing the steel castellated beams with transverse stiffening ribs and binding the pier slab with the lateral stiffening rib plane effectively prevent the pier plate from buckling and notably improve the hysteretic performance of the beams compared to the non-stiffened steel castellated beams.

(3) Before the buckling of the steel castellated beams, there is little difference in the initial stiffness of the members with or without the transverse stiffening ribs. After buckling deformation, the bearing capacity and deformation ability of the members with the transverse stiffening ribs significantly improve. The larger the height-to-thickness ratio of the member is, the more significant the effect of the stiffening ribs becomes.

(4) The hysteretic performance of the steel castellated girders relates to the arrangement of the stiffening ribs. When the arrangement of the lateral stiffening ribs varies, the failure mode of the steel castellated beams changes. Furthermore, the seismic performance of the steel castellated beams changes when different arrangements of the stiffening ribs are used. However, using a larger number of stiffeners does not necessarily raise the hysteretic performance of the members. The impact of the stiffening ribs is most obvious at the first place where the buckling occurs.

\section{Acknowledgments}

This research work is supported by the National Natural Science Foundation of China (No. 51578346). The tests are conducted in Liaoning Key Lab on Structure and Material of Civil Engineering at Shenyang Jianzhu University. The support provided by the laboratory staff is gratefully acknowledged.

[9] Jia,L.G., et al., "Study on hysteresis performance of castellated beams", Journal of Architectural Science and Engineering, 2018, Vol.35, No.5, pp. 179-187.

[10] Jia L.G. et al., "Shear performance analysis of hexagonal hole castellated beam and castellated composite beam", Engineering Mechanics, 2016, Vol.33, No.1, pp. 81-87.

[11] Showkati H. et al., "Experiments on Elastically Braced Castellated Beams", Journal of Constructional Steel Research, 2012, Vol.77, No.10, pp. 163-172.

[12] Serror M., Hamed A. and Mourad S., "Numerical study on buckling of steel web plates with openings", Steel and Composite Structures, 2016, Vol.22, No.6, pp. 1417-1443.

[13] Pourbehi P. and Pirmoz A., "Shear response of castellated steel beams", International Journal of Steel Structures, 2015, Vol.15, No.2, pp. 389-399.

[14] Ministry of Construction, "Steel and steel products_ Location and preparation of test pieces for mechanical testing", Beijing, China, 1998.

[15] Ministry of Construction, "Metallic Materials-Tensile testing at ambient temperature", Beijing, China, 2010.

[16] Ministry of Construction, "Specification for seismic test of buildings", Beijing, China, 2015.

[17] ABAQUS Standard User's Manual, The Abaqus Software Is a Product of Dassault Systèmes Simulia Corp, Dassault Systèmes, Providence, RI, USA, 2008.

[18] Eiki Yamaguchi."Practical Finite Element Procedure for Achieving Mesh Objectivity in Local Buckling Analysis of Steel Structures by Beam Elements",Advanced Steel Construction: An International Journal,2009,Vol.5,No.3,273-288.

[19] Dong-Ho Choi,Nam-Il Kim."Inelastic Stability Analysis for Framed Structures Subjected to Non-conservative Forces",Advanced Steel Construction: An International Journal,2013,Vol.9,No.4,259-281. 


\title{
AN EFFICIENT METHOD FOR YIELD AND FAILURE SURFACES OF THE STEEL I- SECTION
}

\author{
Anh $\mathrm{Q} V \mathrm{Vu}^{1,{ }^{*}, \text { Nghia H Hoang }}{ }^{2}$, and Hien M Nghiem ${ }^{1}$ \\ ${ }^{I}$ Department of Civil Engineering, Hanoi Architectural University, Hanoi Vietnam \\ ${ }^{2}$ Department of Civil Engineering, Haiphong University, Haiphong Vietnam \\ *(Corresponding author: E-mail: anhvq@hau.edu.vn)
}

\section{A B S T RA C T}

The paper presents a new method to generate efficiently yield and failure surfaces of the doubly symmetrical wide flange I-section under axial force combined with biaxial bending moments in nonlinear analysis of the steel frame. The I-section is divided into several rectangular areas, and the axial force is integrated from uniform stress on each area to form the equilibrium equations of the failure surface. Hyperbolic equations are proposed to approximate the biaxial bending moments of gradually yielding cross-section that represent the yield surfaces lie between limit elastic and failure surfaces. A computer code SPH developed with the implementation of the proposed method aims to perform analyses for numerous cross-sections. The proposed method may help to improve the effectiveness of the finite element analysis of the steel frame in comparison to the fiber method. Several computational examples are conducted to validate the accuracy and efficiency of the proposed method by comparing the results predicted by SPH with those retrieved from other methods.
A R T I C LE H I S T O R Y

$\begin{array}{ll}\text { Received: } & \text { 4 October } 2019 \\ \text { Revised: } & \text { 30 April } 2020 \\ \text { Accepted: } & \text { 30 May } 2020\end{array}$

\section{K E Y W O R D S}

yield surface;

failure surface;

biaxial bending;

steel I-section;

\section{Introduction}

The yield and failure surface concepts had been widely used in inelastic frame analysis to model the full plastification of the steel section under axial force combined with biaxial bending moment (Duan and Chen [1]). Deriving closed-form equations theoretically for the yield and failure surfaces of a general section is complex. For a practical structural analysis, the yield and failure surfaces should be approximated by several simple equations. In the literature, several methods have been presented for the ultimate strength analyses of various sections, such as wide-flange I-section (Orbison et al. [2]), rectangular, circular, and I-sections (Duan and Chen [1]) under axial force combined with biaxial moments. Orbison et al. [2] proposed a polynomial expression for failure surface that contained ratios of axial force and bending moments to the corresponding squash load and plastic moments. Duan and Chen [1] developed smooth and convex failure surfaces in the three-dimension space that satisfied all special cases.

The most promising model for nonlinear analysis of the beam-column element is the fiber method (Taucer et al. [3]). In this method, the cross-section is usually discretized into small fibers, and the nonlinear behavior of the material is represented by the uniaxial stress-strain relationship of a fiber (NgoHuu [4], Ngo-Huu and Kim [5], and Nguyen and Kim [6]). Since the fiber method is the most computationally intensive but more accurate, among others, it is widely applied in theoretical research.

Chiorean [7] proposed a new formulation by which the biaxial interaction diagrams and moment capacity contours of a composite steel-concrete crosssection can be determined, which makes use of an incremental, iterative procedure based on arc-length constraint equations. These methods, based on the fiber approach, may lead to time-consuming in the structural analysis because an iterative solution is usually used in developing the yield and failure surface.

This paper presents an efficient method to generate yield and failure surfaces of the doubly symmetrical wide flange I-section under axial force combined with biaxial bending moments. The proposed method is implemented in a computer program, SPH that developed for nonlinear analysis of the 2D and 3D steel frames. The developed equations for yield and failure surfaces result in a reduction of the time-consuming process in comparison to the fiber method for the finite element analysis of the steel frame.

\section{Section geometry}

Consider the cross-section of I-shape with total height $h$, flange width $b_{f}$, flange thickness $t$, and web thickness $b_{w}$ subjected to the external bending moments about the $\mathrm{Y}$-axis and $\mathrm{Z}$-axis, and axial force in the $\mathrm{X}$-axis as shown in Fig. 1. Assumption can be made as follows: 1) the plane section remains plane after deformation; 2) shear and torsional interaction effects are not considered in the steel constitutive model.

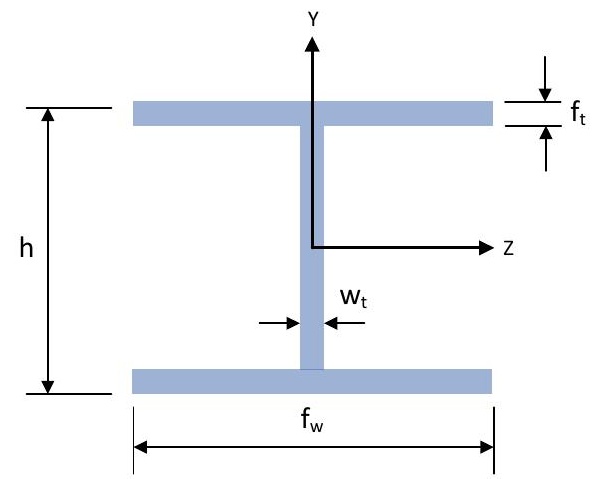

Fig. 1 I-section and dimensions

\section{Steel material}

The stress-strain relationship of steel material, both in tension and in compression, is assumed to be elastic-perfectly plastic, and the strain hardening of steel is neglected, as shown in Fig. 2. The normal stress in the section is calculated as:

$\sigma=E_{S} \varepsilon$

where $E_{s}$ is Young's modulus of steel; $\varepsilon$ is axial strain

The normal stress should not be greater than the yield strength of the steel material, $f_{y}$ :

$-f_{y} \leq \sigma \leq f_{y}$

The yield strength is determined as:

$f_{y}=E_{s} \varepsilon_{y}$

where $\varepsilon_{y}$ is yield axial strain. 


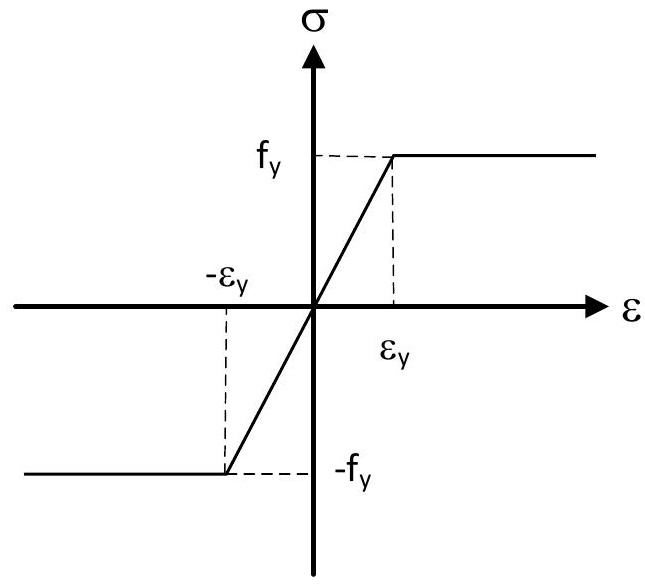

Fig. 2 The stress-strain relationship for steel

\section{Elastic limit surface}

The elastic limit surface separates the space inside the failure surface into two parts, elastic and plastic. This surface is established from a condition that normal stress at furthest location from the neutral axis of a cross-section reaches the yield strength as presented in the following equation:

$\sigma=\frac{P}{A}+\frac{M_{y}}{W_{y}}+\frac{M_{z}}{W_{z}}=f_{y}$

where $A$ is cross-section area; $W_{y}$ and $W_{z}$ are section moduli about $\mathrm{Y}$ and $\mathrm{Z}$ axes, respectively.

The following equation defines the elastic limit surface in terms of nondimensional quantities:

$p+m_{y e 0}+m_{z e 0}=1$

where:

$p=\frac{P}{P_{\max }} ; m_{y e 0}=\frac{M_{y e 0}}{M_{y e}} ; m_{z e 0}=\frac{M_{z e 0}}{M_{z e}}$

where $M_{y e}$ is maximum elastic moment about $\mathrm{Y}$-axis; $M_{z e}$ is maximum elastic moment about Z-axis; $M_{y e 0}$ is moment about $\mathrm{Y}$-axis on the elastic limit surface; $M_{z e 0}$ is moment about $\mathrm{Z}$-axis on the elastic limit surface; $P_{\max }$ is axial strength.

The maximum elastic moments are given by:

$M_{y e}=W_{y} f_{y} ; M_{z e}=W_{z} f_{y}$

The angle between the two components of bending moments is defined as:

$\tan \alpha=\frac{M_{y e 0}}{M_{z e 0}} \frac{I_{z}}{I_{y}}=\frac{m_{y e 0} M_{y e}}{m_{z e 0} M_{z e}} \frac{I_{z}}{I_{y}}$

where $I_{y}$ and $I_{z}$ are moments of inertia about $\mathrm{Y}$ and $\mathrm{Z}$ axes, respectively.

Rearranging Eq. (8) leads to:

$m_{y e 0}=\frac{m_{z e 0} M_{z e}}{M_{y e}} \frac{I_{y}}{I_{z}} \tan \alpha$

Substituting Eq. (9) into Eq. (5):

$m_{z e 0}=\frac{1-p}{M_{y e} I_{z}+M_{z e} I_{y} \tan \alpha} M_{y e} I_{z}$

Substituting Eq. (10) into Eq. (9):

$m_{y e 0}=\frac{1-p}{M_{y e} I_{z}+M_{z e} I_{y} \tan \alpha} M_{z e^{I}} I^{\tan \alpha}$
Finally, the moments on the elastic limit surface are calculated as:

$$
\begin{aligned}
& M_{y e 0}=\frac{1-p}{M_{y e} I_{z}+M_{z e} I_{y} \tan \alpha} M_{z e} I_{y} \tan \alpha M_{y e} \\
& M_{z e 0}=\frac{1-p}{M_{y e} I_{z}+M_{z e} I_{y} \tan \alpha} M_{y e} I_{z} M_{z e}
\end{aligned}
$$

Rearranging Eqs. (12) and (13) leads to simplified equations of the moments on the elastic limit surface:

$$
\begin{gathered}
M_{y e 0}=f_{y} \frac{1-p}{\frac{h}{b_{f}}+\tan \alpha} \tan \alpha W_{y} \\
M_{z e 0}=f_{y} \frac{1-p}{1+\frac{b_{f}}{h} \tan \alpha} W_{z}
\end{gathered}
$$

Since $P_{\max }, M_{y e}$, and $M_{z e}$ are constants for a cross-section, the limit elastic surface is represented by a plane as shown in Fig. 3.

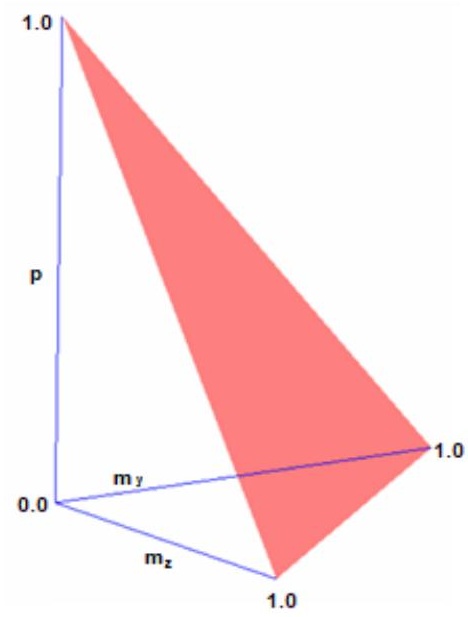

Fig. 3 The limit elastic surface

\section{Failure surface}

At failure, the centroid of the section lies on the neutral axis (NA) because the considered cross-section is doubly symmetrical, as shown in Fig. 4. Stresses over the cross-section reach to yield strength of steel (either compression or tension). The magnitude of the axial force determines the boundary between tension and compression regions. Notations of dimension are used to develop the equations of the failure surface are also shown in Fig. 4 for an I-section under biaxial loading. Based on the distribution of the stresses, the cross-section can be divided into 1) region of axial force which subjects to axial force and 2) regions of bending moment which subject to biaxial bending moment.

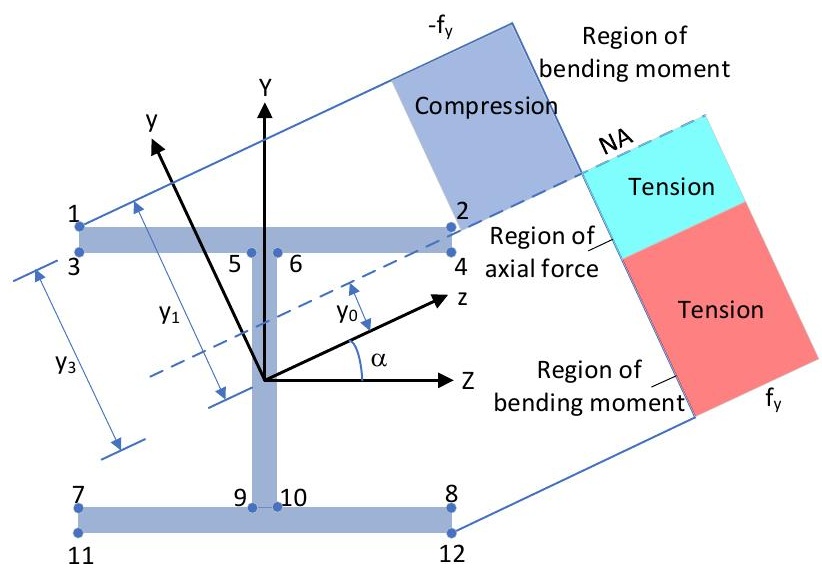

Fig. 4 I-section and dimensions 
A new coordinate system, $y z$ is defined by counterclockwise rotating the $Y Z$ coordinate system an angle of $\alpha$ where the y-axis is parallel to the resultant bending moment. The relationships between coordinates of two coordinate systems for a point on the cross-section are given by:

$z_{i}=Z_{i} \cos \alpha+Y_{i} \sin \alpha$

$y_{i}=-Z_{i} \sin \alpha+Y_{i} \cos \alpha$

where $Y_{i}$ and $Z_{i}$ are coordinates of the $\mathrm{i}^{\text {th }}$ point $(i=1 \div 12)$ in the $Y Z$ coordinate system; $y_{i}$ and $z_{i}$ are coordinates of the $\mathrm{i}^{\text {th }}$ point in the $y z$ coordinate system. The $y_{i}$ coordinate is also the distance from a point on the cross-section to the z-axis. Fig. 4 shows that the distance between the neutral axes before and after yielding is $y_{0}$

With a given axial force, $P$, and angle $\alpha$, the plastic bending moment on the failure surface can be computed by the following procedure:

- Determine axial force $P_{i}$, and the plastic bending moments $M_{y u, i}$, and $M_{z u, i}$ at the $\mathrm{i}^{\text {th }}$ point (Fig. 4) by using the equations shown in the appendix for different cases corresponding to an angle $\alpha$ and $y_{i}>0$ with $i=1 \div 12$, where the neutral axis is passing that point.

- Sort the bending moments and axial forces based on the axial forces from the smallest to the largest where $P_{i}<P_{i+1}, M_{y u, i}<M_{y u, i+1}$, $M_{z u, i}<M_{z u, i+1}$.

- Find $\mathrm{n}$ where $P_{n} \leq P<P_{n+1}$.

- Interpolate the distance from the center of the cross-section to the neutral axis, corresponding to the axial force, $P$, using the following equation:

$$
y=y_{n}+\frac{P-P_{n}}{P_{n+1}-P_{n}}\left(y_{n+1}-y_{n}\right)
$$

- Determine the plastic bending moment $M_{y u}, M_{z u}$ and axial force $\bar{P}$ from a distance $y$ calculated in Eq. (17) by using the equations in the appendix. If calculated axial force $\bar{P}$ is different from the axial force $P$, an iterative computation needs to conduct to find the distance $y$ which satisfied an error control $|(\bar{P}-P) / P| \leq 1 E-3$.

The failure surface is plotted in Fig. 5.

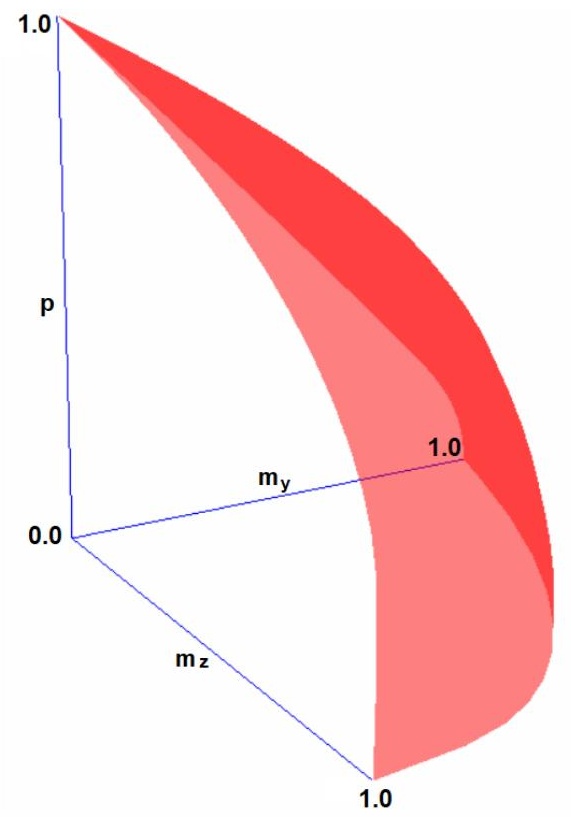

Fig. 5 Failure and yield surfaces

Examples of the failure surfaces are performed for the wide-flange Isections W12x30 (light-weight) and W14x426 (heavy-weight), respectively. The proposed method considered as an exact solution compared to the fiber method and Duan and Chen [1], as shown in Figs. 6 and 7. Fig. 6a shows the comparison of three solutions for the light-weight section W12x30 for the cases of $\mathrm{p}=0,0.5$, and 0.8 , and Fig. 7a is for the heavy-weight section $\mathrm{W} 14 \mathrm{x} 426$. Referring to those figures, the results of the proposed method are matched very well with the fiber method, while the results of Duan and Chen' method [1] are relatively agreed. The failure surfaces under the strong axis (Z-axis) obtained from the proposed method compared to the fiber and the Duan and Chen' method [1] in Figs. $6 \mathrm{~b}$ and $7 \mathrm{~b}$. All three methods produced very good agreement results except for the region of lower axial force where the Duan and Chen' method [1] is slightly less than those from the other methods. For the weak-axis (Y-axis) bending of wide-flange sections, it can be seen that the proposed method and two others' results are very close for both sections W12x30 and W14x426, as shown in Fig. 6d and 7d.

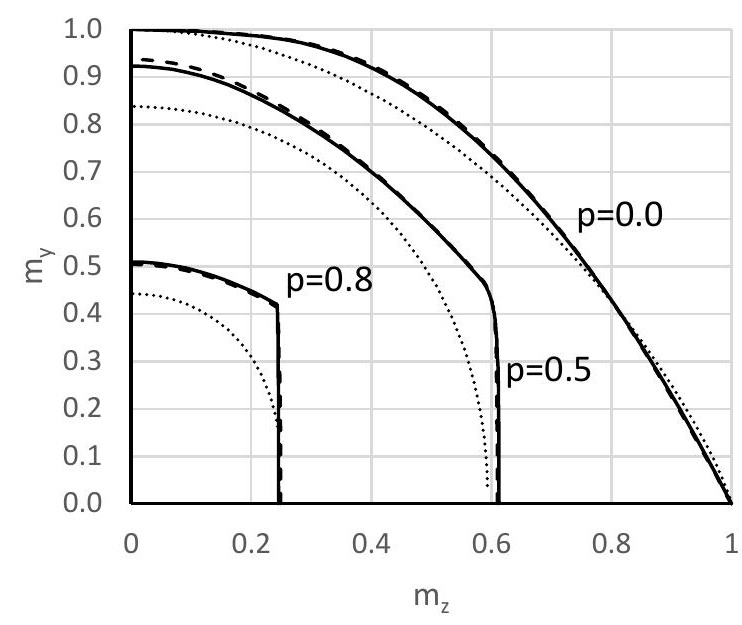

a) Plane view of the failure surfaces

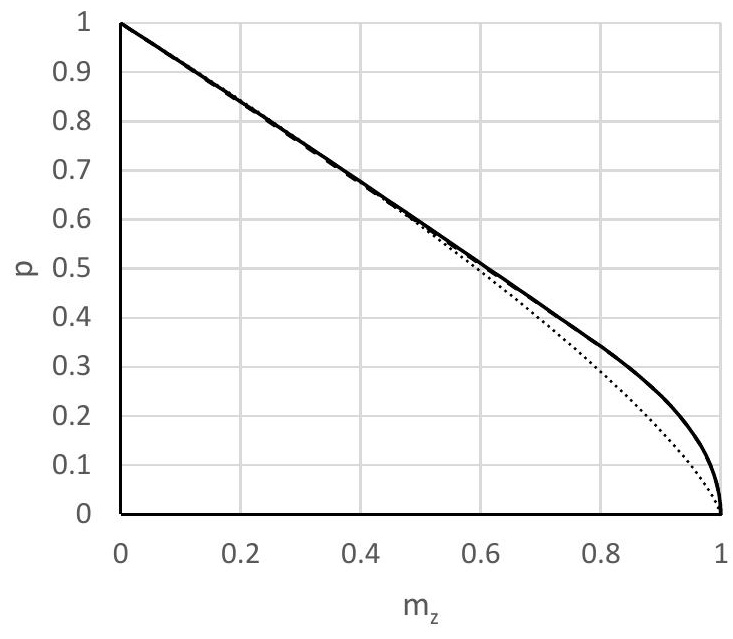

b) Failure surfaces under strong axis (Z-axis)

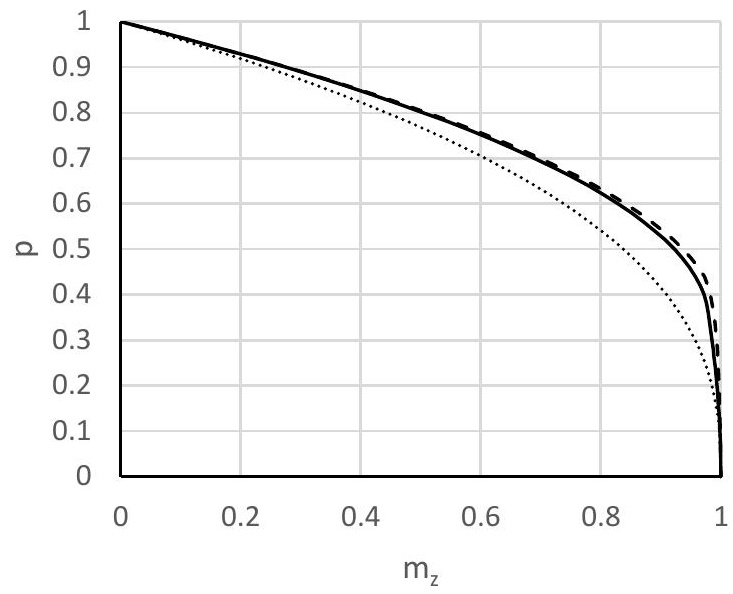

- - - - The fiber method

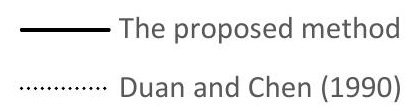

c) Failure surfaces under weak axis (Y-axis)

Fig. 6 Comparison of the failure surface for W12x30 


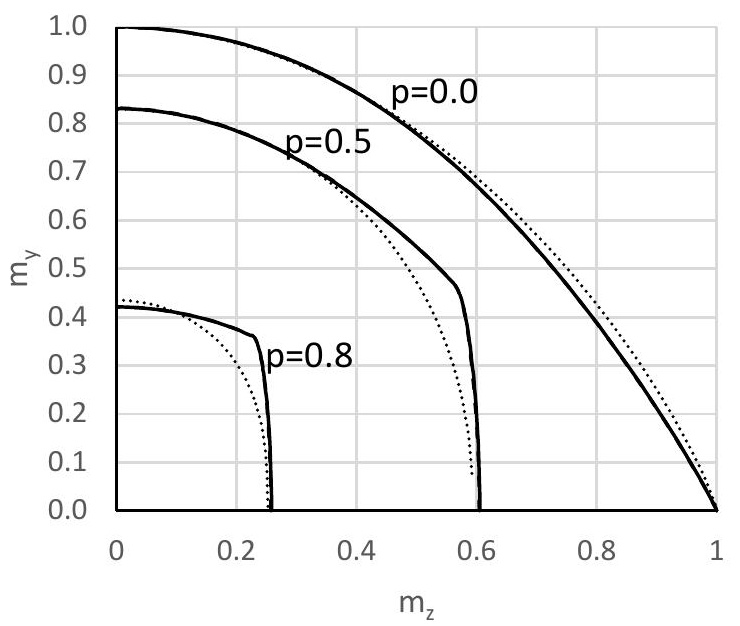

a) Plane view of the failure surfaces

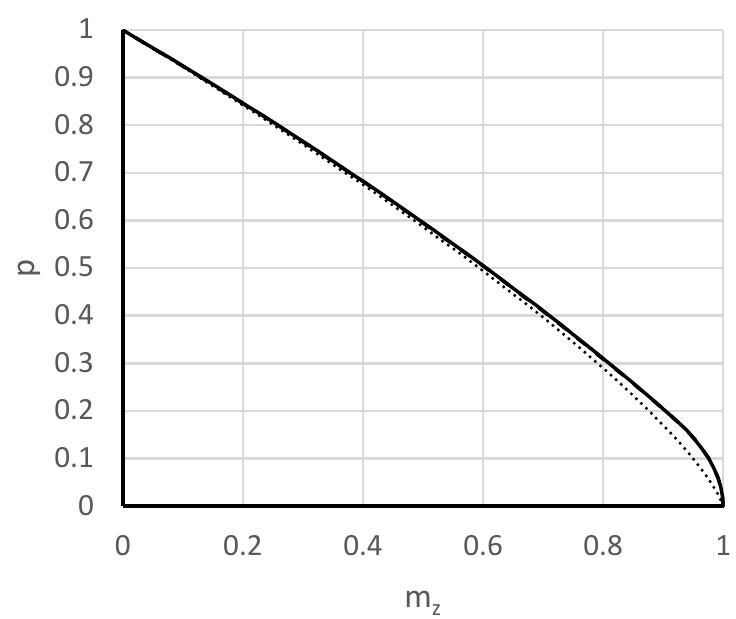

b) Failure surfaces under strong axis (Z-axis)

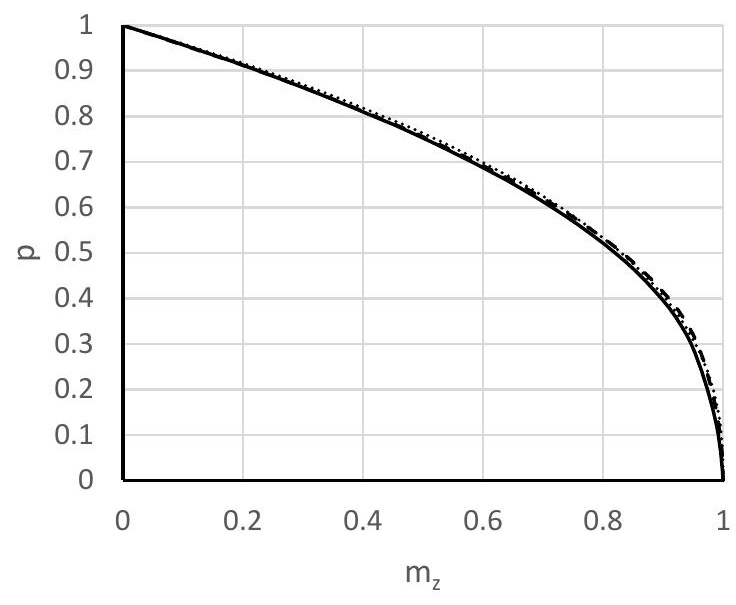

- - - - The fiber method

The proposed method

Duan and Chen (1990)

c) Failure surfaces under weak axis (Y-axis)

Fig. 7 Comparison of the failure surface for W14x426

\section{Yield surface}

The yield surfaces located within the elastic limit surface and the failure surface represent the gradual yielding of the cross-section. The relationship between bending moment and the unit rotation angle is nonlinear and can be approximated by hyperbolic curves, as shown in Fig. 8 and presented in the following equations:
If $M_{y u}>M_{y e 0}:$

$$
M_{y}=M_{y e 0}+\frac{\phi_{y}-\phi_{y e 0}}{\frac{1}{E I_{y}}+\frac{\phi_{y}-\phi_{y e 0}}{M_{y u}-M_{y e 0}}}
$$

If $M_{z u}>M_{z e 0}:$

$$
M_{z}=M_{z e 0}+\frac{\phi_{z}-\phi_{z e 0}}{\frac{1}{E I_{z}}+\frac{\phi_{z}-\phi_{z e 0}}{M_{z u}-M_{z e 0}}}
$$

If $M_{y u}<M_{y e 0}$ :

$$
M_{y}=M_{y e 0}-\frac{\phi_{y}-\phi_{y e 0}}{\frac{1}{E I_{y}}+\frac{\phi_{y}-\phi_{y e 0}}{M_{y e 0}-M_{y u}}}
$$

If $M_{z u}<M_{z e 0}:$

$$
M_{z}=M_{z e 0}-\frac{\phi_{z}-\phi_{z e 0}}{\frac{1}{E I_{z}}+\frac{\phi_{z}-\phi_{z e 0}}{M_{z e 0}-M_{z u}}}
$$

where $M_{y u}$ and $M_{z u}$ are plastic bending moments about y and $\mathrm{z}$ axes, respectively; $\phi_{y}$ and $\phi_{z}$ are unit rotation angles in $\mathrm{y}$ and $\mathrm{z}$ directions, respectively; and $\phi_{y e 0}$ and $\phi_{z e 0}$ are unit rotation angles at elastic limit of the section in $\mathrm{y}$ and $\mathrm{z}$ directions, respectively.

The curves for the moment-unit rotation angle relationship contain three segments: 1) straight line for the elastic state; 2) hyperbolic curve for yielding state; and 3) horizontal straight line for failure state.

The wide flange sections $\mathrm{W} 12 \times 30$ and $\mathrm{W} 14 \times 426$ are selected again to analyze and compare to the fiber method. The moment-unit rotation angle curves for the W12x30 section are plotted in Fig. 9 and Fig. 10. The curves from the proposed method match quite well with those from the fiber except the cases of $\mathrm{p}=0.5, \alpha=90^{0}$, and $\alpha=75^{\circ}$ where the proposed method produced slightly lower bending moment. Fig. 12 shows the moment-unit rotation angle curves for W14x426 section. Good agreements are also obtained from comparing those curves with the fiber method.

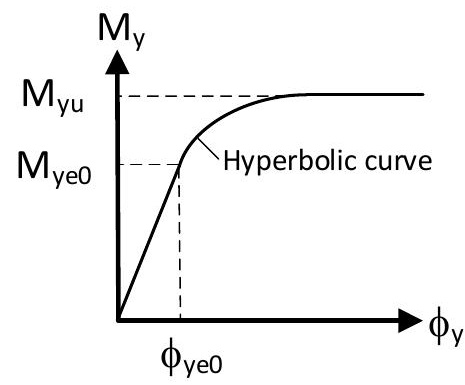

(a) $\mathrm{M}_{\mathrm{y}}-\phi_{\mathrm{y}}$;

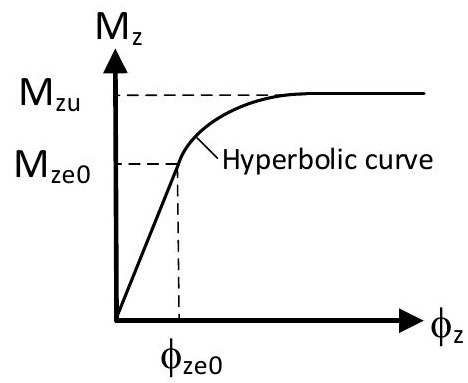

(b) $M_{z}-\phi_{z}$

Fig. 8 Moment-unit rotation angle relationship 


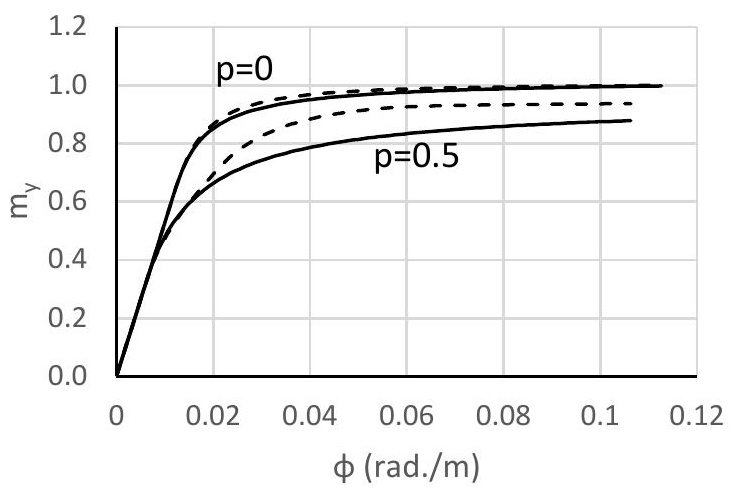

(a) $\alpha=90^{\circ}$

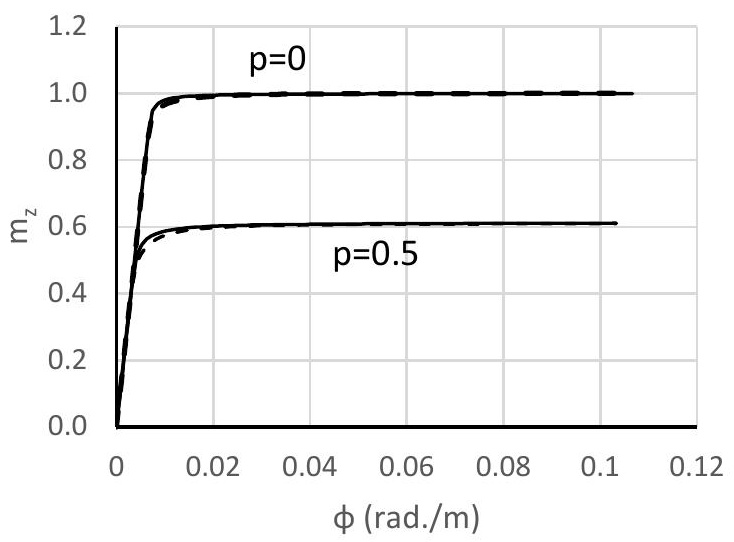

The proposed method - - - The fiber method

(b) $\alpha=0^{0}$

Fig. 9 Moment-unit rotation angle relationships for $\mathrm{W} 12 \times 30$ at $\alpha=90^{\circ}$ and $\alpha=0^{0}$
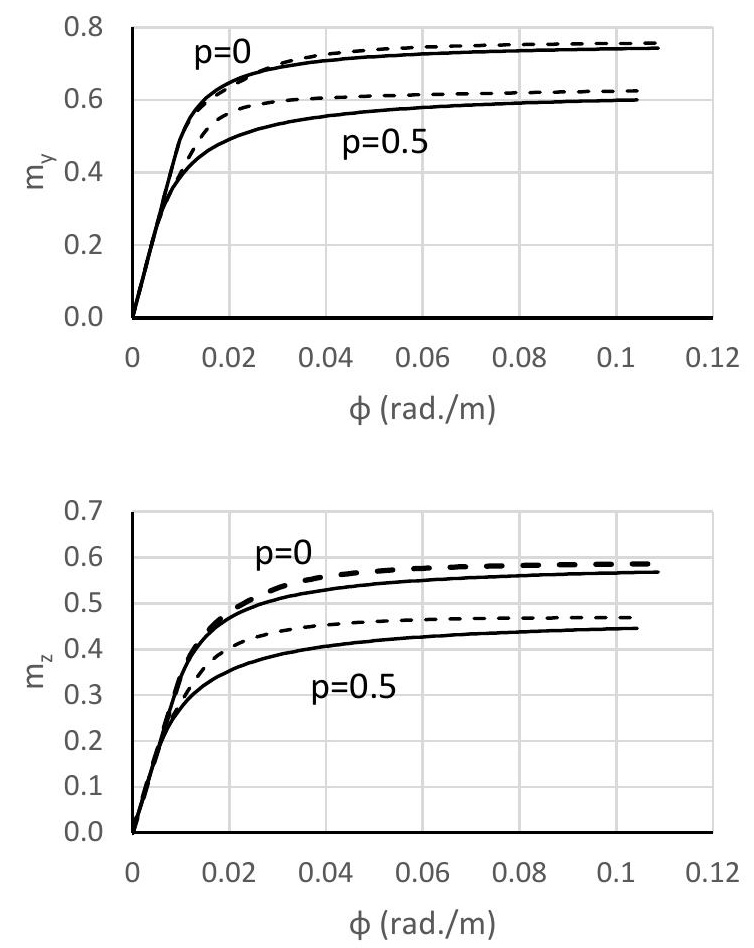

— The proposed method - - - The fiber method

Fig. 10 Moment-unit rotation angle relationships $\alpha=75^{\circ}$ for $\mathrm{W} 12 \times 30$

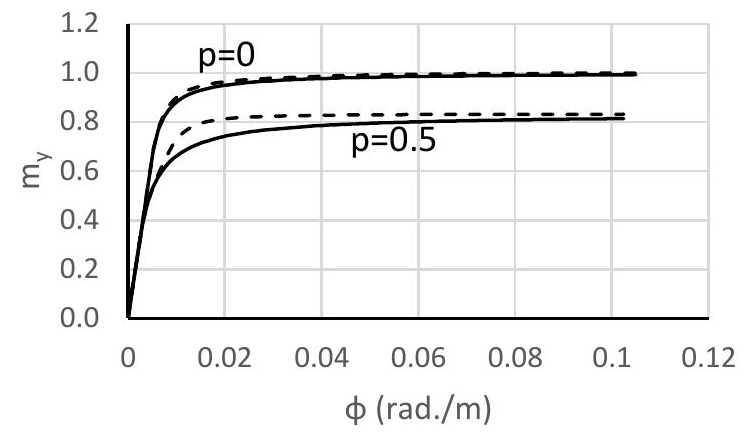

(a) $\alpha=90^{0}$

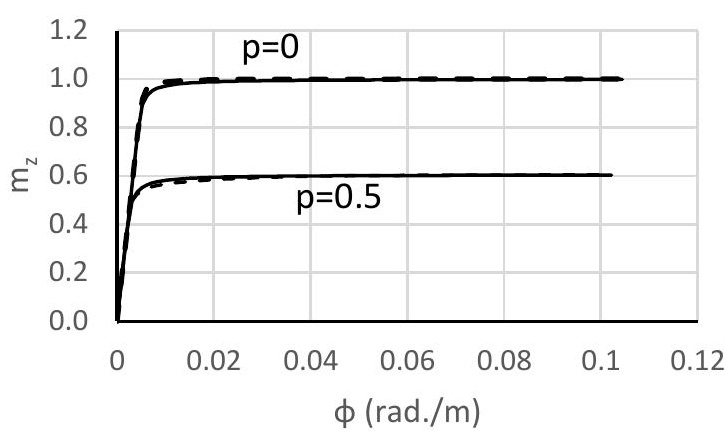

— The proposed method - - - The fiber method

(b) $\alpha=0^{0}$

Fig. 11 Moment-unit rotation angle relationships for W14x426 at $\alpha=90^{\circ}$ and $\alpha=0^{0}$
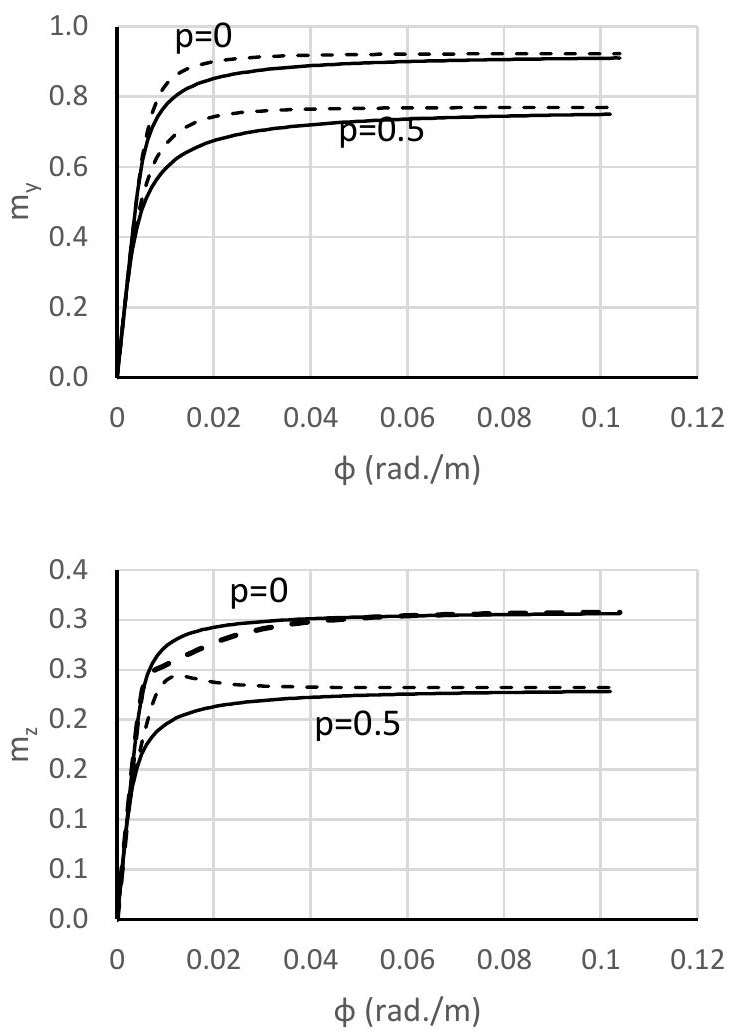

— The proposed method - - - The fiber method

Fig. 12 Moment-unit rotation angle relationships $\alpha=75^{\circ}$ for $\mathrm{W} 12 \times 30$

\section{Numerical analyses and results}

The following analyses for steel structures are conducted to verify the accuracy of the proposed method. The SPH program also can be used to predict the nonlinear behavior of plane and space frames. The proposed method is compared well to other solutions. 
A steel portal frame shown in Fig. 13 consists of a wide flange steel beam of W12x17 and two columns of W12x50. This frame has been analyzed by Liew et al. [8], Iu et al. [9], and Chiorean [10]. Plastic hinges are allowed to occur at only two ends of each element. In this study, the behavior of a steel frame is investigated. The geometry, section properties, and loading arrangement of the steel portal frame are depicted in Fig. 13. The frame is subjected to both vertical and lateral loading applied proportionally. The yield strength of all elements is 248.2 $\mathrm{MPa}$, and Young's modulus is E=20000 MPa. The nonlinear behaviors represented by the load-displacements curves at the point A computed by the proposed method and those given in Liew et al. [8], Iu et al. [9] and Chiorean [10] are compared in Fig. 14. It can be observed that the results agree very well.

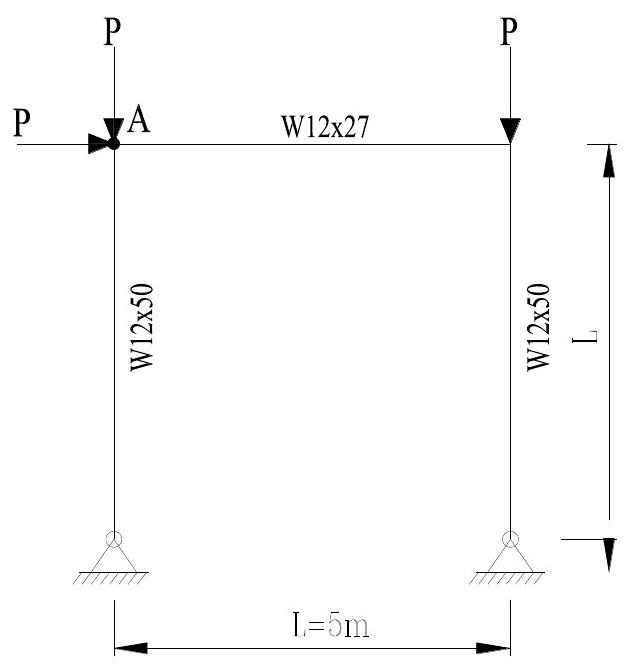

Fig. 13 Steel portal frame

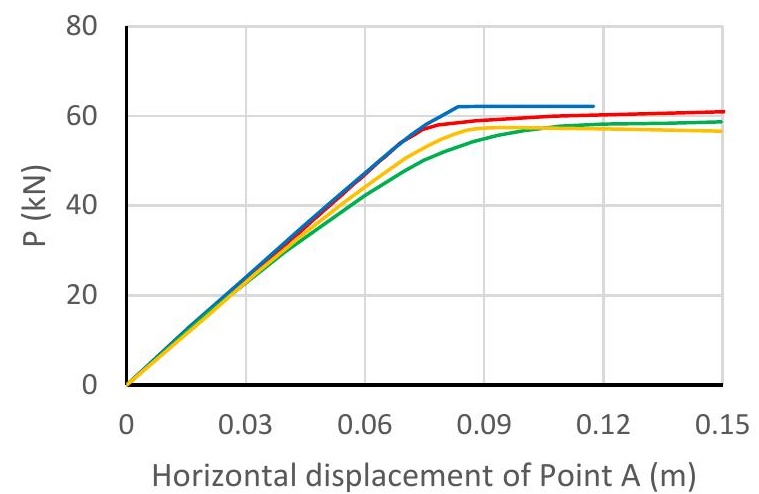

The proposed method

- Liew et al. (2001)

lu et al (2009)

Chiorean (2013)

Fig. 14 Load-displacement curve for point A of the steel portal frame

Fig. 15 shows Orbison's six-story space frame. This frame was analyzed by several researchers such as Liew et al. [11], Kim et al. [12,13], Kim and Choi [14], and Skordeli and Bisbos [15]. The yield strength of all members is 250 $\mathrm{MPa}$ (36 ksi) and Young's modulus is $206850 \mathrm{MPa}$ (30000 ksi). Uniform floor pressure of $4.8 \mathrm{KN} / \mathrm{m} 2$ ( $100 \mathrm{psf}$ ) is converted into equivalent concentrated loads on the top of the columns. Wind loads are simulated by point loads of $26.7 \mathrm{KN}$ (6 kips) in the $Y$-direction at every beam-column joints and only applied on the front elevation. The load-displacement curve at point $\mathrm{A}$ in the $\mathrm{Y}$-direction predicted by the proposed analysis compared well with that of Liew et al. [11], as shown in Fig. 16. The ultimate load factor calculated from the proposed method is 2.1 , that nearly equivalent to 2.062 calculated by Liew et al. [11].

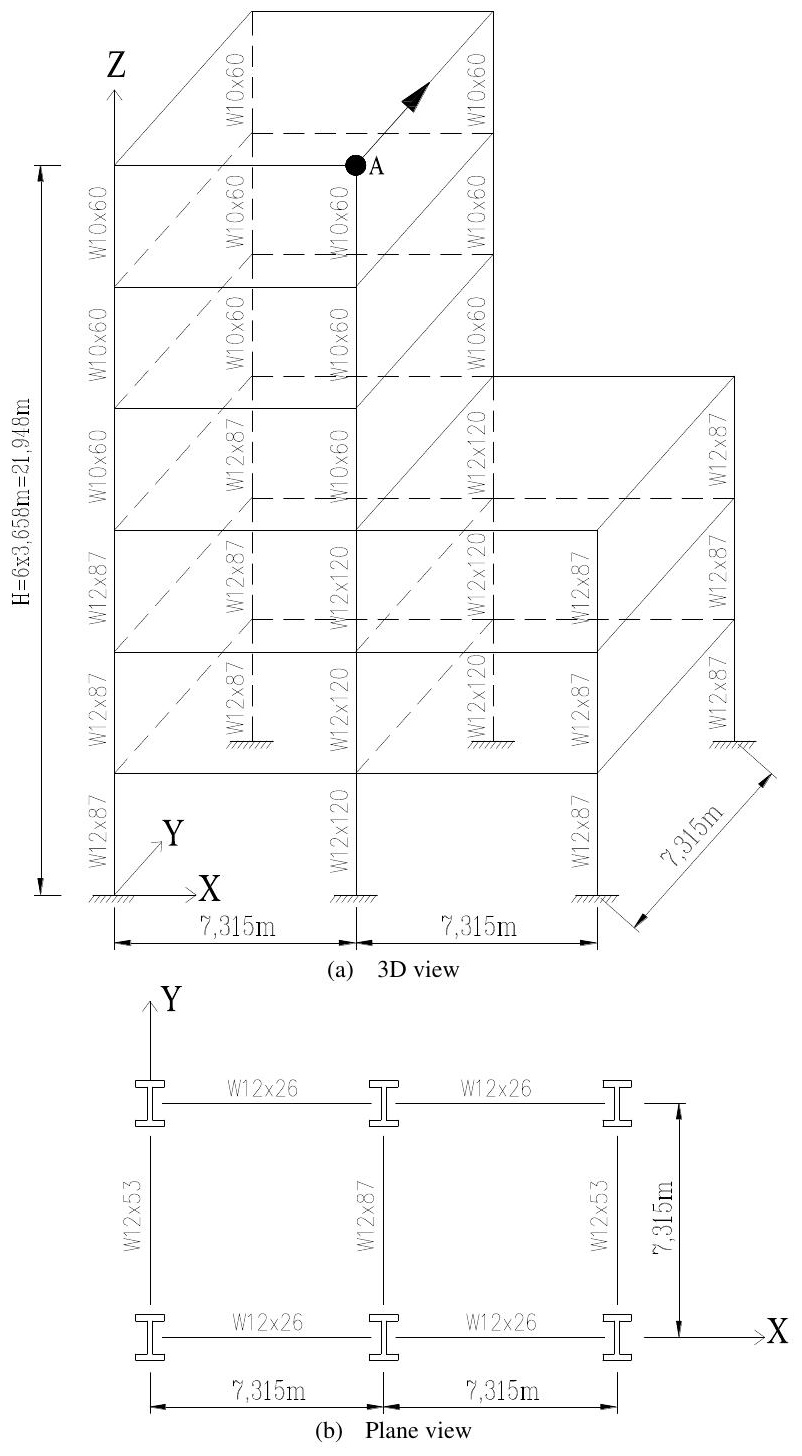

Fig. 15 Six-story space frame

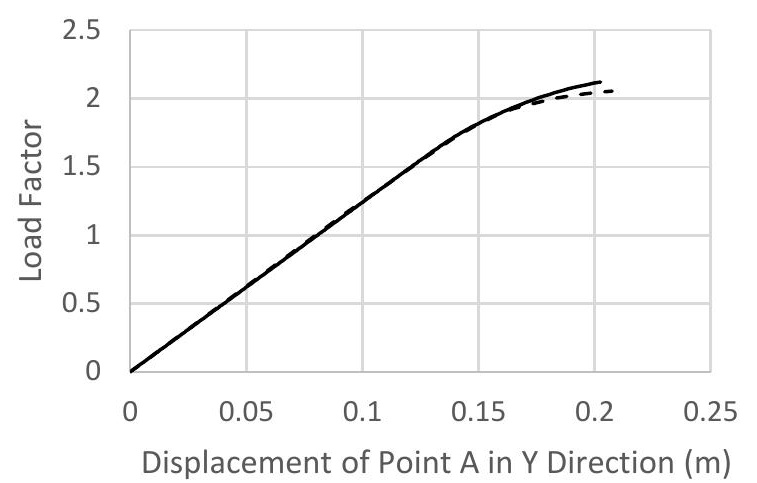

The proposed method - - - - Liew et al. (1990)

Fig. 16 The load-displacement curve at point $\mathrm{A}$ in the $\mathrm{Y}$ direction

\section{Conclusion}

A new method to develop the yield and failure surfaces for commonly used I-section in steel structure under biaxial loading is proposed. The equations of yield and failure surfaces are implemented in the nonlinear static analysis program SPH. Comparisons the predicted moment-unit rotation angle curves and failure surfaces with those from the fiber method confirm the validity of the proposed method. Load-displacement curves produced from the nonlinear analyses of the 2D and 3D steel frames using the proposed method agreed well with those from the available methods in the literature. The developed equations for yield and failure surfaces can help to save computational time in comparison to the fiber method. 


\section{Appendix}

- Case 1

If $y_{0} \geq y_{4} ; y_{0}<y_{2} ; y_{0}<y_{3}$ (Fig. A1)
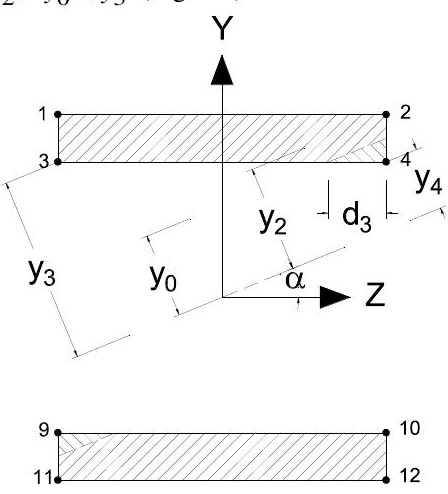

Fig. A1

$d_{3}=\left(y_{0}-y_{4}\right) / \sin \alpha$

$P=f_{y} d_{3}^{2} \tan \alpha$

$M_{Y}=f_{y} d_{3}^{2} \tan \alpha\left(\frac{b_{f}}{2}-\frac{d_{3}}{3}\right)$

$M_{Z}=f_{y}\left(b_{f} t(h-t)-d_{3}^{2} \tan \alpha\left(\frac{h}{2}-t+\frac{d_{3} \tan \alpha}{3}\right)\right)$

- $\quad$ Case 2

If $y_{2} \leq y_{0}<y_{3}$ (Fig. A2)
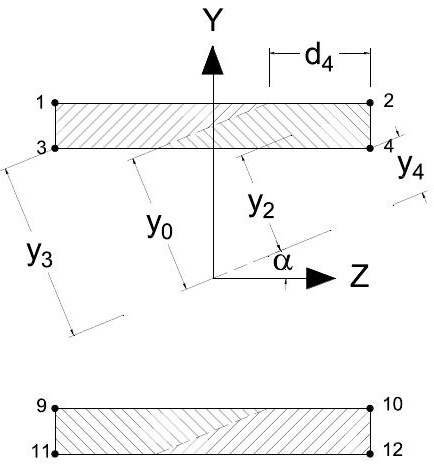

Fig. A2

$d_{4}=\left(y_{0}-y_{2}\right) / \sin \alpha$

$P=f_{y} t\left(\frac{t}{\tan \alpha}+2 d_{4}\right)$

$M_{Y}=f_{y} t\left(\left(b_{f}-d_{4}-\frac{t}{\tan \alpha}\right)\left(d_{4}+\frac{t}{\tan \alpha}\right)\right)$

$+f_{y} \frac{t^{2}}{\tan \alpha}\left(d_{4}+\frac{2 t}{3 \tan \alpha}-\frac{b_{f}}{2}\right)$

$M_{Z}=f_{y}\left(t\left(b_{f}-d_{4}-\frac{t}{\tan \alpha}\right)(h-t)+\frac{t^{2}}{\tan \alpha}\left(\frac{h}{2}-\frac{t}{3}\right)\right)$

\section{- $\quad$ Case 3}

If $y_{3} \leq y_{0}<y_{1}$ (Fig. A3)
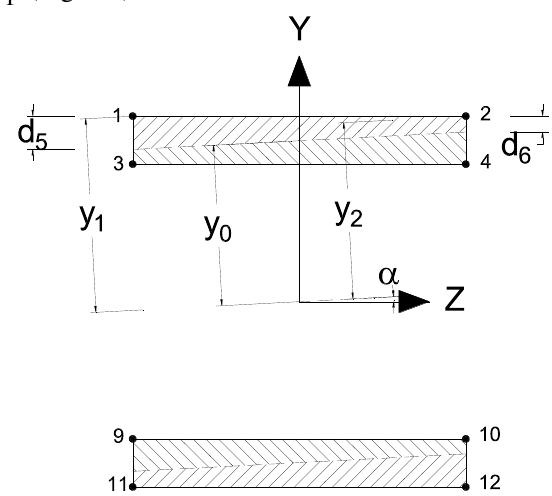

Fig. A3 $d_{5}=\left(y_{1}-y_{0}\right) / \cos \alpha ; d_{6}=\left(y_{2}-y_{0}\right) / \cos \alpha$

$P=2 f_{y} b_{f}\left(t-\frac{d_{5}+d_{6}}{2}\right)$

$M_{Y}=f_{y}\left(d_{5}-d_{6}\right) \frac{b_{f}^{2}}{6}$

$M_{Z}=f_{y}\left(d_{6} b_{f}\left(h-d_{6}\right)+\left(d_{5}-d_{6}\right) b_{f}\left(\frac{h}{2}-d_{6}-\frac{d_{5}-d_{6}}{3}\right)\right)$

- $\quad$ Case 4

If $y_{3} \leq y_{0}<y_{2}$ (Fig. A4)

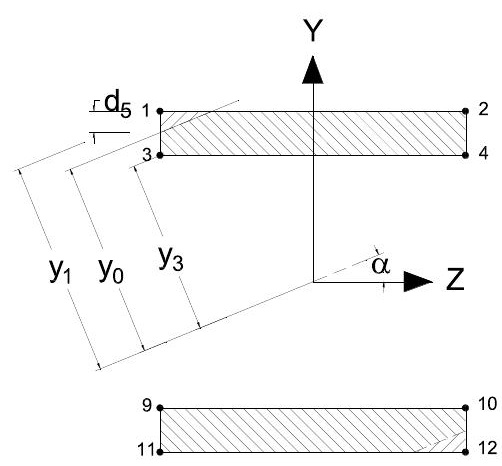

Fig. A4

$d_{5}=\left(y_{1}-y_{0}\right) / \cos \alpha$

$P=2 f_{y}\left(b_{f} t-\frac{1}{2} \frac{d_{5}^{2}}{\tan \alpha}\right)$

$M_{Y}=f_{y} \frac{d_{5}^{2}}{\tan \alpha}\left(\frac{b_{f}}{2}-\frac{d_{5}}{3 \tan \alpha}\right)$

$M_{Z}=f_{y} \frac{d_{5}^{2}}{\tan \alpha}\left(\frac{h}{2}-\frac{d_{5}}{3}\right)$

- $\quad$ Case 5

If $y_{2} \leq y_{0}<y_{3}$ and $\frac{h-2 t}{b_{f}} \leq \tan \alpha<\frac{h}{b_{f}}$ (Fig. A5)
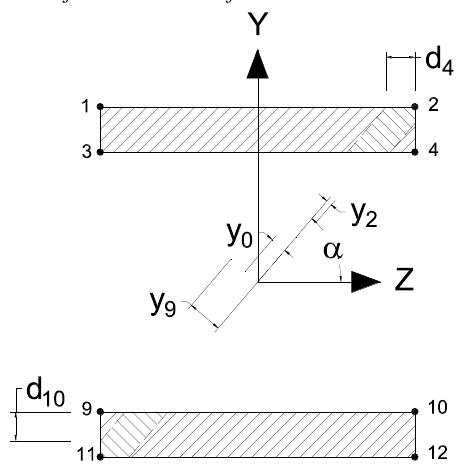

Fig. A5

$d_{4}=\left(y_{0}-y_{2}\right) / \sin \alpha ; d_{10}=\left(y_{9}-y_{0}\right) / \cos \alpha$

$$
\begin{aligned}
P= & f_{y} t\left(\frac{t}{\tan \alpha}+2 d_{4}\right)-f_{y} \frac{d_{10}^{2}}{\tan \alpha} \\
M_{Y}= & 2 f_{y} t\left(b_{f}-d_{4}-\frac{t}{\tan \alpha}\right)\left(\frac{d_{4}}{2}+\frac{t}{2 \tan \alpha}\right) \\
& +f_{y} \frac{t^{2}}{\tan \alpha}\left(d_{4}+\frac{2 t}{3 \tan \alpha}-\frac{b_{f}}{2}\right) \\
& +f_{y} \frac{d_{10}^{2}}{\tan \alpha}\left(\frac{b_{f}}{2}-\frac{d_{10}}{3 \tan \alpha}\right) \\
M_{Z}= & f_{y}\left(t\left(b_{f}-d_{4}-\frac{t}{\tan \alpha}\right)(h-t)+\frac{t^{2}}{\tan \alpha}\left(\frac{h}{2}-\frac{t}{3}\right)\right) \\
& -f_{y} \frac{d_{10}^{2}}{\tan \alpha}\left(\frac{h}{2}-t+\frac{d_{10}}{3}\right)
\end{aligned}
$$




\section{- Case 6}

If $y_{2} \leq y_{0}<y_{3}$ and $\frac{h}{b_{f}} \leq \tan \alpha$ (Fig. A6)

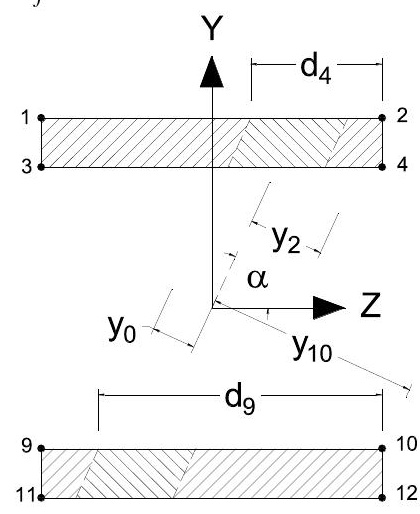

Fig. A6

$d_{4}=\left(y_{0}-y_{2}\right) / \sin \alpha ; d_{9}=\left(y_{0}-y_{10}\right) / \sin \alpha$

$$
\begin{aligned}
P= & f_{y} t\left(\frac{t}{\tan \alpha}+2 d_{4}\right)-f_{y} t\left(2 b_{f}-\frac{t}{\tan \alpha}-2 d_{9}\right) \\
M_{Y} & =f_{y} t\left(\left(b_{f}-d_{4}-\frac{t}{\tan \alpha}\right)\left(d_{4}+\frac{t}{\tan \alpha}\right)\right) \\
& +f_{y} \frac{t^{2}}{\tan \alpha}\left(d_{4}+\frac{2 t}{3 \tan \alpha}-\frac{b_{f}}{2}\right) \\
& +f_{y} t\left(\left(b_{f}-d_{9}-\frac{t}{\tan \alpha}\right)\left(d_{9}+\frac{t}{\tan \alpha}\right)\right) \\
& +f_{y} \frac{t^{2}}{\tan \alpha}\left(d_{9}+\frac{2 t}{3 \tan \alpha}-\frac{b_{f}}{2}\right) \\
M_{Z} & =f_{y} t(h-t)\left(d_{9}-d_{4}\right)
\end{aligned}
$$

\section{- $\quad$ Case 7}

If $y_{0} \leq y_{6}$ and $y_{0} \geq y_{7}$ (Fig. A7)

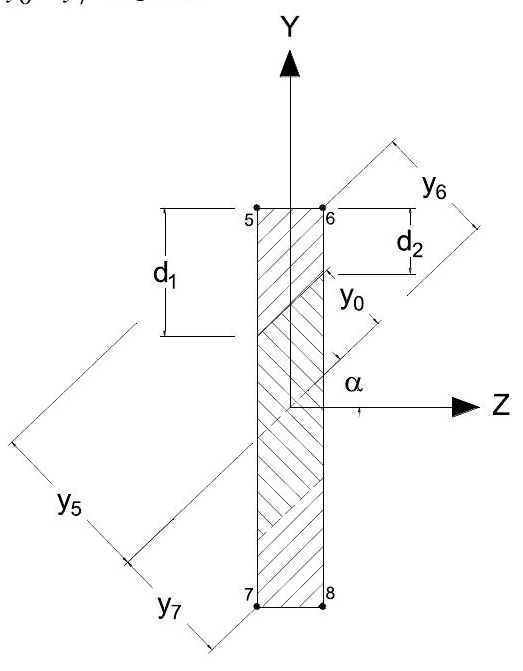

Fig. A7

$d_{1}=\left(y_{5}-y_{0}\right) / \cos \alpha ; d_{2}=\left(y_{6}-y_{0}\right) / \cos \alpha$

$P=f_{y} b_{w}\left(h-2 t-\left(d_{1}+d_{2}\right)\right)$

$M_{Y}=\frac{1}{6} f_{y} b_{w}^{3} \tan \alpha$

$M_{Z}=f_{y} d_{2} b_{w}\left(h-2 t-d_{2}\right)$

$$
+f_{y} b_{w}^{2} \tan \alpha\left(\frac{h}{2}-t-d_{2}-\frac{b_{w} \tan \alpha}{3}\right)
$$

\section{- $\quad$ Case 8}

If $y_{0} \leq y_{5}, y_{0}>y_{6}$, and $y_{0}<y_{7}$ (Fig. A8)

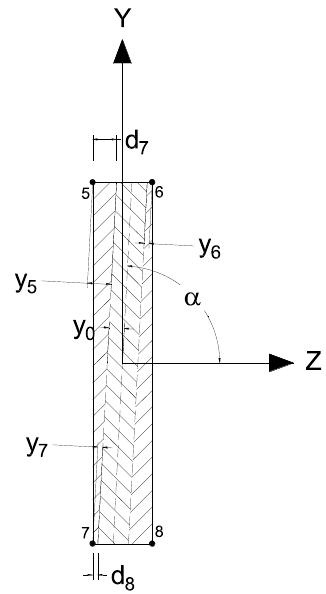

Fig. $A 8$

$d_{7}=\left(y_{5}-y_{0}\right) / \sin \alpha ; d_{8}=\left(y_{7}-y_{0}\right) / \sin \alpha$

$P=f_{y}\left(b_{w}-d_{7}-d_{8}\right)(h-2 t)$

$M_{Y}=f_{y} d_{8}\left(b_{w}-d_{8}\right)(h-2 t)$

$+f_{y}\left(d_{7}-d_{8}\right)(h-2 t)\left(\frac{b_{w}}{2}-\frac{d_{7}+2 d_{8}}{3}\right)$

$M_{Z}=\frac{1}{6} f_{y}\left(\left(d_{7}-d_{8}\right)(h-2 t)^{2}\right)$

- $\quad$ Case 9

If $y_{0} \leq y_{5}, y_{0}>y_{6}$, and $y_{0}>y_{7}$ (Fig. A9)

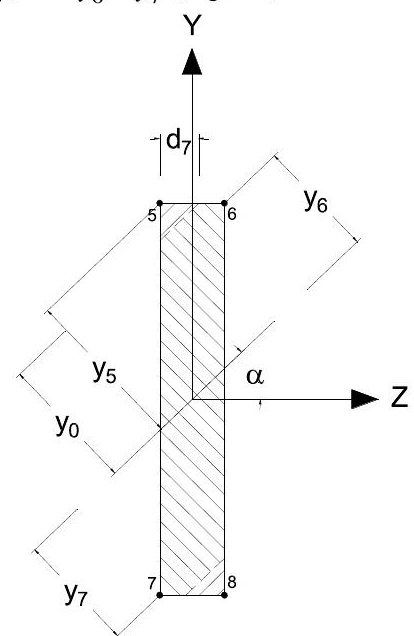

Fig. A9

$$
\begin{aligned}
& d_{7}=\left(y_{5}-y_{0}\right) / \sin \alpha \\
& P=f_{y}\left(b_{w}(h-2 t)-d_{7}^{2} \tan \alpha\right) \\
& M_{Y}=f_{y} d_{7}^{2} \tan \alpha\left(\frac{b_{w}}{2}-\frac{d_{7}}{3}\right) \\
& M_{Z}=f_{y} d_{7}^{2} \tan \alpha\left(\frac{h}{2}-t-\frac{d_{7} \tan \alpha}{3}\right)
\end{aligned}
$$

\section{References}

[1] Duan, L., and Chen, W.F., (1990), "A yield surface equation for doubly symmetrical sections", Eng. Struc., 12; 114-119. https://doi.org/10.1016/0141-0296(90)90016-L

[2] Orbison, J. G., McGuire, M., and Abel, J.F. (1982), "Yield surface application in nonlinear steel frame analysis", Computer methods in applied mechanics and engineering, 33; 557-573. https://doi.org/10.1016/0045-7825(82)90122-0

[3] Taucer, F., Spacone, E., and Filippou, F.C. (1991). A Fiber Beam-Column Element for Seismic Response Analysis of Reinforced Concrete Structures. Berkekey, California: Earthquake Engineering Research Center, College of Engineering, University of California, 91(17)

[4] Ngo Huu, C. (2006), "Practical advanced analysis of steel-concrete composite structures using fiber-hinge method", Ph.D. dissertation, Department of Civil and Environmental Engineering, the Graduate school of Sejong University.

[5] Ngo, H.C., and Kim, S.E. (2009), "Practical advanced analysis of space steel frames using fiber hinge method", Thin-Walled Structures, 47(4), 421-430.

https://doi.org/10.1016/j.tws.2008.08.007 
[6] Nguyen, P.C., and Kim, S.E., (2015), "Second-order spread-of-plasticity approach for nonlinear time-history analysis of space semi-rigid steel frames", Finite Elements in Analysis and Design, 105:1-15. https://doi.org/10.1016/j.finel.2015.06.006

[7] Chiorean, C.G. (2010), "Computerised interaction diagrams and moment capacity contours for composite steel-concrete cross-sections", Eng. Struc., 32; 3734-3757. https://doi.org/10.1016/i.engstruct.2010.08.019

[8] Liew, J. R., Chen, H., and Shanmugam, N. E. (2001), "Inelastic analysis of steel frames with composite beams", Journal of Structural Engineering, 127(2), 194-202. https://doi.org/10.1016/S0141-0296(99)00085-1

[9] Iu, C. K., Bradford, M.A., \& Chen, W.F. (2009), "Second-order inelastic analysis of composite framed structures based on the refined plastic hinge method", Eng. Struc., 31(3), 799-813. https://doi.org/10.1016/j.engstruct.2008.12.007

[10] Chiorean, C.G. (2013), "A computer method for nonlinear inelastic analysis of 3D composite $\begin{array}{llll}\text { steel-concrete frame } & \text { Structures", Eng. } & \text { Struc., 57, } & \end{array}$ https://doi.org/10.1016/i.engstruct. 2013.09.025

[11] Liew, J.R., Chen, H., Shanmugam, N.E. and Chen, W.F. (2000), "Improved nonlinear plastic hinge analysis of space frame structures", Eng. Struc., 22(10), pp.1324-1338. https://doi.org/10.1016/S0141-0296(99)00085-1

[12] Kim, S.E., Kim, Y. and Choi, S.H. (2001a), "Nonlinear analysis of 3-D steel frames", Thinwalled structures, 39(6), pp.445-461. https://doi.org/10.1016/S0263-8231(01)00017-9

[13] Kim, S.E., Park, M.H. and Choi, S.H., (2001b), "Direct design of three-dimensional frames using practical advanced analysis", Eng. Struc., 23(11), pp.1491-1502. https://doi.org/10.1016/S0141-0296(01)00041-4

[14] Kim, S.E. and Choi, S.H., (2001), "Practical advanced analysis for semi-rigid space frames", International journal of solids and structures, 38(50-51), pp.9111-9131. https://doi.org/10.1016/S0020-7683(01)00141-X

[15] Skordeli, M.A. and Bisbos, C.D., (2010), "Limit and shakedown analysis of 3D steel frames via approximate ellipsoidal yield surfaces", Eng. Struc., 32(6), pp.1556-1567. https://doi.org/10.1016/j.engstruct.2010.02.004 


\title{
EXPERIMENTAL STUDY OF RESILIENT PREFABRICATED STEEL FRAME WITH ALL-BOLTED BEAM-TO-COLUMN CONNECTIONS
}

\author{
Ai-Lin Zhang ${ }^{1,2,3}$, Guang-Hao Shangguan ${ }^{3}$, Yan-Xia Zhang ${ }^{1,3,{ }^{*}}$, Qing-Bo Wang ${ }^{3}$ and Wen-Chao Cai ${ }^{3}$ \\ Beijing Advanced Innovation Center for Future Urban Design, Beijing University of Civil Engineering and Architecture, Beijing, China \\ ${ }^{2}$ Beijing Engineering Research Center of High-rise and Large-span Prestressed Steel Structure, Beijing University of Technology, Beijing, China \\ ${ }^{3}$ School of Civil and Transportation Engineering, Beijing University of Civil Engineering and Architecture, Beijing, China \\ *(Corresponding author: E-mail: zhangyanxia@bucea.edu.cn)
}

\section{A B S T R A C T}

To avoid welding on a construction site, this paper proposes a novel resilient prefabricated steel frame with all-bolted beamto-column connections (ABRPSF), which is optimized based on a resilient prefabricated steel frame with mixed welded and bolted beam-to-column connections (WBRPSF) that was proposed and investigated in a previous study. Four-story, 3 $\times 5$ bay prototype structures of the ABRPSF and WBRPSF are designed, and their substructures are used to conduct pseudodynamic tests, whose results are then compared to investigate the seismic behavior of ABRPSFs. The results indicate that the seismic behavior and recentering capacity of a new structural system composed of ABRPSFs are similar to those of WBRPSFs; in addition, the ABRPSF improves the assembly efficiency significantly and can be adopted as a reliable alternative to the WBRPSF.
ART I CLE H I S T O RY

$\begin{array}{ll}\text { Received: } & \text { 18 September } 2019 \\ \text { Revised: } & \text { 12 June 2020 } \\ \text { Accepted: } & \text { 22 June 2020 }\end{array}$

\section{KE Y W O R D S}

Resilient prefabricated steel frame All-bolted beam-to-column connection;

Mixed welded and bolted beam-tocolumn connection; Pseudo-dynamic test; Seismic behavior

\section{Introduction}

Compared with conventional moment-resisting steel frames, self-centering steel moment-resisting frames (SC-MRFs) exhibit better performances in controlling structural damage, absorbing seismic energy through plastic deformation of energy dissipation devices, and rehabilitating the initial function of a steel frame following intensive seismic actions. In the past decades, this new structural system has been integrated significantly by researchers at worldwide. Early experimental studies pertaining to SC-MRFs were initiated by Ricles et al. [1, 2]. Subsequently, these studies were extended to novel configurations and improvements in energy dissipation devices, for which representative studies based on energy dissipating bars were performed by Christopoulos et al. [3], those based on bolted web friction devices were performed by Tsai et al. [4], and those based on bottom flange friction damped devices were performed by P. Rojas et al. [5] and Wolski et al. [6]. Additionally, Khoo et al. [7] proposed a self-centering sliding hinge joint to achieve the intended function by mainly relying on friction ring springs. Garlock et al. [8] conducted inelastic cyclic loading tests of six full-scale post-tensioned (PT) steel connection with bolted top and seat angles and proposed closed-form expressions. Lin et al. [9] proposed a steel SC-MRF, conducted quasistatic pushover tests under maximum considered earthquake ground motions, and investigated the performance of the SC-MRF under an earthquake design basis [10]. Georgios et al. [11] proposed a computational framework for the automated seismic design of SC-MRFs based on experimental results. Vasdravellis et al. [12] proposed a new self-centering steel PT connection using high-strength steel PT bars to induce self-centering behavior and cylindrical pins with an hourglass shape to provide an enhanced deformation capacity. Steel structures constructed using shape memory alloys for achieving self-centering capabilities have been widely investigated [13-17]. Meanwhile, innovative structural configurations of self-centering steel braces in steel frames have been designed and corresponding tests have been conducted [18-22]. Based on the abovementioned studies, Zhang et al. proposed novel self-centering prefabricated steel frames [23-24], resilient prefabricated steel frames [25], and prefabricated beam-column connections with short strands in a self-centering steel frame [26]; these structures not only demonstrate advantageous seismic and recentering performances, but also avoid the potential issue of aerial pretension in the aforementioned beam-through self-centering steel frames.

Herein, we propose an original all-bolted beam-to-column connection resilient prefabricated steel frame (ABRPSF) based on previous studies [23-26]. This new steel frame has an improved beam-to-column connection, which is better than welded beam-to-column connection resilient prefabricated steel frames (WBRPSFs) [24]. In ABRPSFs, all-bolted connections are achieved using L-plates and high-strength bolts to avoiding potential issues from on-site welding. Structural configurations of ABRPSFs and WBRPSFs have been investigated, and pseudo-dynamic tests have been performed on both steel frames to compare and investigate their seismic performances and recentering capacities. Results indicated that the deformation performance, gap-opening mechanism, PT force variation, and strain development conditions of the ABRPSF are similar to those of the WBRPSF, whereas its energy dissipation capacity is more favorable. The ABRPSF not only possesses the advantages of the WBRPSF, but also improves the assembly efficiency by avoiding welding.

\section{Details of WBRPSF and ABRPSF}

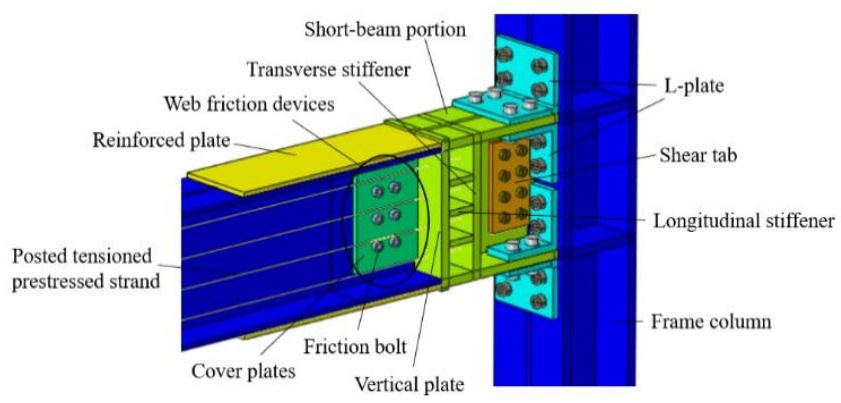

Fig. 1 Construction details of ABRPSF

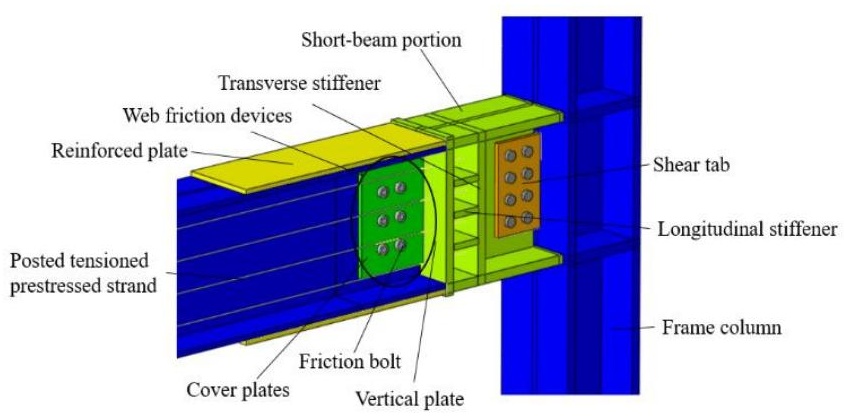

Fig. 2 Construction details of WBRPSF

Fig. 1 shows the structural details of the ABRPSF. The frame column and the assembled prestressed beam were connected by L-plates and high-strength bolts, which were used to connect the beam and column flange as well as the bolt beam web and shear tab that were welded to the column flange. As shown 
in Fig. 1, the entire beam assembly was primarily composed of steel strands as well as short and long beam portions. The steel strands were arranged parallel to the beam length direction and prestressed to connect two short beam portions and a long beam portion. Each short beam portion included an I-section beam, a vertical plate, transverse stiffeners, and longitudinal stiffeners. The vertical plate was reliably welded to the internal end of the I-section beam to provide a close and stable contact with the long beam portion. Transverse stiffeners were welded to the I-section beam web as the anchor end of the steel strands; additionally, small-sized longitudinal stiffeners were welded to the surfaces of the transverse stiffeners, I-section beam web, and vertical plate to strengthen the anchored regions of the transverse stiffeners. The long beam portion mainly comprised an I-section beam, reinforced plates, and web friction devices. The reinforced plate was welded to the outside surface of the beam end flange to avoid local buckling. The web friction devices comprised elongated holes that were opened on the beam web to allow the slippage of high-strength bolts, cover plates welded on the vertical plate in advance, and a brass plate sandwiched between the cover plate and I-section beam web to ensure a stable friction behavior.

Fig. 2 shows the structural details of the WBRPSF, which are identical to

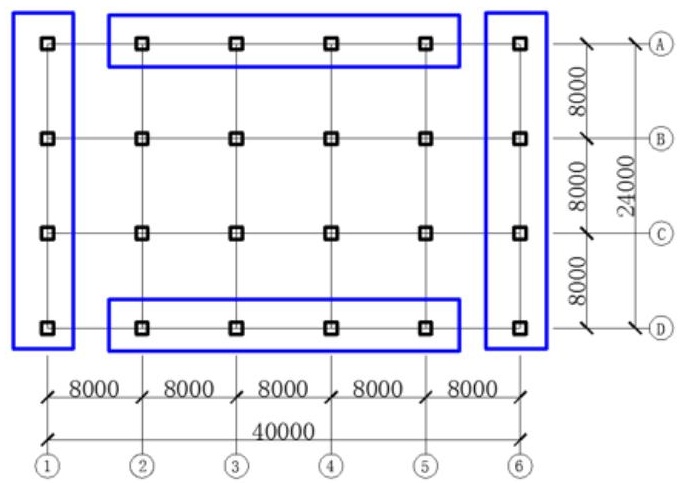

Fig. 3 Layout of prototype structure of both steel frames

\subsection{Substructure}

To simplify the pseudo-dynamic test and minimize negative effects on the test results, a single span of steel frame was selected as a substructure, as shown in Fig. 4, in which the first story was designed as a testing substructure, whereas other stories constituted the computing substructure. Considering the limited conditions of the laboratory, a 0.75 scale of the prototype structure was adopted to conduct the test, in which the frame column sections was determined by adopting the same axial compression ratio with the corresponding columns in the prototype structure. The section dimensions of the columns, short beam portions, and long beam portions were $\mathrm{H} 300 \times 300 \times 20 \times 30, \mathrm{H} 482 \times 250 \times 18$ $\times 30$, and $\mathrm{H} 450 \times 250 \times 14 \times 16$, respectively. The column web stiffeners at the beam-to-column panel zone, located at the level altitude with beam flanges, was designed to be $30 \mathrm{~mm}$ thick to avoid local buckling at the contact area of the column flange. The transverse and longitudinal stiffeners installed at the short beam portions were $30 \mathrm{~mm}$ and $20 \mathrm{~mm}$ thick, respectively. The vertical plate between the short and long beam portions was $30 \mathrm{~mm}$ thick. Additionally, six M24 (with a nominal diameter of $24 \mathrm{~mm}$ ) 10.9s high-strength bolts were adopted in the web friction devices and eight M20, 10.9s [27] high-strength those of the ABRPSF except the beam-to-column connection. Unlike the ABRPSF, the frame column and the assembled prestressed beam were connected by welding the beam to the column flanges as well as the bolt beam webs to the shear tabs that were welded to the column flange. The entire beam assembly, similar to the prestressed steel beam, was proposed by our research group [24].

\section{Prototype structure and substructure}

\subsection{Prototype structure}

Four-story, $3 \times 5$ prototype structures of the WBRPSF and ABRPSF were designed, and the plane layout is shown in Fig. 3; meanwhile, the substructure schematics of both steel frames are shown in Fig. 4. The first story was $3.9 \mathrm{~m}$ high, whereas the second to fourth story was $3.6 \mathrm{~m}$ high. Each story was $8 \mathrm{~m}$ long, and the steel frames highlighted by blue brackets were designed as a WBRPSF or an ABRPSF. The section dimensions of the frame column and frame beam were $\mathrm{H} 400 \times 400 \times 34 \times 34$ and $\mathrm{H} 588 \times 300 \times 12 \times 20$, respectively.

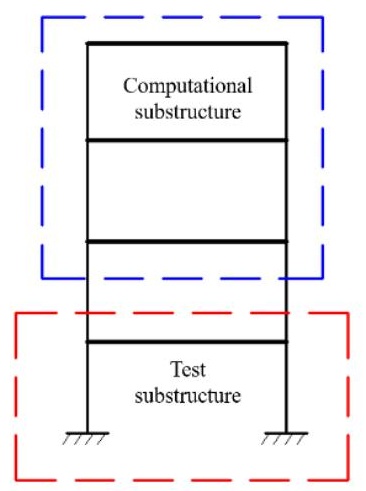

Fig. 4 Substructure schematics of both steel frame

bolts were used in the beam-to-column connection. Each steel strand was composed of 19 steel wires that functioned comprehensively with a nominal diameter of $21.8 \mathrm{~mm}$, nominal section area of $312.9 \mathrm{~mm}^{2}$, and nominal ultimate tensile strength of $T_{u}=1860 \mathrm{~N} / \mathrm{mm}^{2}$; furthermore, the wires were prestressed to $0.25 \mathrm{~T}_{\mathrm{u}}$ (we set the initial PT force as $\mathrm{T}_{0}$ ). For the ABRPSF in particular, two large L-shaped plates $(\mathrm{L} 200 \times 250 \times 24)$, four small L-shaped plates (L200 $\times$ $95 \times 24$ ), and 24 M24 high-strength bolts were additionally adopted to ensure a reliable connection between the beam flange and column flange.

\subsection{Material properties}

Q345B steel was adopted for both the WBRPSF and ABRPSF, and the material properties obtained from the completed material tests are listed in Table 1. The steel strands used in the test specimens remained in the elastic state during the entire loading process. Three group material tests of steel strands were conducted, and their properties are listed in Table 2. The experimental results show that the friction coefficient between the brass plate $(3 \mathrm{~mm})$ and steel plate was 0.34 .

\section{Table 1}

Tensile testing results of standard samples

\begin{tabular}{cccccc}
\hline Thickness $(\mathrm{mm})$ & Yield strength $(\mathrm{MPa})$ & Ultimate strength(MPa) & Percent elongation at fracture $(\%)$ & Elastic modulus $\times 10^{5}(\mathrm{MPa})$ & Ratio of tensile and yield strength \\
\hline 14 & 384 & 561 & 27.0 & 2.15 & 1.46 \\
16 & 392 & 555 & 23.3 & 2.06 & 1.42 \\
18 & 381 & 555 & 25.3 & 2.22 & 1.46 \\
20 & 384 & 550 & 25.7 & 2.09 & 1.43 \\
22 & 388 & 574 & 26.8 & 2.09 & 1.48 \\
30 & 350 & 505 & 26.5 & 2.07 & 1.44 \\
\hline
\end{tabular}


Table 2

Prestressed strand material properties

\begin{tabular}{|c|c|c|c|c|}
\hline Strand & Specimen & Yield strength (MPa) & Ultimate strength (MPa) & Elastic modulus (GPa) \\
\hline & 1 & 1728.3 & 1894.5 & 203 \\
\hline $1 \times 19$ & 2 & 1727.1 & 1895.8 & 205 \\
\hline \multirow[t]{2}{*}{$1860 \mathrm{MPa}$} & 3 & 1732.8 & 1875.4 & 200 \\
\hline & average value & 1729 & 1889 & 203 \\
\hline
\end{tabular}

\section{Pseudo-dynamic test}

\subsection{Testing setup and loading devices}

The testing setup schematics of the pseudo-dynamic test are shown in Fig. 5; the multistory structural remote cooperative pseudo-dynamic test platform [24] was used to complete the loading process. Seismic actions were performed on the steel frame using a 200t lateral actuator attached to the reaction wall. Two end vertical actuators were fixed on the reaction frame, and they were placed on the top of both columns to simulate the axial forces transferred from the upperfloor columns; a middle vertical actuator and a force transfer girder were adopted to simulate the live load transferred from the upper floor. Both column feet were fixed on the column bases, whose six degrees-of freedom were restrained by adopting both pressure beams. Moreover, two lateral supports were installed to avoid the possible out-plane instability of the steel frame. Earthquake ground motion records were input to the platform structure software to calculate the corresponding displacement, which was then used to control the lateral actuator. To achieve this, the platform structure must be input to the floor quality and theoretical interstory restoring force model, similar to that investigated in a previous study [24]. The photographs of the ABRPSF and WBRPSF are shown in Figs. 6 and 7, respectively.

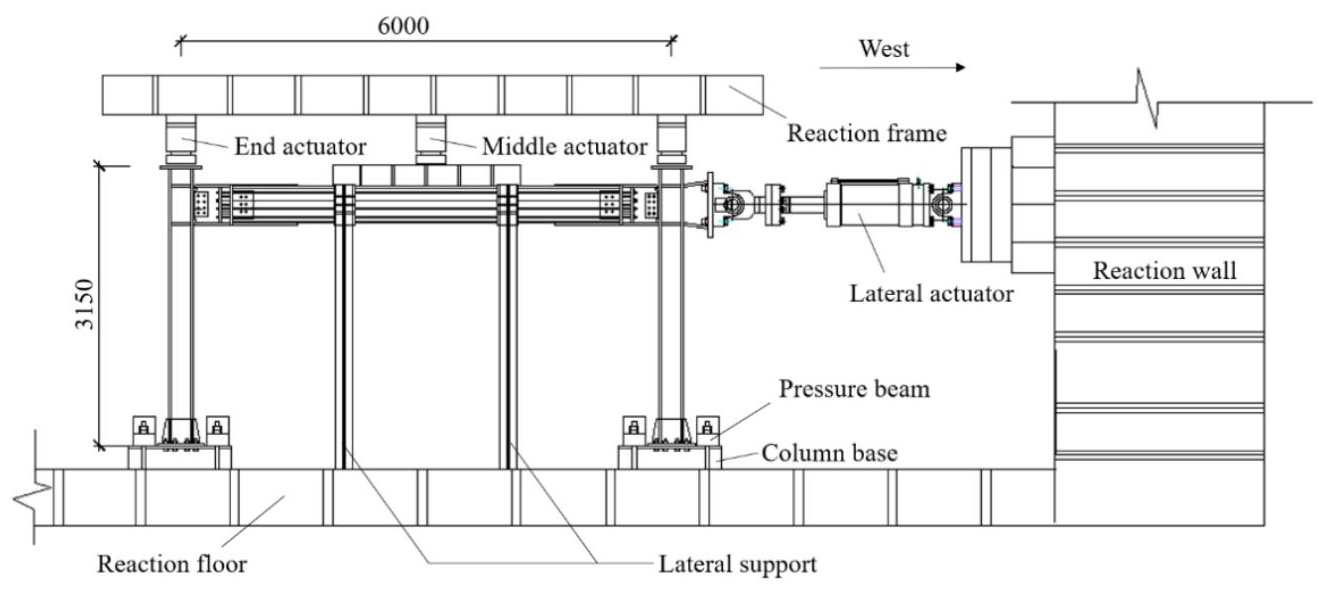

Fig. 5 Test setup schematic

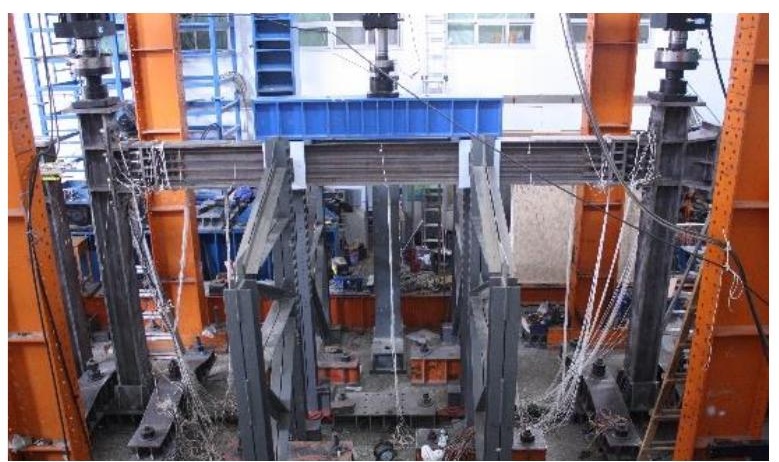

Fig. 6 Photograph of ABRPSF

\subsection{Selection of earthquake ground motions}

Ground motions from the EL-Centro and Wenchuan earthquakes were selected to conduct the pseudo-dynamic test; the time history curves of the earthquake accelerations for the ground motion records are shown in Figs. 8 and 9, where the peak ground accelerations (PGAs) are modified to $0.4 \mathrm{~g}$. The acceleration response spectrums of the EL-Centro, Wenchuan, and standard ground motion records are shown in Fig. 10. The result of the structural computation shows that the first period of the prototype structure was $1.23 \mathrm{~s}$, and a damping ratio of 0.05 was used according to GB50011-2016 [28].

Both earthquake ground motions were input into the testing equipment

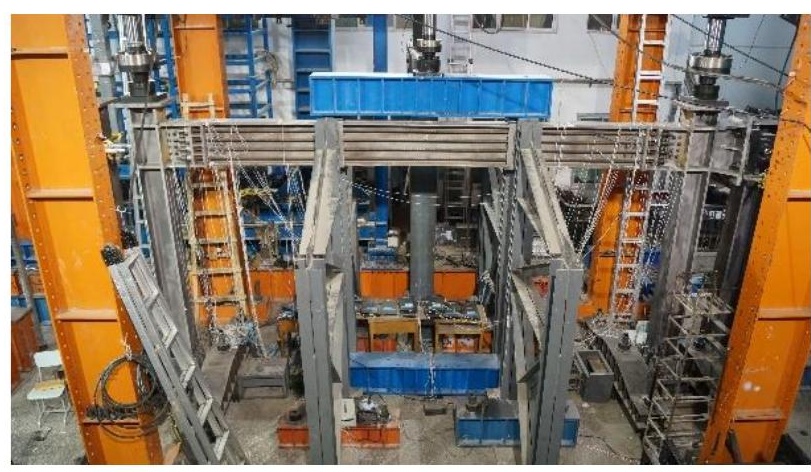

Fig. 7 Photograph of WBRPSF

under various peak ground accelerations, i.e., 0.07, 0.20, 0.40, and 0.51g, which corresponded to four seismic levels, i.e., 8-degree frequent earthquake, 8-degree fortification earthquake, 8-degree rare earthquake, and 8.5-degree rare earthquake, as described in GB50011-2016 [28]. The ground motion records of Wenchuan with PGAs of $0.62,0.81,1.0$, and $1.2 \mathrm{~g}$ were input into the testing equipment to study the seismic performance of both steel frames under higher seismic levels. 


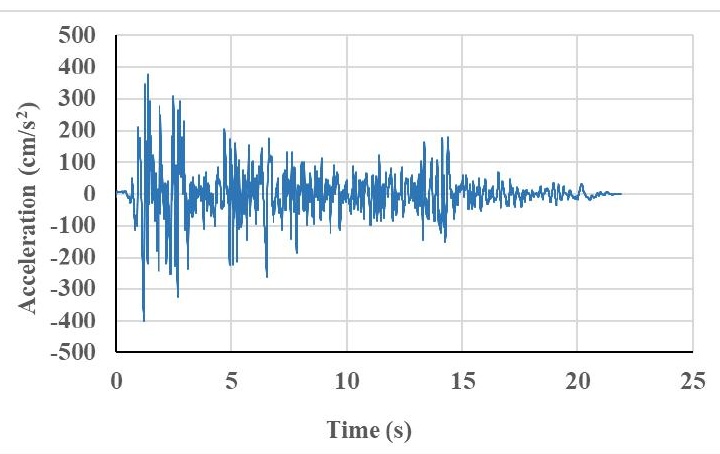

Fig. 8 EL-Centro ground motion record

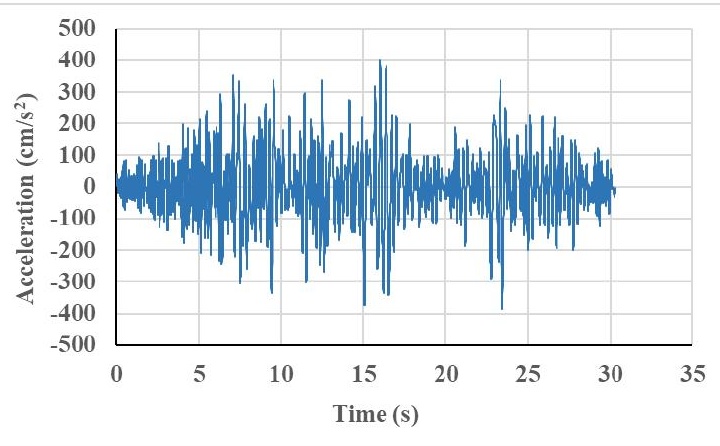

Fig. 9 Wenchuan ground motion record

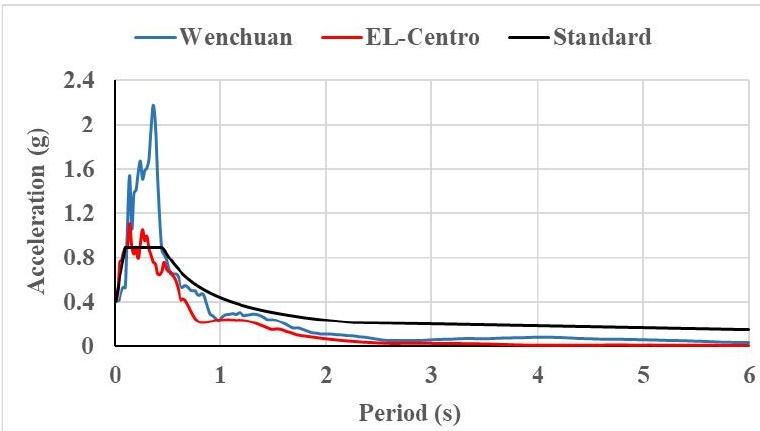

Fig. 10 Acceleration response spectrum comparison

\subsection{Measurement devices}

Fig. 11 shows the arrangement of various measurement instruments for the ABRPSF. Strain gauges were fixed to measure the strain level of typical positions, such as the column foot web and flange, column panel zone, beam reinforced plate, beam flange close to column, L-plate, vertical plate, and beam web. Furthermore, three-directional strain gauges were adopted on the area with complex stress distribution conditions. The PT forces of eight steel strands were monitored using pressure sensors with a range of $250 \mathrm{kN}$. Additionally, eight small displacement meters were installed to measure the gap opening size. The actuator force was monitored using a built-in sensor.

Fig. 12 shows the arrangements of various measurement instruments for the WBRPSF, which are identical to those for the ABRPSF except for the strain gauges on the L-plates.
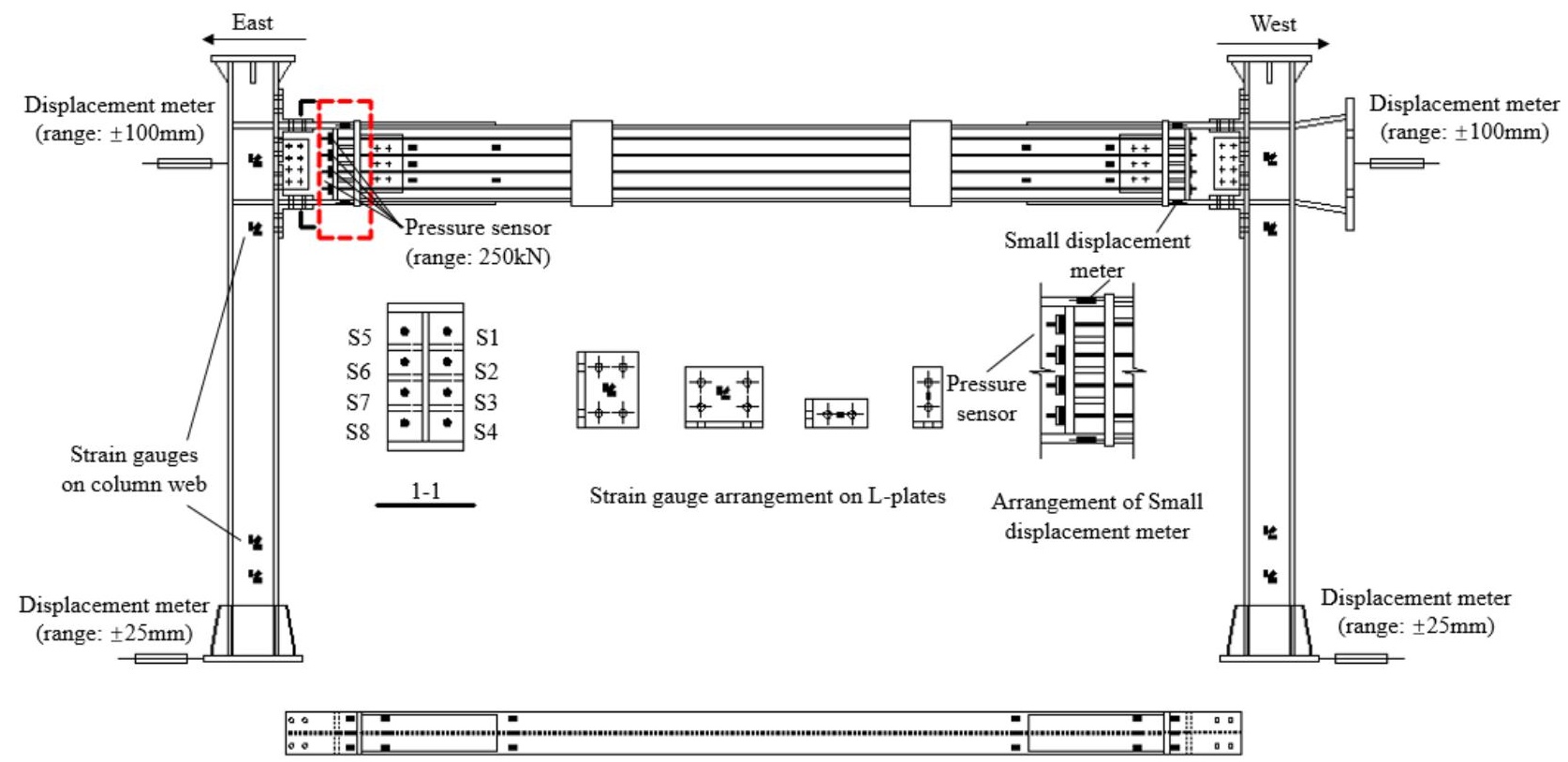

Strain gauge arrangement on upper flange of the assembled beam

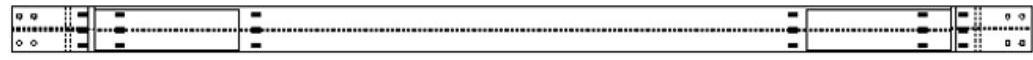

Strain gauge arrangement on lower flange of the assembled beam

Fig. 11 Arrangement of measurement devices for ABRPSF 

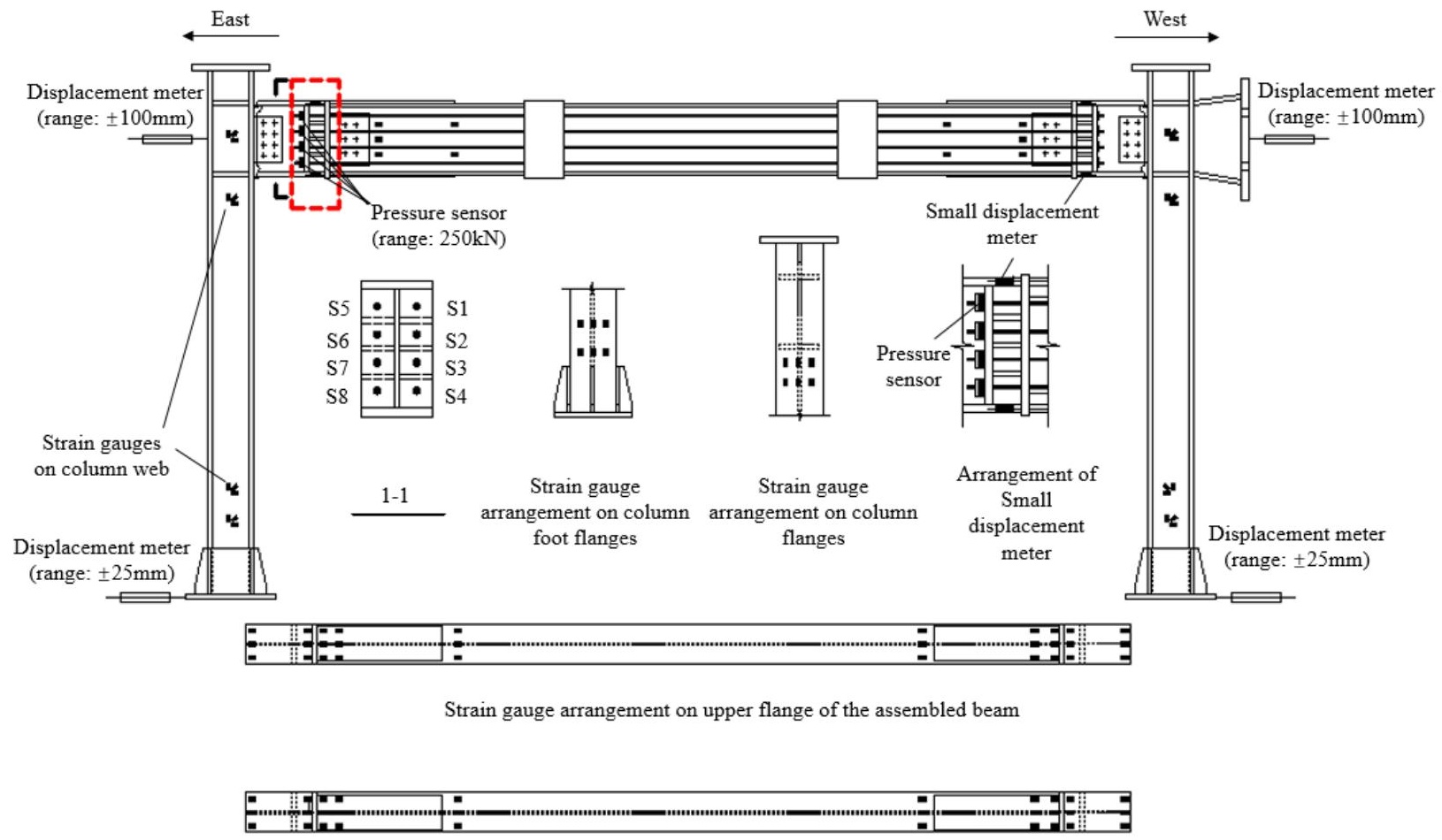

Strain gauge arrangement on lower flange of the assembled beam

Fig. 12 Arrangement of measurement devices for WBRPSF

\section{Comparison of Testing Results of ABRPSF and WBRPSF}

During testing, both EL-Centro and Wenchuan seismic records were adopted to excite the both steel frames. When the seismic level was under a PGA of $0.62 \mathrm{~g}$, the ABRPSF and WBRPSF were in the elastic state; therefore, when the PGA exceeded $0.62 \mathrm{~g}$, a certain degree of plasticity developed in the steel frames. The design goal that we set was "no gap opening and no damage occurs during frequent earthquakes, gap openings are formed to dissipate energy, and no damage occurs to the main structure under fortification earthquake action, i.e., only extremely small structural damages occur and the structure can still operate normally during rare or extremely rare earthquakes." Subsequently, only the Wenchuan seismic record was used to conduct the excitation process.

\subsection{Deformation}

The displacement responses of the ABRPSF and WBRPSF under various seismic levels of the EL-Centro seismic record are shown in Figs. 13(a)-(d), which reveal similar variation trends for both structural systems. Table 3 shows the maximum displacement responses and the corresponding story drifts for the ABRPSF and WBRPSF under the EL-Centro seismic records. When PGA =
$0.07 \mathrm{~g}$, the maximum displacements for the ABRPSF and WBRPSF were still relatively low, i.e., 4.95 and $3.74 \mathrm{~mm}$, respectively, and the corresponding story drifts were 1/594 and 1/786 rad, respectively, under a PGA of $0.20 \mathrm{~g}$. Meanwhile, the maximum displacements for the ABRPSF and WBRPSF were was 13.34, and $9.37 \mathrm{~mm}$, respectively, whereas their story drifts were $1 / 220$ and $1 / 334 \mathrm{rad}$, respectively. Under rare earthquake motions, the displacement responses for both steel frames were relatively larger. When PGA $=0.40 \mathrm{~g}$, the displacements of the ABRPSF and WBRPSF were 20.4 and $18.06 \mathrm{~mm}$, respectively, whereas their story drifts were 1/144 and 1/163 rad, respectively. Meanwhile, when PGA $=0.51 \mathrm{~g}$, the displacements of the ABRPSF and WBRPSF were 26.6 and 23.36 $\mathrm{mm}$, respectively, whereas their story drifts were $1 / 110$ and $1 / 126 \mathrm{rad}$, respectively. We observed that the displacement response of the ABRPSF increased slightly compared with that of the WBRPSF, indicating that the lateral rigidity of the ABRPSF was smaller than that of the WBRPSF. When PGA = $0.51 \mathrm{~g}$, the maximum story drift was $1 / 110$ and $1 / 126 \mathrm{rad}$ for the ABRPSF and WBRPSF, respectively, whereas the maximum story drifts of both steel frames did not exceed the elastic story drift limit of a frame defined in the Code for Seismic Design of Buildings (1/50); this implies that the structure was safe, and that both frames can return to their initial positions after an earthquake.

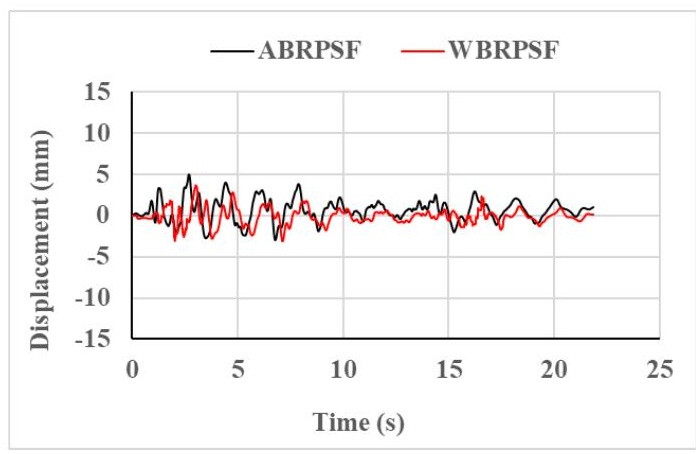

(a) $\mathrm{PGA}=0.07 \mathrm{~g}$

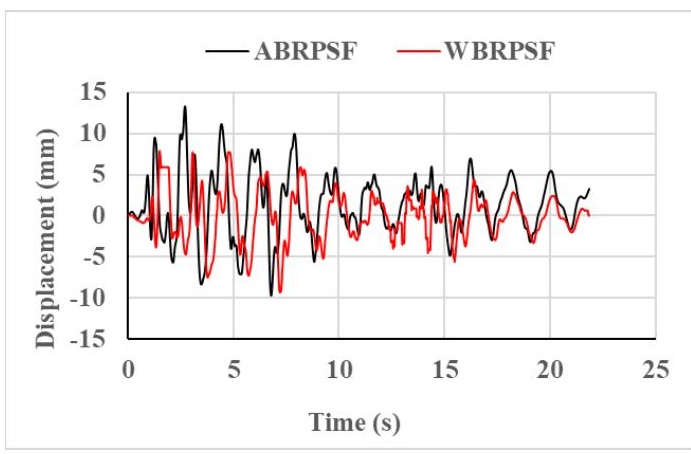

(b) $\mathrm{PGA}=0.20 \mathrm{~g}$ 


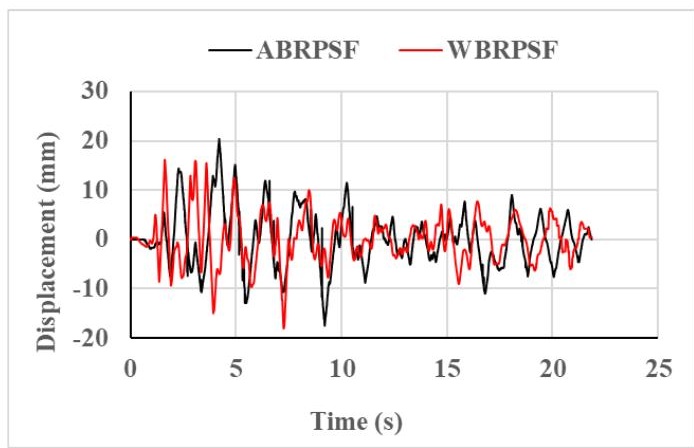

(c) $\mathrm{PGA}=0.40 \mathrm{~g}$

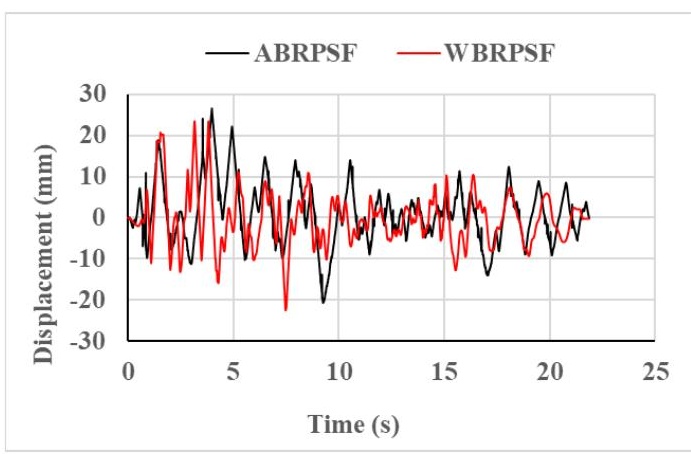

(d) $\mathrm{PGA}=0.51 \mathrm{~g}$

Fig. 13 Time history curves of displacement response under EL-Centro seismic records

Table 3

Displacements and story drifts of WBRPSF and ABRPSF under various seismic levels

\begin{tabular}{|c|c|c|c|c|c|}
\hline \multicolumn{2}{|c|}{ Seismic event } & \multicolumn{2}{|c|}{ Maximum displacement (mm) } & \multicolumn{2}{|c|}{ Maximum story drift } \\
\hline Ground motion & PGA & ABRPSF & WBRPSF & ABRPSF & WBRPSF \\
\hline \multirow{4}{*}{ EL-Centro } & $0.07 \mathrm{~g}$ & 4.95 & 3.74 & $1 / 594$ & $1 / 786$ \\
\hline & $0.20 \mathrm{~g}$ & 13.34 & 9.37 & $1 / 220$ & $1 / 314$ \\
\hline & $0.40 \mathrm{~g}$ & 20.4 & 18.06 & $1 / 144$ & $1 / 163$ \\
\hline & $0.51 \mathrm{~g}$ & 26.6 & 23.36 & $1 / 110$ & $1 / 126$ \\
\hline \multirow{8}{*}{ Wenchuan } & $0.07 \mathrm{~g}$ & 5.67 & 5.56 & $1 / 518$ & $1 / 529$ \\
\hline & $0.20 \mathrm{~g}$ & 17 & 17.21 & $1 / 173$ & $1 / 171$ \\
\hline & $0.40 \mathrm{~g}$ & 34 & 27.96 & $1 / 86$ & $1 / 105$ \\
\hline & $0.51 \mathrm{~g}$ & 43.4 & 36.37 & $1 / 68$ & $1 / 81$ \\
\hline & $0.62 \mathrm{~g}$ & 52.8 & 45.68 & $1 / 56$ & $1 / 65$ \\
\hline & $0.81 \mathrm{~g}$ & 63 & 52.29 & $1 / 47$ & $1 / 56$ \\
\hline & $1.0 \mathrm{~g}$ & 77.4 & 65.05 & $1 / 38$ & $1 / 45$ \\
\hline & $1.2 \mathrm{~g}$ & 103 & 87.69 & $1 / 29$ & $1 / 34$ \\
\hline
\end{tabular}

Figs. 14(a)-(h) show the displacement responses of both steel frames under the Wenchuan seismic records. From PGA $=0.07 \mathrm{~g}$ to $\mathrm{PGA}=0.40 \mathrm{~g}$, the ABRPSF indicated a larger displacement response than the WBRPSF under the same seismic level because the lateral rigidity of the ABRPSF was smaller than that of the WBRPSF. Meanwhile, the displacement responses of both steel frames showed a significant increase under the Wenchuan records compared with that of the EL-Centro records, as shown in Table 3.

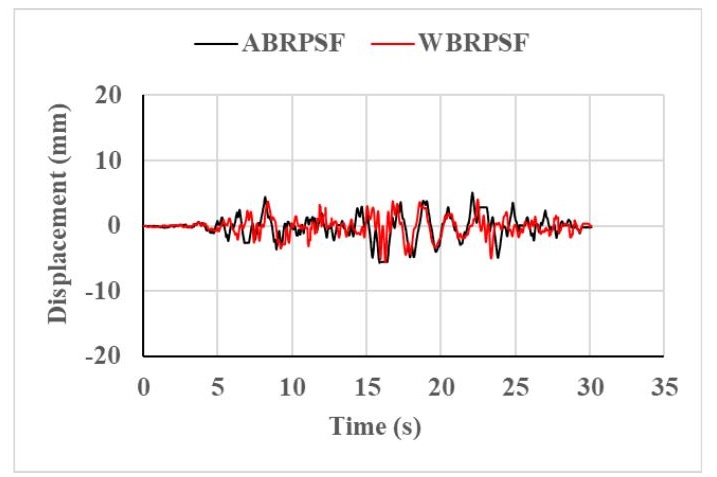

(a) $\mathrm{PGA}=0.07 \mathrm{~g}$

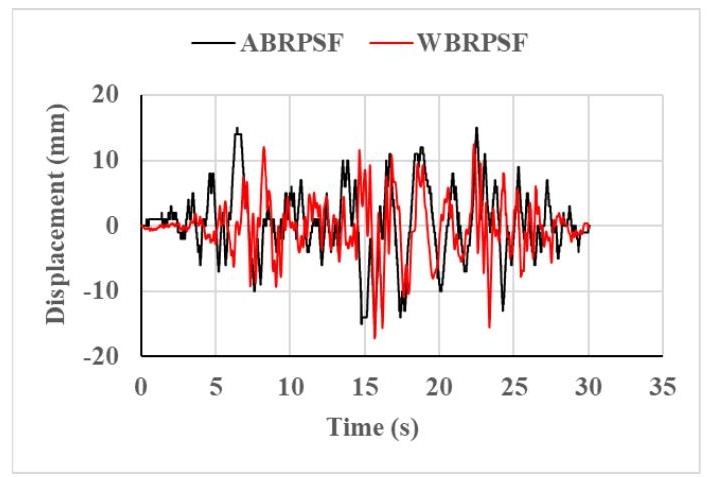

(b) $\mathrm{PGA}=0.20 \mathrm{~g}$ 


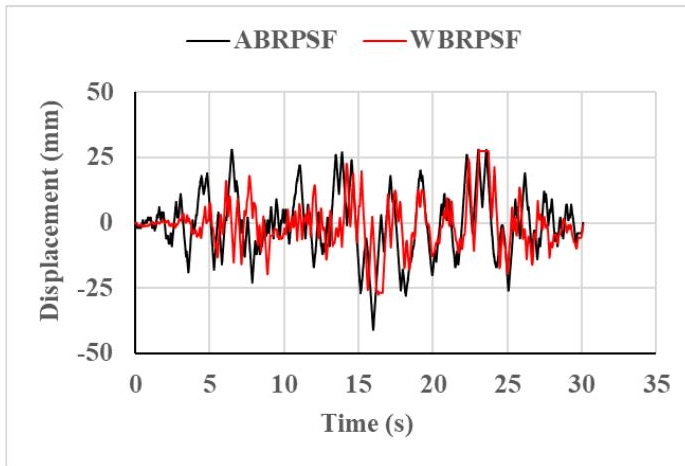

(c) $\mathrm{PGA}=0.40 \mathrm{~g}$

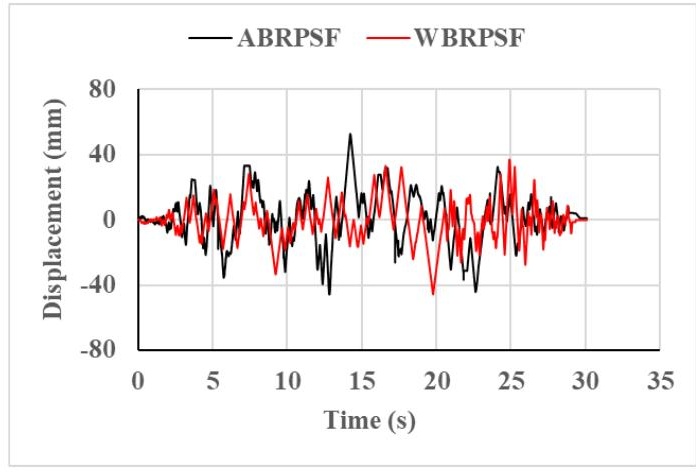

(e) $\mathrm{PGA}=0.62 \mathrm{~g}$

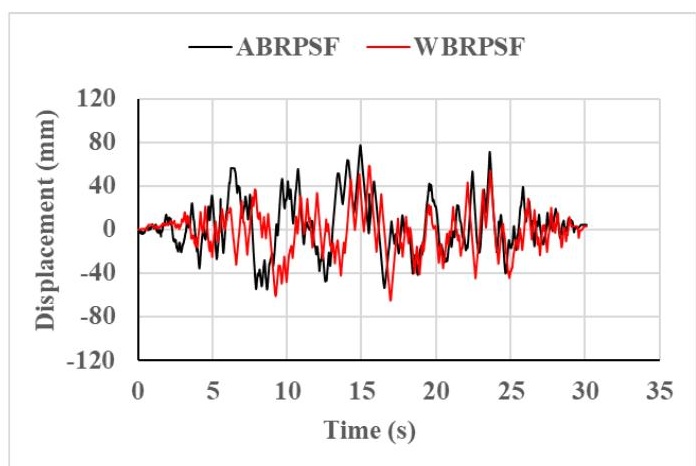

(g) PGA $=1.0 \mathrm{~g}$

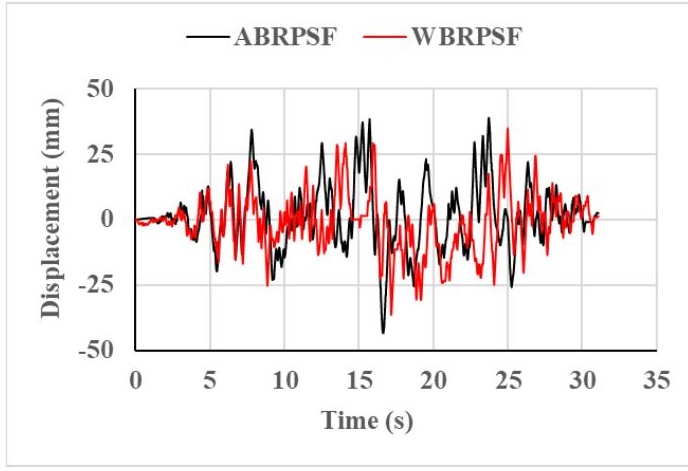

(d) $P G A=0.51 \mathrm{~g}$

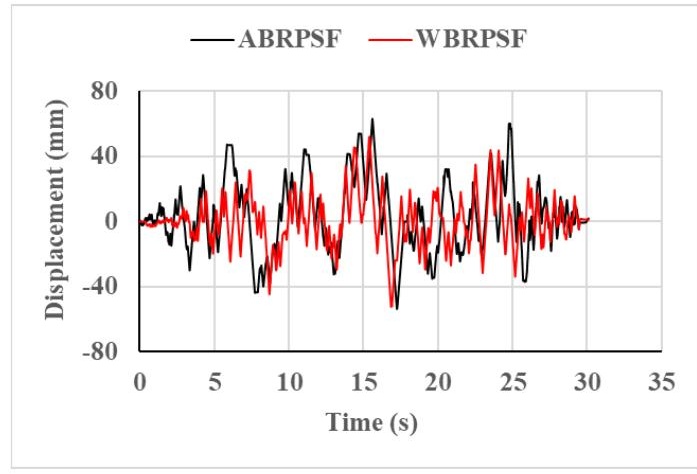

(f) $\mathrm{PGA}=0.81 \mathrm{~g}$

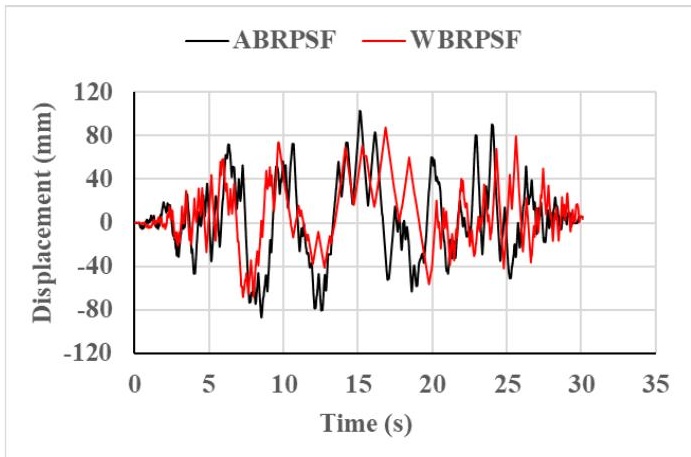

(h) $P G A=1.2 \mathrm{~g}$

Fig. 14 Time history curves of displacement response under Wenchuan seismic records

When the seismic level reached PGA $=0.51 \mathrm{~g}$, as shown in Figs. 14(d)-(h) and Table 3, the variation trends of displacement responses of the ABRPSF and WBRPSF were similar, whereas that of the ABRPSF showed larger values under the same seismic event. To study the seismic performances of both steel frames under higher seismic levels, the ground motion records of Wenchuan with PGAs of $0.62,0.81,1.0$, and $1.2 \mathrm{~g}$ were input to the testing equipment. When PGA $=0.62 \mathrm{~g}$, the maximum displacement responses of the ABRPSF and WBRPSF reached $51.8 \mathrm{~mm}(1 / 56 \mathrm{rad})$ and $45.68 \mathrm{~mm}(1 / 65 \mathrm{rad})$, which revealed a $13.4 \%$ increase in the maximum displacement response of the ABRPSF. When PGA increased to $0.81 \mathrm{~g}$, the maximum displacement responses were $63 \mathrm{~mm}$ $(1 / 47 \mathrm{rad})$ and $52.29 \mathrm{~mm}(1 / 56 \mathrm{rad})$ for the ABRPSF and WBRPSF, respectively, and the corresponding increment was $20.5 \%$, the maximum story drifts exceeded the elastic story drift limit of $1 / 50$, but the frames can return to their original positions after an earthquake. With the increase in the seismic level, the difference between both steel frames fluctuated within $20 \%$. When the PGA reached $1.0 \mathrm{~g}$, the maximum displacements of the ABRPSF and WBRPSF were $77.4 \mathrm{~mm}(1 / 38 \mathrm{rad})$ and $65.05 \mathrm{~mm}(1 / 45 \mathrm{rad})$, respectively. When the PGA increased to $1.2 \mathrm{~g}$, the maximum displacements were $103 \mathrm{~mm}(1 / 29 \mathrm{rad})$ and $87.69 \mathrm{~mm}(1 / 34 \mathrm{rad})$ for the ABRPSF and WBRPSF, respectively, and the growth rates were $18.98 \%$ and $17.46 \%$, respectively. Therefore, it can be concluded that under the EL-Centro and Wenchuan seismic records, the displacement responses for the ABRPSF and WBRPSF reflected a similar variation trend, and the improved ABRPSF indicated a larger response because its lateral rigidity was less than that of the WBRPSF.

\subsection{Gap opening}

Studies regarding the gap opening conditions between the long and short beam portions of the ABRPSF and WBRPSF can be classified into two cases: one is the push direction of the loading equipment and the other is the pull direction. Because the actuator was installed on one end of the steel frame, the asymmetrical loading setup will cause diverse results to the gap opening of the steel frame under different loading directions. All data pertaining to the gap openings were measured using micro displacement meters fixed to the interface between the short and long beam portions. Figs. 15 and 16 show the gap opening photographs of both steel frames in the pull direction under the EL-Centro and Wenchuan seismic records. 


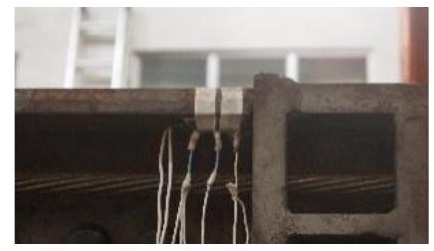

Maximum gap opening rotation $0.04 \%$

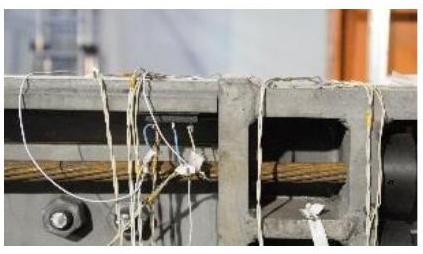

Maximum gap opening rotation $0.03 \%$

(a) $\mathrm{PGA}=0.07 \mathrm{~g}$

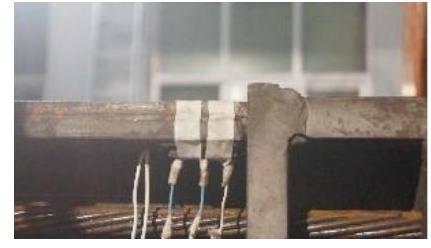

Maximum gap opening rotation $0.14 \%$

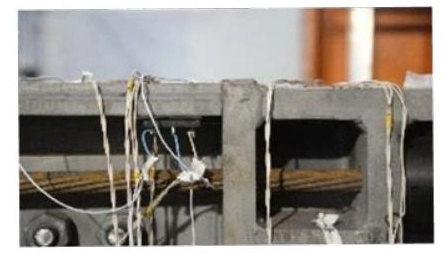

Maximum gap opening rotation $0.21 \%$

ABRPSF

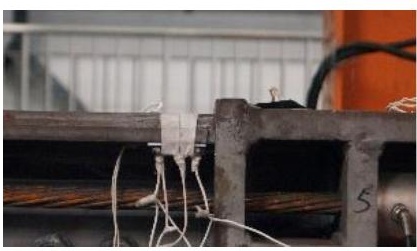

Maximum gap opening rotation $0.06 \%$

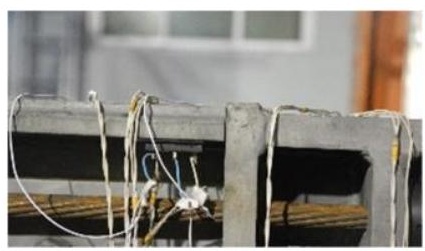

Maximum gap opening rotation $0.07 \%$

ABRPSF

WBRPSF

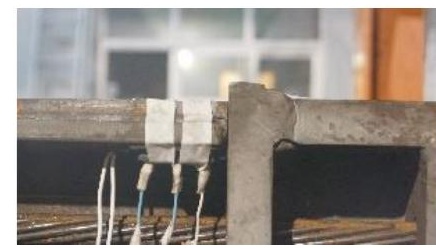

Maximum gap opening rotation $0.16 \%$

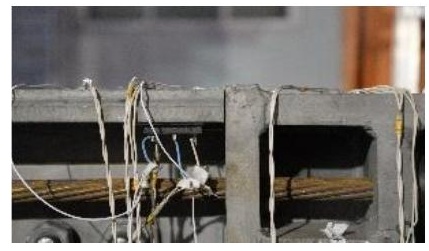

Maximum gap opening rotation $0.32 \%$ WBRPSF

(c) $\mathrm{PGA}=0.40 \mathrm{~g}$

(d) $\mathrm{PGA}=0.51 \mathrm{~g}$

Fig. 15 Gap opening photographs of both steel frames in pull direction under EL-Centro seismic records

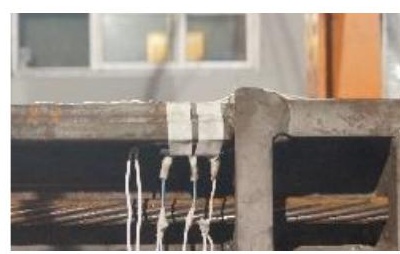

Maximum gap opening rotation $0.03 \%$

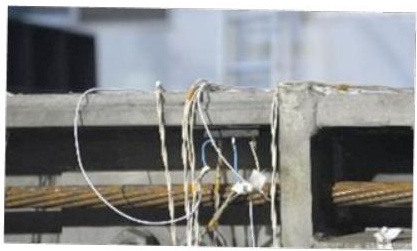

Maximum gap opening rotation $0.05 \%$

(a) $\mathrm{PGA}=0.07 \mathrm{~g}$

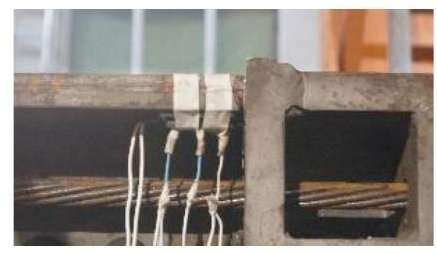

ABRPSF

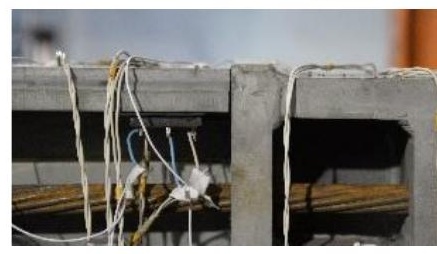

WBRPSF

Maximum gap opening rotation $0.12 \% \quad$ Maximum gap opening rotation $0.38 \%$

(a) $\mathrm{PGA}=0.40 \mathrm{~g}$

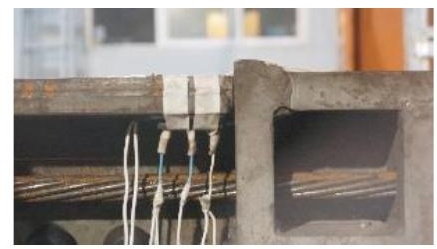

Maximum gap opening rotation $0.38 \%$

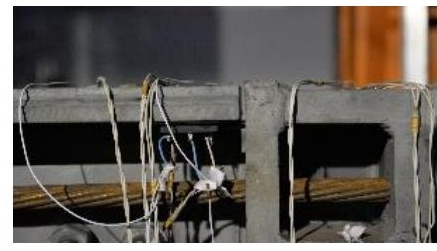

Maximum gap opening rotation $1.23 \%$

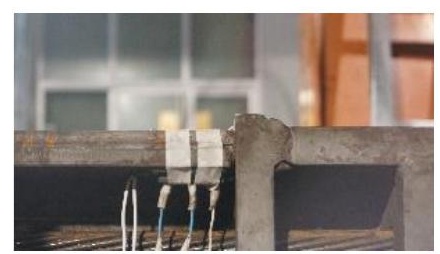

Maximum gap opening rotation $0.12 \%$

ABRPSF

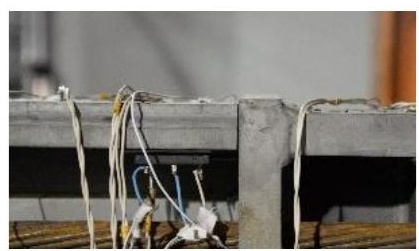

Maximum gap opening rotation $0.21 \%$

WBRPSF

(b) $\mathrm{PGA}=0.20 \mathrm{~g}$

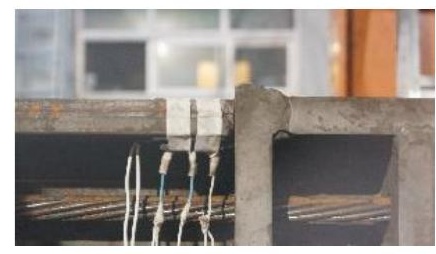

ABRPSF

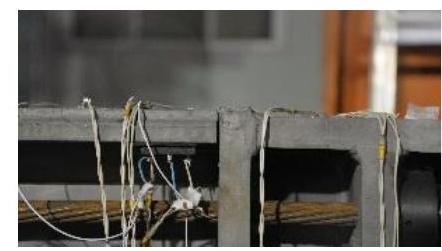

WBRPSF

Maximum gap opening rotation $0.26 \% \quad$ Maximum gap opening rotation $0.58 \%$ (b) $\mathrm{PGA}=0.51 \mathrm{~g}$

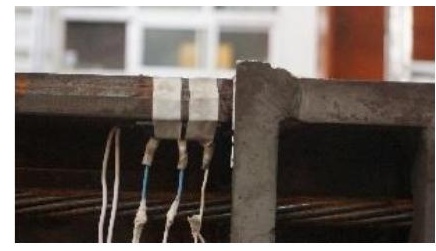

Maximum gap opening rotation $0.40 \%$

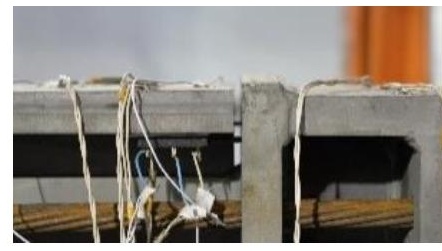

Maximum gap opening rotation $1.38 \%$

ABRPSF 


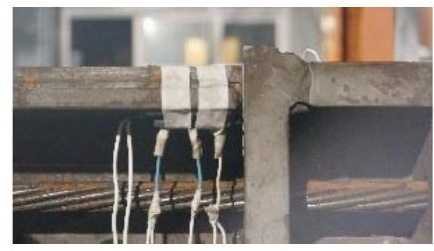

Maximum gap opening rotation $0.45 \%$ ABRPSF

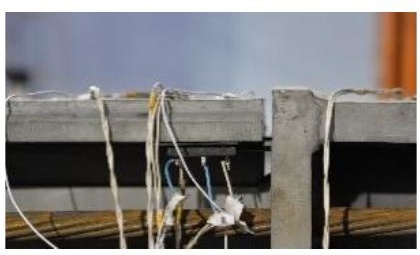

Maximum gap opening rotation 1.68\% WBRPSF

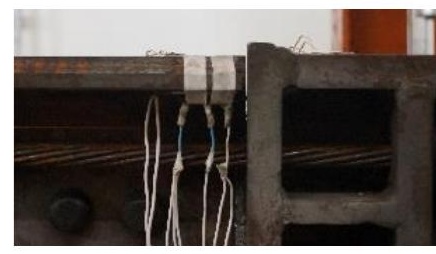

Maximum gap opening rotation $0.48 \%$ ABRPSF

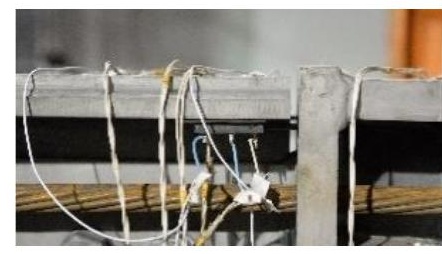

Maximum gap opening rotation $1.96 \%$ WBRPSF

(a) $\mathrm{PGA}=1.0 \mathrm{~g}$

(b) $\mathrm{PGA}=1.2 \mathrm{~g}$

Fig. 16 Gap opening photographs of both steel frames in pull direction under Wenchuan seismic records

The gap opening data of the ABRPSF and WBRPSF under EL-Centro and Wenchuan seismic records are listed in Table 4. As shown, the WBRPSF showed a larger gap opening than the ABRPSF. As the seismic level increased, the increasing trend of the gap opening of the ABRPSF and WBRPSF under the EL-Centro and Wenchuan records became clearer. Under the same seismic level, the gap opening of the ABRPSF was smaller than that of the WBRPSF. The residual gap openings of both steel frames were extremely small, as shown in Table 4.

Therefore, it can be concluded that under the EL-Centro and Wenchuan seismic records, the gap openings of the ABRPSF and WBRPSF showed similar variation trends, and the improved ABRPSF demonstrated a smaller response due to the bolt slippage that occurred on the beam-to-column connection of the
ABRPSF. When the PGA reached $0.62 \mathrm{~g}$, which corresponds to a 9-degree rare earthquake according to the Chinese Code for Seismic Design of Buildings (GB 50011-2010, 2016) under the Wenchuan seismic records, the maximum story drifts of the ABRPSF and WBRPSF reached 1/56 and 1/65 rad, respectively, which were less than the elastic story drift of $1 / 50 \mathrm{rad}$; additionally, the residual gap opening rotation was small, implying that both frames exhibited excellent self-centering capability. When PGA $=1.2 \mathrm{~g}$ under the Wenchuan seismic record, the maximum story drifts of the ABRPSF and WBRPSF were 1/29 and 1/34, respectively, which exceeded the elastic story drift of $1 / 50 \mathrm{rad}$; however, the residual gap opening rotation was $0.00 \%$, indicating that both steel frames had relatively good opening and closing mechanisms and that their self-centering performances were good.

Table 4

Residual gap opening rotation of both steel frames under various seismic levels

\begin{tabular}{|c|c|c|c|c|c|}
\hline \multirow{2}{*}{ Seismic record } & \multirow{2}{*}{ Seismic level } & \multicolumn{2}{|c|}{ Maximum gap opening rotation } & \multicolumn{2}{|c|}{ Residual gap opening rotation } \\
\hline & & ABRPSF & WBRPSF & ABRPSF & WBRPSF \\
\hline \multirow{4}{*}{ EL-Centro } & $\mathrm{PGA}=0.07 \mathrm{~g}$ & $0.04 \%$ & $0.03 \%$ & $0.00 \%$ & $0.00 \%$ \\
\hline & $\mathrm{PGA}=0.20 \mathrm{~g}$ & $0.06 \%$ & $0.07 \%$ & $0.00 \%$ & $0.01 \%$ \\
\hline & $\mathrm{PGA}=0.40 \mathrm{~g}$ & $0.14 \%$ & $0.21 \%$ & $0.01 \%$ & $0.03 \%$ \\
\hline & $\mathrm{PGA}=0.51 \mathrm{~g}$ & $0.16 \%$ & $0.32 \%$ & $0.02 \%$ & $0.07 \%$ \\
\hline \multirow{8}{*}{ Wenchuan } & $\mathrm{PGA}=0.07 \mathrm{~g}$ & $0.03 \%$ & $0.05 \%$ & $0.01 \%$ & $0.00 \%$ \\
\hline & $\mathrm{PGA}=0.20 \mathrm{~g}$ & $0.12 \%$ & $0.21 \%$ & $0.00 \%$ & $0.13 \%$ \\
\hline & $\mathrm{PGA}=0.40 \mathrm{~g}$ & $0.12 \%$ & $0.38 \%$ & $0.00 \%$ & $0.06 \%$ \\
\hline & $\mathrm{PGA}=0.51 \mathrm{~g}$ & $0.26 \%$ & $0.58 \%$ & $0.00 \%$ & $0.02 \%$ \\
\hline & $\mathrm{PGA}=0.62 \mathrm{~g}$ & $0.38 \%$ & $1.23 \%$ & $0.00 \%$ & $0.01 \%$ \\
\hline & $\mathrm{PGA}=0.81 \mathrm{~g}$ & $0.40 \%$ & $1.38 \%$ & $0.00 \%$ & $0.01 \%$ \\
\hline & $\mathrm{PGA}=1.0 \mathrm{~g}$ & $0.45 \%$ & $1.68 \%$ & $0.12 \%$ & $0.02 \%$ \\
\hline & $\mathrm{PGA}=1.2 \mathrm{~g}$ & $0.48 \%$ & $1.96 \%$ & $0.00 \%$ & $0.00 \%$ \\
\hline
\end{tabular}

\subsection{Hysteretic behavior}

Figs. 17(a)-(d) show a comparison of hysteretic curves between the WBRPSF and ABRPSF under the EL-Centro seismic records. Based on the figures, we concluded that the lateral rigidity and carrying capacity of the ABRPSF were slightly lower than those of the WBRPSF, whereas the ductility of the ABRPSF exceeded that of the WBRPSF. When the seismic action reached the frequent earthquake level with $\mathrm{PGA}=0.07 \mathrm{~g}$, the maximum story drifts of the ABRPSF and WBRPSF were 1/594 and 1/786 rad, respectively, which were less than the story drift limit of $1 / 250 \mathrm{rad}$ according to the Code for Seismic Design of Buildings, and the hysteretic curves were linear. When the loading level reached those of fortification earthquakes with PGA $=0.20 \mathrm{~g}$, the maximum story drifts of the ABRPSF and WBRPSF were $1 / 220$ and 1/314, respectively, and hysteretic loops began to appear because the gap opening of both steel frames and the web friction device dissipated energy; consequently, both steel frames were in the elastic state. When the PGA reached 0.40 and $0.51 \mathrm{~g}$, as shown in Figs. 17(c)-(d), clear hysteretic loops appeared for the ABRPSF and WBRPSF. When the PGA reached $0.51 \mathrm{~g}$, the maximum story drifts were $1 / 110$ and $1 / 126 \mathrm{rad}$, which were less than the story drift limit of $1 / 50$ rad according to the Code for Seismic Design of Buildings . The hysteretic loops formed completely, and the energy dissipation was more evident. In each loading process, the structural carrying capacity for the WBRPSF was higher than that for the ABRPSF, but the latter yielded a more satisfying deformation behavior and energy dissipation. Additionally, both frames can return to their original positions after an earthquake, thereby demonstrating their good selfcentering behaviors. 


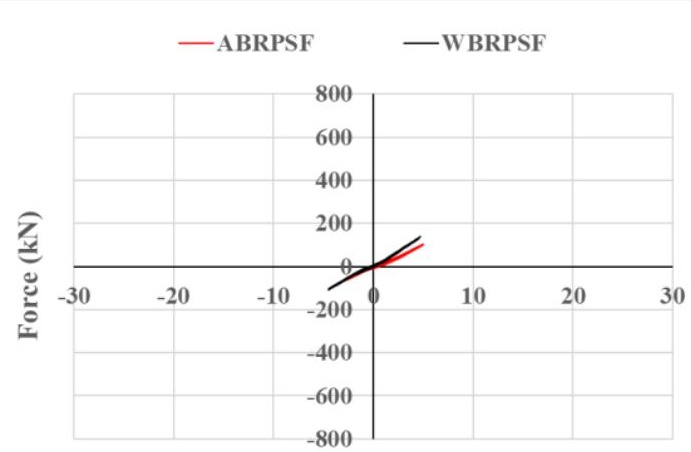

Story drift $(\mathbf{m m})$

(a) $\mathrm{PGA}=0.07 \mathrm{~g}$

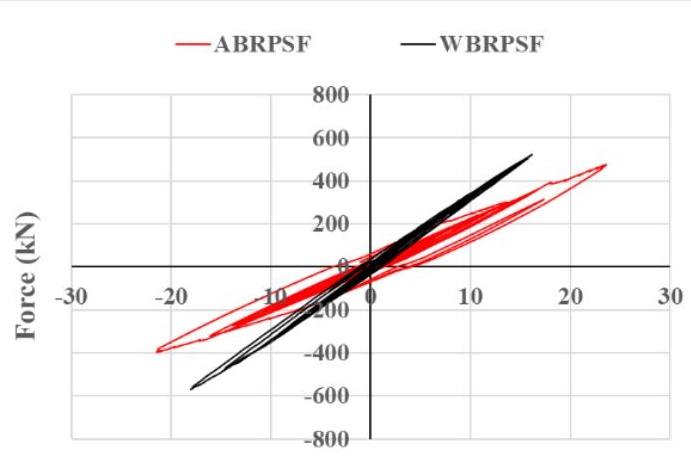

Story drift $(\mathrm{mm})$

(c) $\mathrm{PGA}=0.40 \mathrm{~g}$

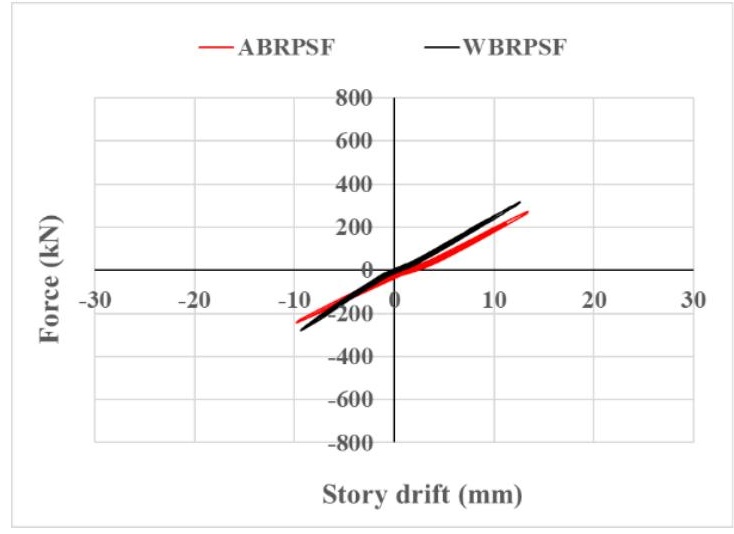

(b) $\mathrm{PGA}=0.20 \mathrm{~g}$

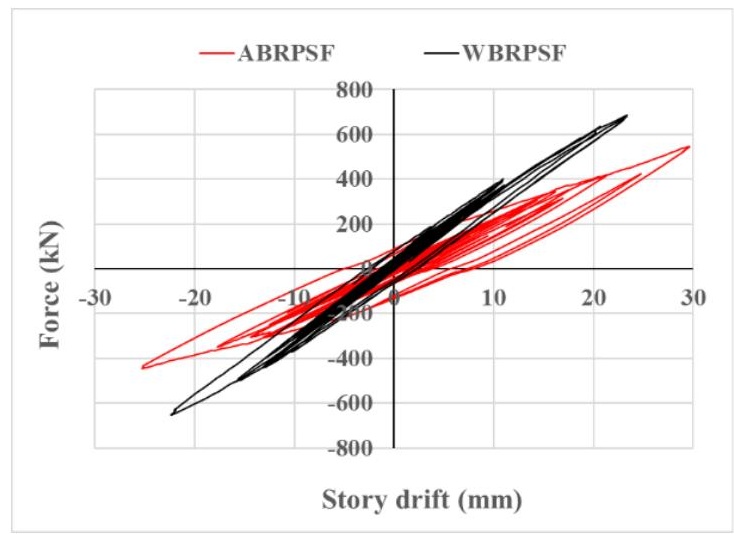

(d) $\mathrm{PGA}=0.51 \mathrm{~g}$

Fig. 17 Hysteretic curves of WBRPSF and ABRPSF under EL-Centro seismic records

Figs. 18(a)-(h) show the hysteretic curves of floor shear and displacement for the ABRPSF and WBRPSF under the Wenchuan seismic records. When PGA $=0.07 \mathrm{~g}$, the maximum story drifts of the ABRPSF and WBRPSF were $1 / 518$ and 1/529 rad, respectively, which were less than the story drift limit of $1 / 250 \mathrm{rad}$ according to the Code for Seismic Design of Buildings . The floor shear and displacement of both steel frames maintained a linear relationship, and the lateral rigidity of the ABRPSF was slightly lower than that of the WBRPSF. When PGA $=0.2 \mathrm{~g}$, the maximum story drifts of the ABRPSF and WBRPSF were $1 / 173$ and $1 / 171 \mathrm{rad}$, respectively, and hysteretic loops began appearing. When the seismic level reached $0.62 \mathrm{~g}$, the maximum story drifts reached 1/56 and 1/65 rad for the ABRPSF and WBRPSF, respectively, which were less than the story drift limit of 1/50 rad according to the Code for Seismic Design of Buildings . The hysteretic loops for both steel frames became more evident. Furthermore, an abrupt change appeared in the hysteretic curves of the WBRPSF, which was mainly caused by the slippage of the column foot. At PGA $=0.81,1.0$, and $1.2 \mathrm{~g}$, the enclosed area of the hysteretic loops for both steel frames continued to increase, and the maximum story drift reached $1 / 29$ and $1 / 34 \mathrm{rad}$ for the ABRPSF and WBRPSF when PGA $=1.2 \mathrm{~g}$, respectively. Although the maximum story drifts exceeded the elastic story drifts limit $(1 / 50$ rad) defined in the Code for Seismic Design of Buildings , both frames can still return to their original positions after an earthquake. Both steel frames demonstrated a satisfactory energy dissipation capacity owing to the web friction devices; however, the ABRPSF demonstrated a more satisfactory energy dissipation capacity because of the energy dispassion of bolt slippage on the beam-to-column connection.

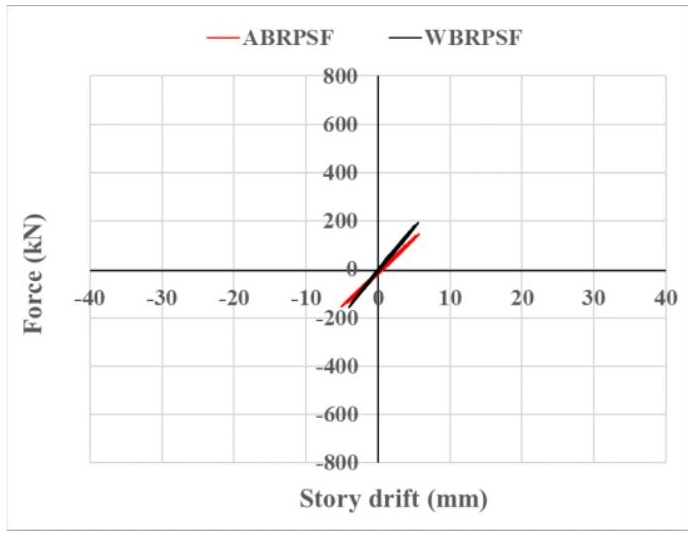

(a) $\mathrm{PGA}=0.07 \mathrm{~g}$

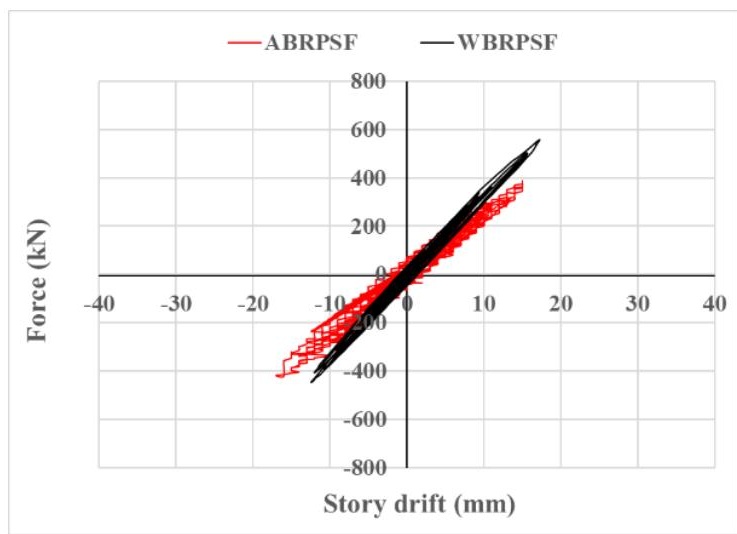

(b) $\mathrm{PGA}=0.20 \mathrm{~g}$ 


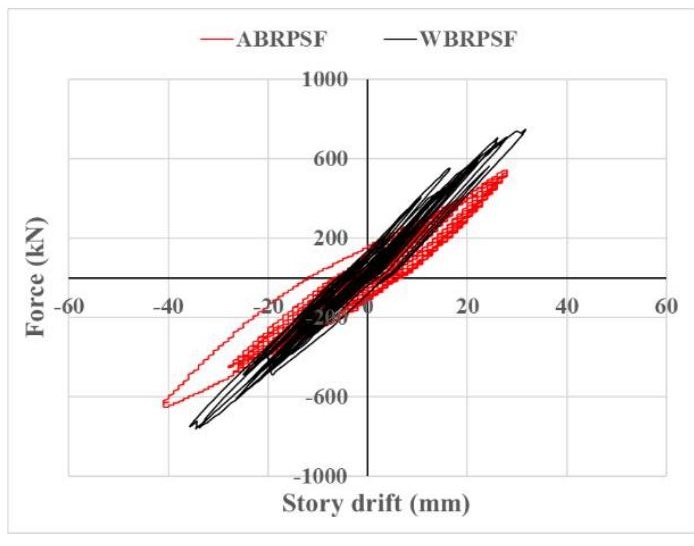

(c) $\mathrm{PGA}=0.40 \mathrm{~g}$

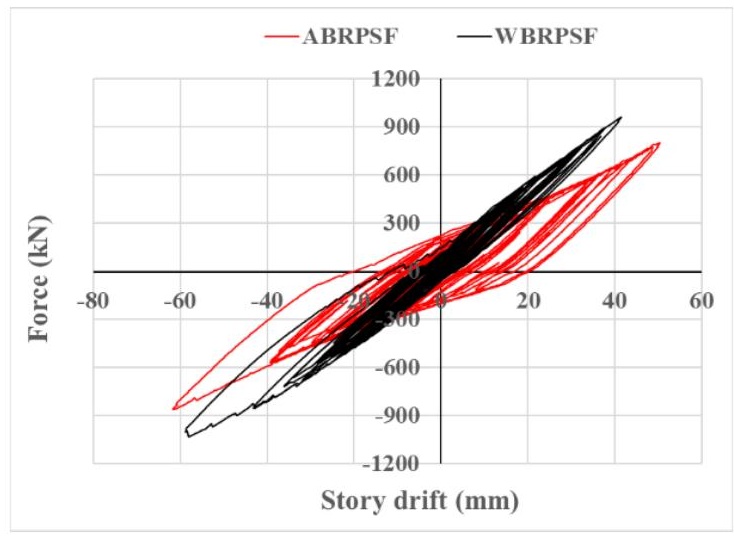

(e) $\mathrm{PGA}=0.62 \mathrm{~g}$

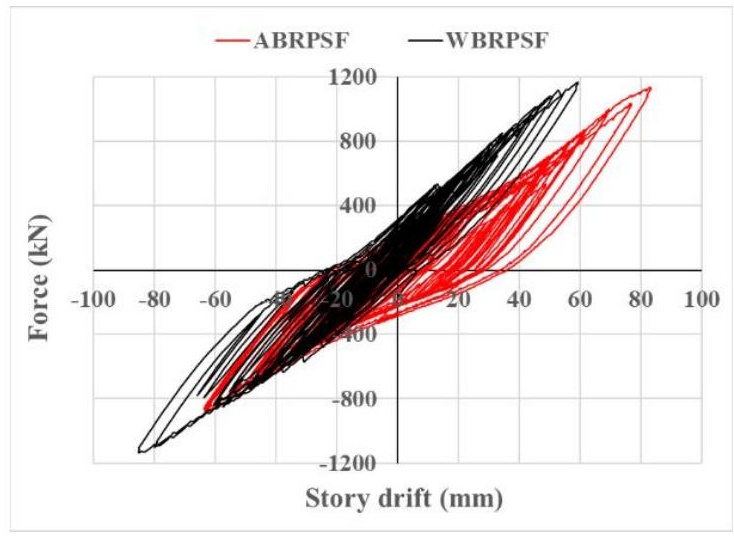

(g) $\mathrm{PGA}=1.0 \mathrm{~g}$

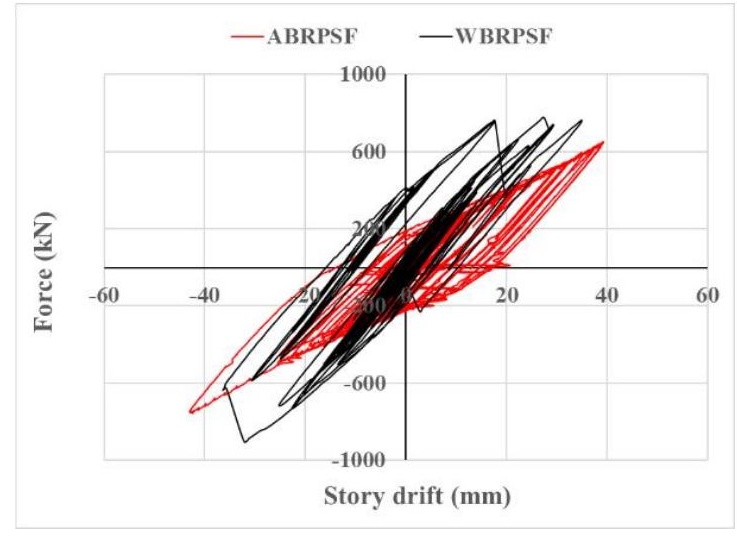

(d) $\mathrm{PGA}=0.51 \mathrm{~g}$

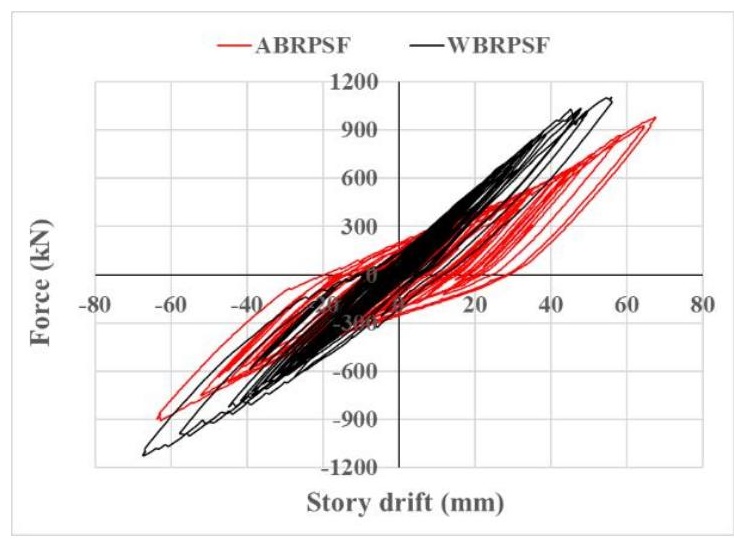

(f) $\mathrm{PGA}=0.81 \mathrm{~g}$

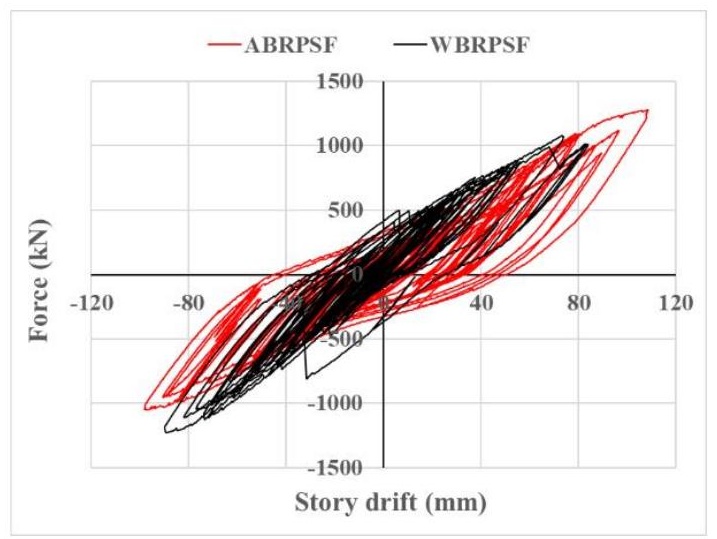

(h) $\mathrm{PGA}=1.2 \mathrm{~g}$

Fig. 18 Hysteretic curves of ABRPSF and WBRPSF under Wenchuan ground motion records

\subsection{Strain development}

Comparative results pertaining to the strain development of the typical regions of the ABRPSF and WBRPSF under fortification and rare seismic actions are shown in Figs. 19 and 20. The yield strain $\varepsilon_{\mathrm{y}}$ adopted in this study was $1800 \mu \varepsilon$, which was obtained based on the completed material test. The strain gauge was used to measure the largest strain for each position. The column foot investigated in this study represented the peak strain value on the column foot flange; column base flange, which is the flange of the column parallel to the altitude of the beam; panel zone, which is the column web plate in the area of the beam-to-column connection; flanges of the short and long beams, which were in the upper flange area closer to the vertical plate; and the upper surface of the reinforced plate of the long beam that was closer to the vertical plate.
The comparative time history curves of strain variation for both steel frames under 0.20 and $0.51 \mathrm{~g}$ of the EL-Centro seismic records are shown in Figs. 19 and 20, respectively. Furthermore, Table 5 lists the detailed data for strain development. As shown in the figures, the strain for both steel frames was at the same level and remained at a relatively low level apart from the reinforced plate. Moreover, the strain developments of the column foot and column base flanges of the WBRPSF was larger than that of the ABRPSF. Meanwhile, the peak strain appeared at the reinforced plate of the ABRPSF, which reached $1132.7 \mu \varepsilon$ and was still below the yield level. When PGA $=0.51 \mathrm{~g}$, the maximum story drifts of the ABRPSF and WBRPSF were 1/110 and 1/126 rad, which were less than the elastic story drift limit of 1/50 rad according to the Code for Seismic Design of Buildings . Furthermore, both steel frames remained at an elastic state upon unloading under the EL-Centro records. 


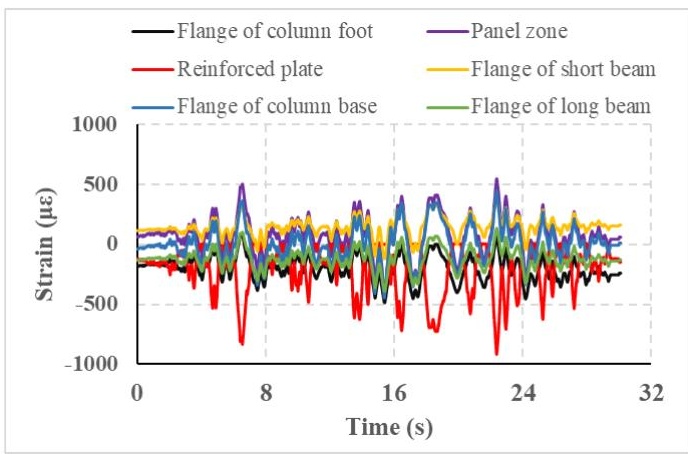

(a) ABRPSF

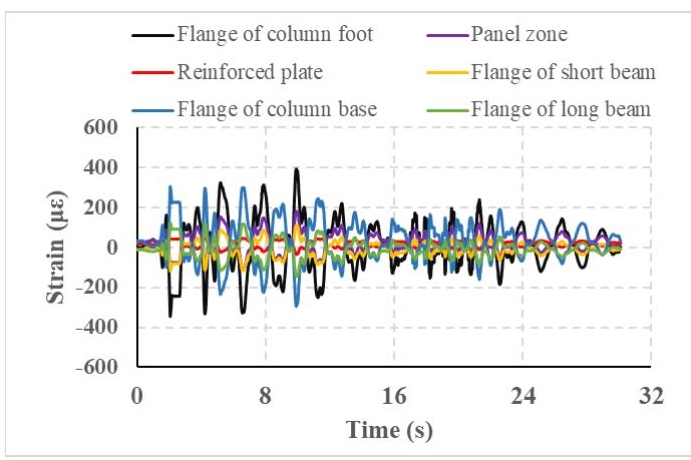

(b) WBRPSF

Fig. 19 Time history curves of strain development under EL-Centro seismic records (PGA $=0.20 \mathrm{~g}$ )

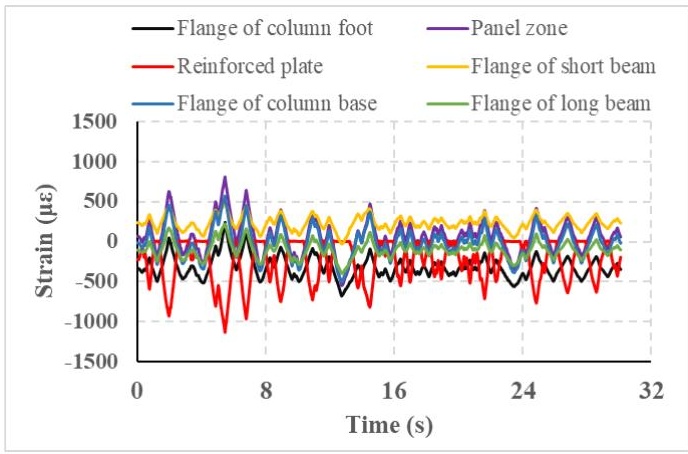

(a) ABRPSF

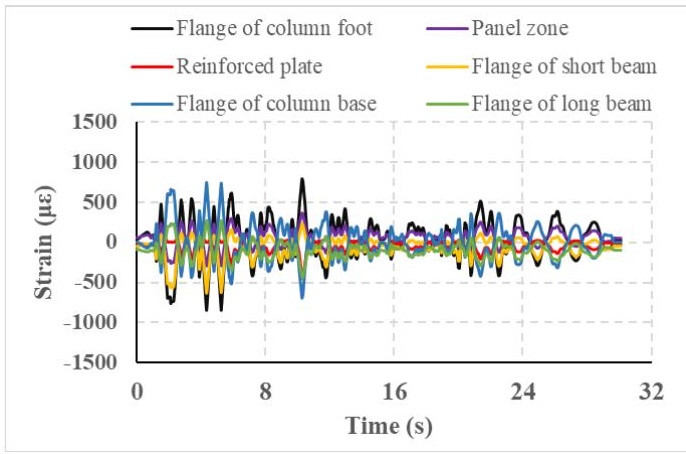

(b) WBRPSF

Fig. 20 Time history curves of strain development under EL-Centro seismic records (PGA $=0.51 \mathrm{~g}$ )

Table 5

Strain development at typical regions for both steel frames under EL-Centro seismic records

\begin{tabular}{|c|c|c|c|c|c|c|c|c|}
\hline Steel frames & PGA & Flange of column foot & Panel zone & Reinforced plate & Flange of short beam & Flange of column base & Big L plate & Small L plate \\
\hline \multirow{4}{*}{ ABRPSF } & $0.07 \mathrm{~g}$ & 211.1 & 155.4 & 4.8 & 115.7 & 111.8 & 131.3 & 68.4 \\
\hline & $0.20 \mathrm{~g}$ & 390.4 & 435.3 & 776.7 & 318.5 & 328.8 & 246.9 & 284.8 \\
\hline & $0.40 \mathrm{~g}$ & 580.3 & 700 & 1031.3 & 451 & 586.2 & 484.9 & 553.4 \\
\hline & $0.51 \mathrm{~g}$ & 678.3 & 808.7 & 1132.7 & 495.9 & 571.8 & 630.1 & 721.1 \\
\hline \multirow{4}{*}{ WBRPSF } & $0.07 \mathrm{~g}$ & 373.2 & 93.9 & 30.1 & 64.9 & 187.7 & - & - \\
\hline & $0.20 \mathrm{~g}$ & 440.7 & 199.5 & 38.9 & 170.2 & 813.0 & - & - \\
\hline & $0.40 \mathrm{~g}$ & 815.8 & 304.3 & 195.7 & 352.5 & 353.6 & - & - \\
\hline & $0.51 \mathrm{~g}$ & 847.4 & 368.2 & 446.3 & 632.3 & 746.4 & - & - \\
\hline
\end{tabular}

Figs. 21-26 show the strain development of typical positions of both steel records, and the detailed data are shown in Table 6. frames under $0.20,0.40,0.62,0.81,1.0$, and $1.2 \mathrm{~g}$ of the Wenchuan seismic

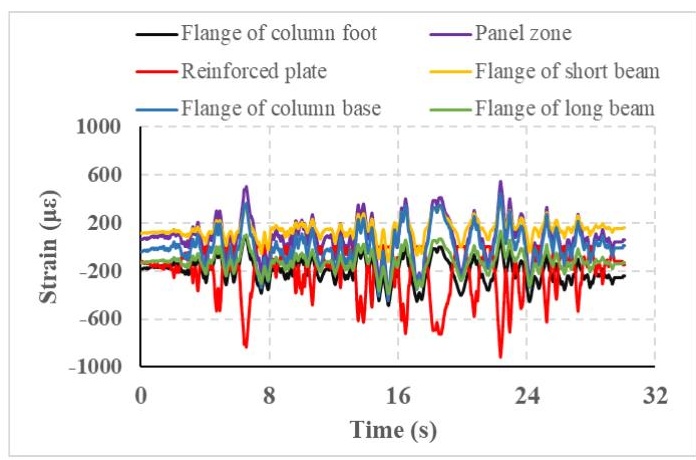

(a) ABRPSF

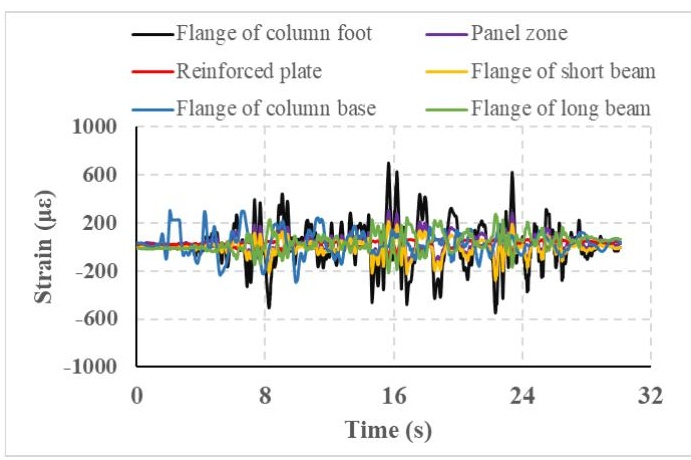

(b) WBRPSF

Fig. 21 Time history curves of strain development under Wenchuan seismic records (PGA $=0.20 \mathrm{~g}$ ) 


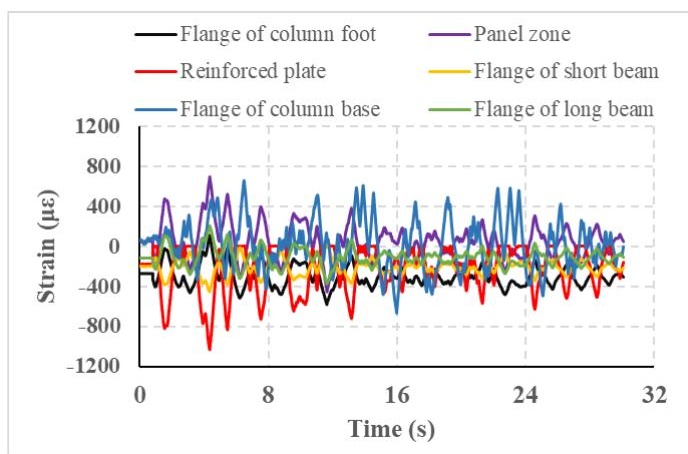

(a) ABRPSF

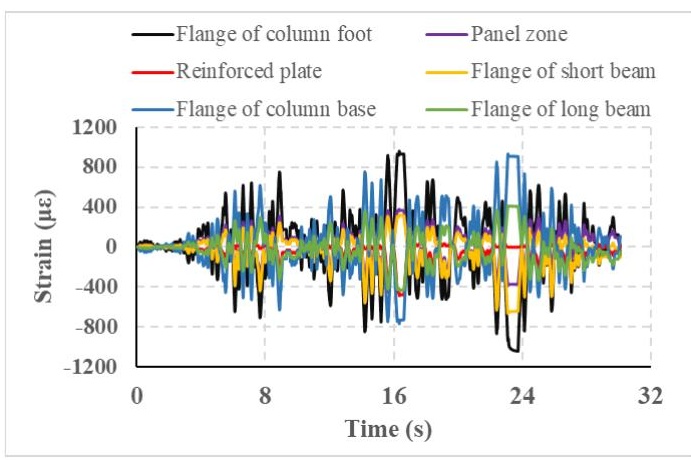

(b) WBRPSF

Fig. 22 Time history curves of strain development under Wenchuan seismic records (PGA $=0.40 \mathrm{~g}$ )

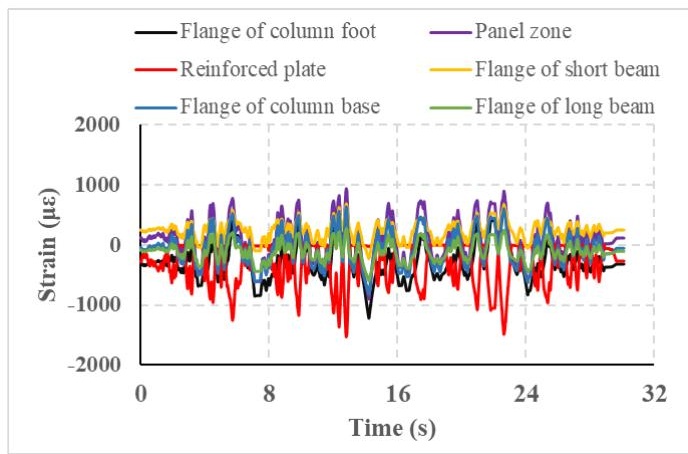

(a) ABRPSF

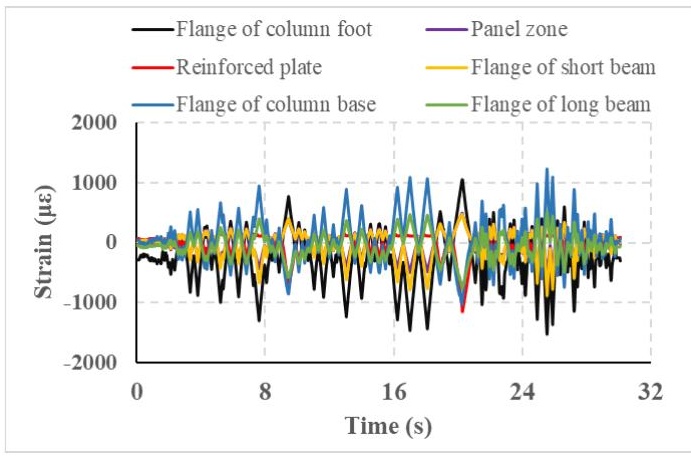

(b) WBRPSF

Fig. 23 Time history curves of strain development under Wenchuan seismic records (PGA $=0.62 \mathrm{~g}$ )

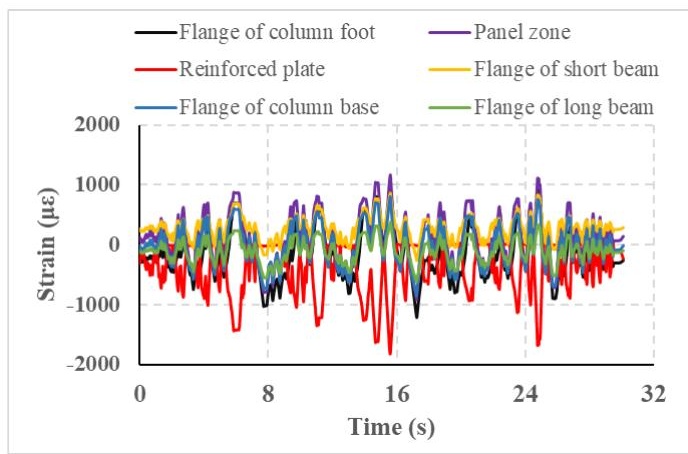

(a) ABRPSF

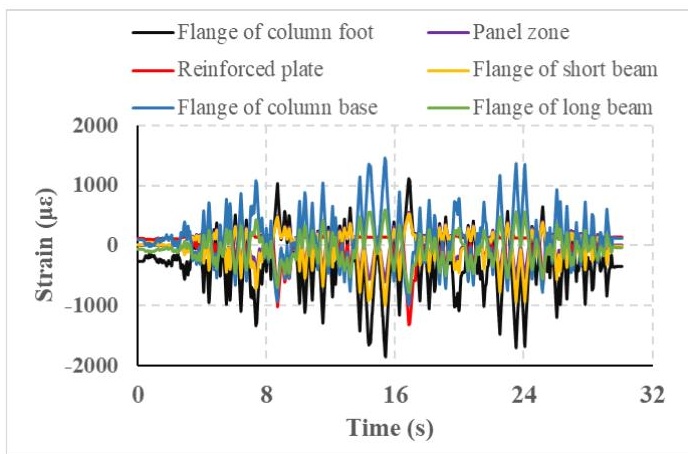

(b) WBRPSF

Fig. 24 Time history curves of strain development under Wenchuan seismic records $(\mathrm{PGA}=0.81 \mathrm{~g})$

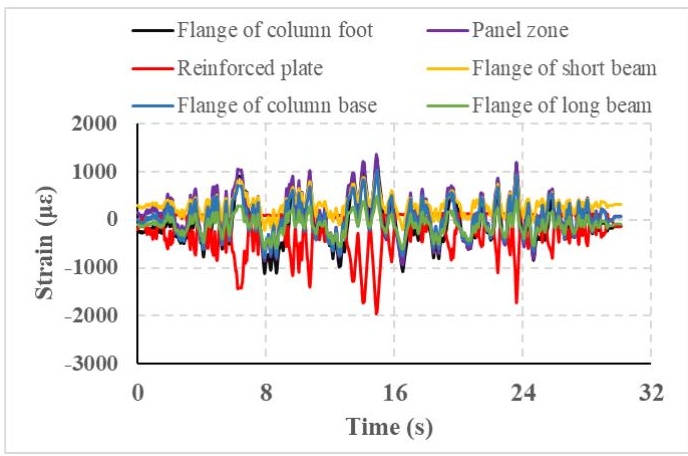

(a) ABRPSF

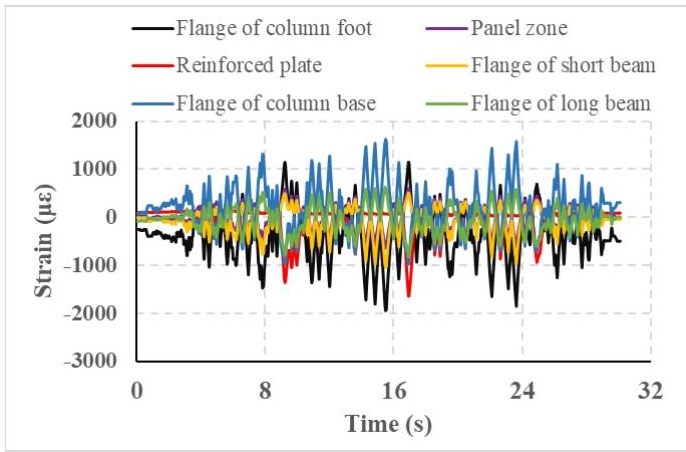

(b) WBRPSF

Fig. 25 Time history curves of strain development under Wenchuan seismic records ( $\mathrm{PGA}=1.0 \mathrm{~g}$ ) 


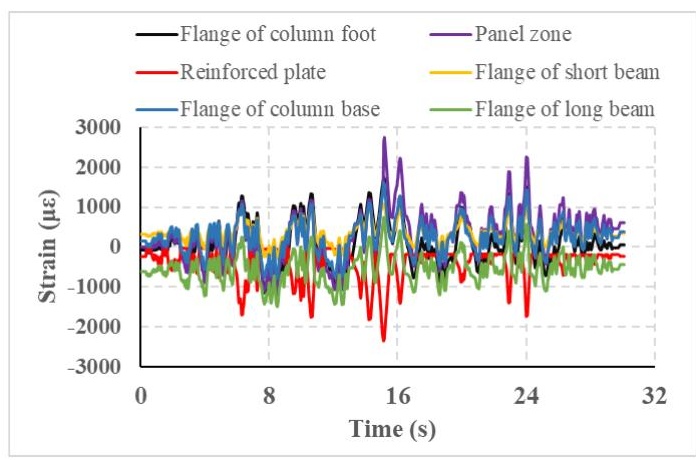

(a) ABRPSF

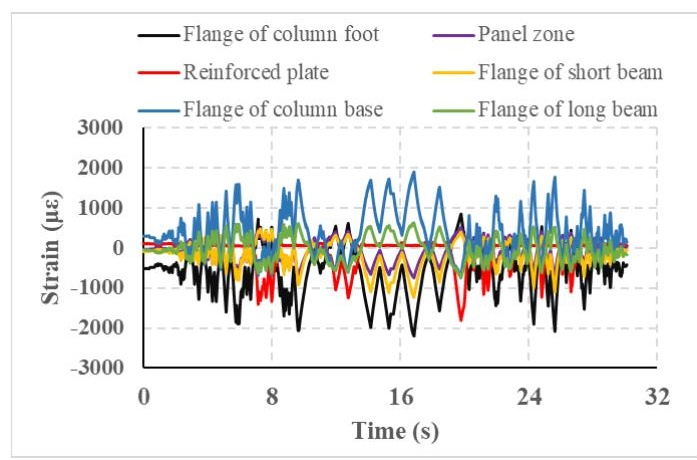

(b) WBRPSF

Fig. 26 Time history curves of strain development under Wenchuan seismic records (PGA $=1.2 \mathrm{~g}$ )

When the PGA reached $0.62 \mathrm{~g}$, the maximum story drifts of the ABRPSF and WBRPSF were $1 / 56$ and $1 / 65 \mathrm{rad}$, respectively, whereas the maximum strains were $1529.5 \mu \varepsilon$ and $1522.9 \mu \varepsilon$, respectively. Both steel frames still remained in an elastic state and exhibited good recentering capacities. When PGA $=0.81 \mathrm{~g}$, the maximum story drifts of the ABRPSF and WBRPSF were $1 / 47$ and $1 / 56 \mathrm{rad}$, respectively; plasticity started to develop for both steel frames, and they were still in the same level. The column foot flange of the WBRPSF entered the plastic state but the column foot flange of the ABRPSF remained elastic. When $\mathrm{PGA}=1.2 \mathrm{~g}$, the maximum story drifts of the ABRPSF and WBRPSF were $1 / 29$ and $1 / 34 \mathrm{rad}$, respectively, exceeding the elastic story drift limit of 1/50 rad according to the Code for Seismic Design of Buildings Meanwhile, the peak strains of the ABRPSF and WBRPSF reached 2746.2 $\mu \varepsilon$ and $2198.8 \mu \varepsilon$, respectively, and the column foot flange of the ABRPSF was still in the elastic state. Both frames can still return to their original positions after an earthquake and indicated have good resilient capacities and excellent seismic behaviors.

Table 6

Strain development at typical regions for both steel frames under Wenchuan seismic records

\begin{tabular}{|c|c|c|c|c|c|c|c|c|}
\hline Steel frames & PGA & Flange of column foot & Panel zone & Reinforced plate & Flange of short beam & Flange of column base & Big L-plate & Small L-plate \\
\hline \multirow{8}{*}{ ABRPSF } & $0.07 \mathrm{~g}$ & 271.3 & 202.3 & 87.68 & 126.94 & 201.96 & 208.6 & 119.5 \\
\hline & $0.20 \mathrm{~g}$ & 487.0 & 548.0 & 918.0 & 377.0 & 448.0 & 387.0 & 313.0 \\
\hline & $0.40 \mathrm{~g}$ & 580.3 & 700.0 & 1031.3 & 451.0 & 666.0 & 707.0 & 671.0 \\
\hline & $0.51 \mathrm{~g}$ & 1090.1 & 809.2 & 1281.0 & 580.4 & 745.5 & 861.5 & 984.6 \\
\hline & $0.62 \mathrm{~g}$ & 1218.4 & 938.0 & 1529.5 & 685.0 & 823.8 & 958.4 & 1039.5 \\
\hline & $0.81 \mathrm{~g}$ & 1214.1 & 1168.8 & 1820.7 & 863.8 & 843.4 & 1021.5 & 1148.1 \\
\hline & $1.0 \mathrm{~g}$ & 1267.8 & 1364.6 & 1960.3 & 974.4 & 1035.3 & 1140.9 & 1119.1 \\
\hline & $1.2 \mathrm{~g}$ & 1746.7 & - & 2349.7 & 1079.9 & 1615.6 & 1392.2 & 1049.3 \\
\hline \multirow{8}{*}{ WBRPSF } & $0.07 \mathrm{~g}$ & 236.5 & 98.6 & 27.2 & 65.1 & 182.2 & - & - \\
\hline & $0.20 \mathrm{~g}$ & 700.1 & 307.5 & 144.0 & 278.2 & 569.9 & - & - \\
\hline & $0.40 \mathrm{~g}$ & 1044.1 & 385.8 & 487.8 & 666.5 & 932.2 & - & - \\
\hline & $0.51 \mathrm{~g}$ & 1136.2 & 472.4 & 764.4 & 732.0 & 1031.1 & - & - \\
\hline & $0.62 \mathrm{~g}$ & 1522.9 & 536.1 & 1148.3 & 882.5 & 1229.3 & - & - \\
\hline & $0.81 \mathrm{~g}$ & 1854.6 & 605.2 & 1320.7 & 995.01 & 1458.6 & - & - \\
\hline & $1.0 \mathrm{~g}$ & 1944.8 & 640.4 & 1643.4 & 1053.6 & 1624.9 & - & - \\
\hline & $1.2 \mathrm{~g}$ & 2198.8 & 745.5 & 1809.4 & 1235.5 & 1904.8 & - & - \\
\hline
\end{tabular}

Here, "_" refers to blank data or data with error.

\subsection{PT force}

Variations of the PT force of steel strand S1 for the ABRPSF and WBRPSF under different levels of EL-Centro seismic records are shown in Figs. 27(a)(d), where the ordinate represents the ratio of the actual PT force to the ultimate PT force. The initial PT force $\mathrm{T}_{0}$ of each steel strand for both steel frames were selected as $0.25 \mathrm{Tu}(0.25 \times 582=145.5 \mathrm{kN}$, where Tu is the ultimate PT force measuring $582 \mathrm{kN}$ ), which can provide adequate self-centering capacity and simultaneously avoid the potential issue of steel strand yielding.

As shown in Fig. 27, the PT force fluctuated around the initial PT force $(0.25 \mathrm{Tu})$ for both steel frames under a PGA of $0.07 \mathrm{~g}$, thereby causing no gap opening. When PGA $=0.20 \mathrm{~g}$, a slight gap opening occurred; consequently, several marked protruding points appeared on the time history curves of the PT force variation. The maximum PT forces of the ABRPSF and WBRPSF reached $0.252 \mathrm{Tu}$ and $0.254 \mathrm{Tu}$, respectively. With the increase in the gap openings, the length of the prestressed strand increased, causing the PT force to increase as well. When the PGA reached 0.40 and $0.51 \mathrm{~g}$, the PT force of both steel frames increased significantly, in which the maximum PT forces of the ABRPSF and WBRPSF when PGA $=0.40 \mathrm{~g}$ were $0.258 \mathrm{Tu}$ and $0.261 \mathrm{Tu}$, respectively, and when PGA $=0.51 \mathrm{~g}$, they were $0.261 \mathrm{Tu}$ and $0.263 \mathrm{Tu}$, respectively. It can be concluded that under various EL-Centro seismic levels, the PT force of the ABRPSF was smaller than that of the WBRPSF, and a slight PT force decrease occurred in both steel frames when the test was terminated, as shown in Table 7. 


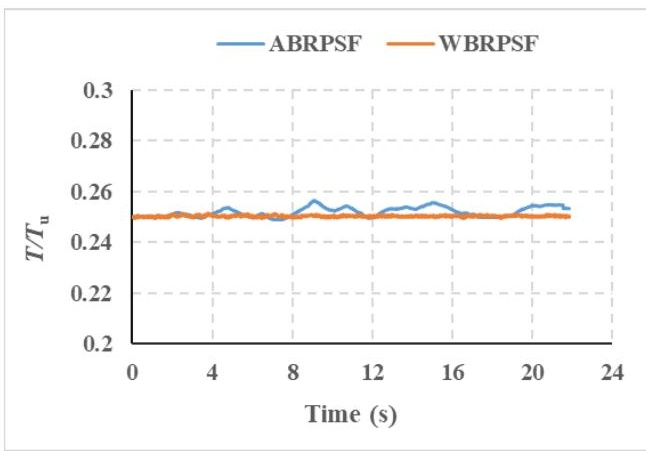

(a) $\mathrm{PGA}=0.07 \mathrm{~g}$

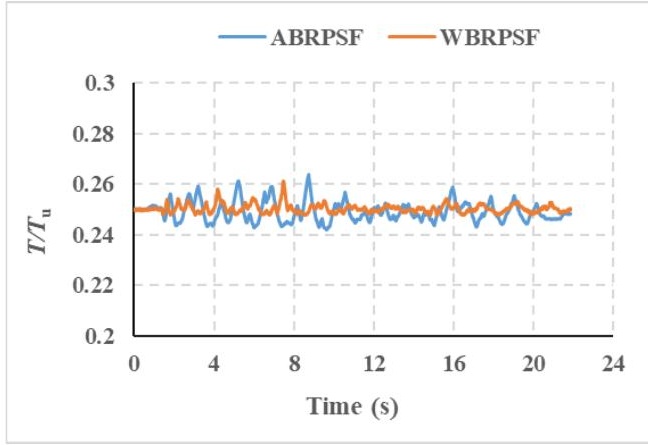

(c) $\mathrm{PGA}=0.40 \mathrm{~g}$

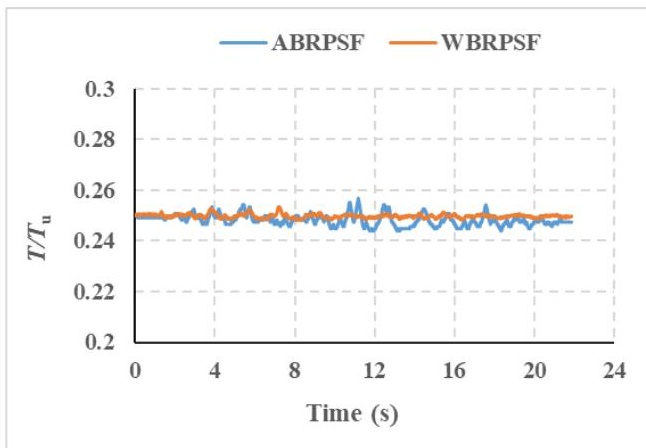

(b) $\mathrm{PGA}=0.20 \mathrm{~g}$

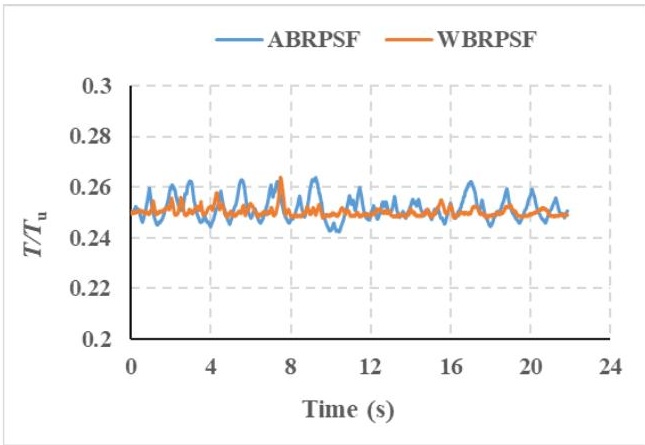

(d) $\mathrm{PGA}=0.51 \mathrm{~g}$

Fig. 27 PT force variations of both steel frames under different EL-Centro seismic levels

The variations in the PT force of the ABRPSF and WBRPSF under the Wenchuan seismic records are shown in Figs. 28(a)-(h). Similar to that under the EL-Centro seismic level, the variation in the PT force still fluctuated around the initial PT force when PGA $=0.20 \mathrm{~g}$. With the gap opening, the variation in the PT force increased significantly. When PGA $=0.40 \mathrm{~g}$, the maximum PT forces of the ABRPSF and WBRPSF were 0.269Tu and 0.281Tu, respectively, and under $0.51 \mathrm{~g}$, they were $0.270 \mathrm{Tu}$ and $0.278 \mathrm{Tu}$, respectively. Meanwhile, the PT forces of the ABRPSF and WBRPSF decreased by $6.46 \%$ and $1.51 \%$, respectively. When PGA $=0.62 \mathrm{~g}$, the maximum PT forces of the ABRPSF and WBRPSF were 0.301Tu and 0.323Tu, respectively; when the PGA reached $0.81 \mathrm{~g}$, the maximum PT forces increased to $0.305 \mathrm{Tu}$ and $0.336 \mathrm{Tu}$, respectively. With the increase in seismic levels, the maximum PT force continued to increase.

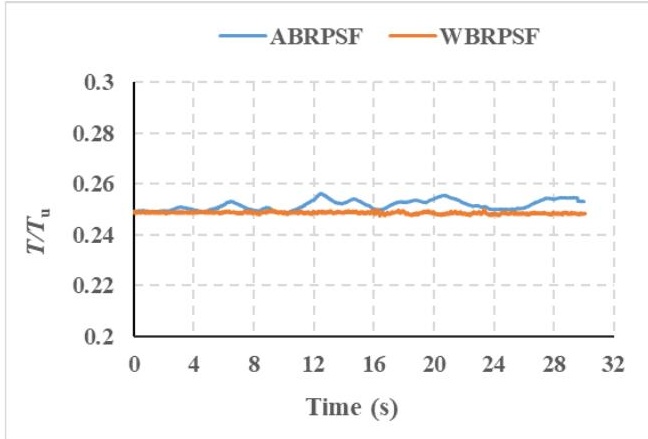

(a) $\mathrm{PGA}=0.07 \mathrm{~g}$

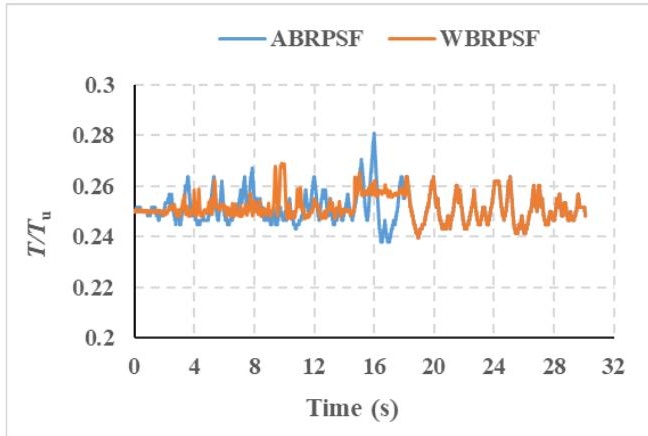

(c) $\mathrm{PGA}=0.40 \mathrm{~g}$
When the PGA increased to $1.0 \mathrm{~g}$, the maximum PT forces of the ABRPSF and WBRPSF reached 0.296Tu and 0.359Tu, respectively; when PGA was $1.2 \mathrm{~g}$, the maximum PT forces were $0.344 \mathrm{Tu}$ and $0.364 \mathrm{Tu}$, respectively. The PT force decrease of the ABRPSF and WBRPSF under the Wenchuan seismic records from 0.07 to $1.2 \mathrm{~g}$ was small, as shown in Table 7 . We observed that the maximum PT force for both steel frames showed similar variation trends, although the maximum PT force of the ABRPSF was relatively smaller than that of the WBRPSF because the L-plates underwent an elastic deformation and bolt slippage occurred on the beam-to-column connection of the ABRPSF; additionally, the slight decrease indicated that the strands, anchorage device performance, and prestressed method were reliable.

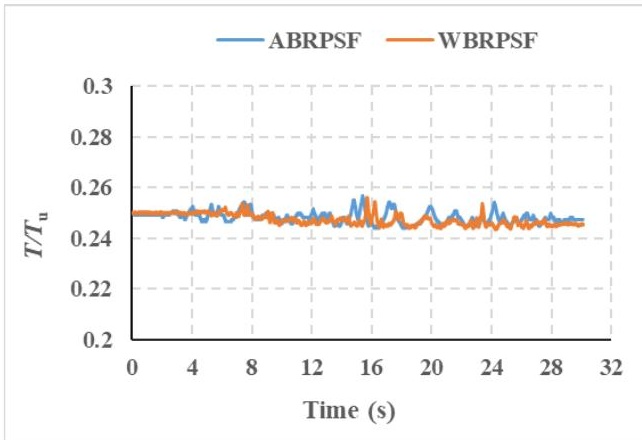

(b) $\mathrm{PGA}=0.20 \mathrm{~g}$

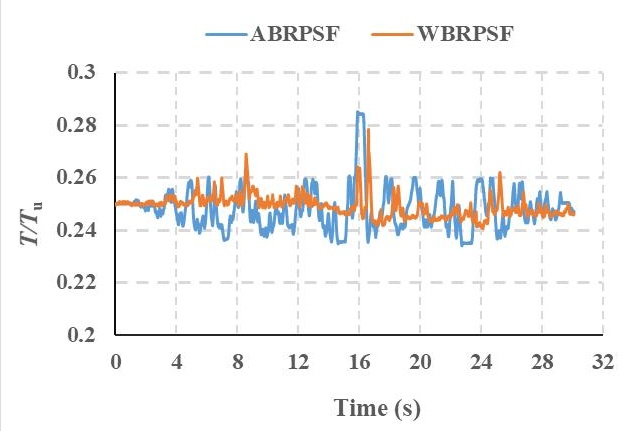

(d) $\mathrm{PGA}=0.51 \mathrm{~g}$ 


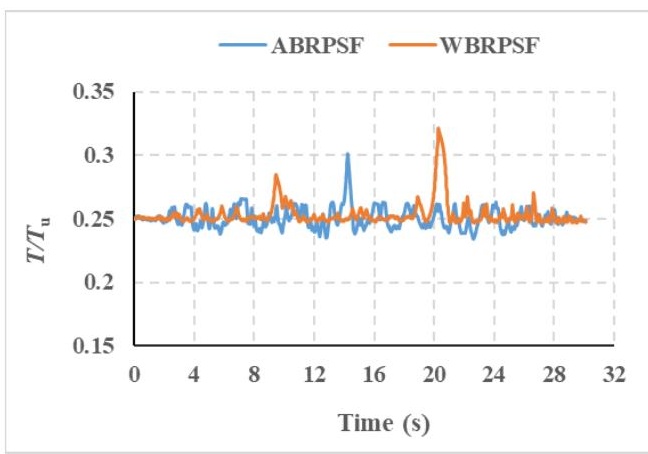

(e) $\mathrm{PGA}=0.62 \mathrm{~g}$

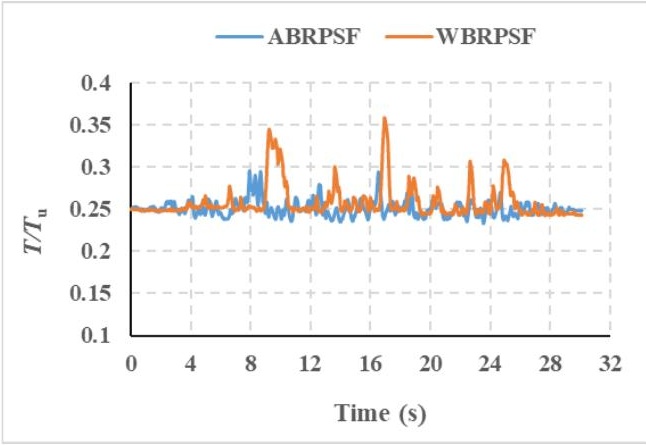

(g) $P G A=1.0 \mathrm{~g}$

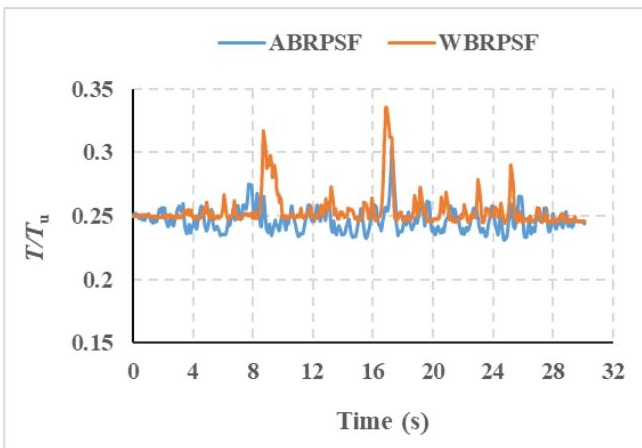

(f) $\mathrm{PGA}=0.81 \mathrm{~g}$

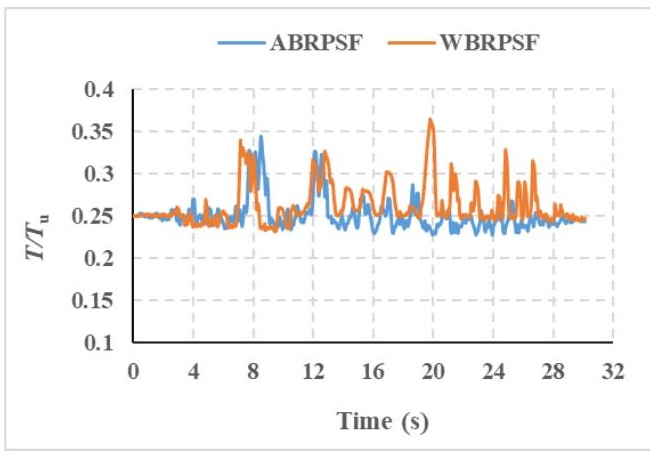

(h) $\mathrm{PGA}=1.2 \mathrm{~g}$

Fig. 28 PT force variations of both steel frames under different Wenchuan seismic levels

Table 7

PT force decrease under various seismic levels

\begin{tabular}{|c|c|c|c|c|c|c|c|}
\hline \multicolumn{2}{|c|}{ Seismic event } & \multicolumn{2}{|c|}{ initial PT force } & \multicolumn{2}{|c|}{ PT force decrease } & \multicolumn{2}{|c|}{ Ratio of PT force decrease } \\
\hline Ground motion & PGA & $T_{0} / \mathrm{kN}$ & $T_{0} / \mathrm{T}_{\mathrm{u}}$ & $\operatorname{ABRPSF}\left(T_{\mathrm{d}} / \mathrm{kN}\right)$ & $\operatorname{WBRPSF}\left(T_{\mathrm{d}} / \mathrm{kN}\right)$ & ABRPSF & WBRPSF \\
\hline \multirow{4}{*}{ EL-Centro } & $0.07 \mathrm{~g}$ & 145.5 & 0.25 & 0.000 & 0.873 & $0.000 \%$ & $0.600 \%$ \\
\hline & $0.20 \mathrm{~g}$ & 145.5 & 0.25 & 0.287 & 2.172 & $0.197 \%$ & $1.493 \%$ \\
\hline & $0.40 \mathrm{~g}$ & 145.5 & 0.25 & 5.138 & 0.602 & $3.531 \%$ & $0.414 \%$ \\
\hline & $0.51 \mathrm{~g}$ & 145.5 & 0.25 & 0.562 & 0.863 & $0.386 \%$ & $0.593 \%$ \\
\hline \multirow{8}{*}{ Wenchuan } & $0.07 \mathrm{~g}$ & 145.5 & 0.25 & 0.000 & 1.050 & $0.000 \%$ & $0.722 \%$ \\
\hline & $0.20 \mathrm{~g}$ & 145.5 & 0.25 & 1.500 & 2.740 & $1.031 \%$ & $1.883 \%$ \\
\hline & $0.40 \mathrm{~g}$ & 145.5 & 0.25 & 1.000 & 1.000 & $0.687 \%$ & $0.687 \%$ \\
\hline & $0.51 \mathrm{~g}$ & 145.5 & 0.25 & 9.400 & 2.200 & $6.460 \%$ & $1.512 \%$ \\
\hline & $0.62 \mathrm{~g}$ & 145.5 & 0.25 & 0.800 & 1.240 & $0.550 \%$ & $0.852 \%$ \\
\hline & $0.81 \mathrm{~g}$ & 145.5 & 0.25 & 3.500 & 2.716 & 2.406 & 1.867 \\
\hline & $1.00 \mathrm{~g}$ & 145.5 & 0.25 & 1.000 & 4.250 & 0.687 & 2.921 \\
\hline & $1.20 \mathrm{~g}$ & 145.5 & 0.25 & 3.900 & 1.780 & 2.680 & 1.223 \\
\hline
\end{tabular}

\section{Summary and Conclusions}

An innovative ABRPSF was proposed and experimentally investigated by comparing it with a WBRPSF. Based on the comparison of pseudo-dynamic test results under various seismic levels, i.e., $\mathrm{PGA}=0.07,0.20,0.40,0.51,0.62$, $0.81,1.0$, and $1.2 \mathrm{~g}$ for both structural systems, the following conclusions were obtained:

(1) When PGA $=0.07 \mathrm{~g}$, the maximum story drifts of the ABRPSF and WBRPSF were $1 / 518$ and $1 / 529 \mathrm{rad}$, respectively, under the Wenchuan seismic records; those values did not exceed the elastic story drift limit $(1 / 250 \mathrm{rad})$ defined in the Code for Seismic Design of Buildings [28]. Furthermore, the maximum story drift of the ABRPSF was greater than that of the WBRPSF, indicating that the rigidity of the ABRPSF was slightly smaller than that of the WBRPSF. No gap opening or damage occurred in both steel frames under frequent earthquakes. The hysteretic curves of both steel frames presented a linear shape and both steel frames remained in the elastic state. The maximum PT forces of the steel strands of both steel frames were close to the initial value without any obvious decrease after a test was performed. The performances of both steel frames satisfied the design goal of "no gap opening and no damage occurs during frequent earthquakes."

(2) When the seismic level reached that of an 8-degree fortification earthquake with a PGA of $0.2 \mathrm{~g}$, the maximum story drifts of both steel frames under the Wenchuan seismic records were nearly identical, i.e., 1/173 and 1/171 rad for the ABRPSF and WBRPSF, respectively. The gap opening of the WBRPSF was slightly larger that of the ABRPSF, and the values for both remained at relatively low. Additionally, almost no residual gap opening was observed when loading completed, indicating that both steel frames possessed favorable recentering capacities. Both steel frames began to dissipate energy at this time, as observed from slight loops that formed in the hysteretic curves. Both steel frames remained in the elastic state during loading. The maximum PT force of the ABRPSF steel strands was less than that of the WBRPSF, and the PT forces of both steel frames almost did not decrease after the test. The performances of both steel frames satisfied the design goal of "gap openings are formed to dissipate energy and no damage occurs to the main structure under fortification earthquake action."

(3) When the PGA varied from 0.4 to $0.62 \mathrm{~g}$, the test results of the ABRPSF and WBRPSF presented similar trends. When PGA $=0.62 \mathrm{~g}$, the maximum story drift of the ABRPSF (1/56 rad) was greater than that of the WBRPSF $(1 / 65 \mathrm{rad})$, 
and both values did not exceed the elastoplastic story drift limit (1/50 rad) [28]. The gap-opening response of the ABRPSF was smaller than that of the WBRPSF owing to the bolt slippage and the elastic deformation of the L-plate for the connection of the ABRPSF. Furthermore, no residual gap openings were observed for both steel frames; both frames were able to return to their original positions upon rare or even extremely rare earthquakes. Additionally, the ABRPSF had a more satisfactory energy dissipating capacity than the WBRPSF owing to the dual energy dissipating mechanisms of bolt slippage on the beamto-column connection and the web friction device of the ABRPSF. Both steel frames remained in the elastic state under a 9-degree rare earthquake. The maximum PT force of the ABRPSF was still less than that of the WBRPSF, and the values of both did not exceed the yield strength of steel strands. After the test, the PT forces of both steel frames were still similar to the initial value, and both structures still possessed adequate re-centering and bearing capacities to endure intensive aftershocks upon rare earthquakes. The performance of both steel frames satisfied the design goal of "only small structural damage occurs and the structure can still operate normally during rare or extremely rare earthquakes."

(4) When the PGA increased to $0.81,1.0$, and $1.2 \mathrm{~g}$, the results of the ABRPSF and WBRPSF were similar. For example, when PGA $=1.2 \mathrm{~g}$, the maximum story drifts for the ABRPSF and WBRPSF were $1 / 29$ and 1/34 rad, respectively, and the gap openings of both steel frames developed further and exhibited the same trend as that of PGA $=0.62 \mathrm{~g}$. The residual gap openings of both steel frames were approximately zero, indicating that both steel frames can

\section{References}

[1] Ricles, J.M., Sause, R., Garlock, M.M. and Zhao. C., "Post-tensioned seis-mic-resistant connections for steel frames", Journal of Structural Engineering, 127(2), 113-121, 2001.

[2] Ricles, J.M., Sause, R., Peng, S. and Lu, L., "Experimental evaluation of earthquake resistant post-tensioned steel connections", Journal of Structural Engineering, 128(7), 850-859, 2002.

[3] Christopoulos, C., Filiatrault, A., Uang, C.M. and Folz, B., "Post-tensioned Energy Dissipating Connections for Moment-resisting Steel Frames", Journal of Structural Engineering, 128(9), 1111-1120, 2002.

[4] Tsai, K. C., Chou, C. C., Lin, C. L., Chen, P. C. and Jhang, S. J., "Seismic Self-centering Steel Beam-to- column Moment Connections using Bolted Friction Devices, "Earthquake Engineering and Structural Dynamics, 37, 627-645, 2008.

[5] Iyama, J., Seo, C.Y., Ricles, J.M. and Sause, R., "Self-centering MRFs with Bottom Flange Friction Devices under Earthquake Loading", Journal of Constructional Steel Research, 65, 314-325, 2009.

[6] Wolski, M., Ricles, J.M. and Sause, R., "Experimental Study of a Self-centering Beamcolumn Connection with Bottom Flange Friction Device", Journal of Structural Engineering, 135(5), 479-488, 2009

[7] Khoo, H.H., Clifton, C., Butterworth, J., MacRae, G., Gledhill, S. and Sidwell, G., "Development of the self-centering Sliding Hinge Joint with friction ring springs", Journal of Constructional Steel Research, 78, 201-211, 2012.

[8] Maria M. Garlock, James M. Ricles, Richard Sause. Experimental Studies of Full-Scale Posttensioned Steel Connections, 131(3), 438-448, 2005.

[9] Ying-Cheng Lin, Richard Sause, James Ricles. Seismic Performance of a Large-Scale Steel Self-Centering Moment-Resisting Frame: MCE Hybrid Simulations and Quasi-Static Pushover Tests, 139(7), 1227-1236, 2013.

[10] Ying-Cheng Lin, Richard Sause, James M. Ricles. Seismic Performance of Steel SelfCentering, Moment-Resisting Frame: Hybrid Simulations under Design Basis Earthquake, 139(11), 1823-1832, 2013

[11] Apostolakis, G., Dargush, G.F. and Filiatrault, A., "Computational Framework for Automated Seismic Design of Steel Frames with Self-Centering Connections", Journal of Computing in Civil Engineering, 28(2), 170-181, 2014

[12] George Vasdravellis, Theodore L. Karavasilis, Brian Uy. Finite element models and cyclic behavior of self-centering steel post-tensioned connections with web hourglass pins, Engineering Structures, 52, 2013.

[13] Xu, X, Zhang, Y.F., Luo, Y.Z, "Self-centering modularized link beams with post-tensioned shape memory alloy rods", Engineering Structures 112, 47-59, 2016.

[14] Sultana, P., Youssef, M.A. "Seismic performance of steel moment resisting frames utilizing superelastic shape memory alloys", Journal of Constructional Steel Research, 125, 239-251, 2016

[15] Farmani, M.A. and Ghassemieh, M. "Steel beam-to-column connections equipped with SMA tendons and energy dissipating devices including shear tabs or web hourglass pins", Journal of Constructional Steel Research, 135, 30-48,2017.

[16] Yam, F.C., M., C.H., Lam, A.C.C. Xie, L.K., "Cyclic performance of extended end-plate connections equipped with shape memory alloy bolts", Journal of Constructional Steel Research, 94, 122-136, 2014

[17] Wei Wang, Cheng Fang, Jia Liu. Self-Centering Beam-to-Column Connections with Combined Superelastic SMA Bolts and Steel Angles, Journal of Structural Engineering, 2016.

[18] Miller, D.J., Fahnestock, L.A., Eatherton, M.R., "Development and experimental validation of a nickel-titanium shape memory alloy self-centering buckling-restrained brace", Engineering Structures, 40, 288-298, 2012.

[19] Qiu, C.X., Zhu, S.Y., "Performance-based seismic design of self-centering steel frames with SMA-based braces", Engineering Structures, 130, 67-82, 2017

[20] Dezfuli, M.A., Dolatshahi, K.M., Mofid, M., Eshkevari, S.S., "Coreless self-centering braces as retrofitting devices in steel structures", Journal of Constructional Steel Research, 133, 485-498, 2017.

[21] Zhang, Y.X., Li, Z.X., Liu, A.R., Li, R. and Liu, X.C., "Research on the behavior of selfcentering replaceable mild steel energy-dissipating braces", ENGINEERING MECHANICS, 34(8), 180-193. 2017

[22] Zhang, Y.X., Li, Z.X., Zhao, W.Z., Li, R., and Li, J.R., "A Performance Study of Beam Column Connections of Self-centering Steel Frame with U-shaped Steel Dampers", Advanced Steel Construction, 12(4), 446-465, 2016

[23] Zhang, A.L., Zhang, Y.X., Li, R. and Wang, Z. Y., "Cyclic Behavior of a Prefabricated Self- still return to their original positions upon extremely rare earthquakes. The new ABRPSF demonstrated more satisfactory energy dissipation performances than the WBRPSF, as observed from the hysteretic curves. As the PGA increased to $0.81 \mathrm{~g}$, the column foot flange of the WBRPSF entered the plastic state; however, the column foot flange of the ABRPSF still remained in the elastic state until PGA $=1.2 \mathrm{~g}$, indicating that the ABRPSF performed better in avoiding plastic damage under rare earthquakes. Under various seismic levels, the PT forces of both steel frames almost did not decrease, indicating that the strands, anchorage device performance, and prestressed method were reliable. Both steel frames demonstrated favorable resilient capacities and satisfying seismic behaviors, and the new ABRPSF can be regarded as a reliable alternative to the WBRPSF

\section{Acknowledgements}

This study is supported by the National Natural Science Foundation of China under Grant No. 51778036, Beijing Municipal Natural Science Foundation under Grant No. 19JH0021, Program for Changjiang Scholars and Innovative Research Team in University under Grant No. IRT_17R06, the Fundamental Research Funds for Beijing Universities under Grant Nos. X19024, X19034, and X19035, Beijing Advanced Innovation Center for Future Urban Design, Beijing University of Civil Engineering and Architecture (Research on Application Technology of High-efficiency Assembly Steel Structure System in Medical Building UDC2019033124), and Science and Technology Program of the Ministry of Housing and Urban-Rural Development (2017-K9-033)

centering Beam-column Connection with a Bolted Web Friction Device", Engineering Structures, 111, 185-198, 2016

[24] Zhang, Y.X., Wang, Z.Y., Zhao, W., Zhao, W.Z., and Chen, Y.Y., " A pseudo-dynamic test study on a self-centering prefabricated steel frame with a column base connected by semirigid joints", Advanced Steel Construction, 12(3), 296-315, 2016.

[25] Zhang, Y.X, Liu, A.R., Zhang, A.L. and Liu, X.C., "Seismic performance analysis of a resilient prestressed steel frame with intermediate column containing friction dampers", Advanced Steel Construction, 13(3), 241-257, 2017.

[26] Zhang, Y.-X., Li, Q.-G., Huang, W.-Z., Jiang, K., Sun, Y.. Behavior of prefabricated beamcolumn connection with short strands in self-centering steel frame. Advanced Steel Construction. 15(2), 203-214, 2019.

[27] GB50019-2017, Standards for design of steel buildings. Beijing: China Architecture \& Building Press; 2016. [in Chinese].

[28] GB50011-2016, Code for seismic design of buildings. Beijing: China Architecture \& Building Press; 2016. [in Chinese]. 


\title{
INFLUENCE OF DIFFERENT SHAPES OF GEOMETRIC IMPERFECTIONS ON THE STRUCTURAL BEHAVIOR OF BEAMS WITH LARGE WEB OPENINGS
}

\author{
Teixeira F. B. *, Caldas R. B. and Grilo L. F. \\ Federal University of Minas Gerais, Belo Horizonte, Brazil \\ * (Corresponding author: E-mail: felipebarbosateixeira@gmail.com)
}

\section{A B S T RA C T}

This paper explores the influence of four different shapes of geometric imperfection upon the collapse of castellated and cellular beams. A numerical model developed in ANSYS and calibrated against experimental results was used to investigate the effects of each imperfection shape. The global imperfection (weak-axis bending) did not affect web-post stability. Webpost bending favored the development of plastic yielding, while web-post misalignment and the local buckling mode strongly compromised web-post stability. The choice of imperfection shape was shown to determine the failure mode of the beams, directly affecting the quality of the numerical results and potentially influencing design decisions.

\begin{tabular}{ll} 
A R T I C L E & H I S T O R Y \\
\hline Received: & 8 October 2019 \\
$\begin{array}{l}\text { Revised: } \\
\text { Accepted: }\end{array}$ & 2 June 2020 June 2020
\end{tabular}

\section{K E Y W O R D S}

large web opening; geometric imperfection; numerical analysis; web-post buckling; lateral-torsional buckling

\section{Introduction}

Castellated and cellular beams are structural elements with large web openings, usually built from a plain-webbed I section. The web of the parent section is cut longitudinally (following a pattern that defines the shape of the openings), the upper and lower halves are moved with respect to each other, and both segments are joined again by welding at the web-post (Fig. 1).
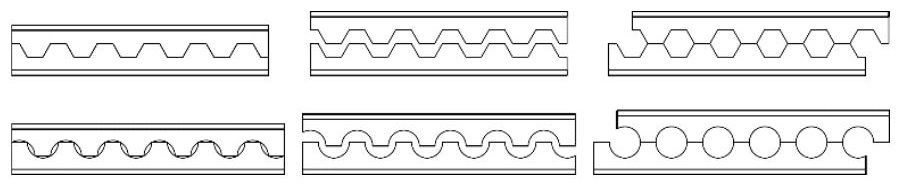

Fig. 1 Conventional process for building castellated beams

Castellated and cellular beams under bending and shear force might display the following failure modes:

- $\quad$ lateral-torsional buckling (LTB, Fig. 2-a) [1,2,3,4];

- $\quad$ plastic hinge, which might occur due to the Vierendeel mechanism (VM, Fig. 2-d) or due to pure bending (PH) [5,6,7];

- $\quad$ longitudinal shear at the web-post (LS, Fig. 2-e) [5];

- $\quad$ web-post buckling due to shear (WBS, Fig. 2-b) [8,9];

- web-post buckling due to compression (WBC, Fig. 2-c) [1,5,10];

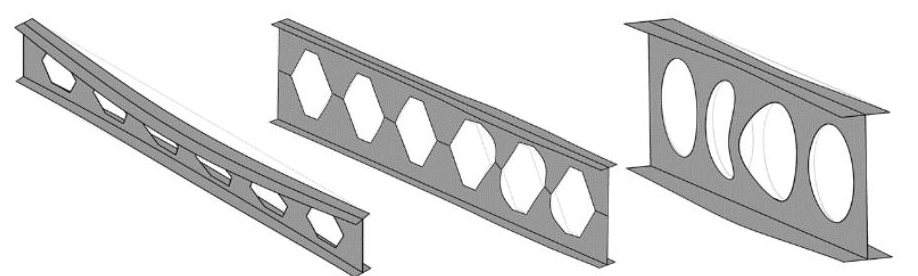

(a)

(b)

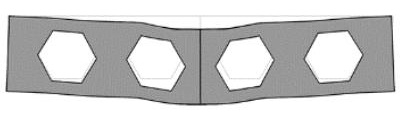

(d)

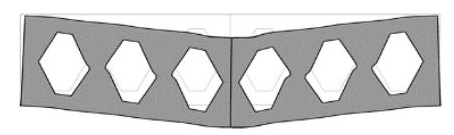

(e)

Fig. 2 Common types of collapse for castellated and cellular beams

Castellated and cellular beams can also be subjected to more improbable failure modes, such as plastic yielding of the web-post due to bending, vertical shearing of the T sections, or the local buckling at the flanges, none of which will be studied in this paper.
The structural behavior of beams with large web openings can be simulated by employing numerical models capable of performing finite element analysis. Such numerical models must be able to accurately predict the mode of failure given the loads, boundary conditions, geometry, and material models. For the study of structural instability in a non-linear analysis the numerical model must be provided with imperfections that are capable of triggering the instability. The standard procedure consists of first performing an eigenvalue analysis to obtain the buckling modes of the beam and then importing the mode shape (eigenvector) associated with the lowest buckling load (eigenvalue). The imported geometry is then used to perform a non-linear analysis $[8,9]$.

The consequences of the shape and magnitude of the geometric imperfection upon the failure mode have been investigated for structures known to be sensitive to instability such as domes [11,12], but not yet for the webs of beams with large web openings. Masri \& Lui [13] showed how the magnitude of the geometric imperfection is directly linked to the flexural resistance of steel delta girders (plain-webbed I beams with inclined plates connecting the compressed flange to the web) to lateral-torsional buckling.

The use of the eigenmode characterized as lateral distortional buckling was adopted by Ellobody [14,15] to successfully study structural failure due to the interaction of combined buckling modes in castellated and cellular beams.

However, the actual initial geometry of the beam is largely a result of the fabrication process, and might not display the shape of the buckling mode [2]. In fact, Nseir et al. [4] and Vieira [8] have measured castellated and cellular beams and shown that the geometric imperfections do not conform to the mode shapes resulting from an eigenvalue analysis. For thin-walled sections Gehring \& Saal [16] have shown how accounting for the actual imperfections that result from the production process affect both the ultimate loads and the shape of the failure mode.

Furthermore, when analyzing beams that, because of their geometry or boundary conditions, lie outside narrow ranges of slenderness, the first buckling mode shape ceases to be the expected one (i.e., web-post buckling due to shear for sturdy beams, and lateral-torsional buckling for slender ones). It is possible to search higher modes for the desired shape, but these usually display a mixture of many local buckling effects, and introducing such an unpredictable shape might compromise the quality of the numerical simulation.

In this paper a novel approach to the introduction of geometric imperfections was studied. The shapes of the geometric imperfections introduced were chosen based on experimental measurements (performed by Vieira [8], Nseir et al. [4], and Sonck [2]) and on factory recommendations by ArcelorMittal [17], a company that manufactures beams with large web openings of both conventional and novel shapes. This approach contrasts with the conventional one, according to which the shape of the imperfection is always the buckling mode and only the magnitude of the imperfection is informed by experimental measurement [8].

\section{Shapes of the geometric imperfections}


The most significant geometric imperfections observed in castellated and cellular beams are:

- global bending of the longitudinal axis of the beam about the weak-axis of inertia (Fig. 3-a);

- $\quad$ bending of the web (Fig. 3-b);

- misalignment between the upper and lower halves of the beam at the welded section (Fig. 3-c).

The shape of the buckling mode (web-post buckling due to shear, Fig. 2-b) was included in this study for the sake of comparison, given that it is adopted in the conventional approach.

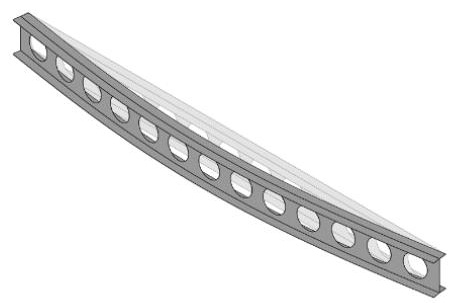

(a)

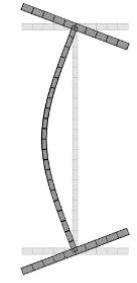

(b)

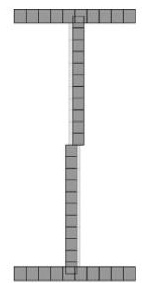

(c)
Fig. 3 Imperfections: a) weak-axis bending; b) web bending; c) web-post misalignment

The weak-axis bending and the web bending shapes were obtained by performing a linear elastic analysis in which a uniformly distributed load was applied (lateral loads at both flanges for the global bending and at mid-depth for the web bending). The buckling modes were obtained by performing an eigenvalue analysis. The web-post misalignment was introduced directly by offsetting the upper and lower halves of the web's cross section in opposite directions.

\section{Finite element modeling and validation}

The finite element package ANSYS 14.0 [18] was used to perform the numerical simulations presented in this paper. In order to study a large quantity of beams, a parametric model, capable of generating any geometry of castellated and cellular beam, was developed using ANSYS's native programming language named APDL (Ansys Parametric Design Language).

\subsection{Experimental result database}

The geometric imperfection numerical study was performed using beams that were subjected to actual physical experiments. Such experiments were collected from the available literature on castellated and cellular beams and were carefully selected in order to include beams that fail globally $[2,4,6]$ and beams that fail locally $[1,6,8,9,19]$. The data collected add up to 42 experiments, each one identified in Table 1 and Table 2, in which: $L$ is the length of the beam; $d_{g}$ is the section depth; $d_{0}$ is the opening height; $b_{f}$ is the flange width; $s_{w}$ is the webpost width; $s_{0}$ is the opening width; $t_{f}$ is the flange thickness; and $t_{w}$ is the web thickness. The experimental setups were either of the three point bending (3P) or four point bending (4P) type, and the load-displacement curves presented throughout this paper follow the template shown in Fig. 4. When displaying results (deformed shapes, strain patterns) the flanges and web-stiffeners of the beams were often omitted, leaving only the web to be shown for greater clarity.
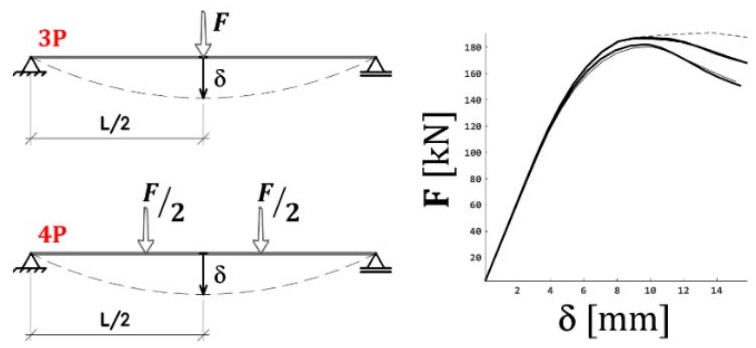

Fig. 4 Diagram of experiments reproduced and load-displacement curve template

Table 1

Castellated beams: geometric data of the experiments compiled (values in $\mathrm{mm}$ )

\begin{tabular}{ccccccccc}
\hline Model & $L$ & $d_{g}$ & $d_{0}$ & $b_{f}$ & $s_{w}$ & $s_{0}$ & $t_{f}$ & $t_{w}$ \\
\hline \multicolumn{8}{c}{ Sonck [2], 4P } \\
CS1_L3 & 3150 & 220 & 118.9 & 83.1 & 73.8 & 136.2 & 7.3 & 5.5 \\
\hline
\end{tabular}

\begin{tabular}{ccccccccc}
\hline CS1_L4 & 3990 & 220 & 118.9 & 83.1 & 73.8 & 136.2 & 7.3 & 5.5 \\
CS1_L6 & 6090 & 220 & 118.9 & 83.1 & 73.8 & 136.2 & 7.3 & 5.5 \\
& & & \multicolumn{7}{c}{ Vieira $[8], 3 \mathrm{P}$} & & & & \\
A1 & 2278 & 452.2 & 301 & 103.7 & 177.2 & 347.3 & 6.1 & 4.9 \\
A2 & 2275 & 450.9 & 298 & 101.8 & 176.5 & 348.3 & 6.1 & 5.1 \\
B1 & 2037 & 453.7 & 301 & 103.5 & 79.9 & 247.2 & 7.1 & 5.7 \\
B2 & 2033 & 451.1 & 302 & 101.4 & 76.8 & 250.6 & 6.1 & 5.1 \\
B3 & 2078 & 458.3 & 307 & 100.7 & 78.6 & 255.0 & 9.1 & 6.6 \\
B4 & 2079 & 462.0 & 310 & 101.7 & 79.8 & 254.2 & 9.3 & 6.4 \\
B5 & 2136 & 472.6 & 316 & 170.5 & 82.7 & 259.5 & 13.1 & 7.8 \\
B6 & 2135 & 472.9 & 317 & 171.3 & 82.3 & 259.9 & 12.5 & 8.0 \\
& & & Veríssimo et al. $[19], 3 \mathrm{P}$ & & & \\
SA1/SA2 & 2251.7 & 450.0 & 300 & 125 & 173.2 & 346.4 & 6.3 & 4.75 \\
SB1/SB2 & 2019.4 & 450.0 & 300 & 125 & 75.4 & 248.6 & 6.3 & 4.75 \\
SB3/SB4 & 2120.4 & 456.5 & 315 & 125 & 79.2 & 261.0 & 8.0 & 6.3 \\
SB5/SB6 & 2187.7 & 487.5 & 325 & 167 & 81.7 & 269.3 & 12.5 & 8.0 \\
SE1/SE2 & 3029.1 & 675.0 & 450 & 150 & 113.1 & 372.9 & 8.0 & 4.75 \\
SX1/SX2 & 3769.5 & 840.0 & 560 & 170 & 140.7 & 464.1 & 8.0 & 4.75 \\
\hline
\end{tabular}

Table 2

Cellular beams: geometric data of the experiments compiled (values in $\mathrm{mm}$ )

\begin{tabular}{|c|c|c|c|c|c|c|c|}
\hline Model & $L$ & $d_{g}$ & $d_{0}$ & $b_{f}$ & $s_{w}$ & $t_{f}$ & $t_{w}$ \\
\hline \multicolumn{8}{|c|}{ Sonck [2], 4P } \\
\hline$C S 2 \_L 3$ & 3150 & 220 & 142.8 & 83.1 & 67.2 & 7.3 & 5.5 \\
\hline$C S 2 \_L 4$ & 3990 & 220 & 142.8 & 83.1 & 67.2 & 7.3 & 5.5 \\
\hline$C S 2 \_L 6$ & 6090 & 220 & 142.8 & 83.1 & 67.2 & 7.3 & 5.5 \\
\hline \multicolumn{8}{|c|}{ Nseir et al. [4], 4P } \\
\hline HEA_340 & 7500 & 472.1 & 345 & 297.7 & 170 & 16.0 & 10.4 \\
\hline$I P E_{-} 330$ & 11000 & 446.5 & 345 & 161.7 & 50 & 10.8 & 7.9 \\
\hline \multicolumn{8}{|c|}{ Tsavdaridis \& D'Mello [9], 3P } \\
\hline$A l T$ & 1700 & 449.8 & 315 & 152.4 & 94.5 & 10.9 & 7.6 \\
\hline$B 1 T$ & 1700 & 449.8 & 315 & 152.4 & 63.0 & 10.9 & 7.6 \\
\hline \multicolumn{8}{|c|}{ Erdal [1], 3P } \\
\hline$E 240 A$ & 2846 & 355.6 & 251 & 106 & 94 & 13.1 & 8.7 \\
\hline$E 240 C$ & 2846 & 355.6 & 251 & 106 & 194 & 13.1 & 8.7 \\
\hline$E 260$ & 2831 & 394.5 & 286 & 113 & 103 & 14.1 & 9.4 \\
\hline$E 280$ & 2820 & 406.9 & 271 & 119 & 163 & 15.2 & 10.1 \\
\hline \multicolumn{8}{|c|}{ Warren [6], 3P (A) and $4 \mathrm{P}(\mathrm{B})$} \\
\hline $1 A$ & 3100 & 289.8 & 200 & 133.4 & 100 & 7.8 & 52.8 \\
\hline $1 B$ & 5500 & 289.8 & 200 & 133.4 & 100 & 7.8 & 5.8 \\
\hline $2 A$ & 3800 & 309.3 & 225 & 133.4 & 75 & 7.8 & 5.8 \\
\hline $2 B$ & 5600 & 309.3 & 225 & 133.4 & 75 & 7.8 & 5.8 \\
\hline $3 A$ & 3800 & 435.0 & 300 & 101.6 & 150 & 6.8 & 5.8 \\
\hline $3 B$ & 4200 & 435.0 & 300 & 101.6 & 150 & 6.8 & 5.8 \\
\hline $4 A$ & 8200 & 463.2 & 325 & 101.6 & 75 & 6.8 & 5.8 \\
\hline $4 B$ & 7400 & 463.2 & 325 & 101.6 & 75 & 6.8 & 5.8 \\
\hline
\end{tabular}

\subsection{Characteristics of the finite element model}

The 4-node shell element SHELL181 was used to simulate the beam's plates (web, flanges, and web stiffeners) and additional unidimensional elements (BEAM188 and LINK180) were used to prevent distortion at the supports. The steel was modeled adopting either a bilinear or a multilinear stress-strain curve, following, for each experiment simulated, the material specifications adopted by the authors responsible for the experiment. The analyses were solved using an arc-length technique (which allows the study of the post-failure behavior) combined with geometric and physical nonlinearities and large displacement 
theory. The point loads were spread along the width of the top flange and applied as a set of nodal forces. As a result of mesh refinement studies the size of the elements in each model was taken to be the smallest value given by the five following expressions: $s_{w} / 4, b_{f} / 4, d_{t} / 3, s_{d} / 8$, and $L / 100$ (in which $d_{t}$ is the height of the web above the center of the opening, and $s_{0}$ is the width of the opening).

\subsection{Calibration of the numerical model}

The experimental and numerical results extracted from the literature (compiled in Table 1 and Table 2) were compared with the numerical results obtained in the proposed numerical model. This comparison was made using three criteria to evaluate the quality of the simulation: the maximum strength of the beam, the shape of the load-displacement curve, and the failure mode (visually identified based on the deformed shape). No residual stresses were applied in this study, even though Sonck [2] and Vieira [8] did consider them in their numerical analyses. The geometric imperfections included in the proposed numerical model matched the ones adopted by the authors of each reference model in their own numerical analyses, both in shape and in magnitude. The only exceptions to this approach were the two models by Nseir et al. [4], in which a sophisticated method was developed to measure the initial geometry of the beam and transfer it to the numerical model. Since this could not be replicated, both models (HEA_340 and IPE_330) were given an initial imperfection with the shape of global bending about the weak-axis and magnitude of $L / 1000$.

The load ( $\mathrm{kN}$, vertical axis) versus displacement at mid span ( $\mathrm{mm}$, horizontal axis) curves for some of the reference models are presented in Fig. 5. The thick continuous lines represent the results obtained in the proposed numerical model, the dashed lines represent the experimental results, and the thin continuous lines represent the numerical results obtained by the authors who carried out the experimental analyses.
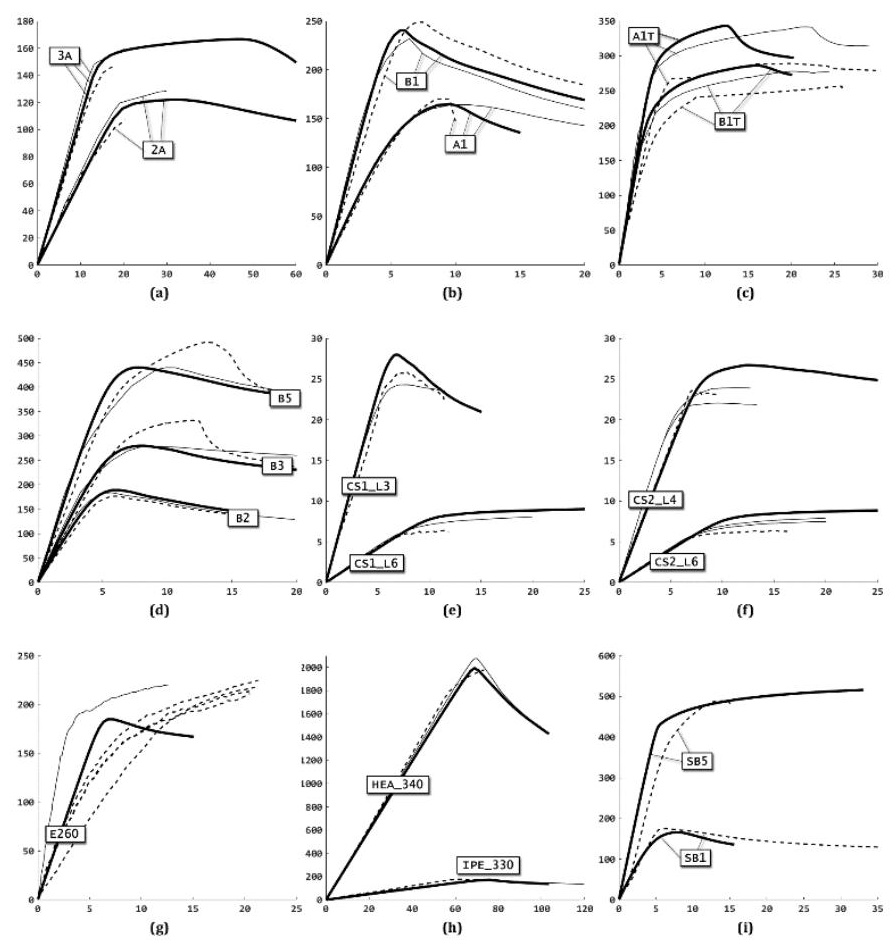

Fig. 5 Validation of the numerical model: load-displacement curves for selected beams

In general the numerical and experimental curves display good agreement. There are, however, some important differences. Model CS1_L6 (Fig. 5-e), for instance, deviated from the experimental results and predicted higher failure loads. The same tendency was observed by Sonck [2], and indeed the numerical curves (continuous lines) display matching behavior. Sonck [2] attempted to explain this discrepancy by citing difficulties with the loading equipment, which caused premature interruption of the experiment.

For model $A I T$ (Fig. 5-c) the numerical curves match each other but diverge from the experimental results in a significant manner. Tsavdaridis \& D'Mello [9] considered this result a satisfactory calibration of the numerical model. Model $B 3$ (Fig. 5-d), presented by [8], displays a similar phenomenon, with the numerical results agreeing with each other but diverging from the experimental result. Vieira [8] also considered this result satisfactory. All the numerical results corresponding to the reference models by [1] displayed very poor agreement, even though only model E260 (Fig. 5-g) is presented. Even the author's own numerical analysis failed to match the experimental results. This indicates possible problems with the experimental design or with its reporting.

The remaining models presented very good agreement between numerical and experimental results, leading to the conclusion that the proposed numerical model simulates well the behavior of castellated and cellular beams. Some of the differences between numerical and experimental results observed in this calibration might be due to inadequate assumptions made in the construction of the numerical model. One of these assumptions, namely the shape of the initial geometric imperfection, is investigated further in this paper.

\section{Study of the different shapes of geometric imperfection}

The influence of the four types of geometric imperfection presented previously (Fig. 3 and Fig. 2-b) on the behavior of castellated and cellular beams was analyzed. The numerical results obtained will always depend upon the magnitude of the geometric imperfection introduced, which, in turn, displays great variability in real world beams $[8,4]$. In an attempt to isolate the effects of the shape of the imperfection rather than that of its magnitude on the numerical model all the simulations were performed using fixed expressions for the determination of the size of the imperfection introduced. These expressions are listed in Table 3 and were chosen based on generally accepted guidelines, such as those provided by Eurocode 3 [20].

Table 3

Shapes and magnitudes of the geometric imperfections studied with the numerical model

\begin{tabular}{ccc}
\hline Shape & Code & Magnitude \\
\hline Weak-axis bending & FW & L/1000 \\
Web bending & WB & $d_{g} / 200$ \\
Buckling mode (WBS) & BM & $d_{g} / 200$ \\
Web-post misalignment & PM & Smallest between $d_{g} / 200$ and $t_{w} / 200$ \\
\hline
\end{tabular}

Due to the adoption of the fixed parametric expressions the magnitudes of the geometric imperfections no longer display any correspondence with the experimental values, used during validation. The imperfection magnitudes that were altered the most were those of the two models by Tsavdaridis \& D'Mello [9] (A1T and B1T), since the authors adopted a magnitude of $0.038 \mathrm{~mm}$ while the parametric expressions in Table 3 result in a magnitude of $2.19 \mathrm{~mm}$, a value over 50 times greater.

\subsection{Weak-axis bending $(F W)$}

The weak-axis bending, when applied to the most slender and unbraced beams, resulted in the occurrence of LTB. However, when applied to shortspanned, laterally braced beams, this same imperfection shape did not significantly affect the stability of the web-post. Fig. 6 displays loaddisplacement curves of three models simulated in ANSYS either with weak-axis bending as geometric imperfection or with no geometric imperfections.

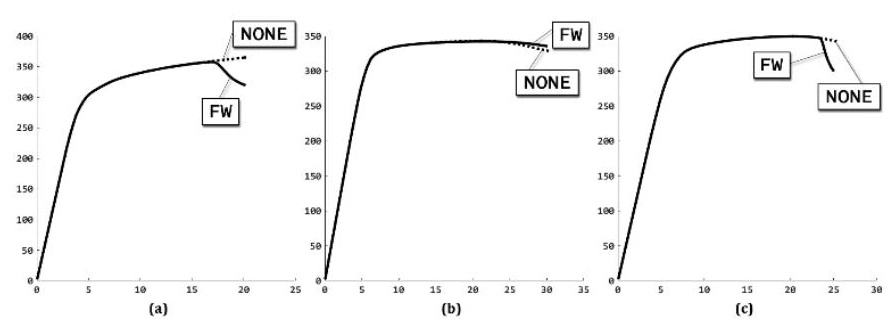

Fig. 6 A1T (a), B3 (b), and SB3 (c): global and no imperfections yielding similar results

The similarity between the pairs of curves in Fig. 6 reveals the small influence the weak-axis bending has over the behavior of short-spanned and laterally braced beams. Only after the structure has reached a high degree of plastic yielding do the two curves diverge, a behavior that has been observed in most of the beams simulated.

In some cases, however, the small influence of the global imperfection was enough to generate web-post instability. Fig. 7 displays the results of model B2, in which the occurrence of instability can be observed at the end of the linear segment of the load-displacement curve. The deformed shapes (taken at the poin of maximum load for each curve) reveal the occurrence of WBS in the model with the imperfection and the occurrence of plastic yielding (Vierendee 
mechanism and longitudinal shearing of the web-post) in the model without imperfections.

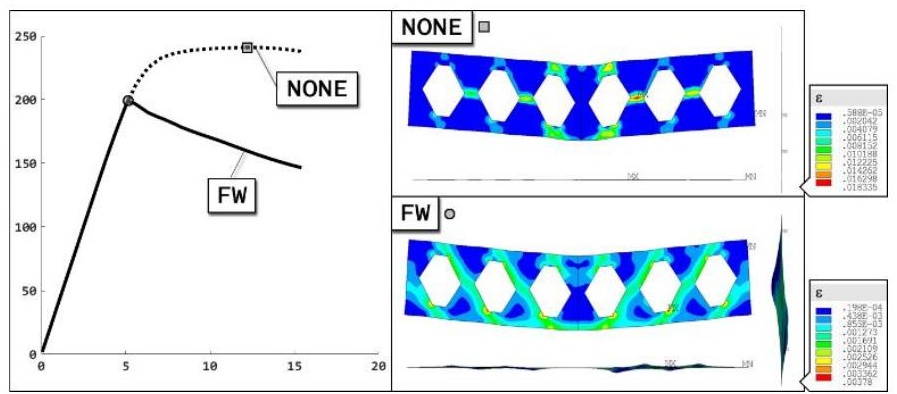

Fig. 7 Model B2: global imperfection (FW) impacting web-post stability

\subsection{Web bending $(W B)$}

The bending of the web is present in plain-webbed I-sections, but amplified by the fabrication process of cellular and castellated beams. The presence of this imperfection generates eccentricities in the centroid and shear center of the crosssection with respect to the direction of the vertical load, which leads the applied shear forces to generate torsion in the beam. In a second-order analysis of slender beams this small torsional rotation can develop into lateral-torsional buckling. For short-spanned beams the curved shape of the web-post can lead to instability, either caused by axial compression of the web-post or by diagonal compression due to shear forces.

The results obtained when web bending was introduced as geometric imperfection can be summarized as follows:

- Slender, laterally unbraced beams: collapse by lateral-torsional buckling (Fig. 8);

- $\quad$ Short-spanned beams without web stiffeners: the web-post directly under the load introduction point buckled by compression (Fig. 9);

- $\quad$ Short-spanned beams with web stiffeners: collapse happens either by WBS (Fig. 10), by longitudinal shear of the web-post (Fig. 11), or by Vierendeel mechanism (Fig. 12).

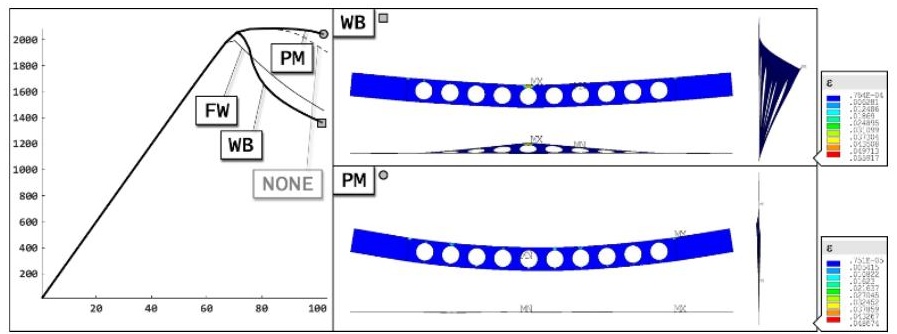

Fig. 8 Model HEA_340: collapse by either LTB or plastic yielding

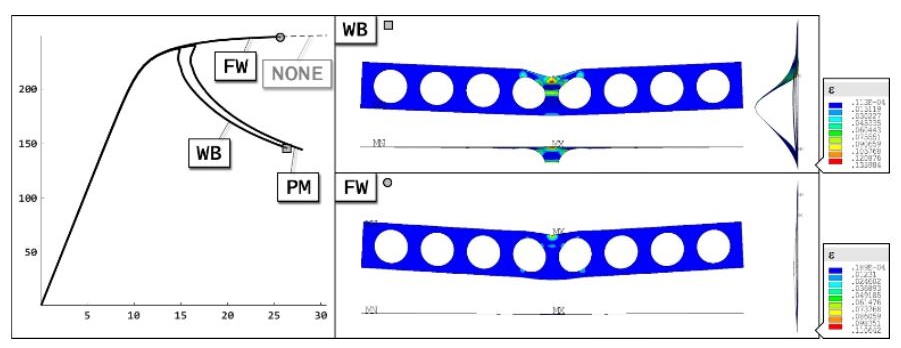

Fig. 9 Model E24OC: collapse by either WBC or plastic yielding

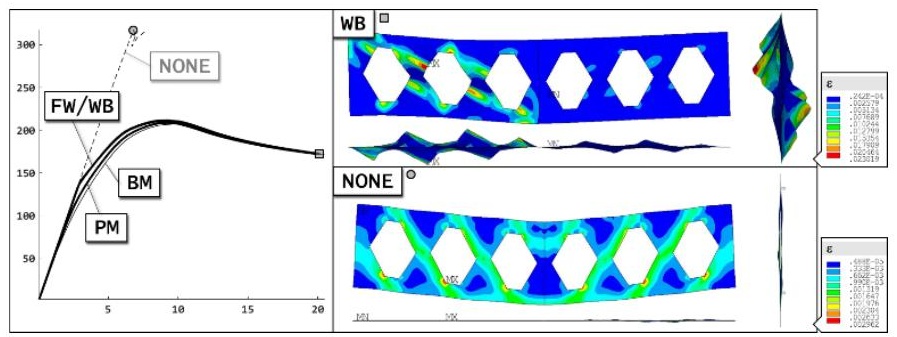

Fig. 10 Model SE1: collapse by WBS when any geometric imperfection is introduced

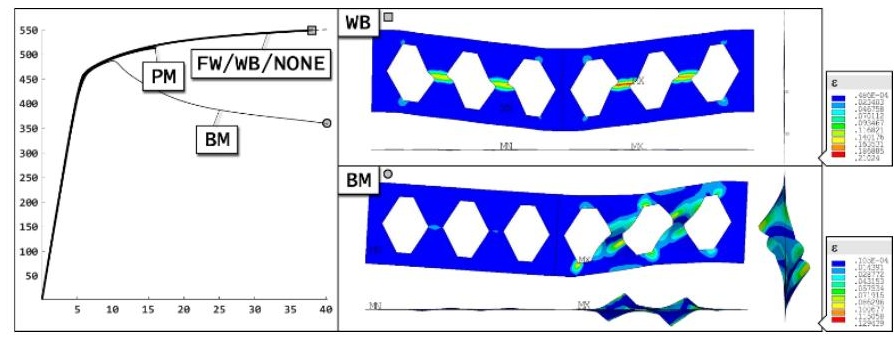

Fig. 11 Model B5: collapse by either WBS or LS

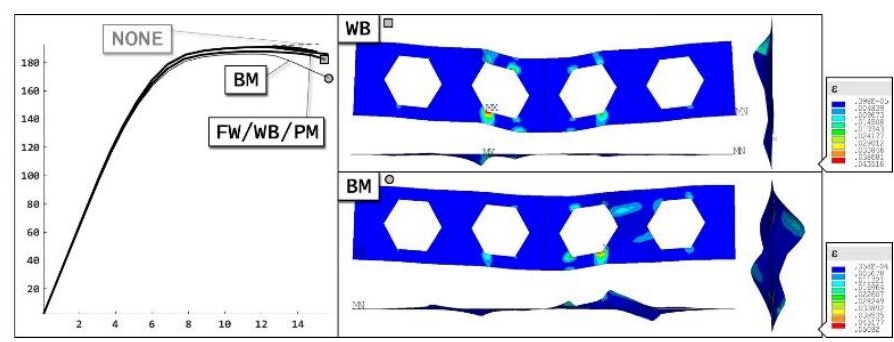

Fig. 12 Model A2: collapse by either VM or WBS

\subsection{Buckling mode shape (BM)}

The shape of the web-post buckled due to shear force was obtained in an eigenvalue analysis to then be used as geometric imperfection in the nonlinear analysis for the study of post failure behavior. This buckling mode, however, tends to occur only in short-spanned, laterally braced beams. Longer beams will display global instability, and attempts to artificially constrain the slender beam in order to facilitate the occurrence of the WBS buckling mode were unsuccessful. Therefore, the buckling mode shape was applied only to the short-spanned beams, for which the eigenvalue analysis was able to generate the desired geometric imperfection shape.

Introducing the deformed shape obtained in the eigenvalue analysis as geometric imperfection is the conventional approach, often adopted by researchers studying the problem of collapse by WBS [8.9]. In all the simulations performed the introduction of the buckling mode shape eventually led to collapse by web-post buckling due to shear (WBS), as can be seen in Fig. 11, Fig. 12, Fig 13, and Fig. 14.

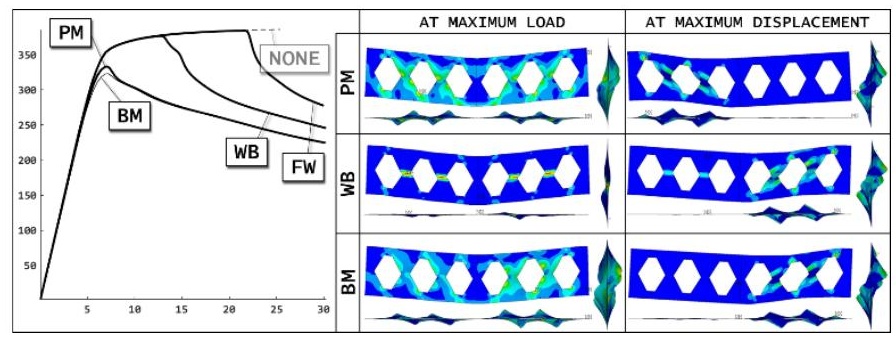

Fig. 13 Model B4: collapse by either WBS or WBS and LS

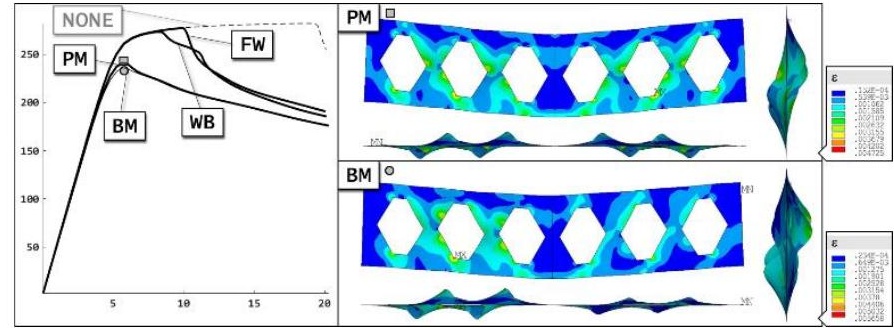

Fig. 14 Model B1: collapse by WBS

4.4. Web-post misalignment (PM)

In the standard fabrication process for castellated and cellular beams it is impossible to assure perfect alignment between the upper and lower halves of the beam during welding. To simulate this misalignment of the web in the numerical 
model the cross-sections of the shell elements in the top and bottom halves of the web were offset from the midplane in opposite directions. This approach introduces small eccentricities, but no mesh discontinuities. Due to its ease of application as a geometric imperfection the web-post misalignment was tested in all the beams studied.

The introduction of the web-post misalignment led the slender beams to collapse by global instability (LTB) in all cases except one. Model HEA_340 was this single exception, having collapsed due to the formation of a plastic hinge caused by bending moment (Fig. 8).

For short-spanned, laterally braced beams with web stiffeners the introduction of web-post misalignment led to collapse by WBS (Fig. 13, Fig. 14, and Fig. 15).

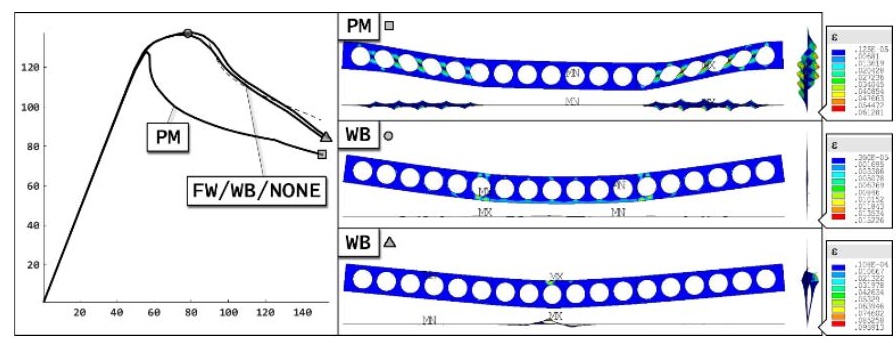

Fig. 15 Model 4B: collapse by either WBS or PH

The long-spanned but laterally braced beams (most of the beams studied by Warren [6]) collapsed due to the formation of plastic hinges caused by bending moment and shear force (Fig. 16). Short-spanned beams without web stiffeners collapsed by web-post buckling due to compression (WBC, Fig. 9).

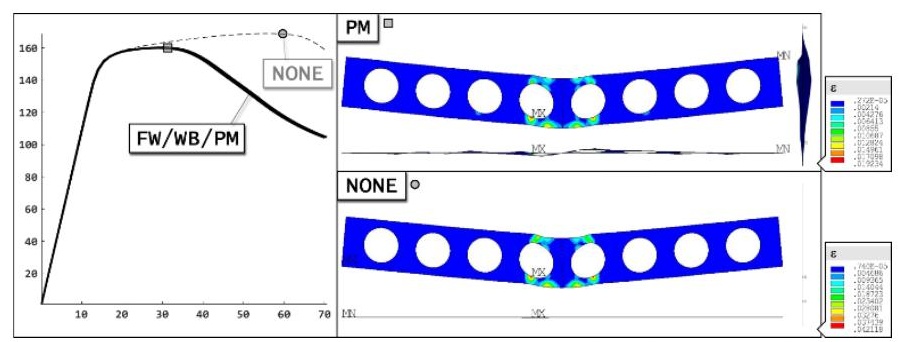

Fig. 16 Model 3A: collapse by formation of plastic hinges (PH)

\section{Discussion of the results}

The simulations performed can be roughly divided into two groups: beams that fail by global instability and beams that fail by other mechanisms. The longspanned unbraced beams $\left(C S 1 \_L 3, C S 1 \_L 4, C S 1 \_L 6, C S 2 \_L 3, C S 2 \_L 4, C S 2 \_L 6\right.$, $I P E \_330$, and $E 240 A$ ) fail by lateral-torsional buckling as long as any of the studied geometric imperfections is applied. Despite that, a comparison of the load-displacement curves indicates that different imperfection shapes lead to different ultimate resistances (Fig. 8, Fig. 17, and Fig. 18). The geometric imperfection that most reliably produces the lowest resistance against LTB is the global flexure about the weak axis of inertia. This result was expected and is in accordance with conventional approaches, that recommend the use of the first buckling mode as imperfection shape. The flexure about the weak axis does not match the LTB shape exactly for not including the torsional deformation, but it is a very close analogue.

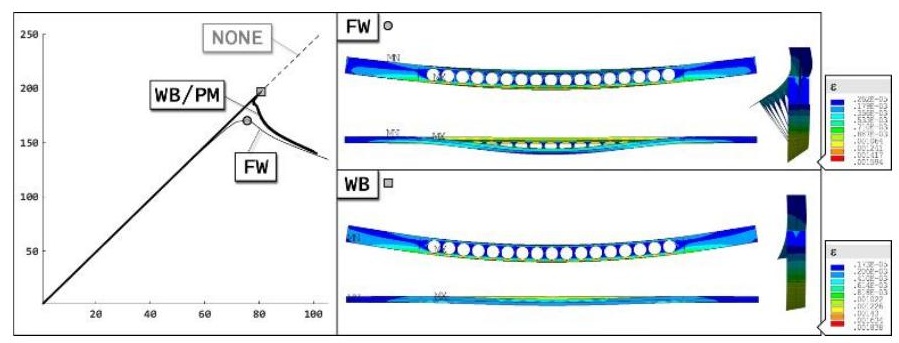

Fig. 17 Model IPE_330: collapse by LTB

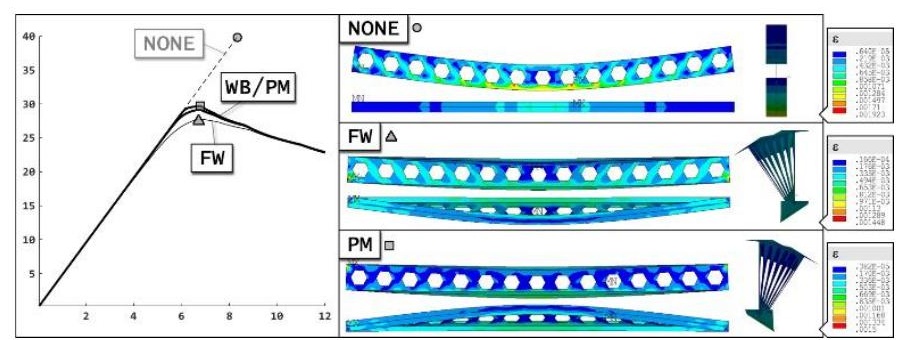

Fig. 18 Model CS1_L3: collapse by LTB when imperfections are introduced

The geometric imperfections that affect primarily the web (web-post bending and web-post misalignment) have also triggered LTB, but usually allowed the simulations to reach higher loads and led to more abrupt transitions between the linear elastic and the post-failure regimes. This abrupt transition can be observed in the load-displacement curves at the point of maximum load, especially when contrasting models with web imperfections against models with the global imperfection (Fig. 17).

In one case the introduction of the web-post misalignment was insufficient to provoke LTB and beam HEA_340 failed by plastic yielding (Fig. 8). This indicates that, at least for the magnitudes studied, the introduction of web imperfections may not be sufficient to trigger global instability in long-spanned, laterally unbraced beams. The introduction of global imperfections (first buckling mode or flexure about the weak axis of inertia) is therefore recommended.

The remaining beams simulated were laterally braced to prevent global instability. These beams displayed a great variety of failure modes, that were directly affected by the imperfections introduced. The introduction of the global imperfection (flexure about the weak-axis) caused very little effect, and many of the beams still behaved as if no geometric imperfection had been introduced. Due do its lack of impact in the models this imperfection will be excluded from further analysis.

The three remaining geometric imperfection shapes (web bending, WBS buckling mode, and web-post misalignment) produced clear and significant impacts in the structural behavior of the beams. The dominant modes of failure observed in the numerical model were web-post buckling due to shear (WBS), web-post buckling due to compression (WBC), Vierendeel mechanism (VM), longitudinal shear at the web-post (LS), and plastic hinges due to bending ( $\mathrm{PH})$. Each of these modes has clear definitions in the literature [5,6,10], but they do not always occur in isolation. These failure modes can be grouped into two categories: collapse by instability (WBS, WBC) and collapse by plastic yielding (VM, LS, PH).

To better comprehend the types of collapse that occurred in the numerical model both the deformed shape and the first principal strain pattern were carefully inspected. This was necessary because beams that displayed very similar deformed shapes, often those characteristic of collapse by instability, could also have strain patterns that pointed to different modes of collapse by plastic yielding. The first principal strain was found to be the quantity best suited to tease out plastic yielding in beams dominated by web-post instability. As a result of this approach the beams that displayed web-post buckling due to shear were further divided into three groups:

- $\quad$ pure WBS: no signs of any mode of failure except web-post buckling due to shear (Fig. 10, Fig. 13, Fig. 14, and Fig. 15);

- WBS+LS: simultaneous presence of WBS and longitudinal shear at the web post (Fig. 13);

- WBS+VM: simultaneous presence of WBS and Vierendeel mechanism (Fig. 12).

The different types of collapse (instability and plastic yielding) are also reflected in the shape of the load-displacement curve. Fig. 19 presents three loaddisplacement curves, each characteristic of one type of collapse. Type A curve is associated with collapse by instability. At the end of the linear-elastic segment the model experiences a short curved segment, at the end of which the maximum load is reached. After this point the displacements grow quickly and the load decreases. Type $B$ curve is associated with collapse by plastic yielding. The linear-elastic segment is followed by a short curved segment that leads into a new linear segment, less steep than the first one and sometimes approaching a plateau. Development of plastic yielding occurs in the second linear segment. The displacements grow quickly while the load grows at a much slower rate. The curve ends at the point of maximum load, when the finite element model can no longer find stable configurations for the beam. Type $C$ curve combines the two phenomena, with instability following plastic yielding. At first the beam suffers some sort of plastic yielding as indicated by the second ascending linear segment, but this regime is ended by the occurrence of instability, characterized by the sudden decrease in applied load. 


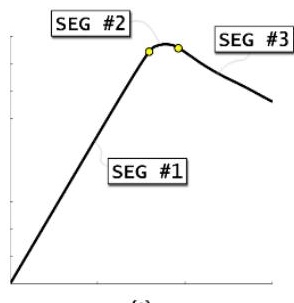

(a)

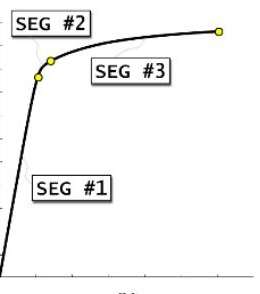

(b)

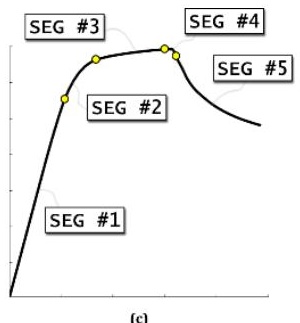

(c)
Fig. 19 Shapes of load-displacement curves associated with different types of collapse

Given the types of collapse described above the effect of each geometric imperfection can be analyzed. Table 4 summarizes the results of the numerical analyses performed. For beams that fail by web-post buckling due to shear (WBS) the results indicate that the collapse is more likely to be accompanied by some sort of plastic yielding when web bending is introduced as geometric imperfection. When either web-post misalignment or the first buckling mode are introduced the beams are pushed towards experiencing pure WBS.

Table 4

Types of collapse depending upon the shape of gometric imperfection introduced

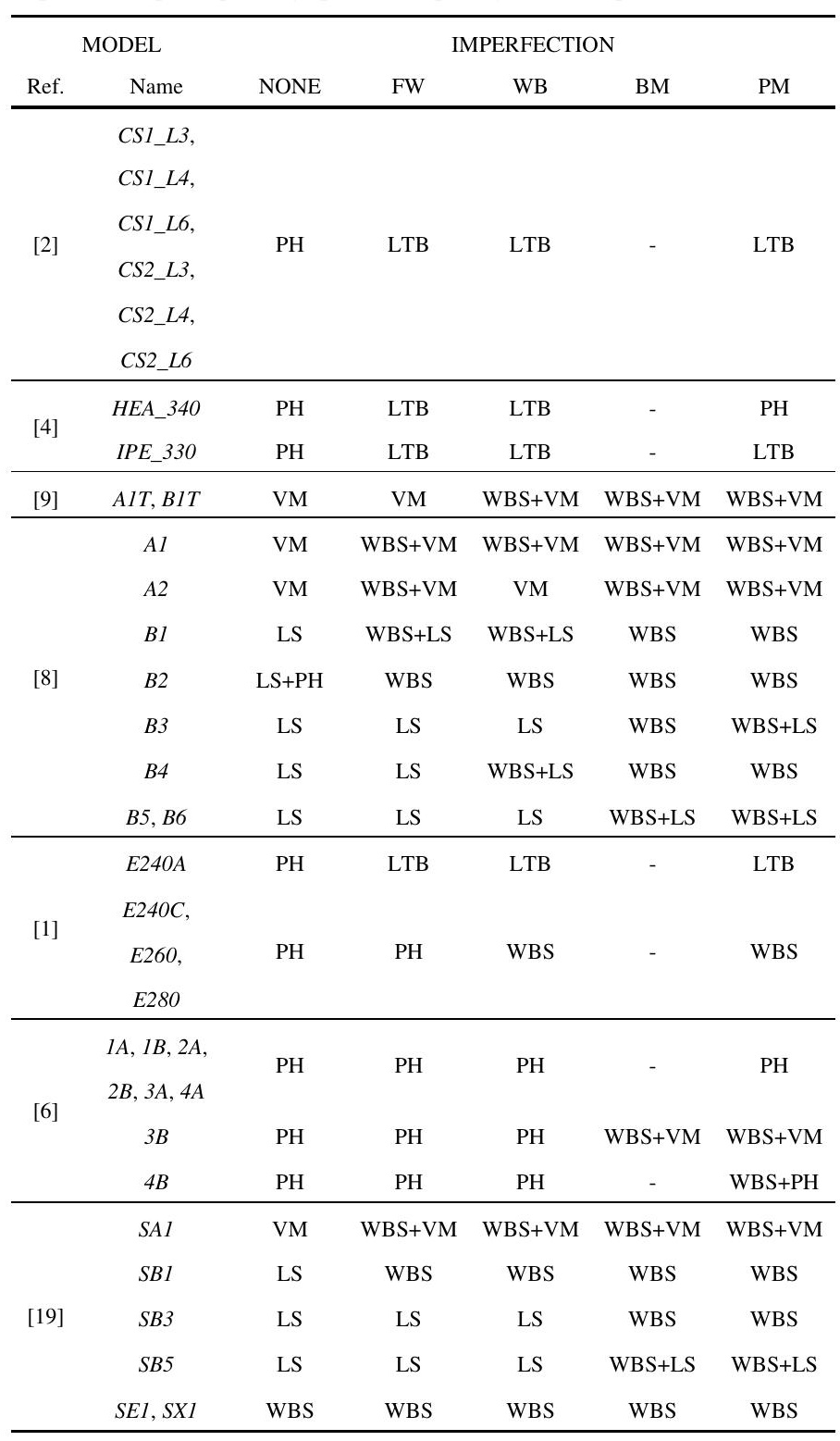

Models $B 4$ (Fig. 13) and SB3 (Fig. 20) provide the clearest examples of the effect of different shapes of geometric imperfection. The shape of the loaddisplacement curves indicates that beams with either web-post misalignment or first buckling mode as geometric imperfection failed by web-post instability. Inspection of the first principal strain patterns (highest values form narrow diagonal bands) confirms pure WBS. The beams with web bending, on the other hand, display load-displacement curves that indicate the occurrence of plastic yielding followed then by web-post instability. Inspection of the first principal strain patterns corroborates this conclusion and also reveals that both beams display a pattern characteristic of longitudinal shear at the web-post (maximum strain values form horizontal bands at the center of the web-post).

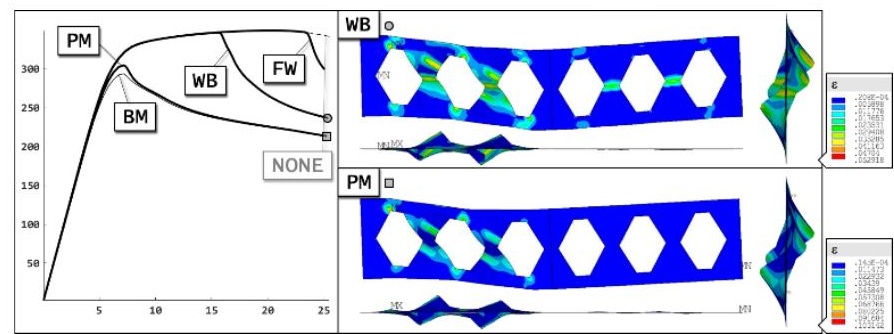

Fig. 20 Model SB3: collapse by WBS, sometimes accompanied by LS

As the study of models $B 4$ and SB3 shows, the choice of geometric imperfection shape significantly affects the results of the simulation, both qualitatively (changing the failure modes) and quantitatively (web bending led to higher overall resistance). While the adoption of the first buckling mode as a geometric imperfection might be justified on grounds of erring on the side of caution, a comparison with experimental results indicates that this approach might lead to overly conservative resistance values or even to an incorrect prediction of the failure mode for castellated and cellular beams. Fig. 21 presents a case in which the use of WBS buckling mode as geometric imperfection (BM) leads to a disparity of this nature between numerical simulation and experimental results, with the numerical simulation predicting collapse by WBS while the experimental curve indicates development of plastic yielding before instability occurs.

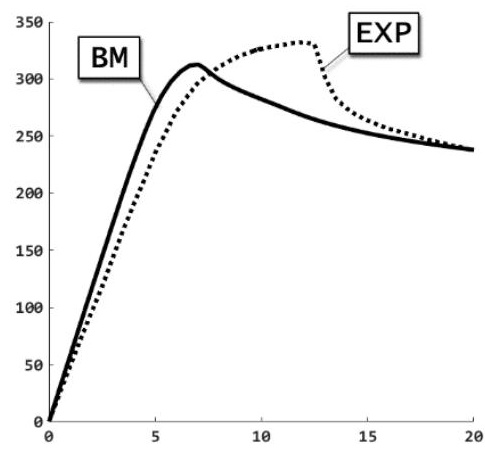

Fig. 21 Model B3: mismatch between numerical simulation and experimental result

\section{Conclusions}

Simulating the structural behavior of castellated and cellular beams using finite element models requires an accurate understanding of the influence of real world deviations in geometry. This paper studied, using a properly calibrated numerical model, the effects of four different types of geometric imperfection: weak-axis bending (FW), web bending (WB), WBS buckling mode (BM), and web-post misalignment (PM). The magnitudes of the imperfections were fixed at $L / 1000$ for the global imperfection (FW) and at $d_{g} / 200$ for the local imperfections. The conventional approach (introducing the shape obtained in an eigenvalue buckling analysis as geometric imperfection) was contrasted with the novel approach of introducing imperfection shapes that more closely resemble experimental measurements.

The global imperfection was found to have an effect only in long-spanned, laterally unbraced beams, in which it triggered collapse by lateral-torsional buckling. Short-spanned, laterally braced beams were mostly unaffected by weak-axis bending. The local imperfections (WB, BM, and PM) also led to LTB when applied to slender beams. In general, however, the introduction of the global imperfection more reliably led to LTB and to lower overall resistances, and therefore the conventional approach is recommended for slender beams.

It is important to note that the WBS buckling mode (BM) could not be applied to most slender beams. This was due to the fact that its introduction requires that it first be obtained in an eigenvalue analysis, which could not be done in most cases. This limitation, characteristic of the conventional approach, means the engineer has little control over the shape of the geometric imperfection 
introduced.

For laterally braced beams the introduction of web-based imperfections (WB $\mathrm{BM}$, and PM) led to a great variety of modes of failure, with results heavily dependent upon the geometry of the beams. Short-spanned, laterally braced beams manifested most clearly the influence of the shape of geometric imperfection. In these beams the lack of imperfection led to collapse by plastic yielding (longitudinal shear at the web-post or Vierendeel mechanism), but the introduction of web-based imperfections affected the stability of the web-post.

The web bending (WB) had the weakest effect on web-post stability. Its introduction usually led to collapse that was either entirely plastic (LS or VM) or a form of WBS heavily tainted with plastic yielding (WBS+LS or WBS+VM). The development of plastic yielding followed by web-post buckling due to shear (WBS) can be detected in the shape of the load-displacement curve (Fig. 19) and in the patterns formed by the first principal strain (Fig. 20).

The web-post misalignment (PM) and the WBS buckling mode (BM) produced very similar effects on short-spanned, laterally braced beams. Both imperfections strongly affected the web-post stability, with the buckling mode compromising it the most. In most cases the difference between PM and BM was hard to detect, but in a few beams (Fig. 11 and Fig. 12) the introduction of the web-post misalignment allowed greater development of plastic yielding before triggering collapse by WBS.

The web-post misalignment and the buckling mode (WBS-shape) can, the results suggest, be adopted interchangeably. This means that the structural behavior changes triggered by the WBS-shape, that were once restricted to geometries for which this imperfection could be obtained in an eigenvalue analysis, can now be included in any castellated or cellular beam through the introduction of the web-post misalignment, an imperfection that can be easily and universally applied.

\section{Acknowledgments}

The authors wish to thank CPNq, CAPES, and FAPEMIG for the financial support provided for the development of this research study.

\section{References}

[1] F. Erdal, Ultimate load capacity of optimally designed cellular beams, Ph.D. thesis, Middle East Technical University (2011)

[2] D. Sonck, Global buckling of castellated and cellular steel beams and columns, Ph.D. thesis Ghent University (2014)

[3] N. Boissonnade, J. Nseir, M. Lo, H. Somja, Design of cellular beams against lateral torsional buckling, Proceedings of the Institution of Civil Engineers - Structures and Buildings 167 (2014) 436444. doi:10.1680/stbu.12.00049.

[4] J. Nseir, M. Lo, D. Sonck, H. Somja, O. Vassart, N. Boissonnade, Lateral torsional buckling of cellular steel beams, Proceedings of the Annual Stability Conference - Structural Stability Research Council (2012)

[5] R. M. Lawson, S. J. Hicks, Design of Composite Beams with Large Web Openings, SCI, 2011

[6] J. Warren, Ultimate load and deection behaviour of cellular beams, Master's thesis, School of Civil Engineering, Surveying and Construction - University of Natal (2001).

[7] K. Chung, T. Liu, A. Ko, Investigation on vierendeel mechanism in steel beams with circular web openings, Journal of Constructional Steel Research 57 (5) (2001) 467490 doi:https://doi.org/10.1016/S0143-974X(00) 00035-3.

[8] W. B. Vieira, Estudo numérico-experimental da ambagem do montante de alma em vigas casteladas de aço, Ph.D. thesis, Universidade Federal de Viçosa (2015)

[9] K. D. Tsavdaridis, C. D'Mello, Web buckling study of the behaviour and strength of perforated steel beams with dierent novel web opening shapes, Journal of Constructional Steel Research 67 (10) (2011) 1605 1620. doi: https://doi.org/10.1016/j.jcsr.2011.04.004.

[10] D. Kerdal, D. Nethercot, Failure modes for castellated beams, Journal of Constructional Steel Research 4 (4) (1984) 295 - 315. doi:https://doi.org/10.1016/0143-974X(84)90004-X.

[11] Guo J., Research on distribution and magnitude of initial geometrical imperfection affecting stability of suspen-dome, Advanced Steel Construction Vol. 7, No. 4, pp. 344-358 (2011).

[12] Zhao Z. W., Liu H. Q., Liang B., Yan R. Z., Influence of random geometrical imperfection on the stability of single-layer reticulated domes with semi-rigid connection, Advanced Steel Construction Vol. 15, No. 1 (2019) 93-99. DOI: 10.18057/IJASC.2019.15.1.12.

[13] Masri O. Y. E., Lui E. M., Influence of imperfections on the flexural resistance of steel delta girders, Advanced Steel Construction Vol. 15, No. 2 (2019) 157-164. DOI: 10.18057/IJASC.2019.15.2.5.

[14] E. Ellobody, Nonlinear analysis of cellular steel beams under combined buckling modes, ThinWalled Structures 52 (2012) 66 - 79. doi: https://doi.org/10.1016/j.tws.2011.12.009.

[15] E. Ellobody, Interaction of buckling modes in castellated steel beams, Journal of Constructiona Steel Research 67 (2011) 814 - 825. doi: https://doi.org/10.1016/j.jcsr.2010.12.012.

[16] Gehring A., Saal H., Numerical analyses of cold-formed thin-walled sections with consideration of imperfections due to the production process, Advanced Steel Construction Vol. 5, No. 2, pp. 151-163 (2009).

[17] ArcelorMittal, Angelina beams - A new generation of castellated beams (2016).

[18] I. ANSYS, ANSYS Mechanical APDL, Release 14.0 - Help System (2011).

[19] G. S. Veríssimo, E. K. H. Sakiyama, J. L. R. Paes, J. C. L. Ribeiro, R. H. Fakury, R. B. Caldas, W. B. Vieira, Estudo numérico-experimental do desempenho estrutural de vigas alveolares soldadas, Tech. rep., Universidade Federal de Viçosa (2017).

[20] EN, EN 1993-1-5 Eurocode 3: Design of steel structures - Part 1-5: General rules - Plated structural elements, CEN, Brussels, 2006. 


\title{
ANALYSIS OF BEAM-TO-UPRIGHT END CONNECTIONS STEEL STORAGE SYSTEMS
}

\author{
Lucas Alves Escanio, Guilherme Cássio Elias, Luiz Henrique de Almeida Neiva, \\ Vinícius Nicchio Alves and Arlene Maria Cunha Sarmanho*
}

\section{A B S T RA C T}

This study presents an experimental and numerical research into the behavior of beam-to-upright end connections, which are part of steel storage systems. A total of 21 cantilever tests comprising three upright and four beam cross sections, were conducted, and a finite element numerical model was developed. The model was developed to compare its results with experimental results and to extrapolate these results in a parametric analysis. The aim of the study is to evaluate the behavior, semi-rigidity, and influence of the geometric parameters in the stiffness of beam-upright lipped connections of pallet-type industrial storage systems. The results of the numerical model showed good correlation with the experimental results, in terms of moment-rotation behavior and the initial stiffness values, with a maximum difference of $10 \%$. In the parametric analysis, a pattern was observed in the variation of stiffness when the dimensions - mainly the height of beams-of the prototypes were increased.

\section{A R T I C LE H I S T O RY}

$\begin{array}{ll}\text { Received: } & 3 \text { October } 2019 \\ \text { Revised: } & \text { 22 June } 2020 \\ \text { Accepted: } & \text { 22 June } 2020\end{array}$

\section{K E Y W O R D S}

Cold-formed steel

Storage rack uprights;

Cantilever test;

Semi-rigid connection

Beam-to-upright lipped connection

\section{Introduction}

Industrial storage systems are traditionally used in various industries to store different types of manufactured products. These storage systems are produced in cold formed profiles. The uprights have sections specially developed to facilitate their assembly, containing perforations along their length to facilitate the connection with the beams. These systems provide great practicality, offering several configurations of storage arrangements considering the characteristics of each product. A rectangular hollow section is commonly used for the beams, and they are positioned in the structure perpendicular to the plane of the uprights with the purpose of receiving the load from the pallets and transmitting it to the uprights and bases by means of the connections

The connections between beams and uprights are semi-rigid. In the case studied in this work, the end connectors are formed by a plate containing lips, and they are placed at the beam's ends. The lips of the plate fit into the holes of the upright, which are evenly distributed along its length, to create unity between the upright and the beam [1]. The details of the beam and the lipped connector in this study are presented in Fig. 1 and Fig. 2.

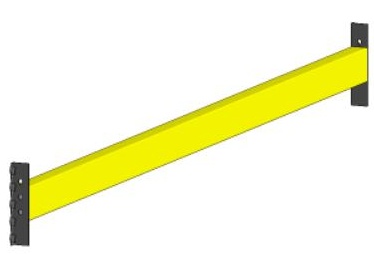

(a)

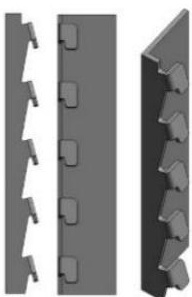

(b)
Fig. 1 (a) Beam with connectors; (b) The end connector studied

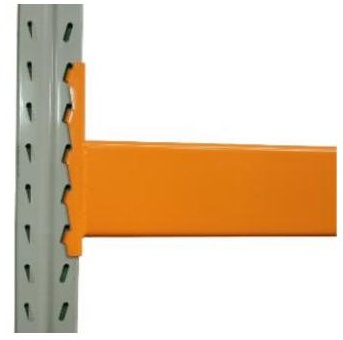

Fig. 2 Perforations and upright-beam connection

Shah et al. [2] performed an extensive study on state-of-the-art pallet rack connections of steel. The authors concluded that parameters such the height of the pallet beam, the column's thickness and the connector's geometry affects the strength and stiffness of the connector. Several numerical and experimental studies [3-5] have been carried out to investigate the influence of perforations on the upright's stability and load capacity.

Bajoria and Talikoti [6] determined the flexibility of beam-to-column connectors used in thin-walled cold-formed steel pallet racking systems by two different ways. The standard cantilever method and the proposed double cantilever method were used, and it was verified that to determine the flexibility of the connector, the double cantilever procedure can be used instead of the conventional cantilever test.

Based on experimental and numerical studies on the flexibility of the connection in cold formed steel racks, Prabha et al. [1] observed that the moment rotation characteristic of a connection is determined by the efficiency of the connection members (specially the upright) and by the design of the beam end connector. Furthermore, the increased height of the beam and thickness of the upright also increased the strength and stiffness of the joint.

Cardoso and Rasmussen [7] developed a finite element model to study storage rack frames, which used a bi or multi-linear moment rotation curve for the upright-pallet beam joints. They observed that the present component tests for rack frames connections overestimated the joint stiffness and were unable to emulate the behavior of the connection at the system level. The stiffness and semi-rigidity of the connector were also tested by Bajoria et al. [8], using the standard cantilever method and a double cantilever method. In addition, a finite element modal analysis of both tests was carried out. The proposed mathematical model was verified for single-, two-, and six-bay frames, with and without mass. All the frame results were very close to the free vibration modal analysis result. That means that this model could be used for any number of bays.

Several studies in the literature aim to study the cold-formed steel uprights and the connection between the upright and beams using experimental knowledge and numerical modeling [3,5,9-15].

In this study, the developed numerical model was calibrated with the experimental results to evaluate the behavior, semi-rigidity, and influence of the geometric parameters on the stiffness of beam-upright lipped connections of pallet-type industrial storage systems. Numerical and experimental studies were performed to determine the moment-rotation curve and connection stiffness. A total of 7 combinations of connection between beam and uprights with different cross-sections were tested in the experimental study. For each combination, 3 experimental tests were performed, resulting in 21 experimental tests.

A numerical finite element analysis of the 7 combinations was performed using the ANSYS software [16]. The numerical models were validated by comparing their behavior and stiffness with the experimental results, and the influence of each lip on the behavior of the connection was studied. A parametric study was carried out to evaluate the effects of the variation of the 
geometric parameters of the elements of the structural assembly on the connection's stiffness.

\section{Experimental program}

\subsection{Cross-section geometry}

A total of 21 cantilever tests [17] were conducted in this study to determine the stiffness and the bending strength of the lipped connection. Three upright and four beam cross sections were considered, totalizing seven combinations. The three different uprights considered for the cross section of the rack-type uprights had different web widths, $b_{w}=80,90$, and $100 \mathrm{~mm}$. The considered upright cross-sectional thickness was $t=2.00 \mathrm{~mm}$, and the cross-sections are shown in Fig. 3 with the dimensions in Table 1.

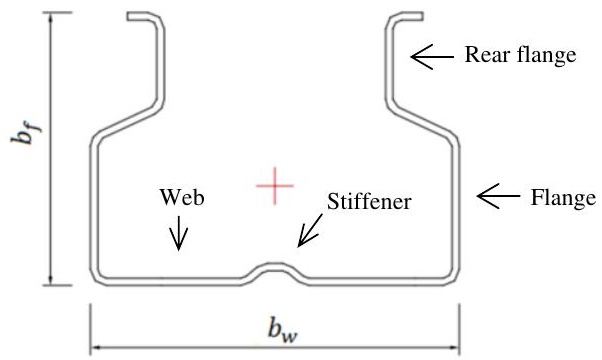

Fig. 3 Cross section considered for uprights with the elements identified

Table 1

Dimensions of upright cross section

\begin{tabular}{ccccc}
\hline Upright & Length $(\mathrm{mm})$ & $t(\mathrm{~mm})$ & $b_{w}(\mathrm{~mm})$ & $b_{f}(\mathrm{~mm})$ \\
\hline $\mathrm{U} 1$ & & 2.00 & 80 & 70 \\
$\mathrm{U} 2$ & 800 & 2.00 & 90 & 70 \\
$\mathrm{U} 3$ & & 2.00 & 100 & 74 \\
\hline
\end{tabular}

Based on the specifications of the European standard EN 15512:2009 [17], the length considered for the uprights was $L=800 \mathrm{~mm}$. The upright and its perforations are shown in Fig. 4.
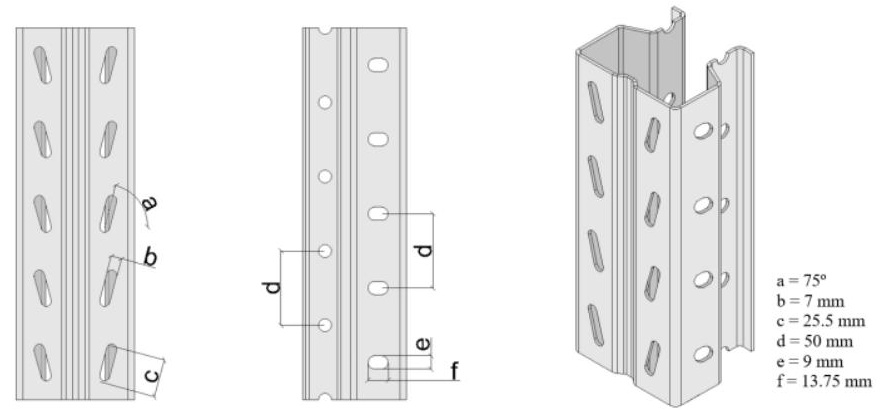

Fig. 4 Upright and perforations

Four rectangular hollow-section beams with different cross-sectional dimensions were considered, with different height $(h)$, width $(b)$, and thickness $(t)$, as presented in Fig. 5 and Table 2. Fig. 6 shows the cross-section properties of the studied uprights and beams.

The connection between the beams and the uprights is composed of five lips. The connection has the same dimension for all the prototypes tested. The lip dimensions are shown in Fig. 7. The prototypes tested were divided in three series, totalizing fifteen combinations of uprights and beams, which are listed in Table 3.

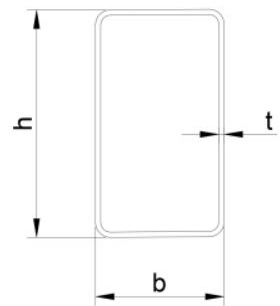

Fig. 5 Cross-section considered for beams

Table 2

Beam cross-section dimensions

\begin{tabular}{lcccc}
\hline Beam (type_t) & Length $(\mathrm{mm})$ & $b(\mathrm{~mm})$ & $h(\mathrm{~mm})$ & $t(\mathrm{~mm})$ \\
\hline B1_2.0 & 42 & 97 & 2.0 \\
B2_1.8 & 50 & 102 & 1.8 \\
\hline B2_2.0 & 50 & 102 & 2.0 \\
\hline B1 & 500 & 122 & \\
\hline
\end{tabular}

Fig. 6 Cross-section properties of the rack connection members (mm)

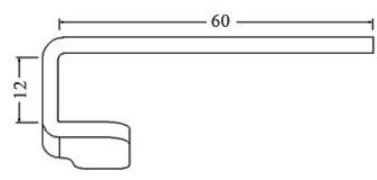

Fig. 7 Lip dimensions (mm)

Table 3

Series tested

\begin{tabular}{ccc}
\hline No. & Upright (type) & Beam (type_t $)$ \\
\hline \multirow{2}{*}{1} & $\mathrm{U} 1$ & B1_2.0 \\
& & B2_1.8 \\
& & B2_2.0 \\
2 & $\mathrm{U} 2$ & \\
& & B3_2.0 \\
\multirow{2}{*}{3} & $\mathrm{U} 3$ & B1_2.0 \\
& & B2_2.0 \\
& & B3_2.0 \\
\hline
\end{tabular}

\subsection{Material properties}

The upright and beam prototypes were fabricated from cold-formed steel. The values of yield stress $f_{y}$ and ultimate tensile strength $f_{u}$ were obtained from three tensile coupon tests, and the averaged results are presented in Table 4 . The tests reproduced the standard procedures for tensile testing presented in ABNT NBR 6892:2002 [18] 
Table 4

Tensile tests results.

\begin{tabular}{|c|c|c|c|}
\hline \multicolumn{2}{|c|}{ Prototype (type $\_t$ ) } & $f_{y}(\mathrm{MPa})$ & $\mathrm{fu}_{\mathrm{u}}(\mathrm{MPa})$ \\
\hline \multirow{3}{*}{ Upright } & U1_2.0 & 391 & 524 \\
\hline & U2_2.0 & 380 & 517 \\
\hline & U3_2.0 & 371 & 523 \\
\hline \multirow{4}{*}{ Beam } & B1_2.0 & 399 & 516 \\
\hline & B2_1.8 & 329 & 460 \\
\hline & B2_2.0 & 397 & 518 \\
\hline & B3_2.0 & 397 & 518 \\
\hline
\end{tabular}

\subsection{Test assembly, instrumentation, and data acquisition}

The tests were performed based on the specification of the European standard EN 15512:2009 [17]. The schematic diagram of the test set-up is depicted in Fig. 8. The upright's ends simulated a fixed-end condition and were fixed to a reaction frame that was fixed in the reaction slab. One beam was connected to one column in each test. The displacements on the upper and lower connector's face were obtained by displacement transducers (linear variable differential transformers (LVDT1 and LVDT2 at Fig. 8)).

Another displacement transducer (LVDT3 at Fig. 8) was positioned to obtain the vertical displacement under the loading applied by a jack. The measurements got by the displacement transducers (LVDTs) and the data were registered on an HBM Spider8 automatic data acquisition system. The ultimate moment capacity on the connections was calculated with the load applied by the jack and measured by the load cell. The connection stiffness was calculated based on the RMI [19] specifications. The cantilever type test set-up arrangement is shown in Fig. 9.

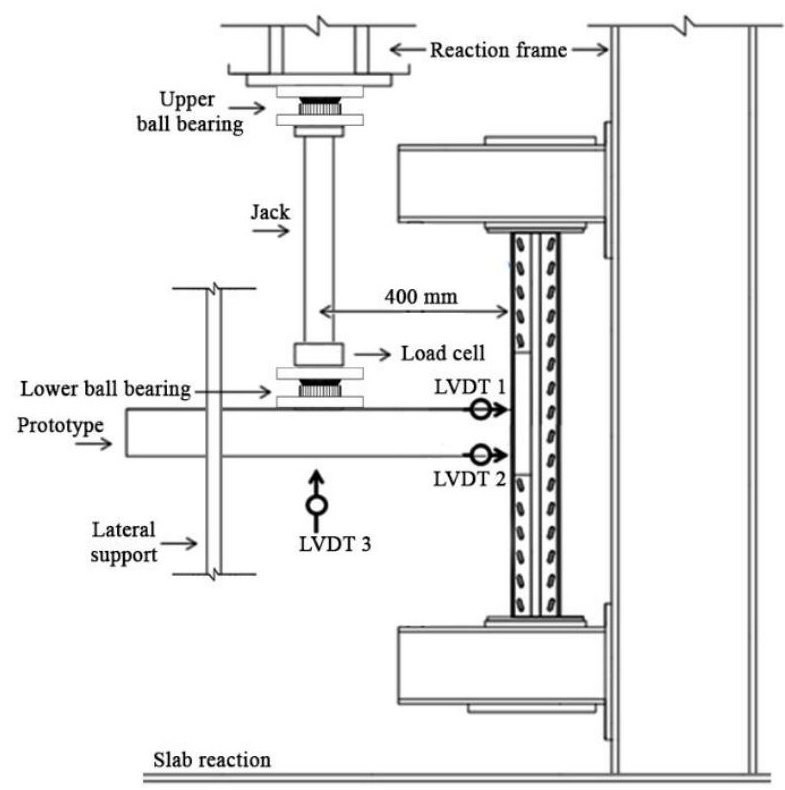

Fig. 8 Schematic diagram of the test set-up

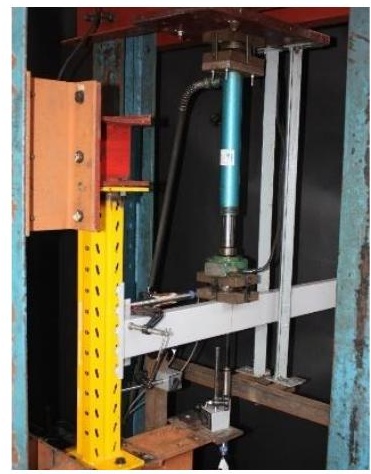

(a)

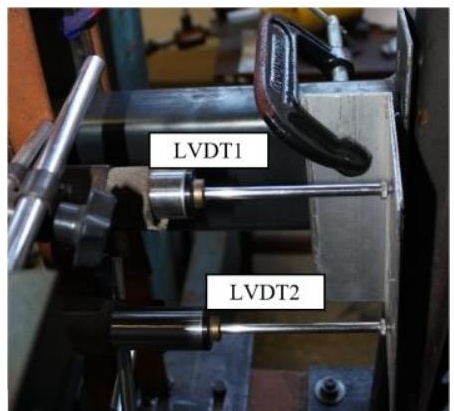

(b)

Fig. 9 Cantilever test set-up: (a) scheme and (b) position of LVDT1 and LVDT2

\subsection{Discussion on the test results}

The ultimate moment capacity on the connections was calculated by multiplying the load value applied by the jack and measured by the load cell by the distance of $400 \mathrm{~mm}$. The rotation $\theta$ at the ultimate moment was calculated using Eq. 1.

$\theta=\frac{\delta_{2}-\delta_{1}}{d}$

where:

$\delta_{1}, \delta_{2}$ are the deflections measured by LVDT1 and LVDT2;

$d$ is the distance between LVDT1 and LVDT2, and it equals the beam's height.

The averaged results of the failure load, ultimate moment capacity, and rotation at the ultimate moment in radians are shown in Table 5.

Table 5

Strength of the connector.

\begin{tabular}{cccc}
\hline Prototype & Failure load $(\mathrm{kN})$ & Ultimate moment & Rotation at the ultimate \\
\hline U1_2.0 $\times$ B1_2.0 & 4.28 & 1.71 & 0.064 \\
U1_2.0 $\times$ B2_1.8 & 4.24 & 1.71 & 0.057 \\
U1_2.0 $\times$ B2_2.0 & 4.34 & 1.74 & 0.059 \\
U2_2.0 $\times$ B3_2.0 & 5.43 & 2.17 & 0.051 \\
U3_2.0 $\times$ B1_2.0 & 4.64 & 1.86 & 0.056 \\
U3_2.0 $\times$ B2_2.0 & 4.47 & 1.79 & 0.061 \\
U3_2.0 $\times$ B3_2.0 & 5.41 & 2.06 & 0.049 \\
\hline
\end{tabular}

The initial stiffness of each experimental test and the respective average are shown in Table 7.

\section{Numerical Analysis}

A numerical study of 7 models was conducted to obtain reliable results of strength and stiffness for other arrangements of upright and beam cross sections. The ANSYS finite element software [16] was used to model the upright, beam, and connector with the five lipped connectors. The geometry, boundary, and loading conditions of the finite element model were developed to simulate the cantilever test, as shown in Fig. 10, with the same dimensions and details of the test prototypes and the material properties from the tensile tests results. The Young's modulus and Poisson's ratio considered for all members were $200 \mathrm{GPa}$ and 0.3 , respectively. The beam was connected to the upright using a connection with five lips (Fig. 11). 


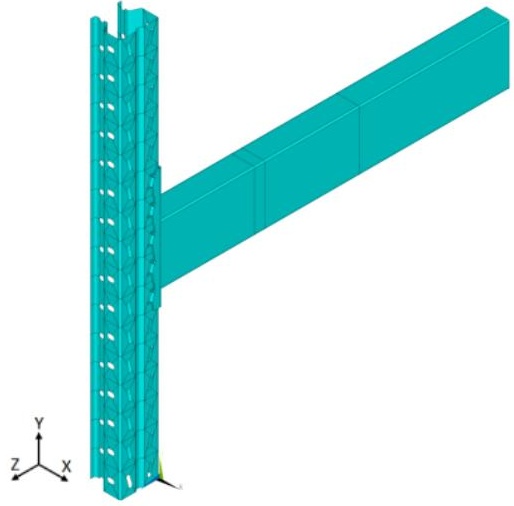

Fig. 10 Finite element model of the beam-upright connection

Geometric nonlinearity was considered adopting the full Newton Raphson solution method, and to simulate the physical nonlinearity, a multilinear model (multilinear isotropic hardening) with three points representing the stress-strain curve was adopted [20]. To solve the systems of linear equations, the sparse solver method was applied, which optimizes the process by reducing the number of operations required to solve the systems.

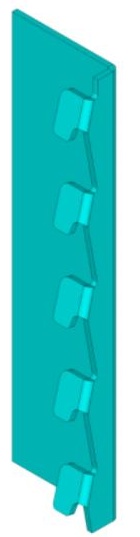

Fig. 11 Five lipped connectors

\subsection{Elements and mesh refinement}

The finite element used in the connector was SOLID45, which provided satisfactory results. It is an eight-node element with three degrees of freedom at each node, with plastic capacity. For the uprights and beams, the element used was SHELL181 [21], a four-node element with six degrees of freedom by node. To consider the contact between element, surface-to-surface contact elements [6], CONTA174 and TARGE170 were considered. Table 6 shows all the pairs used on the model.

\section{Table 6}

Tensile tests results.

\begin{tabular}{ccc}
\hline Contact & CONTA174 & TARGE170 \\
\hline Connector and upright & Upright's flange and web & Connector's plates \\
Lip and upright & Web perforation node lines & Surface of each lip \\
Beam and connector & Lateral beam nodes & Connector surface plate \\
\hline
\end{tabular}

Mesh sizes of 4, 5, and $6 \mathrm{~mm}$ were tested in the upright columns (Fig. 12a). The numerical results were compared to the experimental results and a better correlation was observed for the size of $5 \mathrm{~mm}$. In a similar way (Fig. 12b), the same study was performed on the beams for mesh sizes of 6,8 , and $10 \mathrm{~mm}$. In the beams, the mesh size was of $8 \mathrm{~mm}$. The mesh used in the analysis is presented in Fig. 13. On the interface of the solid-shell elements, a large stress concentration was observed, and $4 \mathrm{~mm}$ sides were used on the beam connector region to reduce this effect. Another study was conducted on the interface of the solid-shell elements regarding the number of layers in the solid element thickness, with 3, 4, and 5 layers. The results were very similar (Fig. 14). Therefore, the lowest value (3 layers) was used, because it presented a shorter processing time.

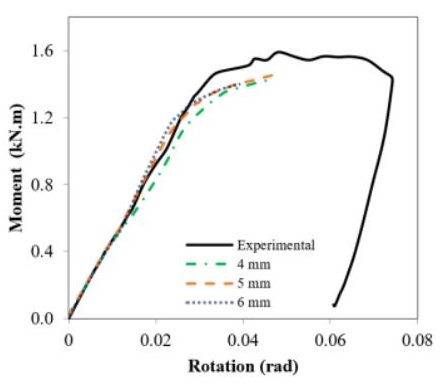

(a)

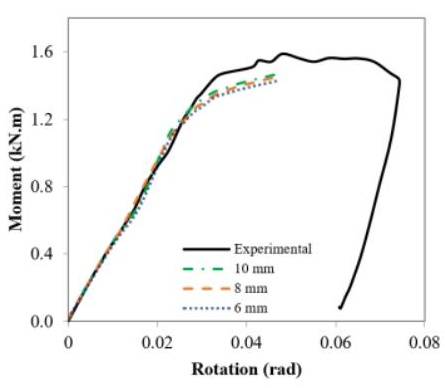

(b)
Fig. 12 Mesh study on prototype U1_2.0 × B1_2.0: (a) upright; (b) beam

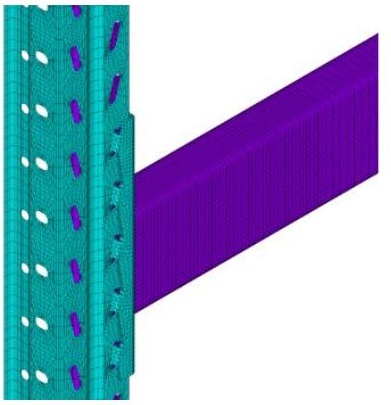

Fig. 13 Mesh used in ANSYS

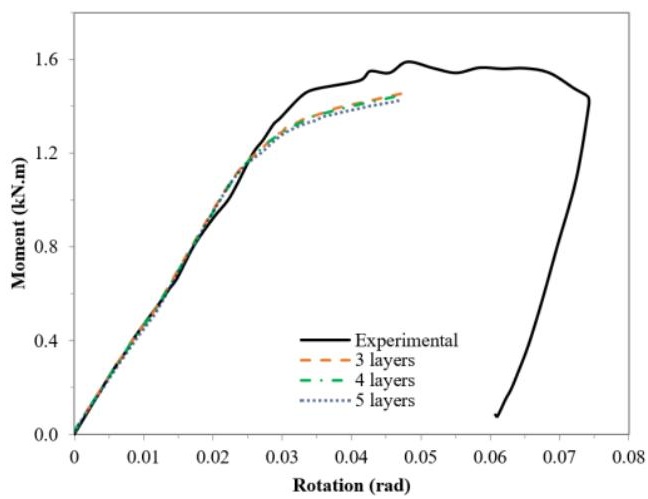

Fig. 14 Influence of number of layers on solid elements

\subsection{Boundary conditions}

The boundary conditions replicate the test assembly. The upper and lower upright end nodes had their rotations and translations restrained in all directions, simulating a fixed upright (see Fig. 15). The beams' lateral displacement was prevented by lateral displacement nodes stopped in the $\mathrm{x}$ direction (Fig. 16), which simulated the lateral support of the tests (Fig. 8). Their objective was to prevent lateral displacement of the beam.

The simulation of load application on the beam was performed incrementally, applying a displacement at the top of the beam (Fig. 16), in the position performed in the tests. The nodes of these lines were coupled, and a 20 mm-displacement was applied. An initial increment of $0.5 \mathrm{~mm}$ was defined, ranging from $0.1 \mathrm{~mm}$ to $1.0 \mathrm{~mm}$, according to the need evaluated automatically by Ansys.

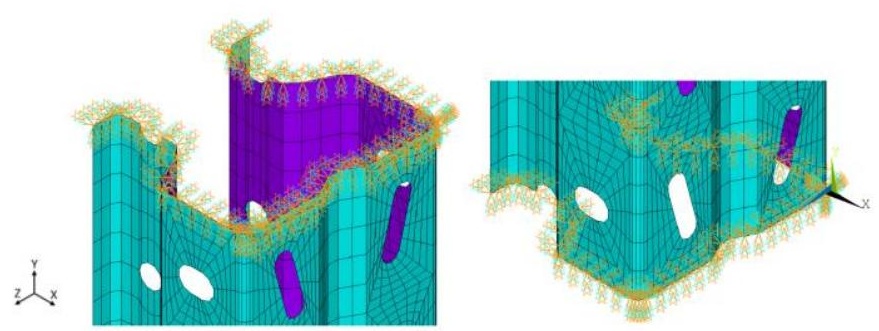

Fig. 15 Upright boundary conditions 


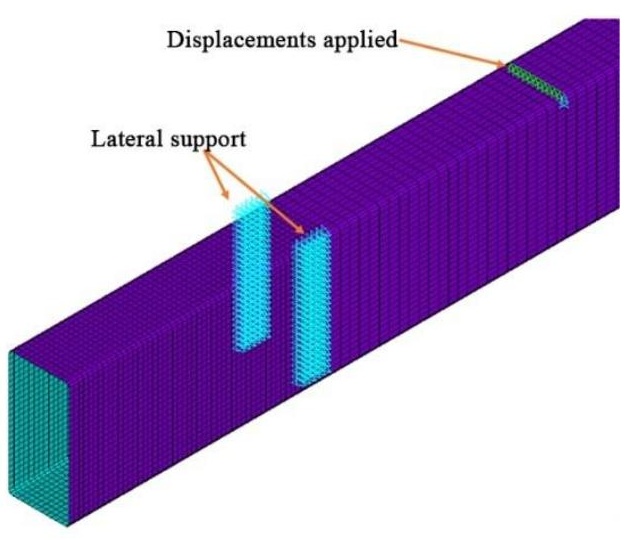

Fig. 16 Beam boundary conditions and load application

\subsection{Numerical results}

\subsubsection{Moment versus rotation curves}

The moment and rotation curves $(\mathrm{M}-\theta)$ of the numerical and experimental models were compared. In the numerical model, the displacement was gradually applied at the top of the beam, and the corresponding rotation, $\theta$, was calculated with Eq. 1 using the displacements of the $\mathrm{Z}$-axis of the nodes N1 and N2, as shown in Fig. 17.

The comparisons of the moment and rotation curves of the numerical and experimental models are presented in Fig. 18. The curves had a non-linear behavior. A satisfactory correlation was observed between the numerical and experimental curves. The important factors that influence this non-linearity are the slippage between the lipped connectors and the upright perforations, yielding of some regions of the lips due to a localized stress concentration effect, and geometrical imperfections in the tested prototypes. The observed differences in the experimental results of the same prototype were due to existent imperfections in the specimens.
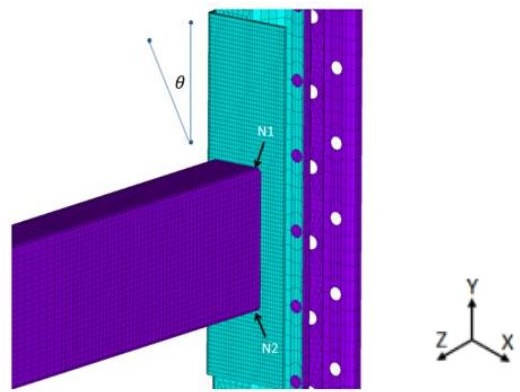

Fig. 17 Monitored nodes to measure rotation

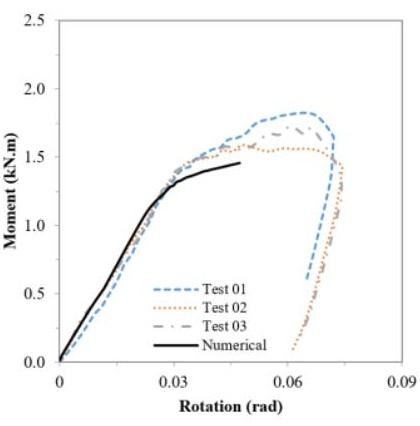

(a) U1_2.0 $\times$ B1_2.0

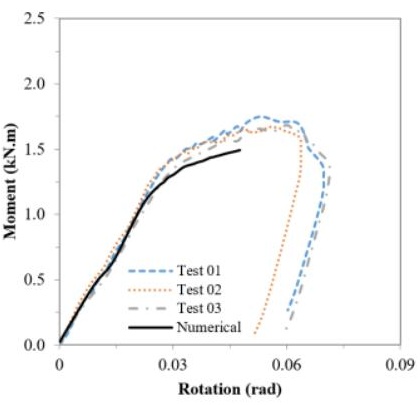

(b) $\mathrm{U} 1 \_2.0 \times$ B2__1.8

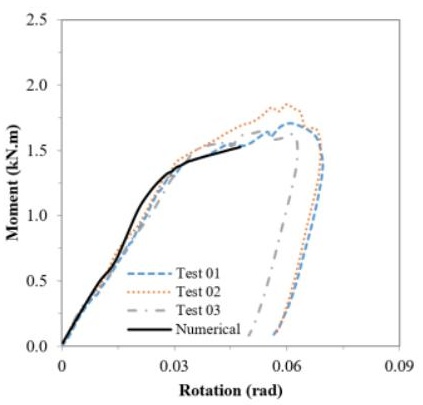

(c) U1_2.0 $\times$ B2_2.0

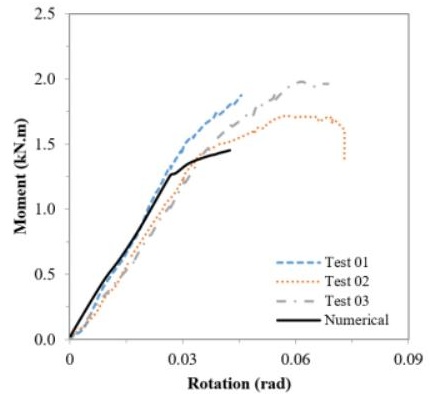

(e) $\mathrm{U}_{-} 2.0 \times$ B1_ 2.0

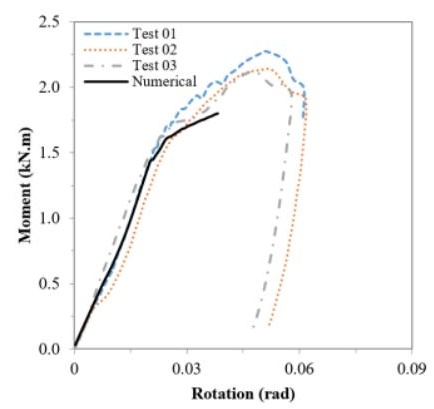

(d) $\mathrm{U} 2.2 .0 \times \mathrm{B} 32.0$

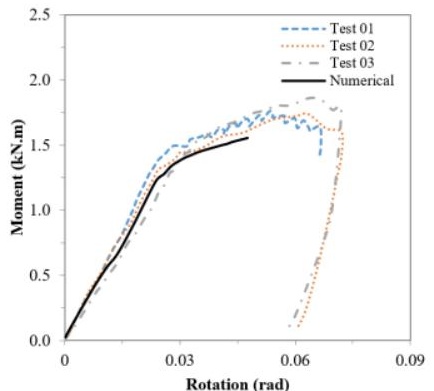

(f) $\mathrm{U} 3 \_2.0 \times$ B2_2.0

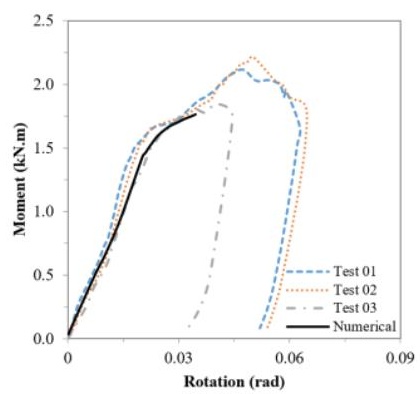

(g) $\mathrm{U}_{-} 2.0 \times \mathrm{B} 3{ }_{-} 2.0$

Fig. 18 Comparison of the numerical and experimental moment $\times$ rotation curves

\subsubsection{Comparisons of initial stiffnes}

The experimental and numerical initial stiffness of the models were compared. These values are presented in Table 7. The numerical ultimate moment capacity was always less than the experimental ultimate moment capacity. Therefore, the initial stiffness method was used to compute the connection stiffness. From the analysis of the results of stiffness of the experimental tests, it was concluded that some prototypes with the same dimensions presented significant differences in stiffness values. Again, there are significant influences of the imperfections in the results. The average stiffness of the numerical models had a good correlation with the average stiffness of the experimental analysis, presenting a maximum difference of $10 \%$.

Table 7

Comparison of experimental and numerical initial stiffness.

\begin{tabular}{ccccccc}
\hline & \multicolumn{3}{c}{ EXP } & \multicolumn{2}{c}{ NUM } & \\
\cline { 2 - 5 } Prototype & Test 1 & Test 2 & Test 3 & Average & Init. Stiff. & Dif (\%) \\
\hline U1_2.0 $\times$ B1_2.0 & 43.50 & 45.46 & 45.82 & 44.93 & 47.81 & 6.0 \\
U1_2.0 $\times$ B2_1.8 & 49.86 & 51.14 & 45.95 & 48.98 & 48.33 & 1.3 \\
U1_2.0 $\times$ B2_2.0 & 45.04 & 47.65 & 43.75 & 45.48 & 50.45 & 9.9 \\
U2_2.0 $\times$ B3_2.0 & 70.96 & 61.38 & 73.13 & 68.49 & 70.89 & 3.4 \\
U3_2.0 $\times$ B1_2.0 & 48.83 & 41.04 & 39.31 & 43.06 & 47.24 & 8.8 \\
U3_2.0 $\times$ B2_2.0 & 58.24 & 53.89 & 46.60 & 52.91 & 51.51 & 2.6 \\
U3_2.0 $\times$ B3_2.0 & 84.10 & 78.37 & 66.82 & 76.43 & 70.87 & 7.3 \\
\hline
\end{tabular}

After an analysis of the results, it was concluded that the moment versus rotation curves of the numerical model and experiments had similar behaviors, 
with the same formats in the linear section, and the stiffness of the numerical model was compatible with the stiffness of the experiments. Fig. 19 presents a schematic representation of the stiffness values of the numerical model and the experimental average stiffness. Considering the calculation of the initial stiffness, the results obtained with the proposed numerical model had values compatible with the experimental data. This indicates that the model can predict with good reliability the initial stiffness of the connection.

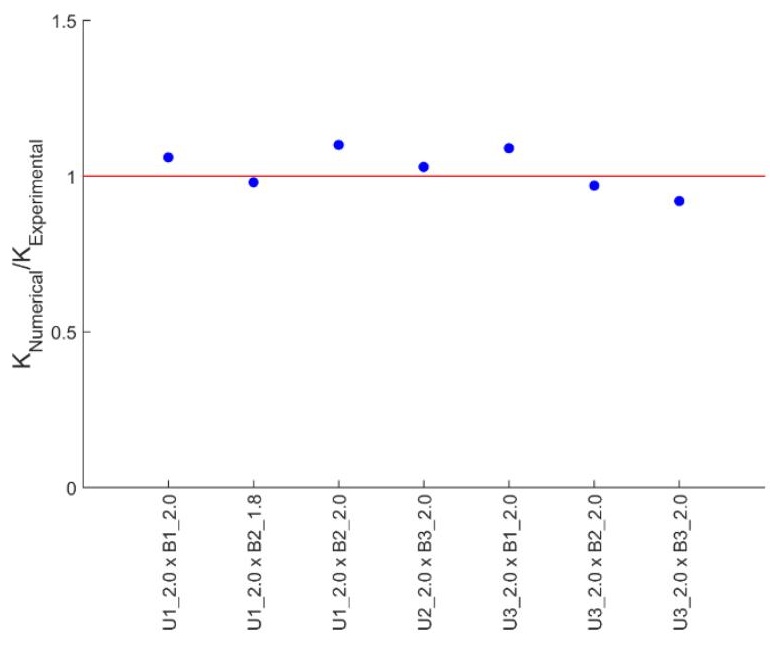

Fig. 19 Comparison of numerical and experimental stiffness

\section{Failure modes}

As observed in the analysis of the curves $(\mathrm{M}-\theta)$ of the experimental tests, there was a linear behavior from the beginning of the experiment. Imperfections and slack in the prototypes accentuated this behavior. In the numerical model, it was also possible to notice this behavior in the linear part of the curve. A study was carried out to identify the causes of this phenomenon in the substep of the numerical model where this behavior occurred.

The differences between the three types of beam (B1, B2, and B3) refer to the height and width of the cross section. Thus, the lips' solicitation will be different on each type. One model corresponding to each beam type was analyzed, as follows: (U3_2.0 $\times$ B1_2.0), (U3_2.0 × B2_2.0), and (U3_2.0 $\times$ B3_2.0).

It was identified that the displacements in the lipped connector had great influence on the changes in inclination in the initial part of the curve $(\mathrm{M}-\theta)$. Thus, these displacements were correlated with the points of the curve (M- $\theta)$ in which the model lost linearity. The displacement on the z-axis of the lipped connector was monitored, and it was identified as illustrated in Fig. 20.

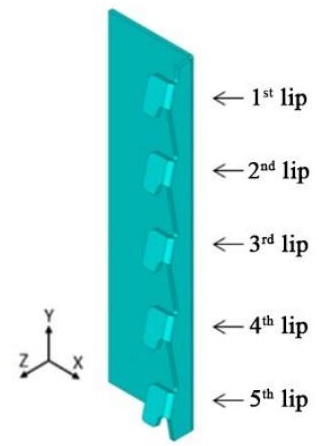

Fig. 20 Identification of lips and coordinate axes

The first analyzed model was U3_2.0 × B1_2.0. The M- $\theta$ curve of the experimental and numerical tests is shown in Fig. 21 . The vertical lines indicate points where the numerical model changes the slope of the curve. The first vertical line indicates a change in slope and a decrease in the rate of change in the $\mathrm{M}-\theta$ curve; the second one indicates another change in slope and an increase in the rate of change, and the third vertical line shows a decrease in the rate of change in $\mathrm{M}-\theta$ curve. The fourth vertical line indicates a sharp change in slope and loss of linearity of the numerical model. The displacement in each lip of the connector versus rotation is shown in Fig. 22. The vertical lines indicate points where the lips can change their behavior during the analysis.

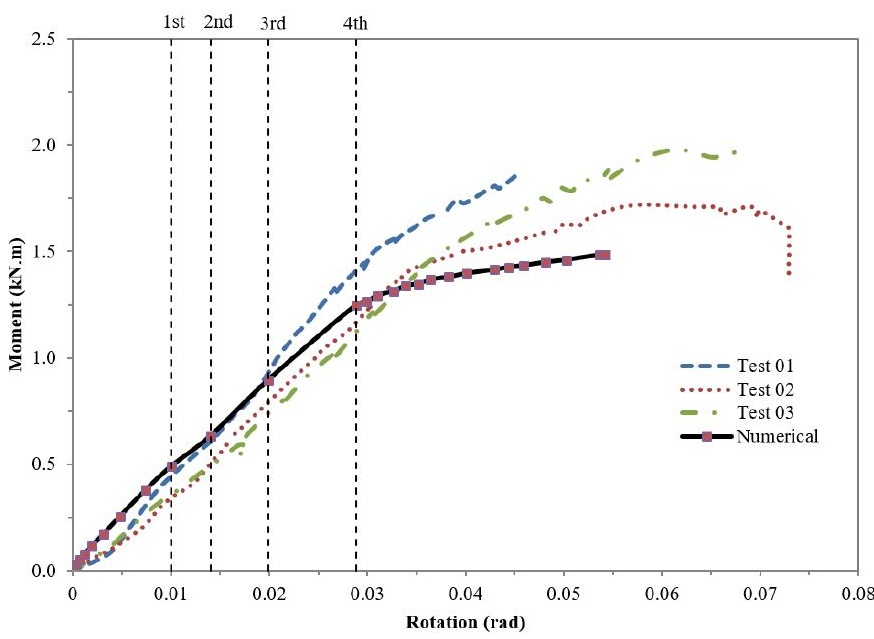

Fig. 21 Moment versus rotation of U3_2.0 $\times$ B1_2.0 model

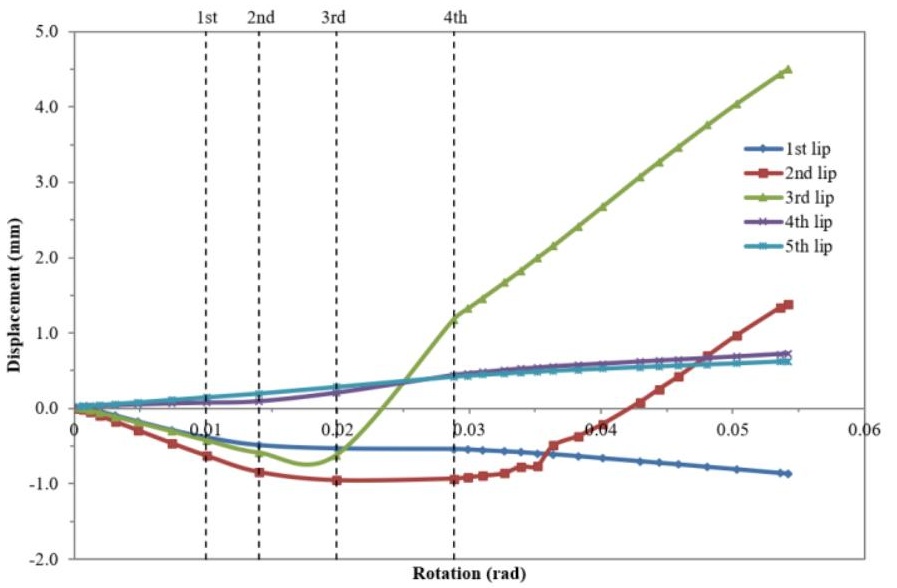

Fig. 22 Displacement versus rotation of each lip of U3_2.0 $\times$ B1_2.0 model

Note that the fourth and fifth lips do not contribute significantly in the resistance to rotation of the connection because their displacements are always positive in the z-axis, which is due to the compression force of the binary of the moment that appears in the base of the beam. Analyzing the von Mises stress in the numerical model in the substep of the second vertical line, the beginning of the yield at the base of the second and third lip is observed, and an increase in the slope of the curve $(\mathrm{M}-\theta)$ occurs owing to the absorption of the energy of the loading by these lips. It was observed a decrease in slope of the curve (M- $-\theta)$ from the third vertical line. This is because the third lip presents the entire section of its fold in a plastic state, no longer absorbing the energy of the load and only moving in the positive direction of the z-axis. The fourth vertical line marks the beginning of the loss of nonlinearity of the numerical model, coinciding with the beginning of the displacement in the positive direction of the z-axis of the second lip, because the section of its fold is already in a fully plastic state.

The collapse of the lipped connection occurred when there was an excessive deformation in the second lip, as can be seen in Fig. 23a. It is noted that the excessive displacement in the second lip did not occur in the numerical model (Fig. 23b), because it did not reach the rupture point.

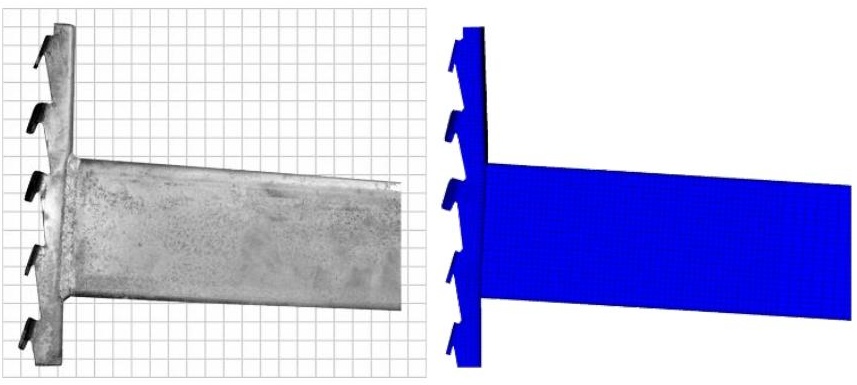

Fig. 23 Displacements on the lipped connection U3_2.0 × B1_2.0 model: (a) experimental; (b) numerical 
For the model U3_2.0 $\times$ B2_2.0, the moment versus rotation curve is shown in Fig. 24 and the displacement on each lip of the connector versus rotation is shown in Fig. 25. As in the previous analysis, the vertical lines helped to identify the points where the numerical model changed the slope of the curve and showed points where the lips changed their behavior during the analysis. As in the other model, the fourth and fifth lips did not contribute significantly to the resistance to rotation of the connection. In the substep corresponding to the third vertical line, the plastic deformation of the second and third lip begins. In the substep of the fourth vertical line, the section of the fold of the third lip is in a fully plastic state. Note that from the fifth vertical straight line, the model begins to lose linearity, coinciding with a change in the slope of the first lip, which can be characterized by the return of the slip. Small variations occur in the slope of the curve (M- $\theta$ ), until finally the section of the second lip fold is in a fully plastic state and starts to move in the positive $\mathrm{z}$-axis direction, causing the model to lose the non-linearity completely.

In none of the analyzed numerical prototypes, the model lost its linearity completely, and there was yield of the other elements that make up the connection, upright and beam, as can be seen in Fig. 26. Yield of the uprights did not occur during the entire analysis, and the beam had only a point of yield in the last loading step of the analysis.

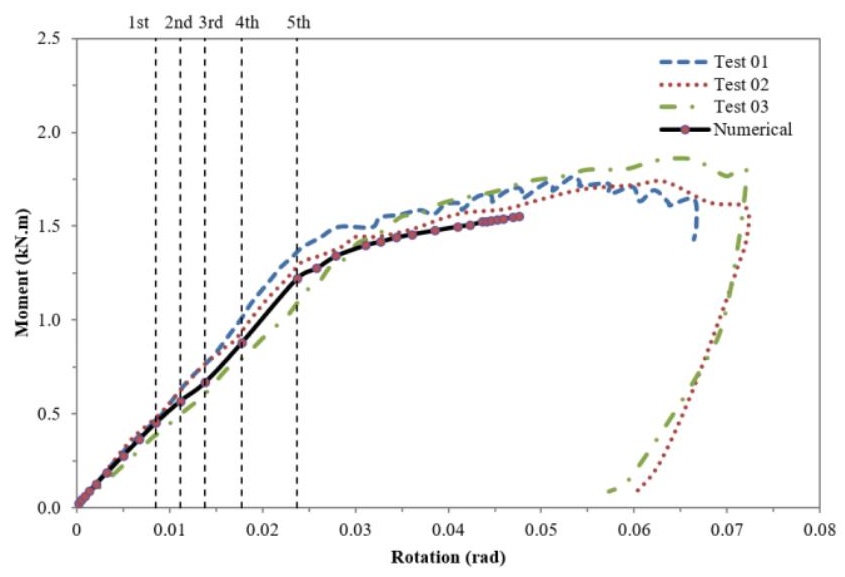

Fig. 24 Moment versus rotation of U3_2.0 $\times$ B2 _2.0 model

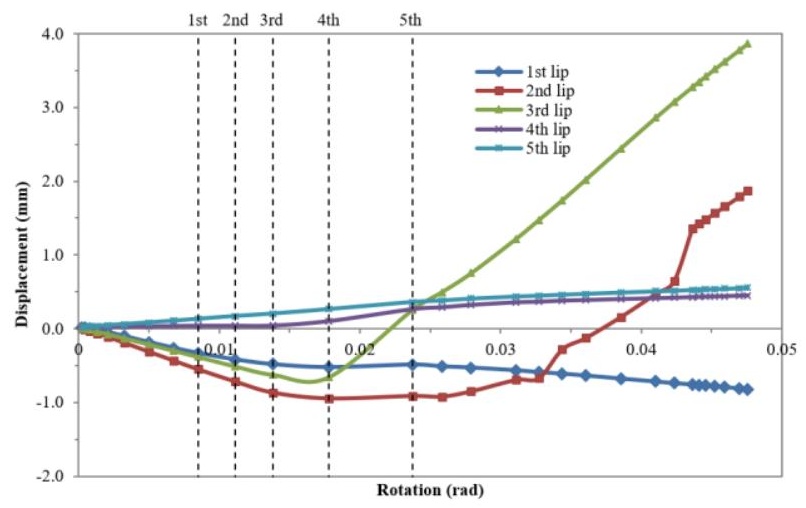

Fig. 25 Displacement versus rotation of each lip of U3_2.0 $\times$ B2_2.0 model

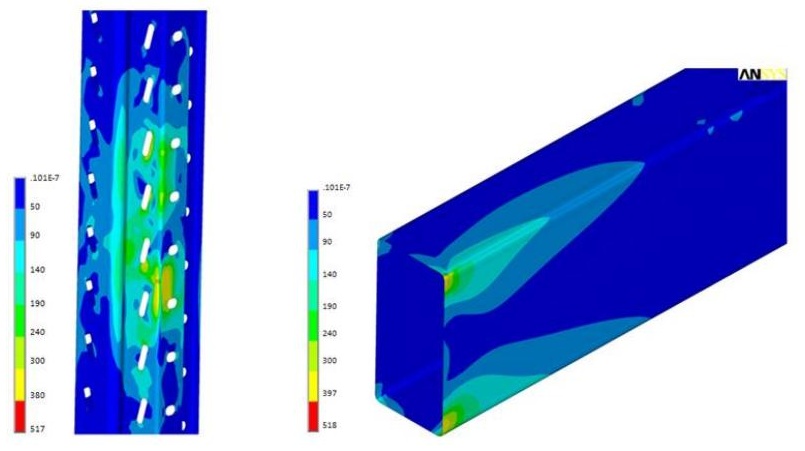

Fig. 26 von Mises stress of U3_2.0 × B2_2.0 model: (a) upright; (b) beam in Fig. 27, and the displacement on each lip versus rotation is shown in Fig. 28 A similar behavior is observed on the curves.

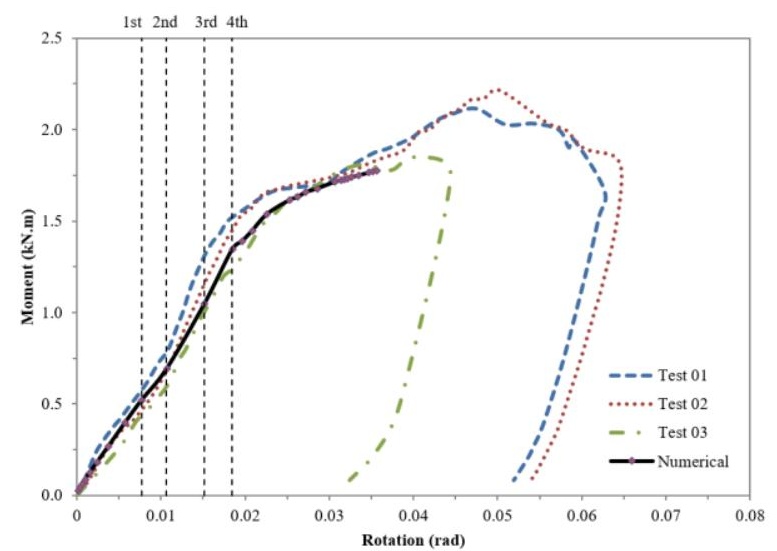

Fig. 27 Moment versus rotation of U3_2.0 × B3_2.0 model

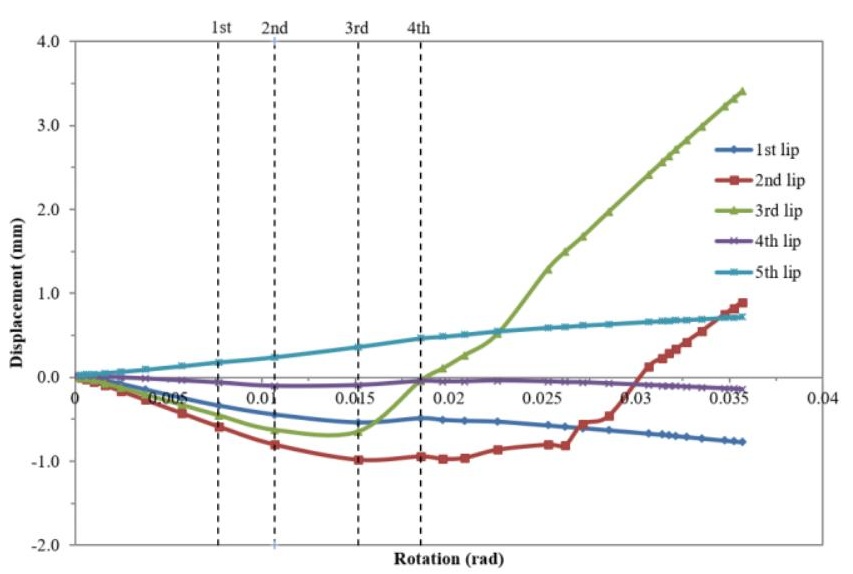

Fig. 28 Displacement versus rotation of each lip of U3_2.0 $\times$ B3_2.0 model

It was observed that, for this model, there was a greater contribution of the fourth lip in the resistance to rotation of the connection, whereas the fifth lip continued without a significant contribution. It can be noticed that the changes in the inclination of the curve M- $\theta$ are smoother. This is because the lips work together, without overloading any of them individually. At the starting point of the third vertical line (Fig. 28) the section of the third lip was already in a fully plastic state. As the first, second, and fourth lips are absorbing the energy of the loading, even with the beginning of the displacement of the third lip in the positive direction of the $\mathrm{z}$-axis, there is no change in slope of the curve M- $\theta$. From the fourth vertical line (Fig. 28) the second lip section is in an almost fully plastic state, but different from the other analyses, a contribution of the fifth lip in the resistance to rotation does not allow the second lip to be overloaded. The model continues with smooth changes in the slope of the curve M- $\theta$ until the entire section of the second lip is in a plastic state and begins its displacement in the positive direction of the $\mathrm{z}$-axis, thus resulting in a complete loss of linearity of the model.

The first and second models analyzed show similar lip behavior. The third model was different, with a greater contribution of the fourth lip in the resistance. This can be explained by the beam cross section. The first two have similar heights (97 and $102 \mathrm{~mm}$, respectively), and the last one is the highest (122 mm).

\section{Parametric analysis}

To better understand the influence of the geometric parameters on the connection stiffness in pallet rack structures, a parametric study was conducted with the models developed in the numerical analysis. The analysis was performed by changing the dimensions of the prototypes with commercial configurations. In total, 99 models were made with different combinations of beam and upright dimensions with the same connector's dimensions, and the stiffness values are depicted in Fig. 29. 


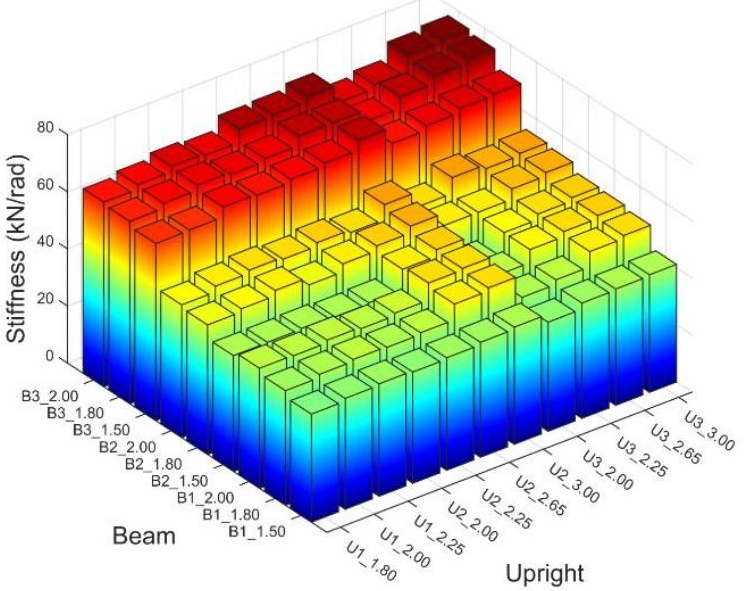

Fig. 29 Stiffness of parametric analysis

It was noted that by varying the dimensions of the beams while keeping the upright dimensions fixed, and then varying the beam dimensions and maintaining the upright dimensions fixed, a pattern of stiffness variation is observed. By varying the upright's web weight (grouped by types U1, U2, and U3) and maintaining its thickness fixed, the stiffness values of the models were compared. The largest variation observed was $4.24 \%$, when changing the size from U2 2.25 to U3 2.25. For this configuration, there is a $26 \%$ increase in moment of inertia in the axis of greater inertia.

By varying the thickness for the same upright type, it could be noticed that for type U1 and type U3, the greatest variation in stiffness occurred when there was a change in thickness from 2.0 to $2.25 \mathrm{~mm}$, presenting an increase of $2.68 \%$ and $6.34 \%$, respectively. For type U2, the greatest variation in stiffness occurred when the thickness changed from 2.25 to $2.65 \mathrm{~mm}$. In addition to the increase in moment of inertia, the increase in thickness of the upright generated a greater area of contact between the connector's lips and the holes of the upright.

By maintaining fixed the dimensions of the beam and changing its thickness, it was noticed that the greatest variations in stiffness occurred when the thickness changed from 1.5 to $1.8 \mathrm{~mm}$ for all types of beams, B1, B2, and B3, which presented an increase in stiffness of $5.78 \%, 9.32 \%$, and $1.70 \%$, respectively. With a variation in the cross-sectional dimensions of the beam (base and height), the greatest variation in stiffness occurred with the change from B2_1.5 to B3_1.5, generating an increase in stiffness of $32.91 \%$.

\section{Conclusions}

The objective of this work was the study of the connections in pallet storage systems fabricated in cold formed profiles, through experimental, numerical, and parametric analyses. The numerical model developed with the finite element method presented results with good correlation with those obtained by the experimental tests. For instance, the maximum difference between the numerical and experimental values of initial stiffness was $10 \%$. It was noticed that the behavior of the moment versus rotation curve of the experimental prototypes showed good correlation with the numerical values for the initial loading levels, with higher dispersion occurring from the beginning of the yield. Hence, the initial stiffness values were considered.

From the parametric analysis, a pattern was observed in the variation of stiffness when the dimensions of the prototypes were increased. The greatest variation was of $32.91 \%$, and resulted from increasing the height of the beam in $19.6 \%$.

The loss of linearity in the initial sections of the moment versus rotation curves, which occurred in the experimental tests, was caused by the variation in the displacements of the connector lips. It was noted that the third lip was the first to reach the yield stress and the first to generate displacement in the positive direction of the $\mathrm{z}$-axis. This displacement was observed in both the numerical and experimental analyses. The complete loss of non-linearity of the numerical model occurred when the section of the second lip fold was in a plastic state and began to move until the connection collapsed.

Finally, it was noticed that the upright and beam did not reach the yield stress at any point in the linear stretch of the moment versus rotation curve. To the point where the numerical model was able to reproduce the behavior of the connection, corresponding to a load that generated approximately $20 \mathrm{~mm}$ of displacement at the upper end of the beam, the upright did not reach the yield stress at any point. In other words, all the failure and collapse mechanisms occurred exclusively in the end connector.

\section{Acknowledgment}

The authors acknowledge the support provided by the National Council for Scientific and Technological Development (CNPq), Coordination of Improvement of Higher Level Personnel (CAPES), Minas Gerais State Research Support Foundation (FAPEMIG), Federal University of Ouro Preto (UFOP) and Águia Systems.

\section{References}

[1] Prabha P, Marimuthu V, Saravanan M, Arul Jayachandran S. Evaluation of connection flexibility in cold formed steel racks. J Constr Steel Res 2010;66:863-72. doi:10.1016/j.jcsr.2010.01.019.

[2] Shah SNR, Sulong NHR, Jumaat MZ, Shariati M. State-of-the-art review on the design and performance of steel pallet rack connections. Eng Fail Anal 2016;66:240-58 doi:10.1016/j.engfailanal.2016.04.017.

[3] Neiva LH de A, Sarmanho AMC, Faria VO, Souza FT de, Starlino JAB. Numerical and experimental analysis of perforated rack members under compression. Thin-Walled Struct 2018;130:176-93. doi:10.1016/j.tws.2018.05.024.

[4] Casafont M, Magdalena Pastor M, Roure F, Peköz T. An experimental investigation of distortional buckling of steel storage rack columns. Thin-Walled Struct 2011;49:933-46. doi:10.1016/j.tws.2011.03.016.

[5] Elias GC, Neiva LH de A, Sarmanho AMC, Alves VN, Castro AFB. Ultimate load of steel storage systems uprights. Eng Struct 2018;170:53-62. doi:10.1016/j.engstruct.2018.05.078.

[6] Bajoria KM, Talikoti RS. Determination of flexibility of beam-to-column connectors used in thin walled cold-formed steel pallet racking systems. Thin-Walled Struct 2006;44:372-80. doi:10.1016/j.tws.2006.01.007.

[7] Sena Cardoso F, Rasmussen KJR. Finite element (FE) modelling of storage rack frames. J Constr Steel Res 2016;126:1-14. doi:10.1016/j.jcsr.2016.06.015.

[8] Bajoria KM, Sangle KK, Talicotti RS. Modal analysis of cold-formed pallet rack structures with semi-rigid connections. J Constr Steel Res 2010;66:428-41. doi:10.1016/j.jcsr.2009.10.005.

[9] Markazi FD, Beale RG, Godley MHR. Experimental analysis of semi-rigid boltles connectors. Thin-Walled Struct 1997;28:57-87. doi:10.1016/s0263-8231(97)00003-7.

[10] Zhao X, Wang T, Chen Y, Sivakumaran KS. Flexural behavior of steel storage rack beamto-upright connections. J Constr Steel Res 2014;99:161-75. doi:10.1016/j.jcsr.2014.04.007.

[11] Aguirre C. Seismic behavior of rack structures. J Constr Steel Res 2005;61:607-24. doi:10.1016/j.jcsr.2004.10.001

[12] Escanio LA. Análise numérica de ligações em sistemas de armazenagem industrial. Master Dissertation, Ouro Preto: 2019

[13] Freitas AMS, Freitas MSR, Souza FT. Analysis of steel storage rack columns. J Constr Steel Res 2005;61:1135-46. doi:10.1016/j.jcsr.2005.02.004

[14] Freitas AMS, Freitas MSR, Souza FT, Silva GG, Faria VO. Theoretical and experimenta analysis of perforated rack columns. Rev Esc Minas 2013;66:289-94. doi:10.1590/S037044672013000300004

[15] Gusella F, Arwade SR, Orlando M, Peterman KD. Influence of mechanical and geometric uncertainty on rack connection structural response. J Constr Steel Res 2019;153:343-55. doi:10.1016/j.jcsr.2018.10.021

[16] ANSYS Inc. ANSYS. ANSYS, Inc 2014.

[17] EN 15512. Steel static storage system — Adjustable pallet racking systems - Principles for structural design 2009

[18] ABNT NBR ISO 6892:2002. Materiais metálicos - Ensaio de tração à temperatura ambiente. Assoc Bras Normas Técnicas 2002. doi:7704010.

[19] ANSI MH16.1. Specification for the Design, Testing and Utilization of Industrial Steel Storage Racks 2012

[20] Salmon, Charles; Johnson J. STEEL STRUCTURES: Design an Behavior. Emphasizing load and resistance factor design. 5th ed. 2008

[21] Freitas AMS, Souza FT, Freitas MSR. Analysis and behavior of steel storage drive-in racks Thin-Walled Struct 2010;48:110-7. doi:10.1016/j.tws.2009.09.003. 